\title{
Mikrostrukturelle Untersuchungen an Mangan-dotiertem Galliumnitrid mittels fortgeschrittener Methoden der hochauflösenden und analytischen Transmissionselektronenmikroskopie
}

\author{
Dissertation \\ zur Erlangung des Doktorgrades \\ der Mathematisch-Naturwissenschaftlichen Fakultäten \\ der Georg-August-Universität zu Göttingen
}

vorgelegt von

Tore Niermann

aus

Georgsmarienhütte

Göttingen 2006 
D 7

Referent: Herr PD Dr. M. Seibt

Korreferent: Herr Prof. Dr. R. Kirchheim

Tag der mündlichen Prüfung: 30.10.2006 


\section{Inhaltsverzeichnis}

1 Einleitung $\quad 7$

2 Einführung $\quad 11$

2.1 Galliumnitrid . . . . . . . . . . . . . . . . . . . 11

2.1 .1 Struktur . . . . . . . . . . . . . . . . . . . 11

2.1.2 Ausgedehnte Defekte in Wurtzit-GaN . . . . . . . . . . . . . . . 12

2.1.2.1 Stapelfehler . . . . . . . . . . . . . . . 13

2.1.2.2 Perfekte Versetzungen . . . . . . . . . . . . . . 15

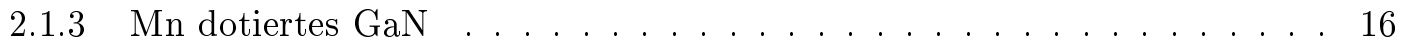

2.2 Moderne Transmissionselektronenmikroskopie . . . . . . . . . . . . . . . . 18

2.2.1 Abbildende Transmissionselektronenmikroskopie . . . . . . . . . . . . . 19

2.2.2 Analytische Transmissionselektronenmikroskopie . . . . . . . . . . . . . 20

3 Hochaufgelöste Transmissionselektronenmikroskopie 23

3.1 Abbildung durch das Elektronenmikroskop . . . . . . . . . . . . . . . . 23

3.1 .1 Linsenfehler . . . . . . . . . . . . . . . . . . . . 25

3.1 .2 Bildentstehung . . . . . . . . . . . . . . . . . . 28

3.1.2.1 Allgemeine Bildentstehung . . . . . . . . . . . . . 28

3.1.2.2 Quasikohärente Näherung . . . . . . . . . . . . . . . . . . 30

3.1.2.3 Lineare Abbildung . . . . . . . . . . . . . . . . . . 31

3.1.3 Einfluss der CCD-Kamera . . . . . . . . . . . . . . . . . . . 32

3.2 Prinzip der Defokusserienrekonstruktion . . . . . . . . . . . . . 32

3.3 Streuung im Objekt . . . . . . . . . . . . . . . . . 34

3.3.1 Starkes und schwaches Phasenobjekt . . . . . . . . . . . 35

3.3 .2 Elastische kohärente Streuung . . . . . . . . . . . . . . . . . . . 36

3.3 .3 Thermisch diffuse Streuung . . . . . . . . . . . . . . . . . 36

3.3.4 Inelastische Streuung . . . . . . . . . . . . . . . . . . . . . . 37

3.3.5 Stobbs-Faktor . . . . . . . . . . . . . . . . . . . . . 37

3.4 Bestimmung der Linsenfehler . . . . . . . . . . . . . . . . . . . . 38

3.4.1 Symmetrische Aberrationen . . . . . . . . . . . . . . . . . 38

3.4 .2 Antisymmetrische Aberrationen . . . . . . . . . . . . . . . . 38

4 Objektwellenrekonstruktion $\quad 41$

4.1 Experimentelles Vorgehen . . . . . . . . . . . . . . . . . . . . 42

4.2 Messung der Bildverschiebung . . . . . . . . . . . . . . . . . . . . 43

4.3 Messung der Linsenfehler . . . . . . . . . . . . . . . . . . . . . . . . 44

4.3.1 Abschätzung der benötigten Genauigkeit . . . . . . . . . . . . . . 46

4.3.2 Bestimmung von Defokus und Astigmatismus . . . . . . . . . . . . . . . . . 47

4.3.2.1 Vorbetrachtung . . . . . . . . . . . . . . . . . . . 49 
4.3.2.2 Vorverarbeitung der Diffraktogramme . . . . . . . . . . . . 49

4.3.2.3 Abschätzung des Hintergrundes $B(q)$ und der Einhüllenden $\mathcal{E}(q) \quad 50$

4.3.2.4 Anpassung des Defokus und des Astigmatismus . . . . . . . . . 51

4.3.2.5 Abschätzung der erreichten Genauigkeit . . . . . . . . . . . 51

4.3.3 Berechnung der Aberrationskonstanten . . . . . . . . . . . . . 53

4.4 Vergleich zwischen rekonstruierter und simulierter Wellenfunktion . . . . . . . 55

4.5 Kontrollmessung . . . . . . . . . . . . . . . . . . . . 56

5 Ausgedehnte Defekte in GaN $\quad 61$

5.1 Geometric Phase Analysis . . . . . . . . . . . . . . . . . . . 6 61

5.1 .1 Grundidee . . . . . . . . . . . . . . . . . . 6 61

5.1 .2 Messung der Verschiebungsfelder . . . . . . . . . . . . . . . 62

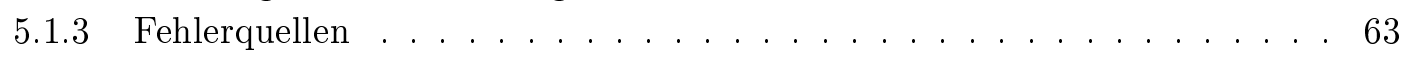

5.2 Wachstum und Geometrie der Proben . . . . . . . . . . . . . . . 63

5.3 Basale Stapelfehler in GaN:Mn . . . . . . . . . . . . . . . . . . . 65

5.4 Aufgespaltene $60^{\circ}$-Versetzung . . . . . . . . . . . . . . . 65

5.5 Kleinwinkelkorngrenzen . . . . . . . . . . . . . . . . 73

5.6 Simulationen von prismatischen Stapelfehlern . . . . . . . . . . . . . 73

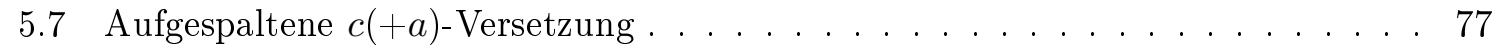

5.8 Weitere Partialversetzungen . . . . . . . . . . . . . . . . 81

5.8 .1 Spiegelvarianten . . . . . . . . . . . . . . . . . . . . . . 81

5.8 .2 Stairrod-Versetzung . . . . . . . . . . . . . . . . 81

6 Mn-Einbau in GaN $\quad 87$

6.1 Ausscheidungen . . . . . . . . . . . . . . . . . . 87

6.2 Gelöstes Mn . . . . . . . . . . . . . . . . . . . . . . 93

7 Gitterplatzbestimmung $\quad 105$

7.1 Channeling und anomale Absorption . . . . . . . . . . . . . . . 105

7.1.1 Grundidee von ALCHEMI . . . . . . . . . . . . . . . . . . . . . . . . . . . . . . . . . . . . . .

7.1 .2 Delokalisierung . . . . . . . . . . . . . . . . . . . 108

7.2 Vorgehensweise bei GaN:Mn . . . . . . . . . . . . . . . . . . . . . 109

7.3 Experimentelles Vorgehen . . . . . . . . . . . . . . . . . . . 110

7.4 Simulationen . . . . . . . . . . . . . . . . . . . . . . . 112

7.5 Anpassung und Vergleich der Profile . . . . . . . . . . . . . . . . . . . 113

7.6 Testmessung an $\mathrm{Al}_{0.23} \mathrm{Ga}_{0.77} \mathrm{~N} \ldots \ldots \ldots \ldots \ldots \ldots \ldots$

7.7 Gitterplatz des $\mathrm{Mn}$ in $\mathrm{GaN} \ldots \ldots \ldots \ldots \ldots$

8 Zusammenfassende Diskussion und Ausblick 123

$\begin{array}{lr}\text { A Kalibrierung der Strahlverkippung } & 127\end{array}$

A.1 Strahlverkippung im Beugungsbild . . . . . . . . . . . . . . . . 127

A.2 Umrechnung Beugungsbild-Diffraktogramm . . . . . . . . . . . . . . . . . . 129

A.3 Aufhebung der Doppeldeutigkeit . . . . . . . . . . . . . . . . . . . . . 130

A.4 Kalibrierung . . . . . . . . . . . . . . . . . . . . . . . . . 130 
Inhaltsverzeichnis

$\begin{array}{ll}\text { B Anisotrope Elastizitätstheorie von Versetzungen } & 133\end{array}$

B.1 Verschiebungsfeld . . . . . . . . . . . . . . . . . . . . . . . . 134

B.2 Stapelfehlerenergie . . . . . . . . . . . . . . . . . 135

$\begin{array}{ll}\text { C Phase an der Ausscheidungsoberfläche } & 137\end{array}$

$\begin{array}{ll}\text { D Probenpräparation } & 141\end{array}$

D.1 Querschnittsproben . . . . . . . . . . . . . . . . . . . . 141

D.2 Aufsichtproben . . . . . . . . . . . . . . . . . . . 141

$\begin{array}{ll}\text { E Parameter des Mikroskops } & 143\end{array}$

$\begin{array}{ll}\text { Literaturverzeichnis } & 144\end{array}$ 


\section{Einleitung}

Die Leistungssteigerung von Halbleiterbauelementen wurde in den letzten Jahrzehnten im Wesentlichen durch eine Verkleinerung der Strukturgrößen erreicht. Bei weiterer Miniaturisierung werden in den nächsten Jahrzehnten Größen erreicht, bei denen die Bauelemente von Quanteneffekten dominiert werden. So wird beispielsweise die Austauschwechselwirkung zwischen den Spins der Ladungsträger nicht mehr zu vernachlässigen sein. Die Spintronik ist ein Gebiet mit dem neuen Konzept, neben der Ladung gerade auch den Spinfreiheitsgrad der Elektronen als Informationsträger zu verwenden und schon so explizit quantenmechanische Eigenschaften auszunutzen.

Gegenüber herkömmlichen Bauelementen sind für Spintronik-Anwendungen größere Datenverarbeitungsgeschwindigkeiten und auch ein geringerer elektrischer Leistungsbedarf zu erwarten [1]. Weitere Vorteile gegenüber der herkömmlichen Elektronik ergeben sich, wenn quantenmechanische Effekte ausgenutzt werden sollen: so unterscheiden sich z.B. die Dephasierungszeiten, d.h. die Lebenszeiten präparierter Zustände, von Spinanregungen $(\approx 100 \mathrm{~ns}$ in GaAs bei $5 \mathrm{~K}$ [2]) um Größenordnungen von denen von Elektronen-Ortswellenfunktionen $(<10 \mathrm{ps}$ in GaAs bei $5 \mathrm{~K}$ [3]). Mit der Kontrolle des Spinzustands ergibt sich auch eine mögliche Realisierung von Quanten-Bit Operationen, die z.B. für einen Quanten-Computer nötig wären [4].

Eine ideale Kombination der Spintronik mit herkömmlicher Elektronik ließe sich in HalbleiterSystemen realisieren. Ein wichtiger Punkt für die Halbleiter-Spintronik ist dabei die effiziente Injektion spinpolarisierter Elektronen in den Halbleiter. Mit einer direkten Injektion aus ferromagnetischen metallischen Kontakten lässt sich allerdings aufgrund der großen Unterschiede in der Leitfähigkeit der Materialien nur ein sehr geringer Polarisationsgrad erreichen [5]. Eine Polarisation ist höchstens bei der Injektion über hochohmische Kontakte, wie Schottky-Barrieren oder Tunnelkontakte [6], zu erreichen. Ein weiterer Nachteil von metallischen Kontakten ist, dass der Abstand zu den funktionalen Halbleiterbereichen zu groß werden könnte, so dass die Spinrelaxation die Polarisierung während des Transportes zerstört.

Ferromagnetische Halbleiter als Injektoren weisen gegenüber metallischen Injektoren derartige Nachteile nicht auf, da weder große Leitfähigkeitsunterschiede existieren, noch große Transportwege nötig werden. Ferromagnetismus in Halbleitern lässt sich auf zwei Weisen erreichen. Zum einen gibt es intrinsische magnetische Halbleiter, wie z.B. Europium-Chalkogenide. Allerdings unterscheiden sich die Kristallstrukturen dieser Halbleiter meistens von denen konventioneller Halbleiter, wie Si, GaAs oder GaN, und ihre Herstellung ist sehr schwierig. Die andere Möglichkeit sind verdünnte magnetische Halbleiter („Diluted Magnetic Semiconductors“; DMS). Hier werden herkömmliche Halbleiter mit magnetischen Ionen bzw. Atomen dotiert, um eine magnetische Ordnung der Ladungsträger zu erreichen. Ohno et al. zeigten bereits 1998 für Mn-dotiertes GaAs, dass verdünnte magnetische Halbleiter mit ferromagnetischen Eigenschaften (Curie-Temperatur: $110 \mathrm{~K}$ ) möglich sind [7].

Eine Vorraussetzung für technische Spintronik-Anwendungen wären DMS-Materialien mit einer Curie-Temperatur oberhalb der Raumtemperatur. Theoretische Arbeiten sagen diese für 
Mn-dotiertes GaN hervor [8, 9], was viele Forschungstätigkeiten an diesem System ausgelöst hat $[10,11]$. In der Literatur herrscht heutzutage ein uneinheitliches Bild, was die experimentell beobachteten magnetischen Eigenschaften von GaN:Mn angeht. Auch Aussagen von theoretischer Seite zu den verantwortlichen magnetischen Mechanismen sind uneinheitlich.

Ein Grund für die uneinheitlichen experimentellen Befunde der magnetischen Eigenschaften könnte die unterschiedliche Mikrostruktur der untersuchten Proben sein. Daher ist eine sorgfältige Charakterisierung der Proben nötig. Die GaN-Schichten werden im Allgemeinen mittels Molekularstahlepitaxie (,Molecular Beam Epitaxy“; MBE), wie die in dieser Arbeit untersuchten Proben, oder durch metallorganische chemische Gasabscheidung ("Metal-Organic Chemical Vapour Deposition“; MOCVD) hergestellt. Eine genaue Charakterisierung der Proben ermöglicht auch, das Wachstum der Proben zu optimieren. Da die Löslichkeit von Mn in $\mathrm{GaN}$ sehr gering ist, lag bei den anfänglichen Untersuchungen das Hauptinteresse darin, Wachstumsbedingungen zu finden, bei denen Mn in GaN eingebaut wird. Der nächste Schritt ist eine Optimierung des Wachstums, um die gewünschten Eigenschaften der Schicht, wie z.B. Schichtqualität, Konzentration des gelösten Mn und Unterdrückung von Ausscheidungen durch Variation der Wachstumsbedingungen zu verbessern.

Diese Arbeit zeigt, wie die Transmissionselektronenmikroskopie (TEM) für diese Charakterisierung genutzt werden kann. Die analytische Elektronenmikroskopie kann bei der Untersuchung des Mn-Einbaus weitere Erkenntnisse erbringen. So wird beispielsweise die Identifizierung der in dieser Arbeit beobachteten $\mathrm{GaMn}_{3} \mathrm{~N}$-Ausscheidungen erst durch eine Kombination von abbildenden und analytischen Methoden möglich. Auch eine ungleichmäßige Verteilung des gelösten Mn lässt sich so beobachten, wie später gezeigt wird. Ein wichtiger Punkt für die magnetischen Eigenschaften ist der substitutionelle Einbau des Mn. Über winkelaufgelöste Channelingexperimente bietet das TEM auch die Möglichkeit zu quantifizieren, zu welchen Anteilen das Mn substitutionell eingebaut wird.

Die erreichbare direkt interpretierbare Aufösung, d.h. die Punktaufösung, in der konventionellen hochaufösenden TEM („High Resolution TEM“; HRTEM) wird für Mittelspannungsgeräte im Wesentlichen durch die starken Linsenfehler, insbesondere durch die sphärische Aberration, begrenzt. Zwar lassen sich mit diesen Geräten auch noch kleinere räumliche Informationen abbilden, allerdings sind diese Informationen nur noch aufwendig durch Vergleich mit Bildsimulationen interpretierbar.

Neue holographische Ansätze wie z.B. die Objektwellenrekonstruktion ermöglichen es, diese Einschränkung zu überwinden, indem direkt die Elektronenwelle untersucht wird, wie sie auf der Rückseite der Probe zu finden ist. Da der nachteilige Effekt der Linsenfehler hier korrigiert werden kann, haben diese Rekonstruktionen eine Punktauflösung, die nur noch durch die Stabilität des Mikroskops begrenzt ist.

Mit der Objektwellenrekonstruktion wird es so möglich, eine Vielzahl ausgedehnter Kristalldefekte hochaufösend abzubilden und direkt zu interpretieren, und das auch unter für konventionelle HRTEM problematischen Bedingungen. So können auch schräg in der Probe liegende Versetzungen analysiert oder Reaktionen zwischen mehreren Versetzungen und Stapelfehlern beobachtet werden.

Messungen der Verzerrungsfelder dieser Defekte sind mit konventioneller HRTEM nur sehr eingeschränkt und unter großen Aufwand möglich [12]. Diese Messung ist gerade in Kristallstrukturen ohne Inversionssymmetrie, wie z.B. GaN, aufgrund der erhöhten Sensitivität der 
konventionellen Abbildung auf Änderung der Probendicke und -orientierung schwierig. Durch die Objektwellenrekontruktion ergeben sich praktische Möglichkeiten die Verzerrungsfelder dieser Defekte ohne weitere Einschränkungen zu messen, wie in dieser Arbeit gezeigt wird.

Die vorliegende Arbeit gliedert sich folgendermaßen: Kapitel 2 vermittelt einen Überblick über ausgedehnte Defekte in GaN, über den Forschungsstand im DMS-System GaN:Mn und über aktuelle Entwicklungen der abbildenden und analytischen Transmissionselektronenmikroskopie. Das Kapitel 3 gibt eine ausführlichere Einführung in die hochauflösende TEM im Hinblick auf die Objektwellenrekonstruktion. Diese Methode wird in Kapitel 4 ausführlicher vorgestellt. Ihre Anwendung zur Untersuchung von ausgedehnten Defekten in GaN wird in Kapitel 5 gezeigt. Dabei wird es möglich, verschiedene Arten von Stapelfehlern zu unterscheiden sowie die Kernstrukturen der Partialversetzungen und Reaktionen von Partialversetzungen mit Stapelfehlern direkt zu beobachten. Auch die Verzerrungsfelder der Defekte lassen sich messen. In Kapitel 6 wird der Einbau des Mn in die GaN-Schicht und sein Einfluss auf die Schichtmorphologie untersucht. Dabei wurden $\mathrm{GaMn}_{3} \mathrm{~N}$-Ausscheidungen und eine inhomogene Verteilung des Mangans beobachtet. Eine Methode, das Verhältnis zwischen substitutionell und interstitiell eingebauten Mn zu messen, wird in Kapitel 7 vorgestellt. Mit dieser Methode kann nachgewiesen werden, dass das Mn zu 96\% substitutionell in das GaN-Gitter eingebaut wird. Im abschließenden Kapitel 8 werden die Ergebnisse zusammengefasst und diskutiert, sowie ein Ausblick auf mögliche weitere Entwicklungen gegeben. 


\section{Einführung}

\subsection{Galliumnitrid}

Halbleiter auf GaN-Basis haben einen direkten Bandübergang mit einer Bandlücke von $3.4 \mathrm{eV}$ bei Raumtemperatur. Dieser Bandübergang macht GaN zu einem idealen Materialsystem für optoelektronische Anwendungen.

Aufgrund des hohen Schmelzpunktes von GaN, der bei etwa $2800{ }^{\circ} \mathrm{C}$ liegt, und des hohen Dampfdrucks des Stickstoffs bei diesen Temperaturen von über 45 kbar ist es nur in Speziallaboren möglich, GaN-Kristalle aus der Schmelze herzustellen. Daher werden GaN-Strukturen durch Heteroepitaxie auf Substraten wie Saphir, SiC oder Si mittels Molekularstahlepitaxie („Molecular Beam Epitaxy“; MBE) oder metallorganischer chemischer Gasabscheidung ("Metal-Organic Chemical Vapour Deposition"; MOCVD) hergestellt. Die großen Unterschiede der Gitterkonstanten von $-16 \%$ bis $+20 \%$ und die großen Unterschiede in den thermischen Ausdehnungskoeffizienten von $-54 \%$ bis $+34 \%$ zwischen dem GaN und dem Substrat führen beim Schichtwachstum bzw. beim Abkühlen der Proben von der Wachstumstemperatur $\left(600^{\circ}\right.$ bis $1000^{\circ}$ C) zum Einbau vieler ausgedehnter Kristalldefekte. Die Anzahl dieser Defekte kann durch bestimmte Wachstumstechniken wie z.B. dem Einsatz von Pufferschichten verringert werden.

Die Kristallstruktur des GaN wird im Abschnitt 2.1.1 vorgestellt. Einen Überblick über ausgedehnte Defekte in wurtzitischem GaN gibt Abschnitt 2.1.2.

Theoretische Arbeiten prognostizierten im Jahr 2000 Ferromagnetismus bei Raumtemperatur für 5 at\% Mn-dotiertes GaN [8]. Dies machte GaN:Mn zu einem hoffnungsvollen Kandidaten für einen verdünnten magnetischen Halbleiter, und damit zu einem möglichen Material für Spintronik-Anwendungen. Abschnitt 2.1.3 geht auf diese Thematik weiter ein.

Eine ausführlichere Einführung in das Materialsystem GaN wird z.B. im Artikel von Weber et al. [13] gegeben.

\subsubsection{Struktur}

GaN tritt in zwei verschiedenen Polytypen auf: in der Wurtzit-Struktur $(2 \mathrm{H})$ oder in der Zinkblende-Struktur (3C). Die Wurtzit-Struktur ( $\alpha$-Phase) ist die thermodynamisch stabile Phase. Sie besteht aus zwei hexagonal dichtest gepackten Untergittern mit den Gitterkonstanten $a=0.3190 \mathrm{~nm}$ und $c=0.5189 \mathrm{~nm}$, die um $0.377 \cdot[0001]$ gegeneinander verschoben sind [14].

Das hexagonale Gitter ist in Abb. 2.1 illustriert. Die Grundebene wird basale Ebene genannt und hat die Miller-Indizierung (0001). Ebenen, die senkrecht auf der Basalebene stehen, also 


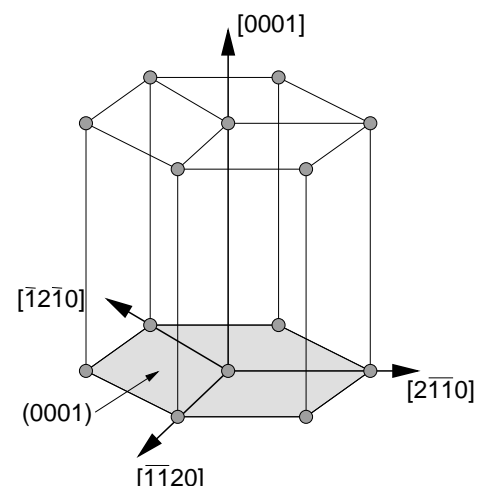

Abbildung 2.1: Hexagonales Gitter. Die Gitterpunkte und die Basisvektoren sind eingezeichnet. Zusätzlich ist die basale (0001)-Ebene schattiert.

parallel zur Richtung [0001] sind, werden prismatische Ebenen genannt. Das Wachstum von GaN-Schichten findet üblicherweise entlang der $c$-Achse statt.

Die basalen (0002)-Doppelebenen bestehen aus einer Ga- und einer N-Ebene, wobei konventionell die Ga-Ebene in [0001]-Richtung liegt. Die in [0001]-Richtung übereinander liegenden Ga- und N-Atome aus zwei benachbarten Doppelebenen können dabei als strukturelle Einheit betrachtet werden, so dass die Struktur sich durch die Stapelfolge ...ABABAB... beschreiben lässt (s. Abb. 2.2). Die metastabile Zinkblende-Phase ( $\beta$-Phase) zeichnet sich im Vergleich dazu durch die Stapelfolge ...ABCABC... in [111]-Richtung aus.

(a)

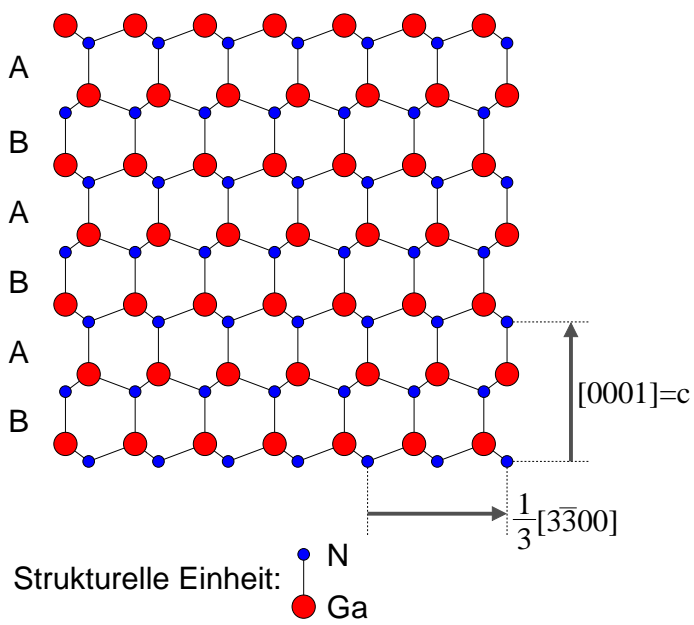

(b)

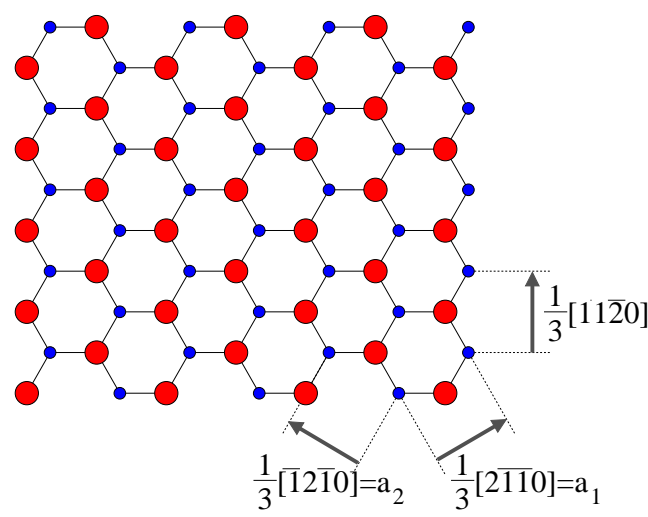

Abbildung 2.2: Struktur von GaN. (a) Projektion auf eine (11200)-Ebene. (b) Projektion einer basalen Doppelebene auf die (0001)-Ebene.

\subsubsection{Ausgedehnte Defekte in Wurtzit-GaN}

Übersichtsartikel, die ausgedehnte Defekte und Versetzungen in GaN behandeln, können in [15] und [16] gefunden werden. Über Stapelfehler und Versetzungen informieren die beiden 
folgenden Abschnitte.

\subsubsection{Stapelfehler}

Stapelfehler lassen sich als zweidimensionale Defekte zunächst durch ihre Ebene charakterisieren. Im wurtzitischen GaN treten sie in zwei verschiedenen kristallographischen Ebenen auf. Basale Stapelfehler (BSF) sind Stapelfehler in der basalen $\{0001\}$-Ebene. Prismatische Stapelfehler (PSF) lassen sich auf den prismatischen $\{2 \overline{11} 0\}$-Ebenen finden.

Basale Stapelfehler fügen lokal in [0001]-Richtung die kubische Stapelfolge ABC in die ansonsten hexagonale Stapelfolge ...ABAB... ein. Dabei werden drei Typen von BSF unterschieden: die beiden intrinsische Typen $\mathrm{I}_{1}$ und $\mathrm{I}_{2}$, sowie der extrinsische Typ E [17]. Der $\mathrm{I}_{1}$-Typ (s. Abb. 2.3a) wird durch das Entfernen einer A-Ebene gefolgt von einer $\frac{1}{3}\langle 10 \overline{1} 0\rangle$ Abscherung der einen Kristallhälfte erzeugt, so dass sich insgesamt die Stapelfolge ...ABABACACA... ergibt (die kubische Stapelfolge ist unterstrichen). Dieser Stapelfehler wird von einer Frank-ShockleyPartialversetzung mit einem Burgersvektor von $\boldsymbol{b}=\frac{1}{6}\langle 20 \overline{2} 3\rangle$ berandet. Der zweite intrinsische Stapelfehler, Typ $\mathrm{I}_{2}$, ergibt sich durch die $\frac{1}{3}\langle 10 \overline{1} 0\rangle$ Abscherung einer Kristallhälfte (s. Abb. 2.3b). Dies führt zur einer Stapelfolge ...ABABCACAC..., und der Defekt wird entsprechend durch eine Shockley-Partialversetzung, Burgersvektor $\boldsymbol{b}=\frac{1}{3}\langle 10 \overline{1} 0\rangle$, beendet. Der extrinsische Stapelfehler, Typ E, entspricht dem Einschub einer zusätzlichen C-Ebene in den perfekten Kristall (Abb. 2.3c). Die sich ergebene Stapelfolge ist ...ABABCABAB..., begrenzt wird dieser Stapelfehler durch Frank-Partialversetzungen mit dem Burgersvektor $\boldsymbol{b}=\frac{1}{2}\langle 0001\rangle$.

Stapelfehler vom extrinsischen Typ E werden selten beobachtet, da sie energetisch ungünstig sind. Durch eine Abscherung einer Kristallhälfte um $\frac{1}{3}\langle 10 \overline{1} 0\rangle$ ist es möglich, diesen Stapelfehler in den energetisch günstigeren $\mathrm{I}_{1}$-Typ zu verwandeln. Eine derartige Umwandlung des $\mathrm{I}_{2^{-}}$ Stapelfehlers in einen anderen Typ ist nicht möglich.

In der Literatur sind die folgenden theoretischen und experimentellen Werte für die Stapelfehlerenergien zu finden (Angaben in $\mathrm{meV} / \AA^{2}$ ):

\begin{tabular}{lcccc} 
Autor & Methode & $\mathrm{I}_{1}$-Typ & $\mathrm{I}_{2}$-Typ & E-Typ \\
\hline \hline Stampfl et al. $[18]$ & LDA & 1.13 & 2.71 & 4.29 \\
Wright [19] & DFT/LDA & 1.16 & 2.55 & 3.95 \\
Suzuki et al. $[$ [20] & Weak-Beam & & $1.37(25)$ & \\
Zakharov et al. $[21]$ & HRTEM & & $2.49(25)$ & \\
\hline
\end{tabular}

Die prismatischen Stapelfehler werden auch Translationsdomänengrenzen genannt. Sie werden vornehmlich durch Umklappen der $\mathrm{I}_{1}$-Stapelfehler in die prismatische Ebene während des Wachstums erzeugt [15]. Es existieren zwei verschiedene atomare Strukturen dieser Stapelfehler. Ein Modell für die erste PSF-Struktur wurde von Blank et al. [22] vorgeschlagen (s. Abb. 2.4a), dieses Modell wird in der Literatur auch teilweise als Amelinckx-Modell bezeichnet. Dieser PSF lässt sich durch einen Verschiebungsvektor von $\frac{1}{6}\langle 20 \overline{2} 3\rangle$ charakterisieren. Dies entspricht dem Verschiebungsvektor des $\mathrm{I}_{1}$-Fehlers, so dass diese Stapelfehler ohne die Bildung von Versetzungen ineinander umklappen können.

Ein Modell für die Struktur des anderen PSF (s. Abb. 2.4b) wurde von Drum et al. vorgeschlagen [23]. Dieses Modell hat einen Verschiebungsvektor von $\frac{1}{2}\langle 10 \overline{1} 1\rangle$. Daher führt das 
(a) $l_{1}-$ Typ

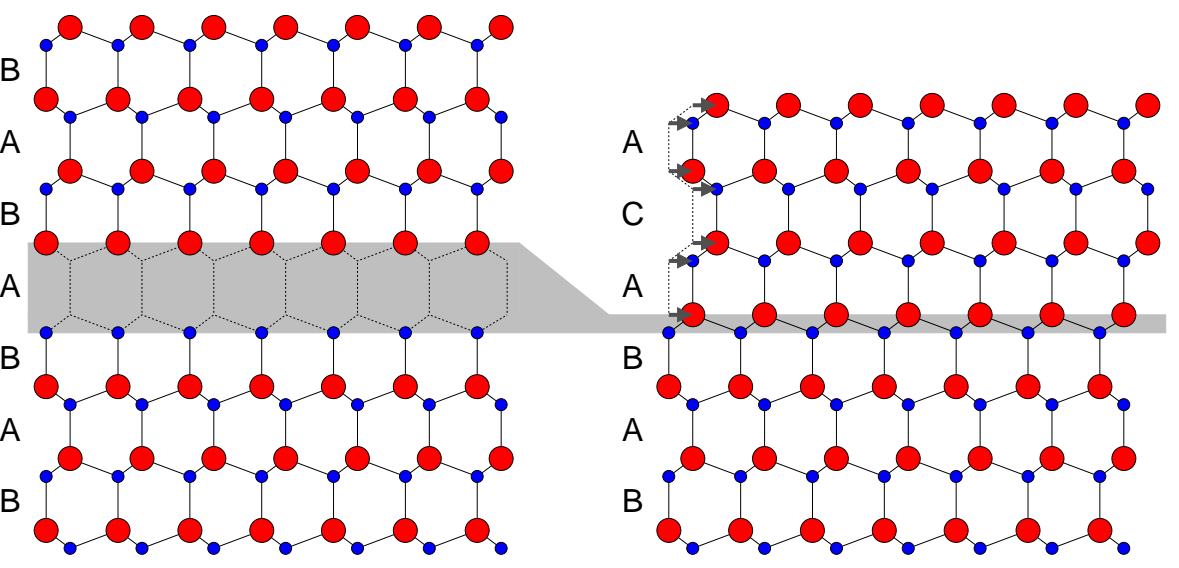

(b) $\mathrm{I}_{2}$-Typ

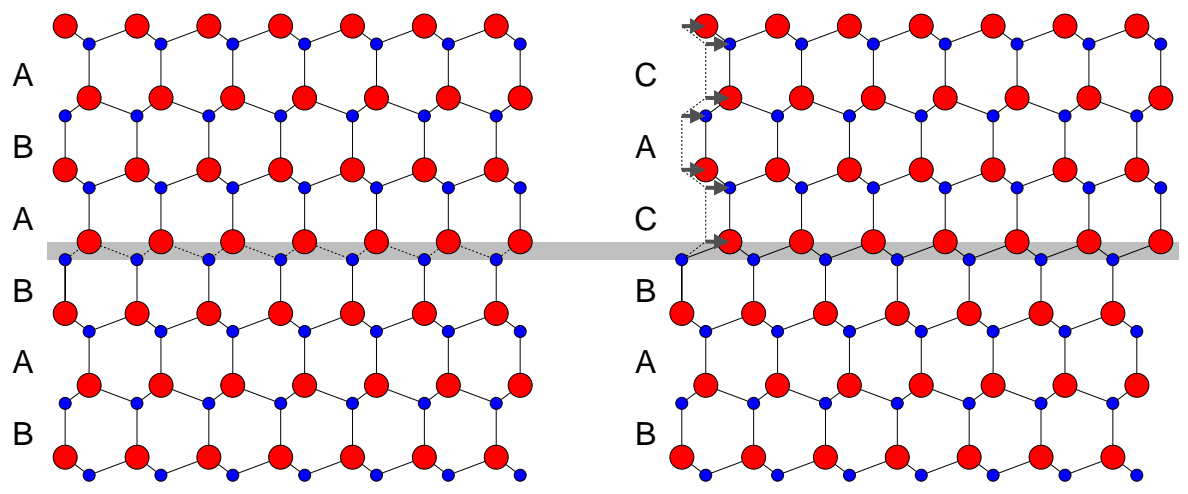

(c) E-Typ
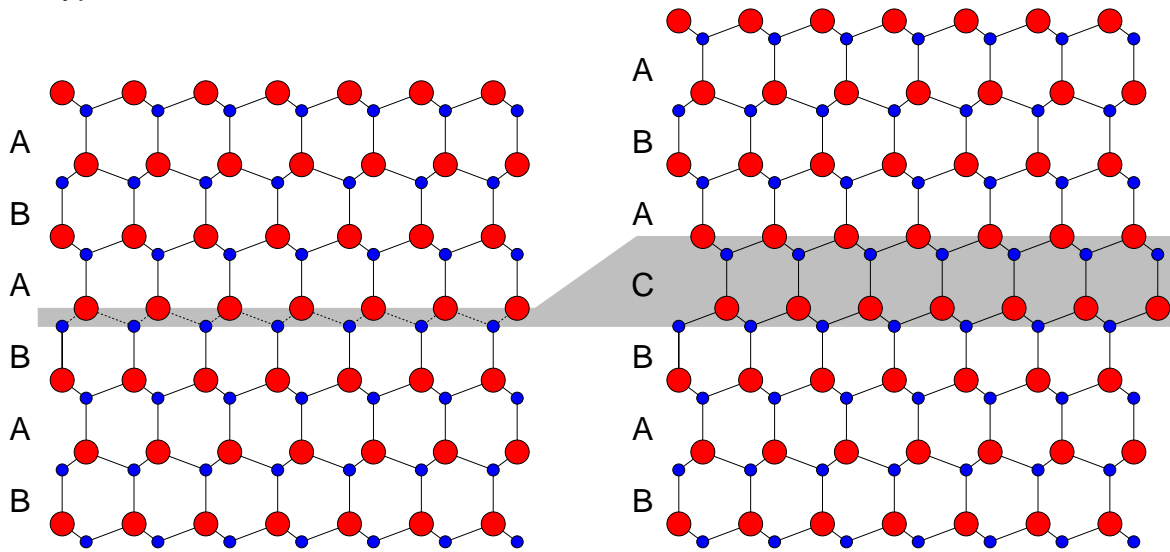

Abbildung 2.3: Atomare Struktur der basalen Stapelfehler in GaN. 
(a)

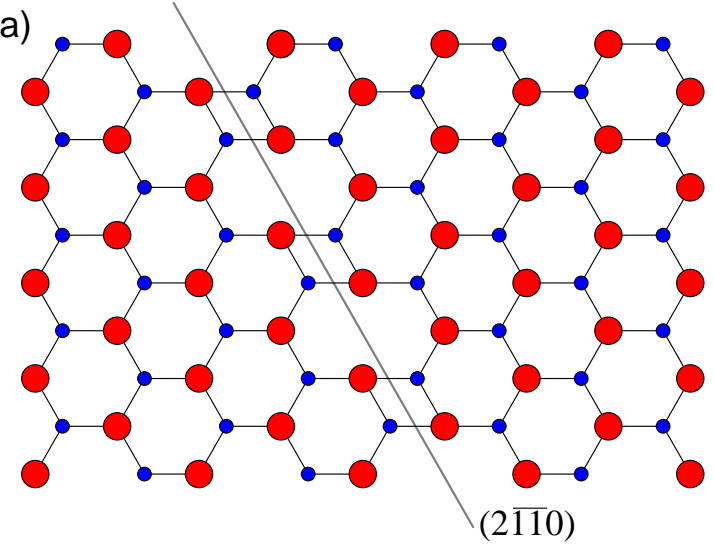

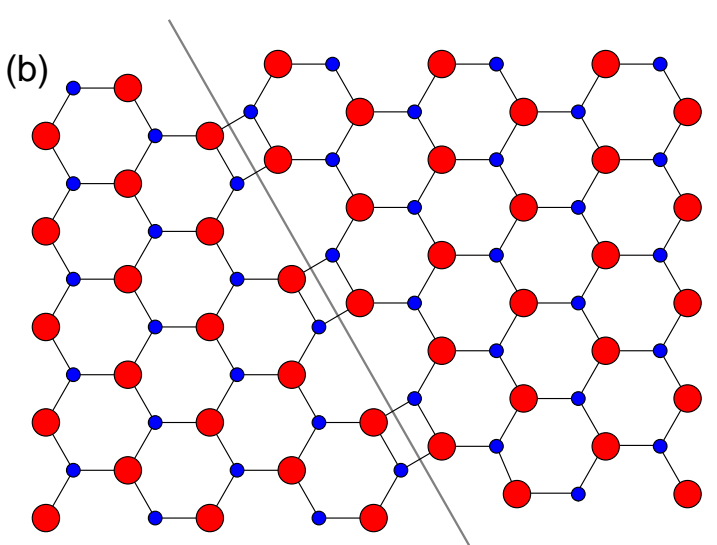

$(2 \overline{110})$

Abbildung 2.4: Prismatische Stapelfehler: (a) Blank-Modell. (b) Drum-Modell.

Umklappen eines $\mathrm{I}_{1}$-Stapelfehlers von der basalen in die prismatische Ebene zur Bildung einer Partialversetzung (,Stairrod“-Versetzung) mit einem Burgersvektor von $\boldsymbol{b}=\frac{1}{6}\langle 10 \overline{1} 0\rangle$.

Northrup hat berechnet, dass das Drum-Modell mit einer Stapelfehler-Energie von $72 \mathrm{meV} / \AA^{2}$ energetisch bevorzugt ist. Er schätzt, dass die Energie des Blank-Modells um etwa $70 \mathrm{meV} / \AA^{2}$ größer ist [24]. Diese energetischen Unterschiede werden durch die Tatsache bestätigt, dass 90\% der beobachteten PSF in auf Saphir gewachsenem GaN vom Drum-Typ sind [25].

\subsubsection{Perfekte Versetzungen}

Die Burgers-Vektoren von perfekten Versetzungen sind in Wurtzit-Kristallen die gleichen wie in $h c p$-Strukturen: $\frac{1}{3}\langle 11 \overline{2} 0\rangle$ ( $a$-Typ), $\langle 0001\rangle$ (c-Typ), und $\frac{1}{3}\langle 11 \overline{2} 3\rangle((a+c)$-Typ).

Die Grenzschichten des GaN zu Substrat oder Pufferschichten liegen in den Basalebenen. Mechanische Spannungen (misfit) in diesen Schichten können über Versetzungen mit Linienelementen in $\langle 11 \overline{2} 0\rangle$-Richtung und Burgersvektoren vom Typ $\boldsymbol{b}=\frac{1}{3}\langle 11 \overline{2} 0\rangle$ abgebaut werden. Die auftretenden Misfit-Versetzungen haben entweder reinen Schrauben- $\left(0^{\circ}\right)$ oder einen $60^{\circ}$ Charakter, je nach Winkel zwischen Linienelement und Burgersvektor.

Neben diesen basalen Versetzungen werden die drei Typen von perfekten Versetzungen mit Linienelementen in [0001]-Richtung beobachtet. Der vorwiegende Teil dieser als Faden-Versetzungen bezeichneten Versetzungen ist vom $a$-Typ, d.h. es handelt sich um reine Stufenversetzungen. Der $c$-Typ, also die Schraubenversetzung, macht einen Anteil von etwa 1\% der Versetzungen aus, der $(a+c)$-Typ ist von gemischtem Charakter, sein Anteil liegt unter $10 \%$. Die Fadenversetzungen treten mit verschiedenen atomaren Kernstrukturen auf. Die Schraubenversetzung kann auch einen leeren Kern haben (Nanotubes) [16].

Der starke Misfit resultiert in einem GaN-Wachstum, das zunächst in kleinen Inseln oder Säulen abläuft. Dabei sind diese Inseln in Winkeln im Bereich von wenigen Bogenminuten gegeneinander verdreht [26]. Beim weiteren Wachstum koaleszieren diese Inseln. Die basalen Misfit-Versetzungen sowie Partialversetzungen, wie sie sich am Rande von Stapelfehlern finden, 
klettern und reagieren beim Verwachsen der Inseln miteinander. Durch das weitere Wachstum werden sie zu den Fadenversetzungen [16, 27].

\subsubsection{Mn dotiertes GaN}

Für technische Spintronik-Anwendungen wäre Ferromagnetismus bei Raumtemperatur eine Voraussetzung. Diesen sagen Dietl et al. mit Hilfe eines Mean-Field-Modells basierend auf einem Zener p-d Austausch für hexagonales GaN und $\mathrm{ZnO}$ voraus, wenn 5 at\% Mn substitutionell auf dem Kationenplatz gelöst sind. Dabei müssen diese Halbleiter hoch p-dotiert $\left(10^{20} / \mathrm{cm}^{3}\right)$ sein, da die magnetische Ordnung durch die Löcher vermittelt wird [8].

Andere theoretische Arbeiten prognostizierten ebenfalls Ferromagnetismus bei Raumtemperatur für 5 at\% Mn-dotiertes GaN: So berechneten Sato et al. mit einem Mean-Field-Modell, dass sich das Mn-Störstellenband durch Doppelaustausch verbreitert [9]. Diese Verbreiterung bringt nur einen Energiegewinn, solange die Fermienergie innerhalb dieses Bandes liegt. Daher ist der magnetische Zustand von der Fermienergie abhängig. Für Lagen der Fermienergie außerhalb des Störstellenbandes sind Antiferromagnetismus (aufgrund von Superaustausch) und Spinglas-Verhalten zu erwarten [28].

In einer neueren Arbeit korrigieren Sato et al. diese Aussage [29]: Der Doppelaustausch wird von Wechselwirkungen zwischen Nächsten-Nachbarn (NN) dominiert, d.h. er ist sehr kurzreichweitig. Dies führt für niedrige Konzentrationen der magnetischen Ionen dazu, dass die magnetische Ordnung nicht über den Kristall perkolieren kann. Zum Vergleich: die Perkolationsschwelle liegt für NN in fcc-Strukturen bei Konzentrationen von 20 at\%. Die MeanField-Modelle unterschätzen dieses Perkolationsproblem. Werden die Berechnungen stattdessen mit Monte-Carlo-Simulationen durchgeführt, ergeben sich für 5 at\% Mn-Dotierung CurieTemperaturen unterhalb von $50 \mathrm{~K}$.

Unterschiedliche Aussagen bzgl. der magnetischen Eigenschaften von Mn-dotiertem GaN gibt es nicht nur von theoretischer, sondern auch von experimenteller Seite: So wurde von verschiedenen Gruppen ferromagnetisches Verhalten mit Curie-Temperaturen von $10 \mathrm{~K}$ bis $940 \mathrm{~K}$ beobachtet [30, 31, 32, 33, 34, 35], während andere Gruppen Spinglas-Verhalten [36, 37] und auch Antiferromagnetismus [38] berichten. Diese uneinheitlichen Ergebnisse haben ihre Ursache teilweise darin, dass viele Gruppen die Ergebnisse der magnetischen Messungen unabhängig davon berichten, in welcher Form das Mn tatsächlich in das GaN eingebaut wurde [11]. Prinzipielle Möglichkeiten für den Einbau sind:

- GaN mit gelöstem Mn. Hier kann das Mn interstitiell oder substitutionell eingebaut werden. Im Fall von substitutionell eingebautem Mn kann tatsächlich von einer MnDotierung gesprochen werden. Auch die Art der Leitfähigkeit des GaN muss beachtet werden, da der theoretisch vorhergesagte Ferromagnetismus nicht bei n-dotiertem GaN eintreten soll.

- Ausscheidungen. Für höhere Mn-Konzentrationen berichten viele Gruppen von Ausscheidungen, die sie mittels Röntgendiffraktometrie (XRD) nachwiesen. Allerdings lassen sich nur wenige Nanometer große Ausscheidungen nicht mehr mittels XRD nachweisen. So fanden z.B. Dhar et al. [37] mittels TEM Ausscheidungen in Schichten, die nach XRDMessungen einphasig waren. 
- Mn-Cluster, d.h. Ansammlungen von wenigen Mn-Atomen. Berechnungen zeigen, dass es für substitutionelle Mn-Atome energetisch günstig ist, sich um N-Atome anzusammeln, sowie dass isolierte $\mathrm{Mn}_{x} \mathrm{~N}$-Cluster sehr große magnetische Momente im Bereich bis zu $22 \mu_{B}$ haben können [39, 40]. Der Einfluss dieser Cluster auf die Curie-Temperatur der GaN:Mn-Schichten ist von theoretischer Seite noch nicht eindeutig geklärt [41].

Die Grenzen zwischen diesen Arten des Mn-Einbaus sind sicherlich fließend. Größere Cluster können auch als kleine Ausscheidungen angesehen werden. Zufällig auf Ga-Plätzen gelöstes Mn bildet offensichtlich allein aus statistischen Gründen schon Cluster, so sind beispielsweise, bei 6 at\% zufällig verteiltem $\mathrm{Mn}$ schon $27 \%$ der Mn-Atome in $\mathrm{Mn}_{2} \mathrm{~N}$-Clustern zu finden [41].

Verschiedene Gruppen beobachteten Ausscheidungen, die auftreten, wenn die Mn-Konzentration bestimmte Werte $(<10$ at\%) überschreitet, wobei diese Werte von Herstellungsbedingungen (Methode, Wachstumstemperatur) und Schichtdotierung [42] abhängen. Für die in dieser Arbeit untersuchten Proben wurden Ausscheidungen bei Konzentrationen $>5$ at\% beobachtet [43]. Die Ausscheidungen wurden meist als $\mathrm{GaMn}_{3} \mathrm{~N}$ identifiziert. Aus XRD-Messungen allein lässt sich diese Phase nicht eindeutig identifizieren, da die Gitterkonstanten für die perowskitische Phase $\mathrm{Ga}_{1-x} \mathrm{Mn}_{3+x} \mathrm{~N}$ zwischen $0.3871 \mathrm{~nm}(\mathrm{x}=0)$ und $0.3885 \mathrm{~nm}(\mathrm{x}=1)$ variieren, und dieser Unterschied in den Bereich der XRD-Auflösung fällt. Dabei ist $\mathrm{GaMn}_{3} \mathrm{~N}$ antiferromagnetisch und $\mathrm{Mn}_{4} \mathrm{~N}$ ferrimagnetisch [44, 45, 46]. Es werden daher weitere Messungen zur Phasenbestimmung benötigt. Kim et al. deuten die Phase auf Basis von magnetischen Messungen als $\mathrm{GaMn}_{3} \mathrm{~N}$ [35]. Giraud et al. schließen aus Messungen der RöntgenabsorptionskantenFeinstruktur (,Extended X-Ray Absorption Fine Structure“; EXAFS) auf GaMn ${ }_{3} \mathrm{~N}$ [47]. Auch andere $\mathrm{Ga}_{x} \mathrm{Mn}_{y}$ Phasen wurden gefunden [32, 33, 48], teilweise nach Auslagerung der Proben $[32,48]$.

Auch die magnetischen Messungen in Proben, in denen keine Ausscheidungen mittels XRD gefunden wurden, zeigen teilweise die Koexistenz von verschiedenen magnetischen Beiträgen [31, 32, 33, 34], wobei die Autoren größtenteils die ferromagnetischen Anteile als intrinsische Beiträge der Mn-dotierten GaN-Schicht deuten, aber nicht näher belegen, dass sie nicht von anderen Phasen stammen. Anzumerken ist auch [36], dass Curie-Temperaturen bei Raumtemperatur oder höher vorwiegend in $n$-GaN oder nichtleitendem GaN gefunden wurden $[33,34,35]$. Verschiedene Autoren deuten dies heute so, dass die ferromagnetischen Beiträge vorwiegend von Clustern und Ausscheidungen stammen [28, 49], die GaN-Schichten selbst allerdings Spinglas-Verhalten zeigen [37].

In GaN-Schichten mit gelöstem Mn, also ohne Ausscheidungen, ist die Frage zu klären, in welchem Umfang es substitutionell auf dem Kationenuntergitter eingebaut wird. Für interstitielles Mn sind andere elektrische Eigenschaften zu erwarten [50]. In Mn-dotiertem GaAs wurde mittels winkelaufgelöstem Rutherford-Backscattering (RBS) nachgewiesen, dass sich das Mn auch teilweise auf interstitiellen Plätzen befindet, was zu einer deutlichen Verringerung der Curie-Temperatur führt [51]. Mit demselben Messverfahren wurde beobachtet, dass $\mathrm{Cr}$ in GaN, je nach Wachstumsbedingungen, nur zu $78-90 \%$ auf dem Ga-Platz eingebaut wird [52]. Der Gitterplatz von Mn in GaN wurde meist nur durch EXAFS- oder "Near-Edge X-Ray Absorption Fine Structure" (NEXAFS)-Messungen der Röntgenabsorptionskanten bestimmt. Die Messungen ergaben, dass das Mn ,größtenteils“ auf dem Ga-Platz sitzt, ohne dass eine nähere Quantifizierung vorgenommen wurde [53, 54, 55]. Einzig Kuroda und Mitarbeiter [56] haben vor kurzem mittels winkelaufgelöster RBS- und winkelaufgelöster "Particle Induced X- 
Ray Emission“ (PIXE)-Messungen nachgewiesen, dass das Mn zu 87\% bis $94 \%$ substitutionell gelöst ist.

Methoden wie EXAFS, die sensitiv auf die chemische Umgebung der Mn-Atome sind, wurden bisher noch nicht eingesetzt, um zu überprüfen, ob das Mn statistisch in den GaN-Schichten verteilt ist oder sich bevorzugt in Clustern anhäuft.

Eine weitere Übersicht über theoretische und experimentelle Ergebnisse, gerade in Hinblick auf die Ursachen des beobachteten Ferromagnetismus, ist in den Artikeln von Liu et al. [11] und Pearton et al. [10] zu finden.

Diese Vielzahl an unterschiedlichen experimentellen Befunden macht deutlich, dass die Ergebnisse stark von der Mikrostruktur der Probe abhängen. Daher müssen die Proben bzgl. des Mn-Einbaus sehr genau charakterisiert werden, da die beobachteten magnetischen Eigenschaften nicht nur vom GaN:Mn, sondern auch von Ausscheidungen oder Clustern herrühren können. XRD-Messungen allein können nicht mit letzter Sicherheit feststellen, ob Ausscheidungen vorliegen, wenn diese wenige Nanometer groß sind. Auch die Phase der Ausscheidungen kann nicht eindeutig bestimmt werden, da teilweise weitere chemische Analysen notwendig werden. Messverfahren wie EXAFS und winkelaufgelöstes RBS können zur näheren Untersuchung der Art des Mn-Einbaus benutzt werden, allerdings mitteln diese Techniken über große Probenbereiche und können nicht die Mikrostruktur der Probe und Wechselwirkungen des Mn-Einbaus mit strukturellen Defekten untersuchen.

Hier sind Untersuchungen mittels TEM, zusammen mit analytischen Methoden wie energiedispersiver Röntgenanalyse (EDX) oder „Electron Energy Loss Spectroscopy“ (EELS), am Besten geeignet. HRTEM kann genutzt werden, um strukturelle Defekte zu lokalisieren und zu identifizieren. Die analytischen Messungen können mit hinreichend hoher Ortsauflösung vorgenommen werden, um ihre Ergebnisse mit der Probenstruktur zu korrelieren. Wie in Abschnitt 7 gezeigt wird, sind mittels winkelaufgelöster Messungen auch hier Gitterplatz-Bestimmungen möglich.

\subsection{Moderne Transmissionselektronenmikroskopie}

Hochaufösende Elektronenmikroskopie erlaubt es, Materialien ortsaufgelöst und quantitativ zu charakterisieren. Verschiedene Techniken können angewandt werden, um Schichtgeometrien, Zusammensetzungen, elastische Verzerrungen und Gitterbaufehler zu untersuchen. Je nach verwendeter Technik können dabei Ortsaufösungen unterhalb von $1 \AA$ erreicht werden.

Es lassen sich zwei unterschiedliche Ansätze unterscheiden: Zum einen die abbildende Elektronenmikroskie, die es erlaubt, hochaufgelöste Abbildungen von Proben anzufertigen, die sich weiter in Hinblick auf die Struktur, d.h. die Atompositionen, untersuchen lassen. Abschnitt 2.2.1 gibt einen Überblick über den aktuellen Stand der Technik auf diesem Gebiet. Der andere Ansatz ist die analytische Elektronenmikroskopie, die inelastische Streuprozesse innerhalb der Probe ausnutzt, um chemische Informationen über die Probe zu gewinnen. Dieser Ansatz wird in Abschnitt 2.2.2 vorgestellt. Dabei lassen sich zu einem gewissen Grad die beiden Ansätze kombinieren, da sich die analytischen Techniken, wie EELS und EDX, parallel zur Abbildung anwenden lassen.

Ausführlichere Darstellungen über die Methoden der modernen TEM sind in der Literatur zu finden $[57,58,59]$. 


\subsubsection{Abbildende Transmissionselektronenmikroskopie}

Die erreichbare Punktauflösung in HRTEM-Abbildungen ist bei Mittelspannungsgeräten (Beschleunigungsspannung 200-300 kV) vorwiegend durch die optische Delokalisierung auf etwa $2 \AA$ beschränkt. Die Ursache der Delokalisierung liegt in den großen Linsenfehlern der Elektronenlinsen, insbesondere der großen sphärischen Aberration, die durch die ÖffnungsfehlerKonstante $C_{\mathrm{s}}$ quantifiziert wird. Für die Beobachtung von Kristalldefekten reicht eine Auflösung um $2 \AA$ oft nicht aus, da sie nur die Abbildung der niedrig indizierten Kristallreflexe erlaubt. So lassen sich z.B. in Si nur (111)-Reflexe innerhalb dieser Auflösung abbilden, eine Auflösung von $1 \AA$ erlaubt bereits die Abbildung von sechs zusätzlichen Arten von Reflexen [58].

Zwar ermöglicht die hohe Kohärenz der Feldemissions-Elektronenquellen („Field Emission Gun“; FEG) auch höhere Raumfrequenzen durch das Mikroskop zu übertragen, allerdings tritt eine starke Veränderung der Abbildung durch die optische Delokalisierung auf, was eine direkte Interpretation der entstehenden Abbildungen im Allgemeinen unmöglich macht.

Eine Erhöhung der Beschleunigungsspannung zur Verkürzung der Elektronenwellenlänge und somit zur Verbesserung der Auflösung ist aufgrund der Probenschädigung bei höheren Spannungen keine echte Alternative. Daher werden heutzutage verschiedene Ansätze genutzt, um Auflösungen im Bereich von einem Ångström zu erreichen:

- $C_{\mathrm{s}}$-Korrektur der Objektivlinse. Während Linsenfehler wie Astigmatismus und axiale Koma schon lange korrigierbar sind, sind erst in jüngerer Zeit kommerzielle Korrektoren für die sphärische Aberration verfügbar geworden. Der $C_{\mathrm{s}}$-Korrektor behebt nicht nur das Problem der optischen Delokalisierung, sondern vermeidet auch Kompromisse, die bei $C_{\mathrm{s}}$-optimierten Mikroskopen eingegangen werden müssen. So wird z.B. wieder ein größerer Kippbereich der Probe erreicht, da der Abstand zwischen Probe und den Objektivlinsenpolschuhen vergrößert werden kann. Gegenüber den folgenden Methoden hat ein $C_{\mathrm{s}}$-korrigiertes Mikroskop den Vorteil, dass delokalisierungsfreie Abbildungen mit viel geringerem Aufwand möglich sind.

- „Off-Axis“-Holographie. Diese Technik erlaubt es, nicht nur Intensitäten aufzuzeichnen, sondern die gesamte komplexwertige Bildwelle, d.h. Amplitude und Phase, zu rekonstruieren. Die Effekte der Linsenfehler können in der komplexen Bildwellenfunktion ohne Informationsverlust numerisch korrigiert werden. Ein weiterer Vorteil dieser Technik ist, dass es möglich ist, aus den zusätzlich gewonnen Phaseninformationen die Größe elektrischer und magnetischer Felder innerhalb der Probe selbst zu bestimmen. Ein weiterer positiver Nebeneffekt ist eine nahezu ideale Energiefilterung. Nachteilig an der Holographie ist, dass es sich um ein sehr aufwendiges Verfahren handelt [60].

- Defokusserienrekonstruktion. Die Defokusserienrekonstruktion ist ein numerisches Verfahren, um aus unter unterschiedlichen Abbildungsbedingungen aufgenommenen Abbildungen die Bildwelle zu konstruieren. Die Effekte der Linsenfehler können in der Bildwelle, wie bei der Holographie, numerisch korrigiert werden. Im Gegensatz zur Holographie lassen sich mit dieser Technik allerdings keine tieffrequenten Bildanteile rekonstruieren. Diese tragen zwar nicht zur hochauflösenden Abbildung bei, werden aber benötigt, um Felder in der Probe zu messen. Ebenso wie die Holographie ist dieses ein sehr aufwendiges Verfahren (s. Kapitel 4). 
- „High Angle Annular Dark Field - Scanning TEM“ (HAADF-STEM). Elektronen, die unter großen Winkeln aus der Probe gestreut werden, d.h. Elektronen die zum HAADFSignal beitragen, sind vorwiegend nahe am Atomkern inkohärent gestreut worden. Das hat zur Folge, dass das HAADF-Signal sehr sensitiv auf die Atompositionen ist. Aufgrund der großen Messwinkel tragen Elektronen, die Bragg-Streuung erfahren haben, kaum zu diesem Signal bei. Durch eine $C_{\mathrm{s}}$-Korrektur im Beleuchtungssystem lässt sich der Elektronenstrahl auf Durchmesser von unterhalb $1 \AA$ fokussieren [61]. Ein Abrastern der Probe mit dem Strahl erlaubt eine hochaufösende Abbildung mit dem HAADFSignal. Auch chemische Informationen lassen sich mit dieser Technik gewinnen, da das HAADF-Signal auch abhängig von der Kernladung der Atome ist.

Mit den oben genannten Methoden ist es möglich, die Punktauflösung des Mikroskops wesentlich zu verbessern. Zeitliche Schwankungen in den Strom- und Spannungsversorgungen begrenzen dann durch die chromatischen Aberrationen der Objektivlinse die Auflösung. Aktuelle Entwicklungen gehen dahin, auch Korrektoren für den chromatischen Fehler $C_{c}$ zu entwickeln, um eine noch bessere Auflösung zu erreichen [62].

In dieser Arbeit wird zum Erreichen der höchst möglichen Auflösung des verwendeten Mikroskops die Defokusserienrekonstruktion eingesetzt, da sie die einzige Technik ist, die sich ohne apparative Änderungen einsetzen lässt.

\subsubsection{Analytische Transmissionselektronenmikroskopie}

Die zwei hauptsächlich in der analytischen Elektronenmikroskopie eingesetzten Techniken sind EDX und EELS. Während es im Allgemeinen bessere analytische Verfahren gibt, haben die Techniken der analytischen TEM den Vorteil einer sehr hohen Ortsauflösung. Diese ist dabei im Wesentlichen durch die geringen Zählraten begrenzt, die mit hoher Ortsauflösung einhergehen.

Beide Techniken nutzen inelastische Streuprozesse in der Probe, um Aussagen über die chemische Zusammensetzung der Probe zu treffen. Bei der Röntgenanalyse durch EDX wird die charakteristische Röntgenstrahlung der Probe analysiert. Die inneren Schalen der Atome werden durch den Elektronen-Primärstrahl ionisiert und relaxieren u.a. durch Aussenden der charakteristischen Röntgenstrahlung. Ein Röntgendetektor nahe an der Probe misst diese Strahlung.

Bei EELS hingegen wird die Intensität des Elektronenstrahls nach Durchlaufen der Probe in Abhängigkeit von seiner Energie gemessen. Aufgrund der thermischen Emission der Elektronen aus der Quelle und der begrenzten Stabilität der Beschleunigungsspannung liegt die Energiebreite des Elektronenstrahls vor Durchlaufen der Probe allerdings im Bereich von $1 \mathrm{eV}$. Eine höhere Energieauflösung kann jedoch durch den Einsatz von Monochromatoren erreicht werden.

Das EDX- und EELS-Signal werden dabei integral über die gesamte beleuchtete Fläche der Probe gemessen. Wird das Mikroskop als STEM betrieben, d.h. wird der Elektronenstrahl auf einen kleinen Bereich fokussiert und über die Probe gerastert, so können die chemischen Informationen parallel zur Rasterabbildung gewonnen werden. EELS ermöglicht auch eine andere Messweise, indem energiegefilterte Abbildungen für bestimmte Verlustenergien aufgenommen werden. Diese energieselektiven Abbildungen können genutzt werden, um z.B. bestimmte Atomsorten zu lokalisieren. 
EELS hat noch weitere Vorteile gegenüber EDX: So sind die Messzeiten kürzer bzw. die Signale stärker, da der Elektronenstrahl im Wesentlichen vorwärts und somit in den Detektor gestreut wird, die charakteristische Röntgenstrahlung wird hingegen in alle Richtungen ausgesendet und der Detektor deckt nur einen bestimmten Raumwinkel ab. Bei leichten Elementen relaxieren ionisierte Atome stärker über Auger-Prozesse als über Röntgenemission. Der Ionisierungsenergieverlust im Elektronenstrahl, der von EELS detektiert wird, tritt bei beiden Prozessen auf, so dass auch hier größere Signale als bei EDX erreichbar sind. Neben Informationen über die Anteile der in den Proben vorhandenen Atomsorten lassen sich aus der Analyse der Absorptionskantenfeinstruktur („Energy Loss Near Edge Spectrum“; ELNES) auch Erkenntnisse über die chemische Umgebung sowie die Valenzzustände der einzelnen Atomsorten gewinnen (vergleichbar zu EXAFS und NEXAFS). Auch der Niedrigverlustbereich $(<10 \mathrm{eV})$ des EELS-Spektrums lässt sich analog zu optischen Absorptionsmessungen interpretieren.

Da beide Techniken auf inelastischen Prozessen beruhen, die zum größten Teil an den Atomen der Probe lokalisiert sind, lassen sich prinzipiell beide Techniken nutzen, um mittels winkelabhängiger Messungen Informationen über die Gitterplatz-Besetzungen innerhalb der Einheitszelle zu gewinnen. Diese Messmethode wird allerdings vorwiegend bei EDX angewandt. In dieser Arbeit wird sie genutzt, um den Gitterplatz des Mn im GaN zu bestimmen (s. Kapitel 7).

Der aparative Aufwand für EELS-Messungen ist höher, da das Mikroskop noch zusätzlich mit Energiefiltern ausgestattet werden muss, und für hohe Energieauflösung auch mit einem Monochromator. Aus diesem Grund wird in der vorliegenden Arbeit nur mit EDX-Analysen gearbeitet.

\section{EDX-Analyse}

In diesem Abschnitt wird eine knappe Einführung in die EDX-Analyse gegeben. Ausführlichere Darstellungen finden sich in der Literatur, z.B. in [57].

Bei der EDX-Analyse wird das Spektrum der aus der Probe emittierten Röntgenstrahlung gemessen. Diese Strahlung setzt sich additiv aus zwei Anteilen zusammen: der kontinuierlichen Bremsstrahlung, deren Intensität mit zunehmender Röntgenenergie abnimmt, und der charakteristischen Röntgenstrahlung, die nur bei bestimmten Energien auftritt, die für das Element charakteristisch sind. Beispiele für solche Spektren sind in Abb. 6.5 zu sehen.

Für eine qualitative Auswertung der Messungen kann die gemessene Röntgenintensität innerhalb eines Energiefensters um die charakteristische Röntgenlinie herum genutzt werden. Diese Auswertung wird z.B. verwendet, um zweidimensionale Karten der Elementverteilung aufzunehmen.

Für eine quantitative Auswertung nach Cliff-Lorimer [63] wird zuerst der Hintergrund, d.h. die Bremsstrahlung, vom Spektrum abgezogen. Desweiteren werden quantitative Aussagen nur über die Intensitätsverhältnisse der verschiedenen Linien gemacht. Im Gegensatz zu den absoluten Intensitäten haben Messbedingungen, wie z.B. der Winkel zwischen Probe und Detektor oder die Probendicke, keinen Einfluss auf diese Verhältnisse. Die Verhältnisse werden weiterhin mit röntgenlinienabhängigen Korrekturfaktoren (den sog. Cliff-Lorimer-Faktoren) korrigiert. Diese Faktoren berücksichtigen Einflüsse wie z.B. die Röntgenfluoreszenz der charakteristischen Linie, Detektoreffizienzen oder Absorptionen im Detektorfenster und sind für 
den benutzten Detektor tabelliert. Das so korrigierte Intensitätsverhältnis entspricht dem Konzentrationsverhältnis der auftretenden Elemente. 


\section{Hochaufgelöste Transmissionselektronenmikroskopie}

\subsection{Abbildung durch das Elektronenmikroskop}

Die Bildentstehung in einem hochauflösenden Elektronenmikroskop ist schematisch als Strahlengang in Abb. 3.1 gezeigt. Die einlaufende, ebene Welle $\psi_{0}$ wird durch Streuprozesse im Objekt verändert. Dieser Prozess muss im Allgemeinen quantenmechanisch beschrieben werden. Hierfür sei auf die entsprechende Literatur verwiesen (z.B. [57] oder [64]). Die austretende Welle auf der Rückseite des Objektes wird als Objektwellenfunktion $\psi$ bezeichnet. Simulationen erlauben es, diese Objektwelle aus Strukturmodellen zu berechnen (s. Abschnitt 3.3). Für dünne, amorphe Objekte lässt sich die Probe als reines Phasenobjekt nähern (s. Abschnitt 3.3.1), was eine einfache Beschreibung des Streuprozesses erlaubt.

Der nachfolgende Abbildungsprozess lässt sich mit den Methoden der Optik beschreiben. Eine scharfe Abbildung entsteht bei Fokussierung auf die Rückseite der Probe (Gaußscher Fokus). Allerdings wird in der Elektronenmikroskopie meist eine andere Ebene scharf gestellt. Der Abstand dieser scharfgestellten Ebene von der Rückseite wird als Defokus $D$ bezeichnet. Dabei wird von Unterfokus gesprochen, wenn die fokussierte Ebene in/vor der Probe liegt (hier $D<0)$ und von Überfokus, wenn sie dahinter liegt $(D>0)$. Durch das Defokussieren wird es möglich, einen Phasenkontrast zu erzielen (s. Abschnitt 3.1.2.2). Dieser wird nötig, da die untersuchten Objekte vorwiegend Phasenobjekte sind und daher wenig Amplitudenkontrast aufweisen, ähnlich zu durchsichtigen Objekten in der Lichtmikroskopie.

Das Wellenfeld in der fokussierten Ebene wird in die Bildebene abgebildet, wobei die Welle noch von Linsenfehlern verändert wird. In der Bildebene wird die Intensität der Bildwellenfunktion $\psi_{B}$ ortsaufgelöst mit einer CCD-Kamera oder einer Photoplatte gemessen.

Im allgemeinen Fall besteht ein komplexer nichtlinearer Zusammenhang zwischen der aufgenommenen Abbildung in der Bildebene und der Objektwelle an der Rückseite der Probe. Dieser Abbildungsprozess selbst wird im Abschnitt 3.1.2.1 ausführlich besprochen. Seine Komplexität hat mehrere Ursachen:

- Die Elektronenlinsen haben starke Linsenfehler, die zur Veränderung von Merkmalen der Objektwelle in der Bildwelle führen. Diese Veränderung wird als optische Delokalisierung $^{1}$ bezeichnet. Die Linsenfehler werden in Abschnitt 3.1.1 ausführlicher beschrieben.

\footnotetext{
${ }^{1}$ Der Begriff „Delokalisierung“ wird in dieser Arbeit zusätzlich noch in einem anderen Zusammenhang verwendet (s. Abschnitt 7.1.2). Um Verwechslungen zu vermeiden, wird der Begriff „optische Delokalisierung“" benutzt, wenn die Veränderungen der Bildwelle bzw. Abbildung gemeint sind, die aufgrund von Linsenfehlern auftreten.
} 


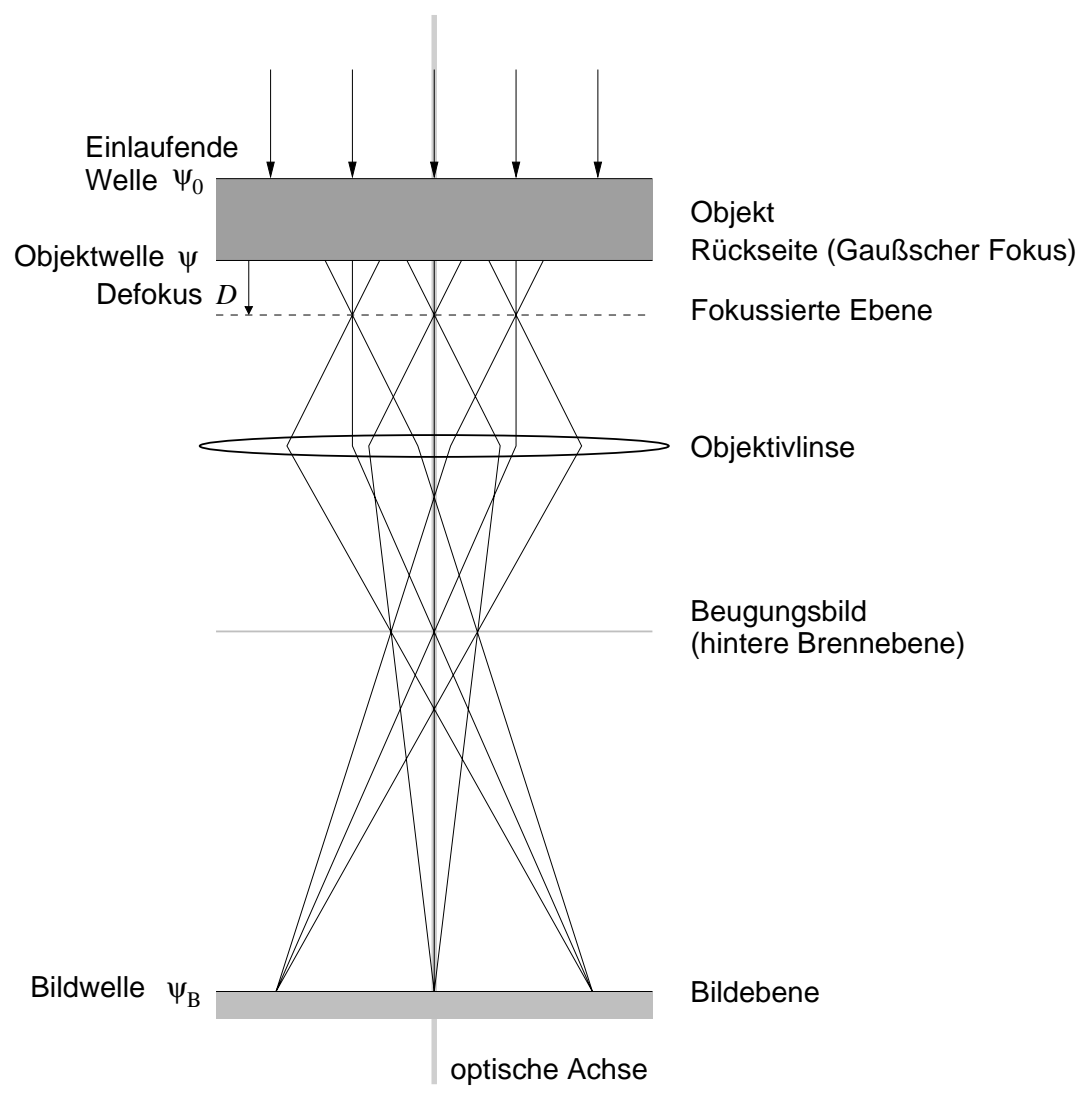

Abbildung 3.1: Schematische Darstellung des Strahlengangs für die Abbildung in einem Elektronenmikroskop. Die einlaufende Welle $\psi_{0}$ wird durch das Objekt verändert. An der Objektrückseite tritt die Objektwelle $\psi$ aus. Die Ebene im Abstand $D$ (Defokus) von dieser Rückseite wird durch die Objektivlinse in die Bildebene abgebildet. Dabei wird die Welle durch die Linsenfehler verändert. 
- Da die Intensitäten der Bildwelle aufgezeichnet werden, ist die Abbildung proportional zum Betragsquadrat der Wellenfunktion.

- Die eingeschränkte Kohärenz der Elektronenwelle muss entsprechend berücksichtigt werden. Im Gegensatz zu Lichtmikroskopen, wo im Sinne der Abbeschen Theorie der Öffnungswinkel der Objektivlinse die Auflösung beschränkt [65], ist es die eingeschränkte zeitliche Kohärenz, die ein Mittelspannungs-Elektronenmikroskop in seiner Auflösung limitiert.

Als Objektwellenrekonstruktion werden Verfahren bezeichnet, die es ermöglichen die Objektwelle selbst zu rekonstruieren. Wird nur die Phase der Objektwellenfunktion betrachtet, so wird auch von der Phasenrekonstruktion gesprochen. Der Rekonstruktionsvorgang ist in zwei Schritte aufteilbar: Als erstes muss die Bildwelle ermittelt werden. Dies geschieht mit Hilfe holographischer Methoden, die die komplexe Bildwellenfunktion rekonstruieren. Eine Übersicht über derartige Verfahren ist bei Cowley [66] zu finden. Als holographische Methode wird in dieser Arbeit die Defokusserienrekonstruktion benutzt. Hier ist die Idee, die Bildwelle aus einer Reihe von 10-20 Einzelabbildungen zu rekonstruieren, indem durch Änderung des Defokus zwischen den einzelnen Aufnahmen der Abbildungsvorgang des Mikroskops in einer kontrollierten Weise verändert wird. In Abschnitt 3.2 wird diese Methode ausführlicher dargestellt.

Der zweite Schritt ist die numerische Korrektur der Linsenfehler, um aus der Bildwelle die Objektwelle innerhalb der Auflösung des Mikroskops wiederzuerlangen. Dazu müssen die genauen Größen der einzelnen Linsenfehler bekannt sein. Methoden, die Linsenfehler bei einem Elektronenmikroskop zu messen, werden in Abschnitt 3.4 beschrieben.

\subsubsection{Linsenfehler}

Linsenfehler werden beschrieben durch den Abstand zwischen der realen (fehlerhaften), durch die Linse erzeugten Wellenfront und der idealen Wellenfront, die eine fehlerfreie Linse erzeugen würde (Abb. 3.2). Der Abstand dieser Wellenfronten entspricht einer Phasenverschiebung, beschrieben durch die Aberrationsfunktion $\chi(\boldsymbol{q})$, in Abhängigkeit des Beugungswinkels $\sin \Theta \approx \Theta=\lambda q$, unter dem ein Elektronenstrahl der Wellenlänge $\lambda$ die Gegenstandsebene verlässt [67]. Dabei wird $\chi(0)=0$ gesetzt, d.h. axiale Strahlen erfahren keine Aberrationen. Im allgemeinen Fall ist die Aberrationsfunktion auch vom Ort in der Gegenstandsebene abhängig. Diese Abhängigkeit wird allerdings in der Elektronenmikroskopie vernachlässigt (isoplanatische Näherung).

Der Effekt der Linsenfehler ist eine Verschmierung der Objektwelle. Die Ursache dafür lässt sich in Abb. 3.2 nachverfolgen: Obwohl alle Strahlen vom gleichen Punkt ausgehen, treffen sie abhängig vom Winkel $\Theta$ an verschiedenen Punkten auf die Bildebene.

Die Aberrationsfunktion mit den in der Elektronenmikroskopie relevanten Linsenfehlern lautet in kartesischen Koordinaten $\boldsymbol{q}=\left(q_{x}, q_{y}\right)$ für axiale Beleuchtung, d.h. Beleuchtung entlang der 


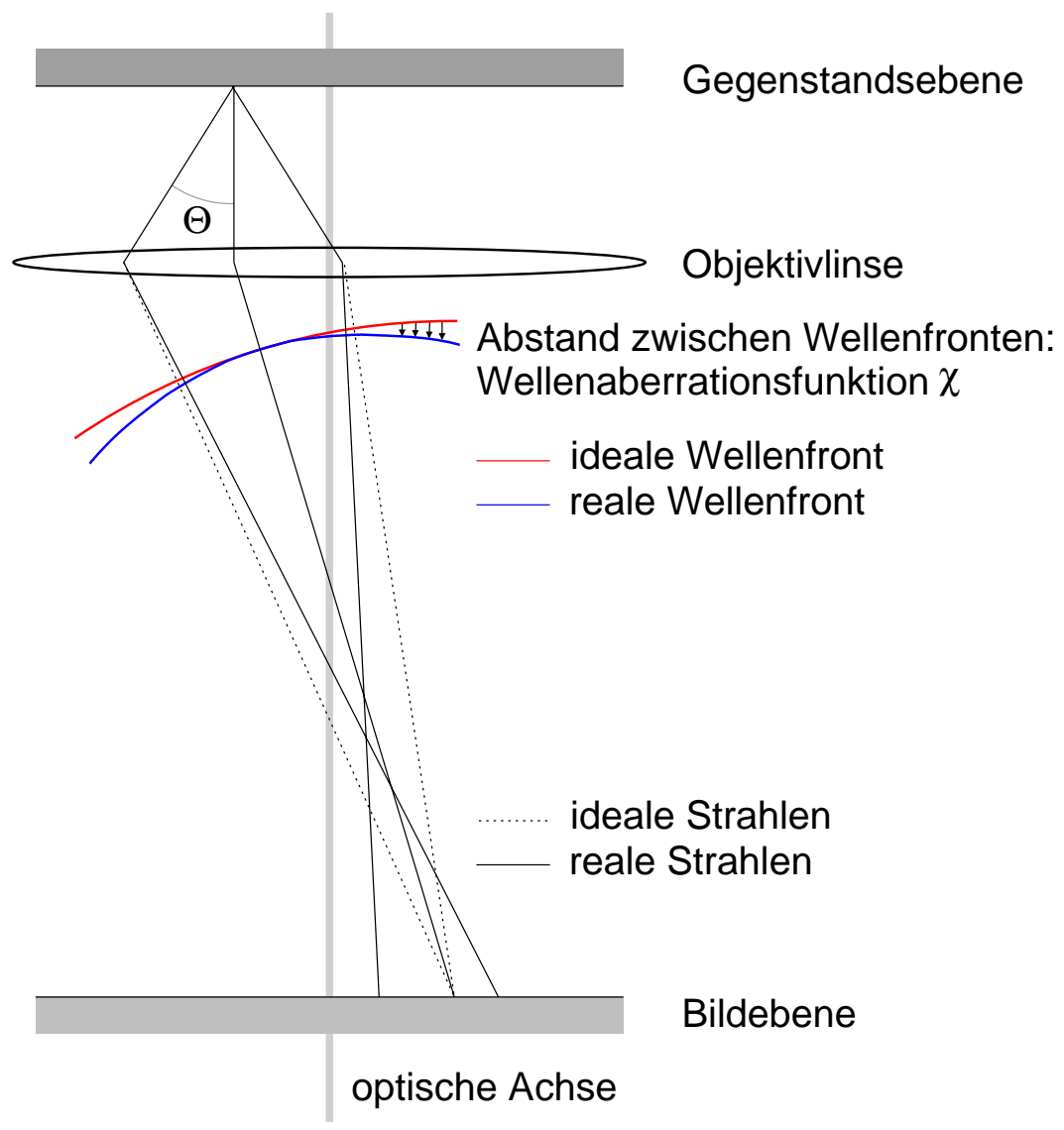

Abbildung 3.2: Bei einer idealen Abbildung ist die Wellenfront, die auf einen Bildpunkt in der Bildebene zuläuft, eine auf diesen Punkt zentrierte Kugelwelle. Als Wellenaberrationsfunktion $\chi(\boldsymbol{q})$ wird der Abstand zwischen dieser idealen und der realen Wellenfront in Abhängigkeit des Beugungswinkels $\sin \Theta \approx \Theta=\lambda \boldsymbol{q}$ definiert, wobei $\lambda$ die Wellenlänge der Elektronenwelle ist. 
optischen Achse [68]:

$$
\begin{aligned}
\chi\left(q_{x}, q_{y}\right)= & A_{1 x} q_{x}+A_{1 y} q_{y} \\
& +\frac{1}{2} \lambda D\left(q_{x}^{2}+q_{y}^{2}\right)+\frac{1}{2} \lambda\left[A_{2 x}\left(q_{x}^{2}-q_{y}^{2}\right)+A_{2 y}\left(2 q_{x} q_{y}\right)\right] \\
& +\lambda^{2}\left[B_{x} q_{x}\left(q_{x}^{2}+q_{y}^{2}\right)+B_{y} q_{y}\left(q_{x}^{2}+q_{y}^{2}\right)\right] \\
& +\frac{1}{3} \lambda^{2}\left[A_{3 x} q_{x}\left(q_{x}^{2}-3 q_{y}^{2}\right)+A_{3 y} q_{y}\left(3 q_{x}^{2}-q_{y}^{2}\right)\right] \\
& +\frac{1}{4} \lambda^{3} C_{s}\left(q_{x}^{2}+q_{y}^{2}\right)^{2} .
\end{aligned}
$$

Die Terme mit $\left(A_{1 x}, A_{1 y}\right)$ beschreiben eine Verschiebung des Bildes und können ohne Beschränkung der Allgemeinheit null gesetzt werden. Sie ändern sich im Falle einer Strahlverkippung aufgrund der anderen Linsenfehler (s. Abschnitt 3.4.2). Der Term mit $D$ beschreibt den Effekt der Defokussierung ${ }^{2}$.

Der 2-zählige und 3-zählige Astigmatismus findet sich in den Termen mit $\left(A_{2 x}, A_{2 y}\right)$ bzw. $\left(A_{3 x}, A_{3 y}\right)$ wieder. Als Astigmatismus wird der Effekt bezeichnet, dass Strahlen in Abhängigkeit von ihrem Azimuthalwinkel auf eine andere Bildebene abgebildet werden, d.h. eine unterschiedliche Brennweite haben. Der Astigmatismus lässt sich durch spezielle Multipollinsen innerhalb des Mikroskops korrigieren.

Die axiale Koma wird durch die Terme mit $\left(B_{x}, B_{y}\right)$ beschrieben. Eine ideale Linse bildet parallele Strahlen auf einen Punkt in der Brennebene ab. Die axiale Koma führt dazu, dass diese Bündelung der Strahlen asymmetrisch verschmiert. Durch die sphärische Aberration führt eine Strahlkippung (s. Abschnitt 3.4.2) zu einer Änderung der Größe der axialen Koma, so dass für eine bestimmte Strahlrichtung die Koma aufgehoben wird (Coma-Free-Alignment).

Der Term mit $C_{s}$ beschreibt die sphärische Aberration der Linse. Bei sphärischer Aberration werden Strahlen, je nach Winkel zur optischen Achse, auf verschiedene Bildebenen fokussiert. Wie in Abschnitt 3.1.2.2 deutlich wird, ist die sphärische Aberration bei Elektronenmikroskopen die das Punktauflösungsvermögen beschränkende Größe [70] (mit Ausnahme von Hochspannungsmikroskopen). Korrektoren für die sphärischen Aberration sind erst in den letzten Jahren kommerziell verfügbar geworden, da vorher die benötigte Stabilität und Genauigkeit der Abstimmung der Vielzahl an Multipol- und Transferlinsen, aus denen sie bestehen, technisch nicht erreichbar war [71].

Die nicht-rotationssymmetrischen Aberrationen, also diejenigen, die mit durch $x$ und $y$ indizierten Konstanten beschrieben werden, können auch durch ihren Betrag und ihren Azimuthalwinkel beschrieben werden. Ihr Betrag berechnet sich dabei immer entsprechend zu $K=\sqrt{K_{x}^{2}+K_{y}^{2}}$. In dieser Arbeit wird teilweise der Einfachheit halber nur ihr Betrag genannt.

Die Aberrationsfunktion, Gl. (3.1), lässt sich aufteilen in einen symmetrischen Anteil $\chi_{\mathrm{s}}(\boldsymbol{q})=$ $\chi_{s}(-\boldsymbol{q})$, der die symmetrischen Aberrationen enthält, d.h. die sphärische Aberration, den Defokus und den 2-zähligen Astigmatismus, sowie in einen antisymmetrischen Anteil $\chi_{\mathrm{a}}(\boldsymbol{q})=$

\footnotetext{
${ }^{2}$ Strenggenommen ist der Term mit $D$ kein Linsenfehler, sondern die Propagation der Welle zwischen der Rückseite der Probe und der fokussierten Ebene (s. Abb. 3.1), die gesondert berücksichtigt werden müsste. Da diese Propagation von einem zusätzlichen winkelabhängigen Phasenfaktor beschrieben wird, lässt sich der Defokus mathematisch analog zu den Linsenfehlern behandeln [69].
} 
$-\chi_{\mathrm{a}}(-\boldsymbol{q})$ mit den antisymmetrischen Aberrationen, d.h. die Bildverschiebung, den 3-zähligen Astigmatismus und die axiale Koma. Der symmetrische Anteil lässt sich unter bestimmten Bedingungen (z.B. für dünne amorphe Proben) direkt beobachten. Für die Messung des antisymmetrischen Anteils ist erheblich mehr Aufwand notwendig. In Abschnitt 3.4 werden Verfahren zur Messung beider Anteile erläutert.

\subsubsection{Bildentstehung}

In diesem Abschnitt wird erklärt, wie eine Abbildung aus der Objektwelle entsteht und welchen Einfluss die Aberrationsfunktion auf das Übertragungsverhalten des Mikroskops hat. Die Kenntnis dieser Bildentstehung ist notwendig, um die Funktionsweise der Defokusserienrekonstruktion zu erläutern. In speziellen Fällen kann die Bildentstehung als linearer Prozess beschrieben werden. Dies erlaubt unter anderem, die symmetrischen Anteile der Aberrationsfunktion aus einer einzelnen Abbildung zu bestimmen, was später zur Messung der Linsenfehler genutzt wird.

Zunächst wird im Folgenden der allgemeine Fall der Abbildung behandelt und dabei auf den komplexen Zusammenhang zwischen Abbildung und Objektwelle sowie die damit verbundenen Schwierigkeiten für eine Defokusserienrekonstruktion eingegangen.

Die quasikohärente Näherung erlaubt es, diesen Zusammenhang einfacher darzustellen, als die Kombination einer linearen Antwort des abbildenden Systems gefolgt von einer Betragsbildung. Dies wird im Abschnitt 3.1.2.2 näher erläutert. In diesem Zusammenhang lassen sich auch Größen wie Punktauflösung und Informationslimit definieren.

Für bestimmte Objekte bzw. Objektwellenfunktionen, wie in dem später vorgestellten Spezialfall des schwachen Phasenobjektes, lässt sich die Abbildung linearisieren. Die lineare Abbildung wird in Abschnitt 3.1.2.3 nochmals gesondert betrachtet.

\subsubsection{Allgemeine Bildentstehung}

Die hier gegebene Einführung folgt im Wesentlichen der Darstellung von Coene et al., die in [72] gegeben ist. Laut Ishizuka [73] lässt sich die Fouriertransformation

$$
\widetilde{I}(\boldsymbol{q})=\mathcal{F}\{I(\boldsymbol{r})\}=\int \mathrm{d} \boldsymbol{r}^{2} I(\boldsymbol{r}) \exp \{-\mathrm{i} 2 \pi \boldsymbol{q} \cdot \boldsymbol{r}\}
$$

der Intensitäten $I(r)$ in einer Abbildung schreiben als

$$
\widetilde{I}(\boldsymbol{q})=\int \mathrm{d} \boldsymbol{q}^{\prime 2} T\left(\boldsymbol{q}+\boldsymbol{q}^{\prime}, \boldsymbol{q}^{\prime}\right) \widetilde{\psi}\left(\boldsymbol{q}+\boldsymbol{q}^{\prime}\right) \widetilde{\psi}^{*}\left(\boldsymbol{q}^{\prime}\right),
$$

wobei $\widetilde{\psi}(\boldsymbol{q})=\mathcal{F}\{\psi(\boldsymbol{r})\}$ die Fouriertransformierte der Objektwellenfunktion $\psi(\boldsymbol{r})$, d.h. der Elektronenwellenfunktion auf der Rückseite der Probe, ist. Die Transmissionskreuzkoeffizienten (TCC)

$$
T\left(\boldsymbol{q}, \boldsymbol{q}^{\prime}\right)=p(\boldsymbol{q}) p^{*}\left(\boldsymbol{q}^{\prime}\right) E_{s}\left(\boldsymbol{q}, \boldsymbol{q}^{\prime}\right) E_{\Delta}\left(\boldsymbol{q}, \boldsymbol{q}^{\prime}\right)=T^{*}\left(\boldsymbol{q}^{\prime}, \boldsymbol{q}\right)
$$

beschreiben zum einen mittels $p(\boldsymbol{q})$ den Einfluss der Objektivlinse, somit die Veränderung der Abbildung durch die Linsenfehler, und zum anderen die Wirkung der eingeschränkten Kohärenz des Elektronenstrahls in Form der Dämpfungsfunktionen $E_{s}$ und $E_{\Delta}$. 
Die linsenfehlerbeschreibende (reelle) Aberrationsfunktion $\chi(\boldsymbol{q})$ (s. Abschitt 3.1.1) führt zu einem Phasenfaktor:

$$
p(\boldsymbol{q})=\exp \{-2 \pi \mathrm{i} \chi(\boldsymbol{q})\}
$$

Die räumliche Kohärenz des Strahls wird durch die endliche Größe der Elektronenquelle eingeschränkt. Dies wird durch den Faktor

$$
E_{s}\left(\boldsymbol{q}, \boldsymbol{q}^{\prime}\right)=\exp \left\{-\left(\frac{\pi \alpha}{\lambda}\right)^{2}\left[\left.\nabla \chi\right|_{\boldsymbol{q}}-\left.\nabla \chi\right|_{\boldsymbol{q}^{\prime}}\right]^{2}\right\}=E_{s}\left(\boldsymbol{q}^{\prime}, \boldsymbol{q}\right)
$$

berücksichtigt, wobei $\alpha$ der Halbwinkel der Strahlkonvergenz des Mikroskops ist und $\lambda$ die Wellenlänge der Elektronen ( $\nabla$ ist der Differentialoperator bzgl. $\boldsymbol{q}$ ). Mikroskope mit Feldemissionsquellen (FEG) haben eine hohe räumliche Kohärenz, d.h. einen sehr kleinen Semikonvergenzwinkel $\alpha$ (z.B. $\alpha=0.2 \mathrm{mrad}$ bei dem in dieser Arbeit verwendeten Mikroskop). Dies erlaubt eine Faktorisierung

$$
E_{s}\left(\boldsymbol{q}, \boldsymbol{q}^{\prime}\right) \approx E_{s}(\boldsymbol{q}) E_{s}\left(\boldsymbol{q}^{\prime}\right) \operatorname{mit} E_{s}(\boldsymbol{q})=E_{s}(\boldsymbol{q}, 0) .
$$

Zeitliche Schwankungen in der Hochspannungsversorgung des Beschleunigers, $\Delta V$, in den Linsenströmen, $\Delta J$, sowie die thermisch bedingte Energiebreite $\Delta E$ der Emission aus der Elektronenquelle führen zu einer eingeschränkten zeitlichen Kohärenz des Elektronenstrahls. Dieser Effekt entspricht einer Streuung des Defokus um den eigentlichen Wert (chromatische Streuung), da jedes Elektron eine leicht unterschiedliche Ablenkung durch die Objektivlinse verspürt. Laut Ishizuka [73] lässt sich dies durch die Einhüllende

$$
E_{\Delta}\left(\boldsymbol{q}, \boldsymbol{q}^{\prime}\right)=\exp \left\{-2(\pi \Delta)^{2}\left[\left.\frac{\partial \chi}{\partial D}\right|_{\boldsymbol{q}}-\left.\frac{\partial \chi}{\partial D}\right|_{\boldsymbol{q}^{\prime}}\right]^{2}\right\}=E_{\Delta}\left(\boldsymbol{q}^{\prime}, \boldsymbol{q}\right)
$$

mit $\Delta$ als der Breite der Defokusstreuung beschreiben, die sich aus dem chromatischen Linsenfehler $C_{c}$ der Objektivlinse und den relativen Schwankungen zusammensetzt:

$$
\Delta=C_{c} \sqrt{\left(\frac{\Delta V}{V}\right)^{2}+4\left(\frac{\Delta J}{J}\right)^{2}+\left(\frac{\Delta E}{V}\right)^{2}} .
$$

Als quasikohärente Näherung wird eine Faktorisierung dieser Einhüllenden bezeichnet:

$$
E_{\Delta}\left(\boldsymbol{q}, \boldsymbol{q}^{\prime}\right) \approx E_{\Delta}(\boldsymbol{q}) E_{\Delta}\left(\boldsymbol{q}^{\prime}\right) \operatorname{mit} E_{\Delta}(\boldsymbol{q})=E_{\Delta}(\boldsymbol{q}, 0) .
$$

Diese Näherung führt zwar für nicht-lineare Abbildungen zu nicht vernachlässigbaren Fehlern, liefert aber im folgenden Abschnitt weitere Einsichten.

Die Abbildungsgleichung (3.2) zeigt, dass Beiträge der Wellenfunktion $\widetilde{\psi}(\boldsymbol{q})$ und ihres konjugierten Gegenparts $\widetilde{\psi}^{*}(-\boldsymbol{q})$ stark miteinander verbunden sind: Für zwei Raumfrequenzen $\boldsymbol{q}$ und $\boldsymbol{q}^{\prime}$ tragen sowohl das Produkt $\widetilde{\psi}(\boldsymbol{q}) \widetilde{\psi}^{*}\left(\boldsymbol{q}^{\prime}\right)$ als auch das Produkt $\widetilde{\psi}^{*}(-\boldsymbol{q}) \widetilde{\psi}\left(-\boldsymbol{q}^{\prime}\right)$ zu gleichen Anteilen, allerdings mit unterschiedlichen Phasen, zur Bildintensität $\widetilde{I}\left(\boldsymbol{q}-\boldsymbol{q}^{\prime}\right)$ bei. Die Trennung dieser beiden Beiträge ist die Hauptschwierigkeit bei der Defokusserienrekonstruktion. Da sich die Phasenfaktoren mit dem Defokus ändern, werden bei der Defokusserienrekonstruktion mehrere Abbildungen mit unterschiedlichen Defokuswerten benutzt, um diese Trennung zu ermöglichen. 


\subsubsection{Quasikohärente Näherung}

Mit der Kontrasttransferfunktion (CTF)

$$
\gamma(\boldsymbol{q})=p(\boldsymbol{q}) E_{s}(\boldsymbol{q}) E_{\Delta}(\boldsymbol{q})
$$

lassen sich die Transmissionskreuzkoeffizienten innerhalb der quasikohärenten Näherung (s. letzter Abschnitt) faktorisieren:

$$
T\left(\boldsymbol{q}, \boldsymbol{q}^{\prime}\right) \approx \gamma(\boldsymbol{q}) \gamma^{*}\left(\boldsymbol{q}^{\prime}\right) .
$$

Wird Gl. (3.7) in die Abbildungsgleichung (3.2) eingesetzt, so wird die Abbildung $\widetilde{I}(\boldsymbol{q})$ eine Faltung der Bildwelle

$$
\widetilde{\psi}_{B}(\boldsymbol{q})=\gamma(\boldsymbol{q}) \widetilde{\psi}(\boldsymbol{q})
$$

mit sich selbst.

Dies ergibt im Zusammenhang mit dem Faltungstheorem eine einfache Deutung der quasikohärenten Abbildung im Realraum. In der Bildwelle

$$
\psi_{B}(r)=\mathcal{F}^{-1}\left\{\widetilde{\psi}_{B}(\boldsymbol{q})\right\}
$$

führen die Linsenfehler und die eingeschränkte Kohärenz zu einer optischen Delokalisierung der Objektwelle $\psi(\boldsymbol{r})$. Diese Delokalisierung kann innerhalb einer Linearen-Antwort-Theorie durch eine Faltung mit der Punktverschmierungsfunktion, d.h. der Fouriertransformation der $\mathrm{CTF}$, beschrieben werden:

$$
\psi_{B}(\boldsymbol{r})=\mathcal{F}^{-1}\{\gamma(\boldsymbol{q})\} \otimes \psi(\boldsymbol{r}) .
$$

In der Bildebene wird die Intensität dieser Bildwelle gemessen:

$$
I(\boldsymbol{r})=\left\|\psi_{B}(\boldsymbol{r})\right\|^{2} .
$$

Wird die komplexe CTF als Antwort des optischen Systems interpretiert, so beschreibt ihre Amplitude, wie stark eine Raumfrequenz übertragen wird, während die Phase angibt, mit welcher Phasenlage die Bildinformationen dieser Frequenz übertragen werden. In Abb. 3.3 sind sowohl der Imaginärteil der CTF für den Scherzerfokus (s.u.), als auch die beiden einhüllenden Dämpfungsfunktionen gezeigt. Der Vergleich der CTF mit den beiden Einhüllenden macht deutlich, dass FEG-Mikroskope eine hohe räumliche Kohärenz besitzen und daher primär durch die eingeschränkte zeitliche Kohärenz limitiert sind. Das Informationslimit $q_{\text {info }}$ des Mikroskops ist die höchste übertragene Raumfrequenz. Es wird über die zeitliche Einhüllende definiert: $E_{\Delta}\left(q_{\text {info }}\right)=e^{-2}$. Für das in dieser Arbeit verwendete Mikroskop ist $q_{\text {info }}=8 \mathrm{~nm}^{-1}$.

Der Scherzerfokus ${ }^{3}$

$$
D_{\mathrm{Sch}}=\sqrt{\frac{3}{2} C_{\mathrm{s}} \lambda}
$$

zeichnet sich dadurch aus, dass für ein breites Frequenzband (hier von etwa $1.5-5.0 \mathrm{~nm}^{-1}$ ) die Abbildung mit einer Phasenverschiebung um $\pi / 2$ übertragen wird, was durch eine weitgehende Kompensation der sphärischen Aberration durch den Defokus für diesen Frequenzbereich

\footnotetext{
${ }^{3}$ Die Definition des Scherzerfokus ist in der Literatur uneinheitlich. Die obige Definition stammt von Spence [69]. Williams und Carter definieren den $D_{\text {Sch }}$ mit einem Faktor $\frac{4}{3}$ anstatt $\frac{3}{2}$ [57]. Alexander [74] bezeichnet den obigen Defokus als optimalen Fokus und definiert den Scherzerfokus als $\sqrt{C_{s} \lambda}$.
} 


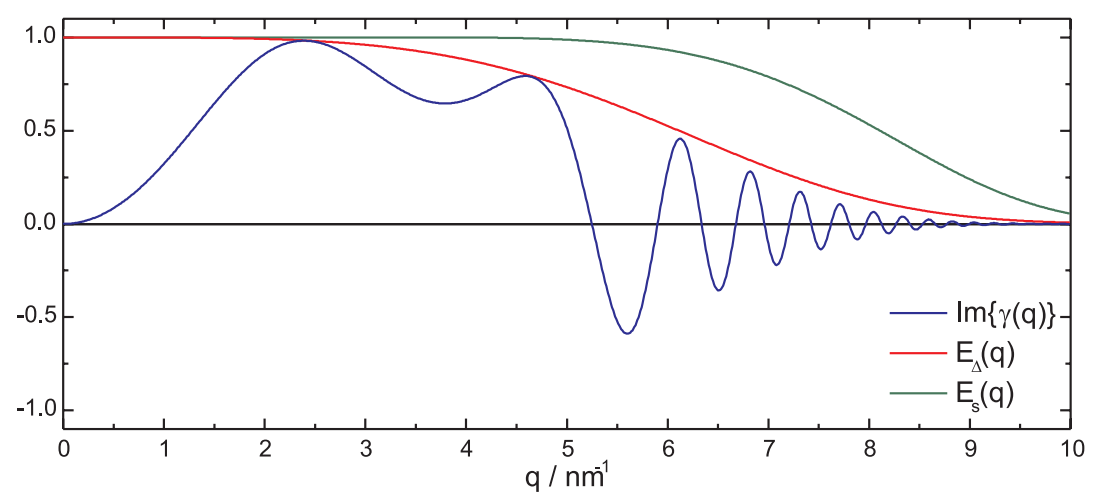

Abbildung 3.3: Imaginärteil der Kontrasttransferfunktion beim Scherzerfokus $\left(D_{\text {Sch }}=-43.3 \mathrm{~nm}, C_{s}=\right.$ $0.5 \mathrm{~mm}$, keine weiteren Aberrationen) und die Dämpfungsfunktionen ( $\Delta=4 \mathrm{~nm}, \alpha=0.2 \mathrm{~nm})$ für ein 200 $\mathrm{kV}$ Mikroskop $(\lambda=2.51 \mathrm{pm})$. Für ein breites Frequenzband von etwa $1.5-5.0 \mathrm{~nm}^{-1}$ werden die Bildanteile mit nahezu gleicher Phasenlage übertragen, während es bei der Übertragung höherer Frequenzen zu einem starkem Oszillieren der Phase kommt. Die eingeschränkte zeitliche Kohärenz, berücksichtigt durch die Einhüllende $E_{\Delta}$, dämpft die Übertragung von hochfrequenten Bildanteilen weit stärker als die räumliche Kohärenz, berücksichtigt durch die Einhüllende $E_{s}$.

erreicht wird. Die Phasenschiebung von $\pi / 2$ gegenüber dem Nullstrahl ist dabei nötig, um Phasenobjekte (s. Abschnitt 3.3.1) in einer mit dem Zernike-Phasenmikroskop vergleichbaren Weise sichtbar zu machen.

Das Punktauflösungvermögen $d_{p}$ wird über den 1. Nulldurchgang der CTF im Scherzerfokus definiert [69], da die Linsenfehler, d.h. im wesentlichen $C_{\mathrm{s}}$, bei höheren Raumfrequenzen zu wechselnden Phasenlagen und zu starken Gradienten des Phasentransfers führen. Bei dem verwendeten Mikroskop ist $d_{p}=0.19 \mathrm{~nm}\left(1\right.$. Nulldurchgang bei $\left.5.25 \mathrm{~nm}^{-1}\right)$. Die Gradienten des Phasentransfers bewirken die optische Delokalisierung, die die Interpretation der Abbildung erschwert, so dass entweder die hohen Raumfrequenzen mit einer Objektivblende ausgeblendet werden müssen, oder Vergleiche mit Simulationen notwendig werden.

Ist das Mikroskop mit Korrektoren für die sphärische Aberration ausgestattet, so wird es möglich, den Phasentransfer für den gesamten Frequenzbereich bis zum Informationslimit optimal einzustellen, d.h. die Delokalisierung zu minimieren. Bei der Objektwellenrekonstruktion wird die komplexe Wellenfunktion verfügbar, so dass die optische Delokalisierung vollständig behoben werden kann, da auch der Phasenkontrast nicht mehr durch die Transferfunktion sichtbar gemacht werden muss.

\subsubsection{Lineare Abbildung}

In vielen Fällen (wie z.B. beim schwachen Phasenobjekt) ist der Nullstrahl stärker als die abgebeugten Strahlen:

$$
\|\widetilde{\psi}(0)\| \gg\|\widetilde{\psi}(\boldsymbol{q} \neq 0)\|
$$

Dies erlaubt, die Abbildungsgleichung (3.2) bzgl. der abgebeugten Strahlen zu linearisieren:

$$
\widetilde{I}(\boldsymbol{q} \neq 0) \approx \widetilde{\psi}(0) \widetilde{\psi}^{*}(-\boldsymbol{q}) \gamma^{*}(-\boldsymbol{q})+\widetilde{\psi}^{*}(0) \widetilde{\psi}(\boldsymbol{q}) \gamma(\boldsymbol{q}),
$$


und $\widetilde{I}(0) \approx\|\widetilde{\psi}(0)\|^{2}$. Dabei wurde ausgenutzt, dass $\gamma(0)=1$ ist und daher

$$
T(\boldsymbol{q}, 0)=T^{*}(0, \boldsymbol{q})=\gamma(\boldsymbol{q})
$$

gilt. In diesem linearen Fall wird die quasikohärente Näherung exakt, da keine Faktorisierung der TCC vorgenommen werden muss.

Die Defokusserienrekonstruktion ist in diesem Spezialfall einfacher, da eine analytische Umkehrung von Gl. (3.9) für bereits zwei Aufnahmen mit unterschiedlichem Defokus möglich ist (außer für Frequenzen $\boldsymbol{q}$, bei denen $\gamma(\boldsymbol{q})$ für beide Abbildungen identisch ist).

\subsubsection{Einfluss der CCD-Kamera}

Ein weiterer Einfluss auf die aufgezeichneten Abbildungen wurde bis jetzt nicht beachtet: der Einfluss des Aufzeichnungsmediums selbst. Die Abbildungen, die zur Defokusserienrekonstruktion verwendet wurden, sind mit einer CCD-Kamera aufgezeichnet worden. Ausführlichere Beschreibungen der Einflüsse einer CCD-Kamera sind bei Zuo [75] und Meyer [76] zu finden.

Der CCD-Chip wird dabei nicht selbst dem Elektronenstrahl ausgesetzt, wie es etwa bei einer Photoplatte der Fall wäre. Die Elektronen erzeugen stattdessen in einem Szintillator Photonen und diese Photonen werden durch eine Glasfaseroptik auf den CCD-Chip abgebildet. Da im Szintillator die Kohärenz der Elektronenwelle sehr schnell zerstört wird, kann diese Messung durchaus im Einelektronenbild beschrieben werden. Die Bildwellenfunktion $\psi_{B}(\boldsymbol{r})$ gibt somit nur die Wahrscheinlichkeit $I(\boldsymbol{r})=\left\|\psi_{B}(\boldsymbol{r})\right\|^{2}$ an, dass ein Elektron an einem bestimmten Ort gemessen wird, d.h. in den Szintillator trifft.

Weitere Streuung der Primärelektronen und der sekundären Photonen im Szintillator führt dazu, dass für ein einzelnes Elektron nicht nur ein Signal in einem einzelnen Pixel gemessen wird, sondern auch in den umgebenen Pixeln. Anders gesehen führt dies für die aufgezeichnete Abbildung

$$
\widetilde{I}^{\prime}(\boldsymbol{q})=M(q) \widetilde{I}(\boldsymbol{q})
$$

zu einer Dämpfung hoher Raumfrequenzen, die durch die radialsymmetrische Modulationstransferfunktion $M(q)$ beschrieben werden kann. Weitere Einflüsse auf die aufgezeichnete Abbildung sind ortsabhängige Verstärkungsfaktoren und ein Hintergrundrauschen der Kamera selbst. Beide werden bereits innerhalb der Aufzeichnungssoftware korrigiert.

Die genaue Form von $M(q)$ für die verwendete Kamera wurde gesondert vermessen [77]. Diese Messung wurde in jüngerer Zeit wiederholt, um Alterungseffekte auszuschließen. Die Modulationstransferfunktion wird bei der Defokusserienrekonstruktion mit berücksichtigt.

\subsection{Prinzip der Defokusserienrekonstruktion}

Bei der Defokusserienrekonstruktion wird die Objektwelle $\psi$ aus einer Reihe von $N$ (typischerweise 10-20) Abbildungen $I_{n}$ bestimmt, die bei unterschiedlichen Abbildungsbedingungen aufgenommen wurden. Zwischen den Aufnahmen wird dabei der Defokus $D$ verändert. Prinzipiell ließen sich auch andere Parameter ändern, z.B. die Strahlverkippung. 
In diesem Abschnitt soll das Prinzip zweier Ansätze zur Defokusserienrekonstruktion erläutert werden. Der erste Ansatz basiert auf linearen Filtern. Die Filter nutzen die Umkehrbarkeit der linearen Abbildungsgleichung (3.9) aus, um nur die linearen Anteile aus den Abbildungen herauszufiltern, d.h. bei der Rekonstruktion die nicht-linearen Anteile zu unterdrücken. Die hier vorgestellte Paraboloidmethode gehört zu den Methoden, die diesen Ansatz verfolgen. Der andere Ansatz basiert auf einem Maximum-Likelihood-Formalismus. Er berücksichtigt sowohl die linearen als auch die nicht-linearen Anteile in den Abbildungen bei der Rekonstruktion der Objektwelle. Diejenigen Größen, die sich über die Serie ändern, werden im Folgenden mit $n$ indiziert.

Die Paraboloidmethode wird ausführlich in [78] erläutert, ihr Prinzip soll hier stellvertretend für die Filter-Methoden vorgestellt werden. Werden die Effekte der eingeschränkten Kohärenz vernachlässigt, und sei der Einfachheithalber der Nullstrahl $\psi(0)=1$, so gilt für die lineare Abbildung:

$$
\widetilde{I}(\boldsymbol{q}, D)=\delta(\boldsymbol{q})+\widetilde{\psi}_{B}(\boldsymbol{q}) \exp \left(-\pi \mathrm{i} \lambda D q^{2}\right)+\widetilde{\psi}_{B}^{*}(-\boldsymbol{q}) \exp \left(\pi \mathrm{i} \lambda D q^{2}\right)
$$

wobei $\psi_{B}$ die Bildwelle im Gaußschen Fokus $(D=0)$ ist. Eine Fouriertransformation dieser Gleichung bzgl. des Defokus ergibt mit $\zeta$ als zu $D$ adjungierter Variable

$$
\widetilde{I}(\boldsymbol{q}, \zeta) \propto \delta(\boldsymbol{q}, \zeta)+\widetilde{\psi}_{B}(\boldsymbol{q}) \delta\left(\zeta+\frac{1}{2} \lambda q^{2}\right)+\widetilde{\psi}_{B}^{*}(-\boldsymbol{q}) \delta\left(\zeta-\frac{1}{2} \lambda q^{2}\right)
$$

Das bedeutet, dass sich die Bildwelle im reziproken Raum auf einem Paraboloiden mit $\zeta=$ $-\frac{1}{2} \lambda q^{2}$ bzw. die konjugierte Welle auf dem an der Ebene $\zeta=0$ gespiegelten Paraboloiden befindet. Diese Paraboloiden entsprechen dabei der Ewaldkugel [79]. Eine Rekonstruktion ist nun prinzipiell möglich, wenn für jede Raumfrequenz $\boldsymbol{q}$ der zu $\widetilde{\psi}_{B}(\boldsymbol{q})$ gehörige Anteil aus dem entsprechenden Paraboloiden herausgefiltert wird:

$$
\widetilde{\psi}_{B}(\boldsymbol{q}) \propto \sum_{n=1}^{N} \widetilde{I}_{n}(\boldsymbol{q}) \exp \left(\pi \mathrm{i} \lambda D_{n} q^{2}\right) .
$$

Dieser Ansatz lässt sich erweitern, so dass die konjugierte Welle, die Effekte der partiellen Kohärenz und die nichtlinearen Beiträge sowie Rauschen berücksichtigt werden.

Die Paraboloidmethode ist eine der Methoden, die auf linearen Filtern basieren. Bei diesen Methoden werden die Abbildungen mit einer Filterfunktion multipliziert, und es wird über die Serie summiert, wie in Gl. (3.10) in einfacher Form zu sehen ist. Die Form der Filterfunktionen wird dabei aus der linearen Abbildungsgleichung abgeleitet. Einen Überblick über die verschiedenen derartigen Methoden, die erste wurde bereits 1968 von Schiske vorgeschlagen, und ihre engen Zusammenhänge ist bei Saxton [79] zu finden. Der Nachteil dieser Methoden ist, dass sie nur für die lineare Abbildung exakt sind und daher versuchen, nur die linearen Anteile aus den Aufnahmen herauszufiltern, während die nichtlinearen Anteile als Störung betrachtet werden.

Der andere Ansatz zur Defokusserienrekonstruktion basiert auf einem Maximum-Likelihood (MAL) Formalismus. Die hier gegebene Einführung skizziert die Methode von Coene et al. [72]. Der ursprüngliche Ansatz stammt von Kirkland, der auch eine Herleitung für diesen Formalismus gibt [80]. Das Funktional

$$
S_{\psi}^{2}=\sum_{n=1}^{N} \int \mathrm{d} \boldsymbol{q}^{2}\left[\widetilde{I}_{n}^{\exp }(\boldsymbol{q})-\widetilde{I}_{n, \psi}^{\text {theo }}(\boldsymbol{q})\right]^{2}
$$


wird hier minimiert, dabei sind $\widetilde{I}_{n}^{\exp }$ die experimentellen Aufnahmen und $\widetilde{I}_{n, \psi}^{\text {theo }}$ die für eine Objektwelle $\psi$ bei gegebenen Abbildungsbedingungen nach Gl. (3.2) zu erwartenden Abbildungen. Diese Minimierung wird durch ein iteratives Verfahren erreicht, bei dem für eine Objektwelle $\psi_{j}$ aus dem $j$-ten Schritt die Objektwelle $\psi_{j+1}$ für den nächsten Schritt bestimmt wird

$$
\widetilde{\psi}_{j+1}(\boldsymbol{q})=\widetilde{\psi}_{j}(\boldsymbol{q})-\beta_{j} g_{j}(\boldsymbol{q}) .
$$

Die Änderung zwischen Schritten besteht dabei aus einer Schrittweite $\beta_{j}$ und einer Richtung $g_{j}(\boldsymbol{q})$, die z.B. der Gradient des Fehlerfunktionals bzgl. der Wellenfunktion sein kann (,Steepest Descent-MAL"; SD-MAL):

$$
g_{j}(\boldsymbol{q})=\left.\frac{\partial S^{2}}{\partial \widetilde{\psi}(\boldsymbol{q})}\right|_{\widetilde{\psi}_{j}} .
$$

Mehr zur Wahl der Schrittweite sowie andere Möglichkeiten für die Schrittrichtung sind bei Coene et al. zu finden [72]. Das Abbruchkriterium für die Iteration ist die Konvergenz des Funktionals $S_{\psi}^{2}$.

Im Gegensatz zu den Filter-Methoden, versucht dieser Ansatz nicht, die Abbildung selbst umzukehren. Er sucht stattdessen diejenige Wellenfunktion, die bei gegebenen experimentellen Aufnahmen die wahrscheinlichste ist. Dieser Ansatz ermöglicht die explizite Berücksichtigung der nichtlinearen Bildanteile und auch die Berücksichtigung der Kreuzterme (3.3), die durch die partielle Kohärenz entstehen. Um die Konvergenz zu beschleunigen, wird üblicherweise als Startwert $\widetilde{\psi}_{j=0}$ eine Rekonstruktion aus einer der oben vorgestellten Filtermethoden verwendet.

Gegenüber anderen holographischen Methoden, wie der Off-Axis-Holographie, hat die Defokusserienrekonstruktion den Nachteil, dass die Objektwellen für tiefe Raumfrequenzen $\left(<1.5 \mathrm{~nm}^{-1}\right.$ für typische Bedingungen) nicht vollständig rekonstruiert werden können: Zum einen ändert sich die CTF für diese Frequenzen nur schwach mit dem Defokus, so dass die Rekonstruktionen hier im Wesentlichen nur über die Abbildungen mitteln. Zum anderen sind in den Abbildungen bei diesen Frequenzen hauptsächlich die Amplituden-Anteile der Objektwelle zu finden, da die Phasenschiebung der CTF gegenüber des Nullstrahls hier sehr klein ist, und so keine Phasenanteile übertragen werden. Mit den vorgestellten Ansätzen wird also bei tiefen Frequenzen im Wesentlichen nur die Amplitude der Objektwelle rekonstruiert.

\subsection{Streuung im Objekt}

Die Näherung des schwachen Phasenobjektes erlaubt es, einen einfachen Zusammenhang zwischen der Struktur der Probe, genauer ihrem elektrostatischem Potential, und der Objektwelle herzustellen (Abschnitt 3.3.1), jedoch ist die Gültigkeit dieser Näherung auf dünne Proben begrenzt. Für dickere Proben müssen die Effekte der dynamischen Vielfachstreuung und die inelastischen Streuprozesse berücksichtigt werden.

Um trotzdem die Objektwelle mit der Struktur der Probe in Zusammenhang zu bringen, wird die Welle aus einem Strukturmodell mithilfe von Simulationen berechnet. Diese berechnete Welle kann anschließend mit der experimentell ermittelten Objektwelle verglichen werden. Die beiden üblichen Simulationsmodelle, Multislice- und Blochwellen-Rechnungen, beschreiben nur die elastisch gestreuten Anteile der Welle innerhalb der kohärenten Vielfachstreuung. 
Auftretende inelastische Streuprozesse und Streuung an Phononen zerstören diese Kohärenz. Daher werden sie in diesen Simulationen nur als Absorption, d.h. als Verringerung der Intensität, berücksichtigt.

Dass diese inelastischen und inkohärenten Prozesse einen nicht zu vernachlässigen Einfluss auf die Objektwelle haben, zeigen die gefundenen Abweichungen zwischen Simulationen und experimentellen Abbildungen, wie in Abschnitt 3.3.5 erläutert wird. Auch bei der Simulation der ALCHEMI-Messungen (s. Abschnitt 7.4) sind diese Prozesse zu berücksichtigen. Daher werden sie im Folgenden kurz angesprochen.

Für eine ausführlichere Besprechung der Streuprozesse sei auf die Literatur verwiesen [57, 64, $81,82]$.

\subsubsection{Starkes und schwaches Phasenobjekt}

Die Näherung des Phasenobjektes erlaubt es, die Streuung einer Elektronenwelle in speziellen Objekten, wie dünne amorphe oder schwach streuende Proben, einfach zu modellieren. Das schwache Phasenobjekt erfüllt dabei zusätzlich noch die Bedingungen der linearen Abbildung (s. Abschnitt 3.1.2.3). Dies ermöglicht eine einfache Interpretation der elektronenmikroskopischen Abbildungen. Desweiteren zeigt diese Näherung, wie es mit Hilfe von dünnen amorphen Objekten möglich ist, die symmetrischen Anteile der Aberrationsfunktion zu messen (s. auch Abschnitt 3.4.1).

Passiert eine ebene Elektronenwelle mit der Wellenfront $\psi_{0}(\boldsymbol{r})$ eine sehr dünne oder eine schwach streuende Probe, so wird die Phase der Welle in der Probe im Vergleich zu einer Welle im Vakuum nachlaufen. Das mittlere Probenpotential wirkt anziehend für Elektronen, daher haben Elektronen in der Probe eine höhere kinetische Energie als im Vakuum, was zu einer kürzeren Wellenlänge und somit zu einer Differenz in der Phase führt. Daher kann die Probe selbst als Phasenobjekt angesehen werden (Näherung des starken Phasenobjektes). Diese Näherung vernachlässigt die Wellenpropagation in der Probe, Mehrfachstreuung sowie Absorption. Sie entspricht der 1. Bornschen Näherung, beschreibt also die kinematische Streuung der Elektronen.

Für die Objektwelle $\psi(\boldsymbol{r})$ bedeutet dies

$$
\psi(\boldsymbol{r})=\psi_{0}(\boldsymbol{r}) \exp [\mathrm{i} \phi(\boldsymbol{r})] \operatorname{mit} \phi(\boldsymbol{r})=\pi \sigma V_{Z}(\boldsymbol{r})
$$

mit dem reellen Phasenschub $\phi(r)$, der sich aus dem über die Probendicke entlang des Strahls integrierten Potential $V_{Z}(\boldsymbol{r})$ und einer Wechselwirkungskonstante $\sigma$ berechnet [69]. Für schwach streuende Proben ist dieser Phasenschub klein, d.h. $\phi \ll 1$, so dass sich der entsprechende Phasenfaktor linearisieren lässt, was zur Näherung des schwachen Phasenobjektes führt:

$$
\psi(\boldsymbol{r}) \approx \psi_{0}(\boldsymbol{r})[1+\mathrm{i} \phi(\boldsymbol{r})], \text { für } \phi \ll 1
$$

Der Einfachheit halber seien $\psi_{0}(\boldsymbol{r})$ und $\phi(\boldsymbol{r})$ so gewählt, dass $\psi_{0}(\boldsymbol{r})=1$ und $\langle\phi(\boldsymbol{r})\rangle=0$ gilt. Die Fouriertransformierte der Objektwelle ist in diesem Fall

$$
\widetilde{\psi}(\boldsymbol{q})=\delta(\boldsymbol{q})+\mathrm{i} \widetilde{\phi}(\boldsymbol{q}) \operatorname{mit} \widetilde{\phi}(\boldsymbol{q})=\mathcal{F}\{\phi(\boldsymbol{r})\}
$$


Da die abgebeugten Anteile $\widetilde{\psi}(\boldsymbol{q} \neq 0)$ klein sind, lässt sich die Abbildung dieser Objektwelle linear beschreiben. Einsetzen in Gleichung (3.9) ergibt für den Betrag der Abbildung

$$
\|\widetilde{I}(\boldsymbol{q})\|=\delta(\boldsymbol{q})+2\|\widetilde{\phi}(\boldsymbol{q})\|\left|E_{s}(q) E_{\Delta}(q) \sin \left(-2 \pi \chi_{\mathrm{s}}(\boldsymbol{q})\right)\right| .
$$

Die Terme im letzten Betrag in der obigen Gleichung entsprechen dem Imaginärteil der symmetrisierten Kontrasttransferfunktion (s. Abb. 3.3).

In amorphen Proben ist $\|\widetilde{\phi}(\boldsymbol{q})\|$ eine endliche, zu den hohen Raumfrequenzen hin abfallende Größe. Die Dämpfungsfunktionen fallen ebenfalls zum Informationslimit hin ab. Der SinusTerm ist der einzige, der sich schnell mit $\boldsymbol{q}$ ändert (die Oszillationen von $\|\widetilde{\phi}(\boldsymbol{q})\|$ sind dagegen für die meisten Bedingungen langsam). Daher lassen sich die Nulldurchgänge des Sinus-Terms in $\|\widetilde{I}(\boldsymbol{q})\|$ deutlich sehen, wie z.B. in Abb. $3.4 \mathrm{zu}$ erkennen ist.

\subsubsection{Elastische kohärente Streuung}

Die elastische Streuung der Elektronen geschieht am elektrostatischen Potential $V(\boldsymbol{r})$ der Probe. Dieses Streupotential wird dabei in den Simulationen als Superposition der atomaren Streupotentiale der freien Atome (Atomformfaktoren) am Ort der Atome innerhalb der Einheits- bzw. Super-Zelle modelliert. Da andere Streuprozesse innerhalb von herkömmlichen Simulationen nicht berücksichtigt werden, wird dem reellen Streupotentials $V(r)$ ein imaginärer Anteil $V^{\prime}(r)$ hinzugefügt:

$$
U(\boldsymbol{r})=\sigma V(\boldsymbol{r})+\mathrm{i} \sigma V^{\prime}(\boldsymbol{r})
$$

Der imaginäre Anteil (auch Absorptionspotential oder optisches Potential genannt) verringert die Intensität der Elektronen innerhalb der kohärenten Vielfachstreuung, da die inelastisch bzw. inkohärent gestreuten Elektronen nicht mehr zu ihr beitragen. In experimentellen Abbildungen tragen die ,absorbierten“ Elektronen immer noch zu einem diffusen Hintergrund bei, der allerdings nicht mehr innerhalb der Simulationen beschrieben wird.

Meist wird der imaginäre Anteil proportional zum reellen Anteil gewählt

$$
V^{\prime}(r)=\alpha V(r)
$$

mit einer Absorptionskonstante $\alpha$ im Bereich $<0.1$.

\subsubsection{Thermisch diffuse Streuung}

Die thermisch diffuse Streuung (TDS) oder auch Phononenstreuung kommt durch die thermische Vibration der Atome um ihre Gleichgewichtslage zustande. Da die einzelnen Atome als quantenmechanischer Oszillator angesehen werden können, tritt diese Vibration auch noch am absoluten Temperaturnullpunkt (wenn auch schwächer) auf. Die durch TDS gestreuten Elektronen tragen zu einem diffusen Hintergrund des Beugungsbildes bei, der nur noch schwache Bragg-Streuung erfährt (Kikuchi-Linien). Für kristalline Proben bedeutet dies, dass auch Elektronen unter Winkeln, die zwischen den Bragg-Winkeln liegen, die Probe verlassen, was z.B. von Blochwellenrechnungen nicht mehr berücksichtigt wird. 
Der Einfluss dieser Streuung in den Simulationen wird auf zwei Weisen berücksichtigt: Die zeitlich gemittelte Verschmierung der einzelnen Atome um ihre nominelle Lage wird durch einen Debye-Waller-Faktor beschrieben, wie dies auch in der Röntgenstreuung üblich ist. Der andere zu beschreibende Effekt besteht darin, dass die Elektronen bei dem Streuvorgang Phononen anregen und vernichten. Dabei werden nur kleine Energien $(<0.1 \mathrm{eV})$ übertragen, so dass der Energieübertrag vernachlässigt werden kann (quasi-elastische Streuung). Allerdings ändert sich auch der quantenmechanische Zustand des Objektes, und die so gestreuten Elektronen können nicht mehr kohärent mit dem elastisch-gestreuten Anteil der Elektronenwelle überlagert werden. Die inkohärent gestreuten Anteile werden innerhalb der herkömmlichen Simulation nur als Verringerung der Intensität der kohärenten Elektronenwelle durch das imaginäre Potential beschrieben.

Eine genauere Angabe des sich aufgrund von TDS ergebenden imaginären Potentials, als durch Gl. (3.13) gegeben, ist möglich, indem dieses Potential, wie auch das elastische Streupotential, als Superposition von Absorptions-Atomformfaktoren berechnet wird. Eine Parametrisierung dieser Atomformfaktoren, die auf Basis eines Einsteinmodells (die Atome vibrieren unabhängig voneinander) berechnet worden sind, ist von Weickenmeier und Kohl angegeben worden [83].

\subsubsection{Inelastische Streuung}

Unter dem Begriff inelastischer Streuung werden andere inelastische Prozesse beschrieben, die mit einem größeren Energieverlust der Elektronen verbunden sind, wie Plasmonenstreuung oder die Ionisierung der Atome. Diese Prozesse führen nur zu kleinen Streuwinkeln. Es ist daher ausreichend, sie durch einen Beitrag zum mittleren imaginären Potential zu beschreiben [83]. Ist das Mikroskop mit einem Energiefilter ausgestattet, können die inelastisch gestreuten Beiträge innerhalb der Energiebreite des Filters aus der Abbildung herausgefiltert werden.

\subsubsection{Stobbs-Faktor}

Beim Vergleich von simulierten Abbildungen, d.h. aus der Struktur simulierten Objektwellen mit angeschlossenem simuliertem Abbildungsprozess (s. Abschnitt 3.1.2), mit experimentellen Abbildungen ist eine systematische Diskrepanz zwischen beiden festgestellt worden [84]: der Kontrast (üblicherweise definiert als die Standardabweichung der Intensität) in den simulierten Abbildungen ist um den Faktor 3 bis 5 größer als in den experimentellen Abbildungen, während die Muster der Abbildungen selbst übereinstimmen. Diese Abweichung wird als Stobbs-Faktor bezeichnet. Diese Abweichungen lassen sich auch beim Vergleich von Objektwellenrekonstruktionen mit Simulationen beobachten [60].

Die Ursache dieser Abweichungen ist Gegenstand aktueller Forschung [60, 85, 86, 87, 88]. Sie treten für einen breiten Raumfrequenzbereich bei kristallinen wie auch bei amorphen Proben auf. Ihre Ursache ist nach aktuellem Stand eine Kombination vieler Prozesse: inelastische Streuprozesse und TDS innerhalb der Probe, Streuung an amorphen Oberflächenschichten von Proben und Veränderung der Proben durch Strahlenschäden. All diese Prozesse werden in den Simulationen nicht hinreichend berücksichtigt.

Objektwellen, die mittels Off-Axis-Holographie rekonstruiert werden, zeigen einen viel kleineren Stobbs-Faktor. Dies wird dem Umstand zugerechnet, dass die zur Rekonstruktion benutzten Bildanteile (Seitenbänder) nahezu ideal energiegefiltert sind (Energieverluste $<10^{-15} \mathrm{eV}$ ) 
[89]. So tragen inelastische und TDS-gestreute Elektronen hier nicht mehr zur Abbildung bei. Da ihre Beiträge im Gegensatz dazu in konventionellen Abbildungen nicht zu vernachlässigen sind, diese Beiträge aber in der Simulation nicht entsprechend berücksichtigt werden, wird ihnen ein großer Anteil am Stobbs-Faktor zugeschrieben [60].

Wie bereits in Abschnitt 3.3.2 beschrieben, werden diese inelastischen Prozesse in den üblichen Simulationsmodellen als Absorption (im Sinne von Intensitätsverringerung) beschrieben, während sie in der Wirklichkeit auch (inkohärent) zur Objektwelle beitragen. Es existieren auch Simulationsmodelle, die diese Prozesse mitbeschreiben, wie z.B. der „Frozen-Phonon“-Ansatz [90] oder „Generalized-Multislice“-Rechnungen [64]. Diese Modelle führen zwar zu einem geringerem Kontrastunterschied zwischen experimentellen und simulierten Abbildungen, beseitigen ihn allerdings auch nicht hinreichend. Der hohe Rechenaufwand dieser Methoden macht sie unpraktikabel, gerade für Simulationen mit größeren Superzellen, so dass den herkömmlichen Simulationen trotz ihrer Einschränkungen allgemein der Vorzug gegeben wird.

\subsection{Bestimmung der Linsenfehler}

\subsubsection{Symmetrische Aberrationen}

Im Diffraktogramm $|\mathcal{F}\{I(x, y)\}|$, d.h. dem Betrag der fouriertransformierten Abbildung, einer dünnen amorphen Probe lassen sich die Oszillationen des Sinus-Termes aus Gleichung (3.12) direkt beobachten, siehe Abb. 3.4. Insbesondere die Nulldurchgänge, die sog. Thon-Ringe [91], sind zu erkennen. Eine Anpassung des Polynoms $\chi_{\mathrm{s}}(\boldsymbol{q})$ ermöglicht nun die Bestimmung der Größe des Defokus, des 2-zähligen Astigmatismus und der sphärischen Aberration. Eine (semi-)automatisierte Methode für die Bestimmung der ersten beiden wurde für diese Arbeit entwickelt. Sie wird in Abschnitt 4.3.2 vorgestellt.

Diese Osziallationen sind nur in amorphen Proben zu sehen, da für kristalline Proben die Objektwelle nur für wenige $\boldsymbol{q}$ (die Braggreflexe) von 0 verschieden ist. Desweiteren streuen kristalline Proben meist stark, so dass die Vorraussetzungen des schwachen Phasenobjektes nicht mehr erfüllt sind.

Weitere Möglichkeiten zur Bestimmung dieser Aberrationen ergeben sich, wenn bereits die Rekonstruktion der Bildwelle eines schwachen Phasenobjektes vorliegt [93]. Wenn die Probe mit einer ebenen Welle beleuchtet wurde und ein reines Phasenobjekt vorliegt, sollte die Objektwelle eine konstante Amplitude haben. Die symmetrischen Aberrationskonstanten können daher durch Minimierung des Amplitudenkontrasts der um die symmetrischen Aberrationen korrigierten Objektwelle bestimmt werden. Auch kann versucht werden, die symmetrische Aberrationsfunktion selbst aus den symmetrisierten Phasen der Bildwelle im Fourierraum zu bestimmen [93, 94]. Dies erfordert allerdings Vorkenntnisse über das Objekt, so dass sich diese Methode effektiv auch wieder nur auf schwache Phasenobjekte anwenden lässt.

\subsubsection{Antisymmetrische Aberrationen}

Um die antisymmetrischen Konstanten, also die Werte für den 3-zähligen Astigmatismus und die axiale Koma zu bestimmen, sind in der Literatur verschiedene Verfahren bekannt. All diese 


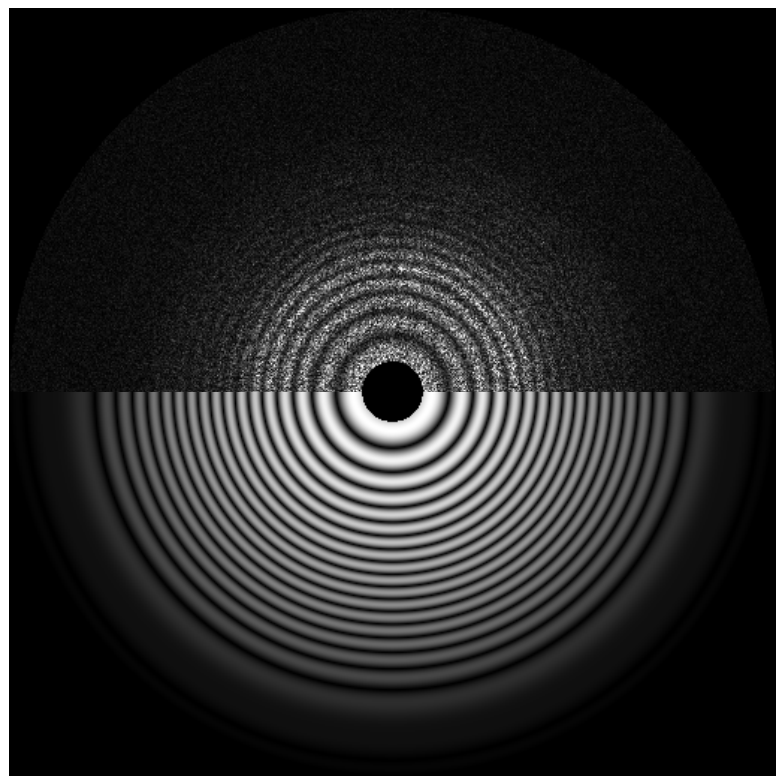

Abbildung 3.4: Obere Hälfte: Diffraktogramm von amorphem Si $\left(D=-209 \mathrm{~nm}, A_{2}=0 \mathrm{~nm}\right.$ und $C_{s}=$ $0.49 \mathrm{~mm}$ ). Die niedrigen Frequenzen sind ausgeblendet. Die Nulldurchgänge sind deutlich als Ringe zu erkennen. Untere Hälfte: Der Betrag des Imaginärteils der (symmetrisierten) CTF für diese Bedingungen (aus [92]).

Verfahren basieren darauf, dass sich die effektiven Aberrationskonstanten mit einer Strahlverkippung ändern.

Eine Verkippung des Strahls um einen Winkel $\vartheta=\lambda \boldsymbol{t}$ mit $\boldsymbol{t}=\left(t_{x}, t_{y}\right)$ gegen die optische Achse lässt sich in der Aberrationsfunktion (3.1) durch Verschiebung des Ursprungs $\boldsymbol{q} \rightarrow(\boldsymbol{q}+\boldsymbol{t})$ beschreiben. Die Aberrationsfunktion für diesen Fall lässt sich erneut in die Form der Gleichung (3.1) bringen, allerdings mit anderen Aberrationskonstanten. Diese „effektiven“ Konstanten für den verkippten Fall hängen von der Verkippung selbst und den axialen Konstanten ab.

Für den symmetrischen Anteil der Aberrationsfunktion

$$
\begin{aligned}
\chi_{\mathrm{s}}\left(q_{x}, q_{y}\right) & =\frac{1}{2} \lambda D^{\mathrm{eff}}\left(q_{x}^{2}+q_{y}^{2}\right)+\frac{1}{4} \lambda^{3} C_{s}\left(q_{x}^{2}+q_{y}^{2}\right)^{2} \\
& +\frac{1}{2} \lambda\left[A_{2 x}^{\mathrm{eff}}\left(q_{x}^{2}-q_{y}^{2}\right)+A_{2 y}^{\mathrm{eff}}\left(2 q_{x} q_{y}\right)\right]
\end{aligned}
$$

ergeben sich die effektiven Konstanten

$$
\begin{aligned}
D^{\mathrm{eff}} & =D+2 C_{s} \lambda^{2}\left(t_{x}^{2}+t_{y}^{2}\right)+4 \lambda\left[B_{x} t_{x}+B_{y} t_{y}\right] \\
A_{2 x}^{\mathrm{eff}} & =A_{2 x}+C_{s} \lambda^{2}\left(t_{x}^{2}-t_{y}^{2}\right)+2 \lambda\left[\left(A_{3 x}+B_{x}\right) t_{x}+\left(A_{3 y}-B_{y}\right) t_{y}\right] \\
A_{2 y}^{\mathrm{eff}} & =A_{2 y}+C_{s} \lambda^{2}\left(2 t_{x} t_{y}\right)+2 \lambda\left[\left(A_{3 y}+B_{y}\right) t_{x}-\left(A_{3 x}-B_{x}\right) t_{y}\right] .
\end{aligned}
$$

Im asymmetrischen Anteil hängen die effektiven Konstanten für die Bildverschiebung $\left(A_{1 x}^{\text {eff }}\right.$, $\left.A_{1 y}^{\text {eff }}\right)$ von sämtlichen Linsenfehlern ab, während sich die effektiven Konstanten für die Koma nur mit der sphärischen Aberration ändern. Für die genauere Angabe dieses Anteiles sei auf die Literatur verwiesen (z.B. [67])

Ein Verfahren zur Messung der Linsenfehler (siehe z.B. [95]) nutzt aus, dass sich die Abbildungen mit Strahlverkippung verschieben. Durch eine Kreuzkorrelation der verkippten mit 
der axialen Abbildung wird diese Verschiebung $\left(A_{1 x}^{\mathrm{eff}}, A_{1 y}^{\mathrm{eff}}\right)$ gemessen. Aus den Bildverschiebungen der verschiedenen Kippwinkel lassen sich anschließend die axialen Konstanten berechnen. Für diese Methode werden vorwiegend amorphe Proben benötigt, da sich von kristallinen Proben aufgrund der Translationssymmetrie nur Bildverschiebungen modulo der Gitterkonstanten messen lassen. Ein Nachteil des Verfahrens ist die Verfälschung der Messung durch Probendrift.

In anderen Verfahren, wie auch in dem in dieser Arbeit verwendeten (s. Abschnitt 4.3), werden Diffraktogramme von amorphen Proben für verschiedene Strahlverkippungen aufgenommen [96]. Eine solche Aufstellung von Diffraktogrammen wird als Zemlin-Tableau bezeichnet. Wie im letzten Abschnitt beschrieben, lassen sich aus den einzelnen Diffraktogrammen die effektiven Konstanten für den Defokus und den 2-zähligen Astigmatismus bestimmen. Aus diesen Werten für verschiedene Verkippungen können unter Umkehrung der Gleichungen (3.15) die axialen Konstanten bestimmt werden. Anders als die oben vorgestellte Methode ist diese unempfindlich gegen Probendrift.

Ein aufwendigeres Verfahren wurde von Meyer vorgeschlagen [97]: Für dünne amorphe Proben lässt sich die Bildwelle durch filterbasierte Rekonstruktionsmethoden aus wenigen Abbildungen (z.B. drei) rekonstruieren. Die Messung der symmetrischen effektiven Konstanten ist nun direkt aus der Wellenfunktion möglich. Werden die Bildwellen für verschiedene Strahlverkippungen rekonstruiert, lassen sich aus den so erhaltenen effektiven Konstanten wiederum die axialen Konstanten bestimmen.

Ausführliche Übersichten über die verschiedenen Verfahren sind in den Artikel von Typke et al. [67] und Kirkland et al. [98] zu finden. 


\section{Objektwellenrekonstruktion}

Bei der Objektwellenrekonstruktion wird die komplexe Objektwellenfunktion, d.h. die Elektronenwelle auf der Rückseite der Probe, rekonstruiert. Wie in Abschnitt 3.1 beschrieben wurde, stehen die HRTEM-Abbildungen selbst in einem komplexen Zusammenhang zu dieser Welle. Werden statt den Abbildungen Objektwellen analysiert, so muss dieser komplexe Zusammenhang nicht in der Interpretation der Messungen berücksichtigt werden. Desweiteren ist die Abbildung eine Intensitätsmessung, die nicht sensitiv auf die Phase der komplexen Bildwelle ist. Diese Information wird bei der Objektwellenrekonstruktion zurückgewonnen.

Der Unterschied zwischen HRTEM-Abbildungen und der Objektwelle wird in Abb. $4.1 \mathrm{am}$ Beispiel von GaN (4.8 nm Dicke) demonstriert (Simulation mit den Parametern des verwendeten Elektronenmikroskops). Die Lage der Intensitätsmaxima hängt von der Probendicke und dem Defokus ab, sie können nicht nur auf einer der beiden Atomsorten liegen, sondern auch in den „Löchern“ der Struktur auftreten, wie z.B. bei $D=-30 \mathrm{~nm}$. Bei $D=-45 \mathrm{~nm}$ liegen die Intensitätsmaxima zwar fast auf den Orten der Ga-Atome, allerdings ist die Abweichung in jeder der beiden (0002)-Halbebenen unterschiedlich (das Maximum ist in der einen Ebene weiter links, in der anderen weiter rechts als das Ga-Atom). In der Objektwelle hingegen sind die Lagen der Ga-Atome immer gut auszumachen, da sie um ein Vielfaches stärker streuen als die N-Atome. Folglich lässt sich auch die Stapelfolge immer klar erkennen.
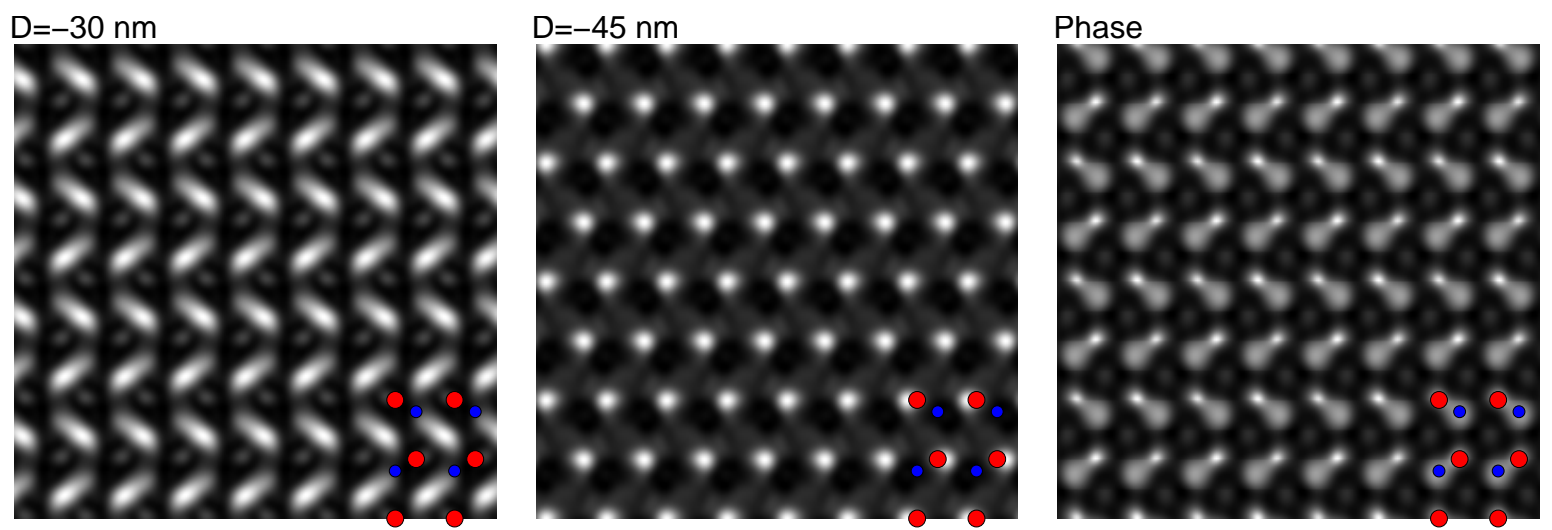

Abbildung 4.1: Simulation einer $4.8 \mathrm{~nm}$ dicken GaN-Schicht. Die roten Kreise geben Positionen der Ga-Atome, die blauen die der N-Atome an. Die beiden linken Abbildungen sind zu erwartende HRTEMAbbildungen bei verschiedenen Fokuswerten. Die rechte Abbildung ist die Phase der Objektwelle, wie sie bei der Objektwellenrekonstruktion zu erwarten wäre.

In diesem Kapitel werden die einzelnen Schritte beschrieben, die bei der Objektwellenrekonstruktion notwendig sind:

1. Die Aufnahme einer Defokusserie (10-20 Abbildungen bei unterschiedlichem Defokus), aus der die Objektwelle rekonstruiert wird, sowie die Aufnahme eines Zemlin-Tableaus, 
aus dem die Linsenfehler bestimmt werden können. Ein Zemlin-Tableau besteht aus mehreren Aufnahmen eines amorphen Probenbereichs bei verschiedenen Kippwinkeln des Elektronenstrahls. Details für diese Aufnahmen sind im Abschnitt 4.1 beschrieben.

2. Aufgrund von thermischen Instabilitäten lässt es sich nicht vermeiden, dass die Probe während der Aufnahme der Defokusserie driftet. Die Drift, die während der eigentlichen Belichtung der Einzelabbildungen auftritt, ist jedoch vernachlässigbar. Über die gesamte Serie kommt es allerdings zu Bildverschiebungen. Die Verschiebungen müssen gemessen und berücksichtigt werden, die Vorgehensweise dafür beschreibt Abschnitt 4.2.

3. Die Rekonstruktion der Objektwelle wird von einem Softwarepaket übernommen ("TrueImage Fokusserienrekonstruktions Software" der Firma FEI). Diese Software basiert auf der SD-MAL Implementation von Coene et al. und nutzt die Paraboloidmethode zum Berechnen der Startwerte (s. auch Abschnitt 3.2) [72, 99]. In dieser Software wird bereits die Bildwelle um die sphärische Aberration, den Defokus und die Effekte der partiellen Kohärenz korrigiert. Die Effekte der anderen Linsenfehler, die sog. residualen Aberrationen, sind weiterhin in der Welle enthalten. Dabei sind ihre Effekte klein genug, um nicht bei der Korrektur der Effekte der eingeschränkten räumlichen Kohärenz berücksichtigt werden zu müssen [68].

4. Das Vorgehen zum Ermitteln der Größe der Linsenfehler aus den Zemlin-Tableaus wird in Abschnitt 4.3 beschrieben. Dazu werden in den einzelnen Diffraktogrammen die effektiven Werte für Defokus und 2-zähligen Astigmatismus bestimmt. Aus diesen Werten und der Strahlverkippung des jeweiligen Diffraktogramms, lassen sich dann die axialen Aberrationskonstanten bestimmen.

5. Die residualen Aberrationen, deren Größe im letzten Schritt ermittelt wurden, werden abschließend numerisch in der Objektwelle korrigiert.

Das Kapitel schließt mit einer Messung der Objektwelle eines Si-Kristalls ab. Diese Messung soll als Test dafür dienen, ob die Objektwellenrekonstruktion erfolgreich durchgeführt werden kann.

\subsection{Experimentelles Vorgehen}

Die Defokusserien wurden mit einer Belichtungszeit von 0.5 Sekunden pro Bild aufgenommen und bestanden typischerweise aus 20 Abbildungen. Mit der benutzten CCD-Hardware ließen sich so Aufnahmen im Abstand von neun Sekunden durchführen, d.h. die Aufnahme einer kompletten Defokusserie dauert etwa drei Minuten. Eine Vorraussetzung der verwendeten Implementierung für die Rekonstruktion ist, dass die Aufnahmen in der Serie eine konstante Defokusschrittweite haben müssen. Diese Schrittweite selbst sollte dabei von der Größe der Streubreite $\Delta$ des Defokus sein (beim verwendeten Mikroskop $\Delta=4.0 \mathrm{~nm}$ ). Die Serie wurde mit einer Schrittweite von $-3.4 \mathrm{~nm}$ aufgenommen, d.h. von kleineren zu größeren Unterfokuswerten hin. Dies ist die mögliche Schrittweitenwahl des Mikroskops, die $\Delta$ am nächsten liegt. Die Größe dieser Schrittweite des Mikroskops wurde in unabhängigen Messungen bestimmt, indem für verschiedene Defokusserien von amorphen Proben die Defokuswerte aus den Diffraktogrammen der Einzelabbildungen bestimmt wurden. 
Die absoluten Werte des Defokus wurden so gewählt, dass die Serie bei Werten von $D=$ $-80 \mathrm{~nm}$ bis $D=-120 \mathrm{~nm}$ anfangen. Damit liegt die Serie um den „optimalen“ Defokus ${ }^{1} D_{\text {opt }}$, bei dem eine minimale optische Delokalisation aufgrund der Linsenfehler auftritt [100]. Für das verwendete Mikroskop $D_{\mathrm{opt}}=-148 \mathrm{~nm}$. Näheres zur Wahl des absoluten Defokus für die Defokusserienrekonstruktion ist bei Thust et al. zu finden [99].

Eine Bestimmung des absoluten Defokus am Mikroskop selbst ist nicht mit hinreichender Genauigkeit möglich. Die Abweichung vom wahren Defokus lässt sich wie die residualen Aberrationen behandeln. Der wahre Defokus lässt sich bei kristallinen Proben meist nur durch Vergleiche der rekonstruierten Objektwelle mit Simulationen ermitteln.

Für die Messung der Linsenfehler wurde zusätzlich zu jeder Defokusserie auch ein ZemlinTableau aufgenommen. Dazu wurde die Probe nach Aufnahme der Defokusserie an eine amorphe Stelle (z.B. Klebstoff oder Probenrand) verfahren. Die Bildverschiebung wurde dabei nicht verändert, da sie einen Einfluss auf die auftretenden Bildfehler hat. Die Tableaus wurden bei einer Kippung des Strahls gegen die optische Achse von $\sim 0.3^{\circ}$ aufgenommen. Für ein Tableau wurden acht Aufnahmen mit $45^{\circ}$ Azimuthwinkelschrittweite gemacht, was eine Überbestimmung der Aberrationkonstanten erlauben soll. Die Beleuchtung wurde für jede Aufnahme des Tableaus jeweils zentriert, um eine möglichst parallele Beleuchtung zu erreichen. Die Serie selbst wurde mit einer zentrierten Beleuchtung entlang der optischen Achse aufgenommen. Die Tableaus wurden aus Gründen, die später erläutert werden, bei starkem Unterfokus $(D=-150$ bis $-200 \mathrm{~nm})$ aufgenommen.

\subsection{Messung der Bildverschiebung}

Aufgrund von Drift kommt es zu einer Bildverschiebung über die Aufnahmen der Serie. Diese Verschiebung lässt sich bei der Defokusserienrekonstruktion einfach berücksichtigen, indem in jeder Aufnahme nur der entsprechende Bildausschnitt verwendet wird. Dazu muss allerdings die Verschiebung zunächst gemessen werden.

Bei kristallinen Proben wird die Messung der Verschiebung durch die Translationssymmetrie des Gitters erschwert, bei der definitionsgemäß nur Verschiebungen bis auf Vielfache der Gitterkonstante existieren. Während das Gitter anteilsmäßig am stärksten zur Abbildung beiträgt, sind es daher gerade die schwächeren nichtperiodischen Beiträge, wie Kristalldefekte und amorphe Deckschichten, die bei der Messung zu detektieren sind.

Außerdem ist bei der Messung zu beachten, dass sich die Abbildungen selbst wegen des veränderten Defokus über die Serie ändern. Dies erschwert insbesondere in nicht-zentrosymmetrischen Projektionen, z.B. GaN [11200], die Messung: Bei zentrosymmetrischen Projektionen ist die Verschiebung des Gitters in der Abbildung gegenüber dem Gitter in der Objektwelle unabhängig vom Defokus, bei nicht-zentrosymmetrischen Proben hingegen hängt sie vom Defokus ab.

Es reicht aus, wenn die Verschiebungen auf wenige Bildpunkte (Pixel) genau gemessen werden, da während der Rekonstruktion die Software weitere kleine Korrekturen der Verschiebung auf Subpixel-Niveau vornimmt. Die hohe Genauigkeit dieser Korrekturen wird durch einen

\footnotetext{
${ }^{1}$ Dieser Fokus wird teilweise auch Lichte-Fokus genannt. Er ist nicht zu verwechseln mit dem Scherzerfokus, der von manchen Autoren ebenfalls als optimaler Fokus bezeichnet wird.
} 
Vergleich der Aufnahmen mit einer aus der rekonstruierten Welle berechneten Abbildung erreicht. In diesem Vergleich wird die Änderung des Defokus explizit berücksichtigt.

Üblicherweise wird die Kreuzkorrelationfunktion

$$
X(\boldsymbol{r})=\mathcal{F}^{-1}\left\{\widetilde{I}(\boldsymbol{q}) \cdot \widetilde{J}^{*}(\boldsymbol{q})\right\}
$$

benutzt, um Verschiebungen zu messen [101]. $\widetilde{I}(\boldsymbol{q})$ und $\widetilde{J}(\boldsymbol{q})$ sind zwei fouriertransformierte Abbildungen. Die Kreuzkorrelation von zwei nicht-periodischen Abbildungen hat ein scharfes Maximum an dem Ort, der dem inversen Verschiebungsvektor zwischen den Abbildungen entspricht. Leider ist die Kreuzkorrelationfunktion sehr sensitiv auf die periodischen Anteile in den Abbildungen, wie in Abb. 4.2c zu erkennen ist. Diese Anteile tragen zwar nur bei wenigen Raumfrequenzen zur Kreuzkorrelation bei, dafür sind diese Beiträge aber sehr stark gegenüber den nichtperiodischen Anteilen, die bei allen Raumfrequenzen zu den Abbildungen beitragen.

Meyer schlug daher eine andere Korrelationsfunktion für die Ausrichtung der Bilder einer Serie vor [76], die auch in dieser Arbeit verwendet wird: die Phasenkorrelationsfunktion

$$
P(\boldsymbol{r})=\mathcal{F}^{-1}\left\{\frac{\widetilde{I}(\boldsymbol{q}) \cdot \widetilde{J}^{*}(\boldsymbol{q})}{\left\|\widetilde{I}(\boldsymbol{q}) \cdot \widetilde{J}^{*}(\boldsymbol{q})\right\|+\eta} f(\boldsymbol{q})\right\} .
$$

Dabei ist $\eta$ eine kleine Zahl, die verhindern soll, dass der Nenner verschwindet. Die Stufenfunktion $f(q)$ ist für Raumfrequenzen 0, die größer als das Informationslimit qinfo des Mikroskops sind, und für kleinere Frequenzen 1. Die hohen Frequenzen tragen keine Bildinformationen, sondern nur Rauschen.

Die Phasenkorrelationsfunktion bewertet alle Raumfrequenzen $\left(q<q_{\text {info }}\right)$ in gleichem Maße, indem die Intensität für alle gleich gesetzt wird. Daher ist sie kaum sensitiv auf periodische Anteile, die nur bei wenigen Raumfrequenzen Beiträge liefern. Diese geringe Sensitivität ist im Vergleich zur Kreuzkorrelation in Abb. 4.2 gut zu sehen.

In Abb. 4.3 ist der Verlauf der Bildverschiebungen über zwei Defokusserien zu sehen. Die gesamte Drift über die Serie beträgt etwa 2 bis $2.5 \mathrm{~nm}$. Umgerechnet auf die Drift pro Belichtung (0.5 zu 171 Sekunden), ergibt sich eine mittlere Drift von unterhalb $0.1 \AA$ während der Einzelaufnahme. Damit liegt sie weit unter dem Informationslimit und kann daher vernachlässigt werden.

\subsection{Messung der Linsenfehler}

Für die Korrektur der residualen Aberrationen ist es notwendig, ihre Größe zu bestimmen. Eine Abschätzung der benötigten Genauigkeit dieser Bestimmung wird in Abschnitt 4.3.1 gegeben. Die axialen Aberrationskonstanten für den 2-zähligen Astigmatismus, $A_{2 x}$ und $A_{2 y}$, für den 3-zähligen Astigmatismus, $A_{3 x}$ und $A_{3 y}$, sowie für die Koma, $B_{x}$ und $B_{y}$, sollen bestimmt werden. Der Wert für die sphärische Aberration ist eine Konstante des Mikroskops und ändert sich nicht, er muss daher nicht bei veränderten Abbildungsbedingungen neu bestimmt werden. 
(a)

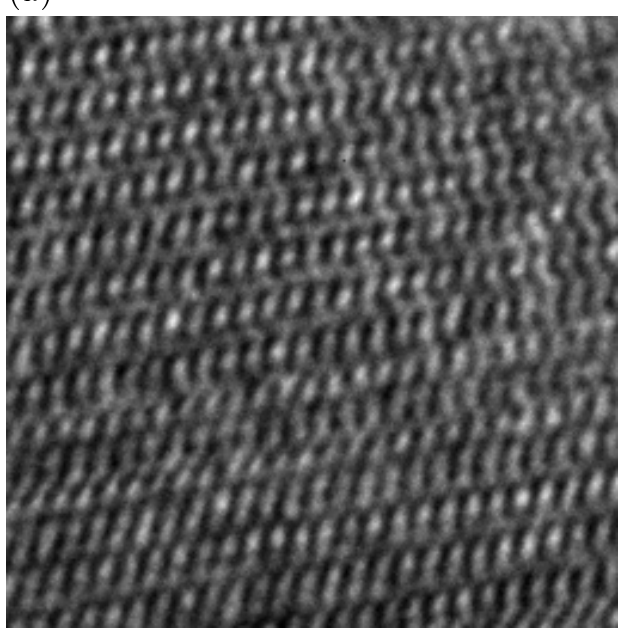

(c)

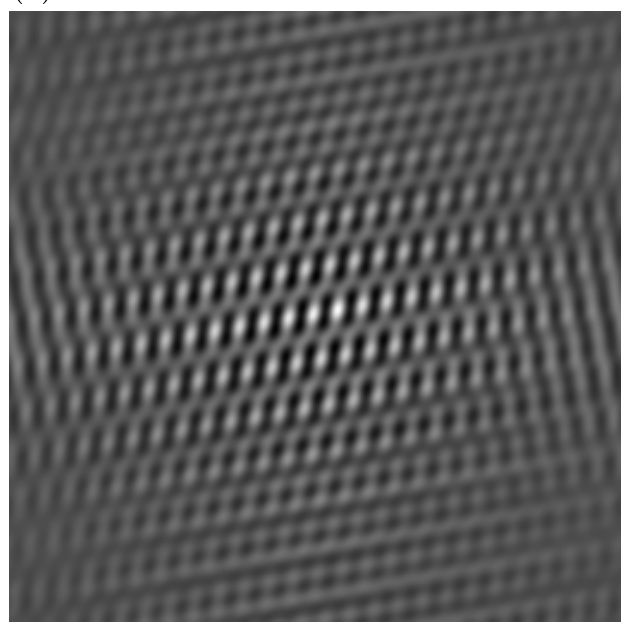

(b)

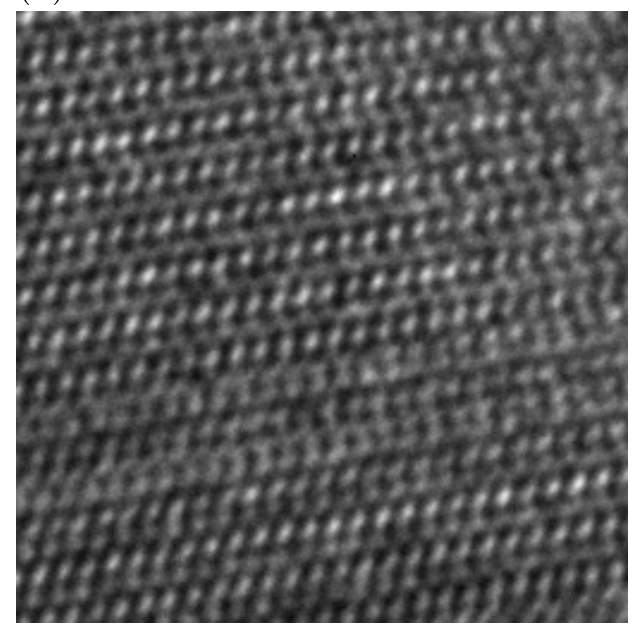

(d)

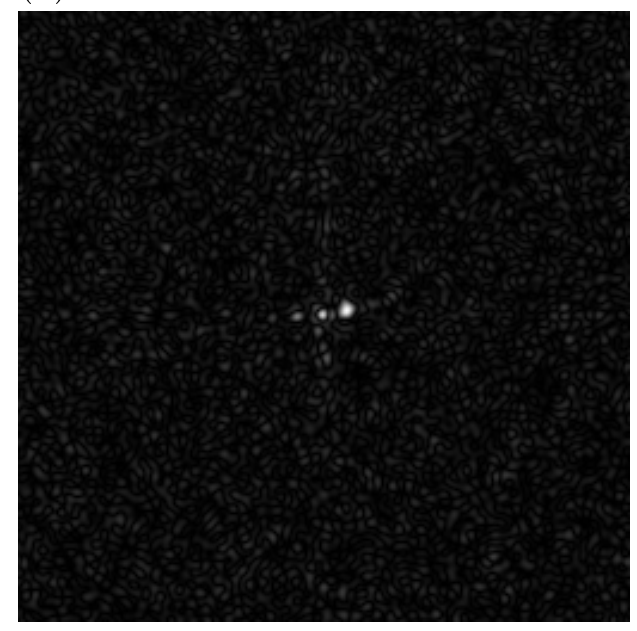

Abbildung 4.2: Vergleich von Phasenkorrelation und Kreuzkorrelation bei der Messung der Bildverschiebung zwischen zwei Aufnahmen aus einer Defokusserie von GaN [112̄0]: (a) Aufnahme bei $D=-111.6 \mathrm{~nm}$. (b) Aufnahme bei $D=-115.0 \mathrm{~nm}$. (c) Kreuzkorrelation beider Aufnahmen. Aufgrund der starken Periodizität des Gitters lässt sich das Maximum schwer erkennen. (d) Phasenkorrelationsfunktion beider Aufnahmen. Das Maximum ist deutlich zu erkennen. 


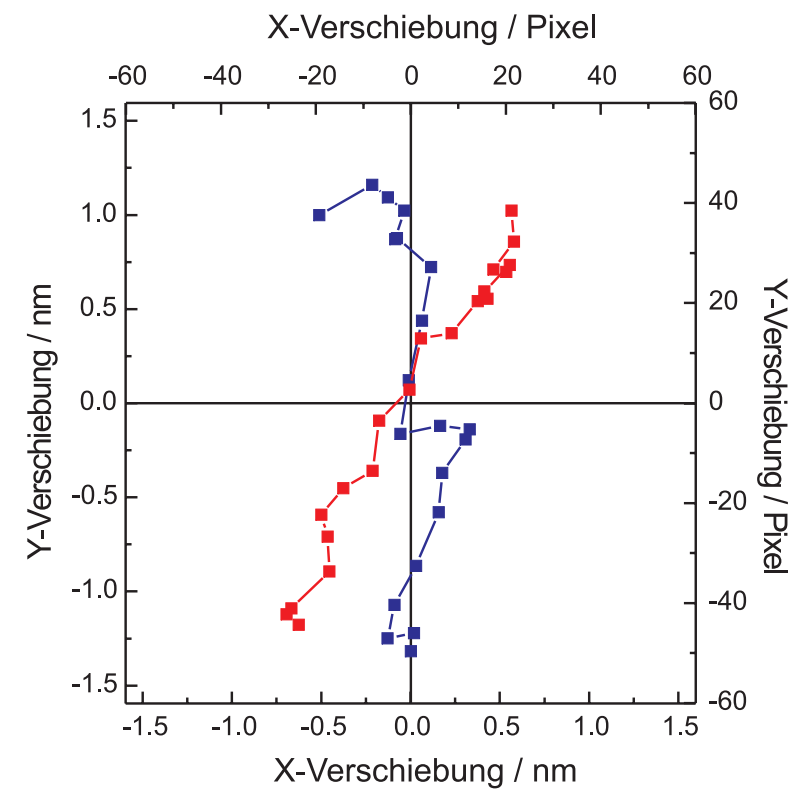

Abbildung 4.3: Bildverschiebungen in zwei typischen Defokusserien relativ zu einem willkürlichen Ursprung.

Das Vorgehen zur Bestimmung der Konstanten ist es, zunächst aus mehreren, bei verschiedenen Kippwinkeln aufgenommenen Diffraktogrammen einer amorphen Probenstelle die jeweiligen effektiven Konstanten für den Defokus und den 2-zähligen Astigmatismus zu bestimmen. Das dazu benutzte Verfahren wird in Abschnitt 4.3.2 vorgestellt.

Diese Bestimmung wird für eine größere Anzahl an Diffraktogrammen (meistens acht) vorgenommen, um eine hinreichende Überbestimmung der axialen Konstanten zu erlauben. Wie in Abschnitt 4.3.3 beschrieben, werden anschließend mit einer $\chi^{2}$-Minimierung aus den verschiedenen Effektivwerten bei Strahlverkippung die axialen Konstanten bestimmt.

Mit diesem Vorgehen ist es zwar auch möglich den absoluten Defokus für das Zemlin-Tableau zu bestimmen, allerdings wurde die zu korrigierende Defokusserie an einer anderen Probenstelle aufgenommen. Da sich der absolute Defokus mit der Probendicke und -höhe ändert, ist im Allgemeinen nicht davon auszugehen, dass er an dem Ort, an dem das Zemlin-Tableau aufgenommen wurde, der gleiche ist wie am Ort der Serie. Der residuale Defokus, also die Abweichung des nominellen Defokus, bei dem die Serie rekonstruiert wurde, vm tatsächlichen Defokus, lässt sich daher besser über den Vergleich mit Bildsimulationen oder auf anderen Wegen bestimmen.

\subsubsection{Abschätzung der benötigten Genauigkeit}

Die benötigte Genauigkeit, mit der die Linsenfehler gemessen werden müssen, hängt davon ab, wie groß der verbleibende Fehler sein darf. Als Fehler wird dabei die maximal erlaubte Phasenverschiebung innerhalb einer gegebenen Apertur verwendet. Es soll dabei maximal eine Phasenschiebung von $\pi / 4$ erlaubt werden, was sich bei Vergleichen von Bildsimulationen als ein recht konservatives Kriterium herausgestellt hat [102]. 
Dieses Kriterium führt für die Aberrationskonstanten zu der Bedingung

$$
2 \pi \chi\left(q_{\text {info }}\right)<\frac{\pi}{4}
$$

Diese Bedingung ergibt für die einzelnen residualen Aberrationen, dass sie kleiner sein müssen als die folgenden Werte (für das verwendete Mikroskop gilt $q_{\text {info }}=8 \mathrm{~nm}^{-1}$ ):

\begin{tabular}{lccc} 
Aberrationskonstanten & $q_{\text {info }}=7 \mathrm{~nm}^{-1}$ & $q_{\text {info }}=8 \mathrm{~nm}^{-1}$ & $q_{\text {info }}=10 \mathrm{~nm}^{-1}$ \\
\hline \hline Defokus $D$ & $2.0 \mathrm{~nm}$ & $1.6 \mathrm{~nm}$ & $1.0 \mathrm{~nm}$ \\
2-zähliger Astigmatismus $A_{2}$ & $2.0 \mathrm{~nm}$ & $1.6 \mathrm{~nm}$ & $1.0 \mathrm{~nm}$ \\
3-zähliger Astigmatismus $A_{3}$ & $174 \mathrm{~nm}$ & $116 \mathrm{~nm}$ & $60 \mathrm{~nm}$ \\
Axiale Koma $B$ & $58 \mathrm{~nm}$ & $39 \mathrm{~nm}$ & $20 \mathrm{~nm}$ \\
Sphärische Aberration $C_{s}$ & $13.2 \mu \mathrm{m}$ & $7.7 \mu \mathrm{m}$ & $3.2 \mu \mathrm{m}$ \\
\hline
\end{tabular}

Dabei wurden allerdings die kumulativen Effekte der Linsenfehler außer acht gelassen, was die konservative Wahl der Phasenschiebung von $\pi / 4$ rechtfertigt. Die Werte in der obigen Tabelle können als benötigte Genauigkeit interpretiert werden, da die untersuchte Welle bis auf die Genauigkeit innerhalb der bestimmten Konstanten korrigiert wird.

\subsubsection{Bestimmung von Defokus und Astigmatismus}

Wie in Abschnitt 3.4.1 bereits beschrieben wurde, ist es möglich, den Defokus und den 2zähligen Astigmatismus in Diffraktogrammen von amorphen Proben zu bestimmen. Dazu wird eine berechnete Kontrasttransferfunktion, Gl. (3.6), an die Diffraktogramme angepasst, indem die Größen $D, A_{2 x}$ und $A_{2 y}$ verändert werden.

Im folgenden Abschnitt beziehen sich die Begriffe Defokus und Astigmatismus immer auf die im Diffraktogramm sichtbaren effektiven Werte für Defokus und 2-zähligen Astigmatismus.

In diesem Abschnitt wird eine Methode für diese Anpassung an die Diffraktogramme vorgestellt. Dabei arbeitet diese Methode nicht vollautomatisch, sondern bedarf weiterhin einer groben Anpassung der Startwerte per Hand.

Bei der Anpassung der Diffraktogramme amorpher Proben, können verschiedene Schwierigkeiten auftreten:

- Die verwendeten amorphen Materialien bestehen meist aus leichten Elementen (z.B. der Klebstoff vorwiegend aus Kohlenstoff). Ihr Streusignal ist recht schwach für größere Winkel, also höhere Raumfrequenzen. Außerdem führt die Strahlverkippung zu einer geringeren Kohärenz des Elektronenstrahls, was ebenfalls zu einer Dämpfung der hohen Frequenzen führt. Eine Anpassung muss daher im Bereich tiefer Frequenzen $\left(q<q_{\max } \approx\right.$ $4-5 \mathrm{~nm}^{-1}$ ) vorgenommen werden.

- Für zu tiefe Raumfrequenzen ist die CTF nicht empfindlich gegenüber Änderungen des Defokus und des Astigmatismus, außerdem wird dieser Frequenzbereich vom Nullstrahl überlagert. Daher wird auch dieser Frequenzbereich bei der Anpassung ignoriert $(q>$ $q_{\min } \approx 1-1.5 \mathrm{~nm}^{-1}$ ). 
(a)

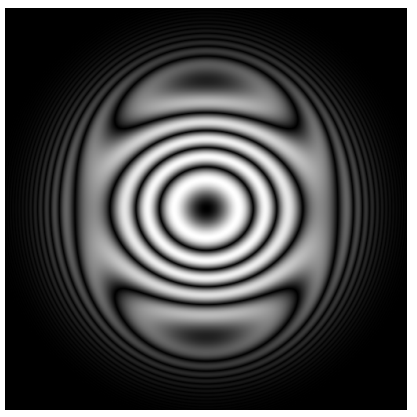

(b)

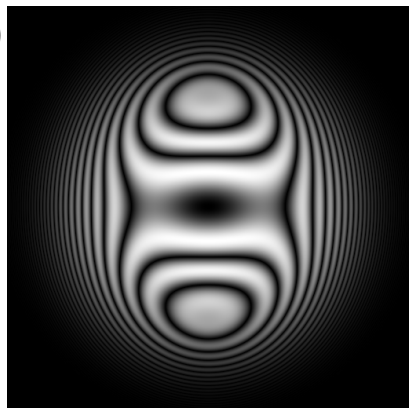

(c)

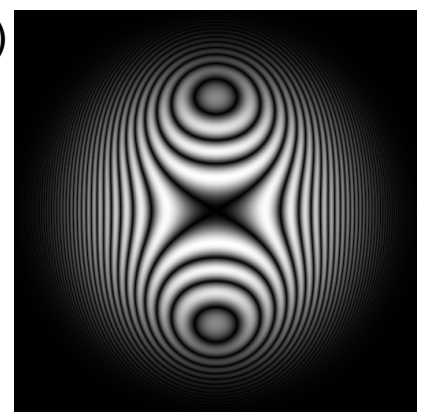

Abbildung 4.4: Verschiedene Formen von Thonringen. Dargestellt ist der Betrag der entsprechenden CTF. (a) Elliptische Form; (b) Zwischenform; (c) Kreuz-Form oder hyperbolische Form.

- Die Nulldurchgänge der CTF können eine elliptische Form, eine kreuzförmige (hyperbolische) Form oder eine Zwischenform (parabolische Form) aufweisen (s. Abb. 4.4).

- Bei Defokuswerten im Bereich des Gaußschen Fokus oder des Scherzerfokus sind meist keine Thon-Ringe für den angepassten Raumfrequenzbereich zu erkennen. Bei kleinen Defokuswerten kann es zudem schwierig sein, zwischen Unter- $(D<0)$ und Überfokus $(D>0)$ sowie zwischen zwei zueinander senkrechten Azimuthalwinkeln des Astigmatismus zu unterscheiden. Um diese Schwierigkeit zu vermeiden, sollten die Tableaus möglichst bei großem Unterfokus ( $D=-150 \mathrm{~nm}$ bis $-200 \mathrm{~nm}$ ) aufgenommen werden.

- In der Abbildung könnten sich kristalline Bereiche befinden. Diese führen zu starken Reflexen im Diffraktogramm, die das Ringmuster überlagern.

Ein einfaches Verfahren zur Bestimmung des Defokus kann in astigmatismusfreien Diffraktogrammen angewendet werden. Hier sind die Nulldurchgänge der CTF kreisförmige Ringe. Aus einer azimuthalen Mittelung oder auch durch einen Schnitt durch das Diffraktogramm lässt sich eine eindimensionale Darstellung der Intensitäten $\widetilde{I}(q)$ gewinnen. Die leicht auffindbaren Minima in dieser Darstellung entsprechen den Nullstellen der berechneten CTF. Dies ist ein Standardverfahren zur Bestimmung des Defokus (und auch der sphärischen Aberration) und in vielen Elektronenmikroskopiebüchern zu finden (z.B. [69]).

Ein Ansatz zur Bestimmung von Defokus und Astigmatismus ist die Erweiterung dieses eindimensionalen Verfahrens: Es kann gezeigt werden, dass der kumulative Effekt von Astigmatismus und Defokus einem unterschiedlichen Defokus in zwei zueinander senkrechten Richtungen entspricht. In der einen Richtung findet sich dabei der Defokus $D+A_{2}$ und in der dazu senkrechten $D-A_{2}$. Der Azimuthalwinkel des Astigmatismus gibt dabei die Richtung dieser Achsen an. Wird nun der Azimuthalwinkel, z.B. durch den Vergleich des Diffraktogramm mit seinem Spiegelbild, bestimmt [103], lassen sich die Defokuswerte für diese beiden Hauptrichtungen bestimmen und daraus wieder $D, A_{2 x}$ und $A_{2 y}$ berechnen.

Für Diffraktogramme mit elliptischer Form der Thonringe ergeben sich zwei weitere Möglichkeiten: Die eine basiert auf einer azimuthalen Mittelung innerhalb von Sektoren des Diffraktogramms (von z.B. $7.5^{\circ}$ ). In jedem Sektor lässt sich dann wieder der Defokus bestimmen [104]. Der Defokus eines Sektors hängt in diesem Fall über den Azimuthalwinkel des Sektors von 
den Konstanten $D, A_{2 x}$ und $A_{2 y}$ ab. Eine andere Möglichkeit besteht darin, zunächst über Bilderkennung den Azimuth und die Exzentrität der Ellipsen zu bestimmen und anschließend eine elliptischen Mittelung des Diffraktogrammes durchzuführen [105]. Aus der Mittelung lässt sich dann der Defokus bestimmen und zusammen mit der Ellipsenform die Größe des Astigmatismus berechnen.

In dem hier vorgestellten Verfahren soll ein anderer Ansatz benutzt werden, um den Defokus und den Astigmatismus in möglichst vielen Fällen zu bestimmen: Eine berechnete zweidimensionale CTF soll an das Diffraktogramm angepasst werden, wie bereits von Uhlemann und Haider vorgeschlagen wurde [102].

\subsubsection{Vorbetrachtung}

Ein simpler Vergleich zwischen gemessenem Diffraktogramm und berechneter CTF ist nicht möglich, da verschiedene Einflüsse berücksichtigt werden müssen, die in diesem Unterabschnitt betrachtet werden.

Für die Näherung des schwachen Phasenobjekts nimmt das Diffraktogramm die Form der Gleichung (3.12) an. Ausgehend von dieser Gleichung lässt sich das Diffraktrogramm modellieren als

$$
\|\widetilde{I}(\boldsymbol{q})\| \approx \mathcal{E}(q)\left|\sin \left(-2 \pi \chi_{\mathrm{s}}(\boldsymbol{q})\right)\right|+\mathcal{B}(q) \text { für } q>q_{\min } .
$$

Der Term $\mathcal{B}(q)$ beschreibt dabei den Hintergrund des Diffraktogramms, hier gehen das Rauschen und diffus gestreute Anteile des Elektronenstrahls ein. Auch Abweichungen von der Näherung des schwachen Phasenobjektes tragen zum Hintergrund bei. Die Einhüllende $\mathcal{E}(q)$ beschreibt zum einen den Einfluss der Probe selbst, d.h. des Strukturfaktors und der Atomformfaktoren des amorphen Materials, zum anderen die Einflüsse der eingeschränkten Kohärenz. Beide Funktionen lassen sich als rotationssymmetrisch annehmen.

Der Vergleich zwischen den experimentellen Diffraktogrammen und der berechneten Kontrasttransferfunktion bedarf einer Abschätzung für $\mathcal{E}(q)$ und $\mathcal{B}(q)$. Da die Dämpfungsfunktionen der CTF bereits in die Einhüllenden $\mathcal{E}(q)$ einfließen, beschränkt sich die Anpassung auf die Anpassung der zweidimensionalen Aberrationsfunktion $\chi_{\mathrm{s}}(\boldsymbol{q})$, Gl. (3.14).

\subsubsection{Vorverarbeitung der Diffraktogramme}

Die Diffraktogramme der aufgenommenen Abbildungen werden mittels des FFT-Algorithmus berechnet. Dieser Algorithmus berechnet strenggenommen eine Fourierreihe, geht also von einer periodischen Fortsetzung der Abbildung an seinen Rändern aus. Die Abbildung ist jedoch nur ein Ausschnitt und somit nicht periodisch. Dies führt zu Unstetigkeiten bei der periodischen Fortsetzung am Rand, die sich in Artefakten in den Diffraktogrammen (Streaking) äußern.

Eine Methode diese Artefakte zu vermindern, wäre die Anwendung von Fensterfunktionen, die nachteiligerweise auch einen Effekt auf das Diffraktogramm selbst haben. Für die Anpassung der Aberrationsfunktion wird stattdessen vor der Anwendung der FFT eine Intensitätsebene an die Intensitätswerte der Abbildung angepasst und von der Abbildung abgezogen. Die Änderungen des Diffraktogramms aufgrund dieses Vorgehens beschränken sich auf die tiefen Raumfrequenzen $q<q_{\min }$. 


\subsubsection{Abschätzung des Hintergrundes $B(q)$ und der Einhüllenden $\mathcal{E}(q)$}

Zur Abschätzung des Hintergrundes wird die empirische Formel

$$
B(q)=\exp \left\{-b_{1}-b_{2} \sqrt{q}-b_{3} q-b_{4} q^{2}\right\},
$$

verwendet, die von Mallick [105] für die automatisierte CTF Anpassung vorgeschlagen wurde. Dabei werden die $b_{i}$ durch Minimierung von

$$
S=\int_{q_{\min } \leq q \leq q_{\max }} \frac{\mathrm{d} \boldsymbol{q}^{2}}{q}\left[\ln \|\widetilde{I}(\boldsymbol{q})\|-b_{1}-b_{2} \sqrt{q}-b_{3} q-b_{4} q^{2}\right]^{2}
$$

unter der Nebenbedingung $\|\widetilde{I}(\boldsymbol{q})\| \geq \mathcal{B}(q)$ für alle $q_{\text {min }} \leq q \leq q_{\text {max }}$, quasi von "unten" (s. Abb. 4.5), an die experimentellen Diffraktogramme angepasst. Zur Anpassung wurde der in der IDL-Software implementierte GRG2-Algorithmus verwendet [106].

Für die Abschätzung der Einhüllenden wird die gleiche empirische Formel verwendet:

$$
\mathcal{E}(q)=\exp \left\{-m_{1}-m_{2} \sqrt{q}-m_{3} q-m_{4} q^{2}\right\} .
$$

Zur Anpassung schlägt Mallick vor, diese Formel ähnlich wie im letzten Abschnitt an $\|\widetilde{I}(\boldsymbol{q})\|-\mathcal{B}(q)$ anzupassen, diesmal allerdings unter der Nebenbedingung $\mathcal{E}(q) \geq\|\widetilde{I}(\boldsymbol{q})\|-\mathcal{B}(q)$. Diese Anpassung von „oben“ an die Diffraktogramme (s. Abb. 4.5) ist sicherlich sehr gut geeignet, solange sich keine Kristallite in der Aufnahme befinden. Kristalline Bereiche führen zu Reflexen im Diffraktogramm, die typischerweise große Betragswerte auf einem kleinen Frequenzbereich haben, was dazu führt, dass die Anpassung mit Nebenbedingung auf den Maxima der Reflexe „hängenbleibt“.

Da der Frequenzbereich, auf dem sich die Reflexe finden, klein gegenüber dem gesamten zur Anpassung benutzten Frequenzbereich ist, ist ihr Einfluss auf das azimuthale Mittel gering. Daher wird zur Anpassung

$$
S=\int_{q_{\min } \leq q \leq q_{\max }} \mathrm{d} \boldsymbol{q}^{2}\left|\ln [\|\widetilde{I}(\boldsymbol{q})\|-\mathcal{B}(q)]-\ln \frac{2}{\pi}-m_{1}-m_{2} \sqrt{q}-m_{3} q-m_{4} q^{2}\right|
$$

minimiert. Dies entspricht einer Anpassung von $\frac{2}{\pi} \mathcal{E}(q)$ an das azimuthale Mittel von $\|\widetilde{I}(\boldsymbol{q})\|$ $\mathcal{B}(q)$. Der Faktor $\frac{2}{\pi}$ entspricht gerade dem Mittelwert des Sinusbetrags. Zur Anpassung wurde die „Downhill Simplex“-Methode verwendet [107].

Ein Beispiel für eine vorgenommene Abschätzung an ein gemessenes Diffraktogramm ist in Abb. 4.5 zu sehen. Das Diffraktogramm in dieser Abbildung (s. Abb. 4.6b) wies nur einen schwachen Astigmatismus auf, d.h. es sind nahezu kreisförmige Thonringe zu finden, so dass eine azimuthale Mittelung möglich war, ohne die Oszillationen des Sinustermes aus Gl. (4.1) zu zerstören, d.h. $\chi_{\mathrm{s}}(\boldsymbol{q}) \approx \chi_{\mathrm{s}}(q)$.

Während die Anpassung des Hintergrundes recht optimal ist, gilt dies nicht unbedingt für die Einhüllende. Es hat sich allerdings gezeigt, dass die im nächsten Abschnitt beschriebene Anpassung des Defokus und des Astigmatismus an die Diffraktogramme nicht sehr sensitiv auf diese Abschätzung der Einhüllenden ist. Da die am Ende erreichte Genauigkeit bei der Bestimmung der Linsenfehler ausreicht, um sie innerhalb der Mikroskopauflösung zu korrigieren, 
wurde von einer weiteren Verbesserung dieser Anpassung abgesehen. Sollte eine höhere Genauigkeit benötigt werden, wäre diese Anpassung sicherlich ein guter Startpunkt für Verbesserungen. Mit der Einschränkung, die Zemlin-Tableaus nur von rein amorphen Probenbereichen aufzunehmen, ließe sich beispielsweise diese Anpassung wieder nach Mallick [105] vornehmen.

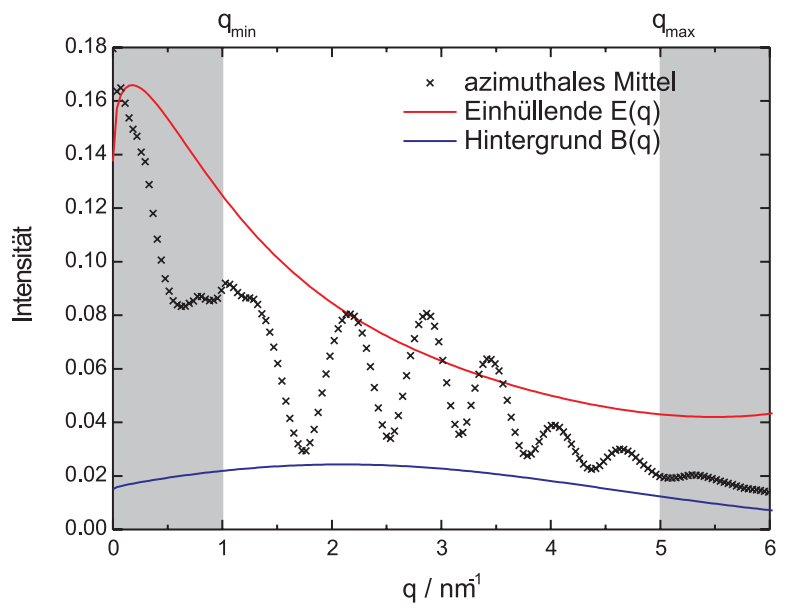

Abbildung 4.5: Abschätzungen für den Hintergrund $\mathcal{B}(q)$ und die Einhüllende $\mathcal{E}(q)$ für ein Diffraktogramm von amorphen Si $\left(D=-135 \mathrm{~nm}, C_{s}=0.49 \mathrm{~mm}, A_{2}=2.2 \mathrm{~nm}\right)$. Nur der unschattierte Bereich wurde für die Anpassung verwendet $\left(q_{\min }=1.0 \mathrm{~nm}^{-1}\right.$ und $\left.q_{\max }=5.0 \mathrm{~nm}^{-1}\right)$.

\subsubsection{Anpassung des Defokus und des Astigmatismus}

Nach Gleichung (4.1) gilt

$$
\left|\sin \left(-2 \pi \chi_{\mathrm{s}}(\boldsymbol{q})\right)\right|=\frac{\|\widetilde{I}(\boldsymbol{q})\|-\mathcal{N}(q)}{\mathcal{E}(q)},
$$

daher wird unter Veränderung der Werte für $A_{2 x}, A_{2 y}$ und $D$ nun versucht, den Sinusbetrag der Aberrationsfunktion an das entsprechend normierte Diffraktogramm zweidimensional anzupassen. Dabei wird die Größe

$$
S=\int_{q_{\min } \leq q \leq q_{\max }} \frac{\mathrm{d} \boldsymbol{q}^{2}}{q^{2}}|| \sin \left(-2 \pi \chi_{\mathrm{s}}(\boldsymbol{q})\right)\left|-\frac{\|\widetilde{I}(\boldsymbol{q})\|-\mathcal{N}(q)}{\mathcal{E}(q)}\right|
$$

mit dem Downhill-Simplex-Algorithmus [107] minimiert. In Abb. 4.6 ist das Ergebnis einer Anpassung gezeigt. Weitere Beispiele sind in Abb. $4.10 \mathrm{zu}$ sehen.

\subsubsection{Abschätzung der erreichten Genauigkeit}

Sicherlich hängt die Genauigkeit der einzelnen Anpassung von einer größeren Anzahl von Faktoren ab, wie z.B. dem mikroskopierten Material, der Größe der Verkippung des Strahls gegen 
(a)

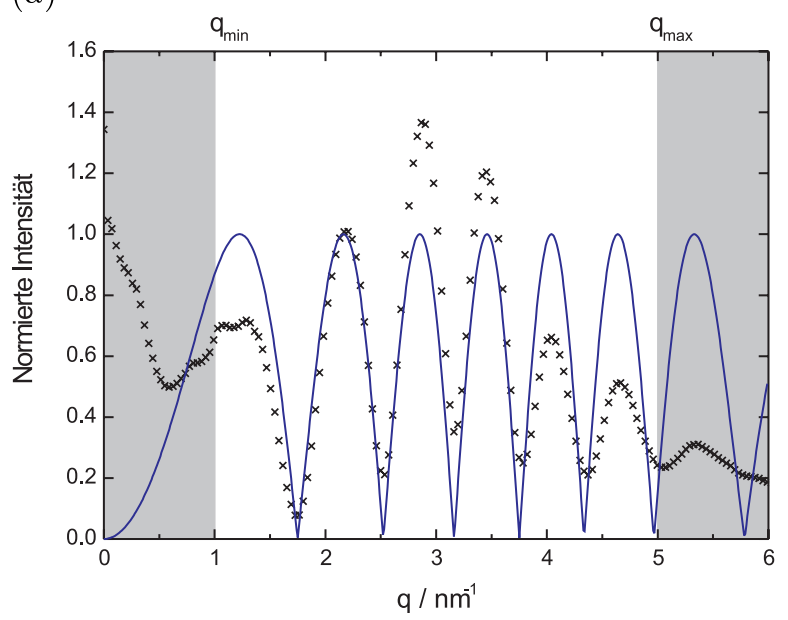

(b)

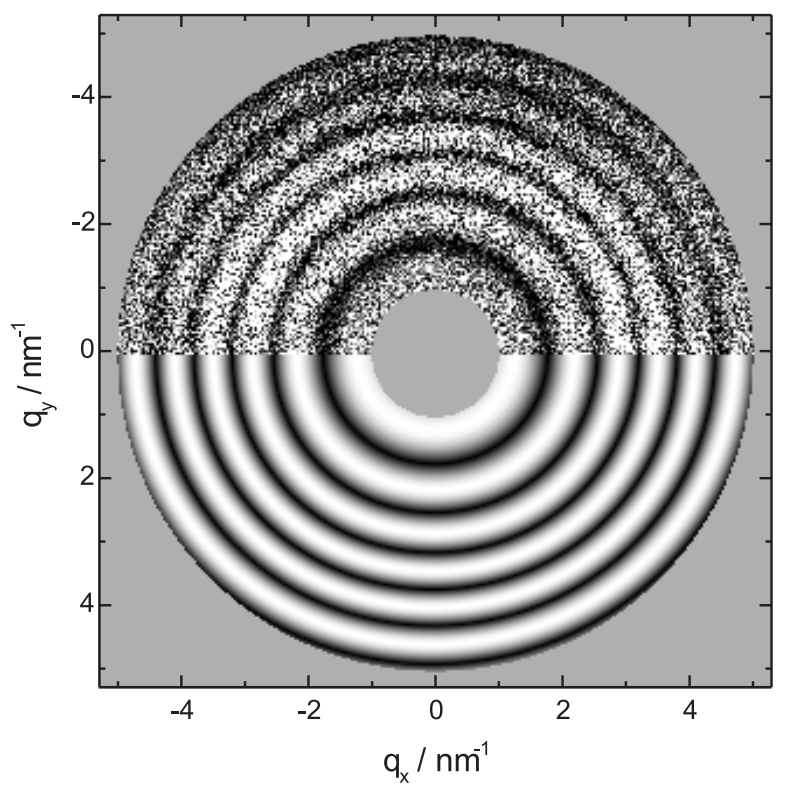

Abbildung 4.6: Anpassung der Aberrationsfunktion an ein Diffraktogramm von amorphen $\operatorname{Si}(D=$ $\left.-135 \mathrm{~nm}, C_{s}=0.49 \mathrm{~mm}, A_{2}=2.2 \mathrm{~nm}\right)$ für den Bereich von $1-5 \mathrm{~nm}^{-1}$. Abb. 4.5 zeigt die Anpassung von $\mathcal{B}(q)$ und $\mathcal{E}(q)$. (a) Vergleich des angepassten Sinusbetrags (durchgezogene Linie) mit dem entsprechend normierten azimuthalen Mittel $(\|\widetilde{I}(q)\|-\mathcal{B}(q)) / \mathcal{E}(q)$. Die Anpassung wurde nur im unschattierten Bereich vorgenommen. (b) Oben das normierte Diffraktogramm, unten der angepasste Sinusbetrag. 
die optische Achse oder auch vom Defokus und Astigmatismus selbst, da die Diffraktogramme sich mit diesen Parametern mehr oder weniger stark verändern. Es soll hier nun ein Fehler für Diffraktogramme, die unter typischen Bedingungen für Zemlin-Tableaus (s. Abschnitt 4.1) aufgenommen wurden, abgeschätzt werden. Dieser Fehler wird im Weiteren benutzt, um die ermittelten Effektivwerte für Defokus und 2-zähligen Astigmatismus bei der Bestimmung der axialen Aberrationskonstanten zu gewichten.

In experimentellen Tableaus wurde dazu jede einzelne Aufnahmen in ihre vier Quadranten zerlegt, und für diese nach der beschriebenen Methode Defokus und Astigmatismus bestimmt. Diese sollten im Prinzip für jeden Quadranten gleich sein, daher soll die Standardabweichung von ihrem Mittel als Abschätzung für den Fehler dienen. Abweichungen zwischen den Quadranten können entstehen durch z.B. eine schräge Probe (unterschiedliche Defokuswerte) oder dadurch, dass die isoplanatische Näherung nicht vollständig erfüllt wird. Diese Abweichungen würden allerdings auch bei der Bestimmung von Defokus und Astigmatismus über den gesamten Bildausschnitt zu einem Fehler in den Werten führen.

Es wurden zwei Tableaus mit einer Strahlkippung von $0.3^{\circ}$ und je acht Bildern untersucht. Ein Beispiel für ein gevierteiltes Bild ist in Abb. $4.7 \mathrm{zu}$ sehen. Die Abbildungen des einen Tableaus wurden von einem dünnen Bereich Klebstoff aufgenommen, stellvertretend für die Fälle, bei denen keine amorphe Probenstelle gefunden werden kann und daher auf den Klebstoff ausgewichen werden muss. Die Abbildungen des anderen Tableau wurden von amorphem $\mathrm{Si}$, stellvertretend für eine amorphe Probenstelle aufgenommen. Die Varianzen der einzelnen Defokuswerte bzw. der Größen des Astigmatismus wurden für jedes Tableau gemittelt. Damit ergaben sich die Abschätzungen für ihre Standardabweichungen:

\begin{tabular}{lcc} 
& $\sigma_{D}[\mathrm{~nm}]$ & $\sigma_{A_{2}}[\mathrm{~nm}]$ \\
\hline \hline Klebstoff & 1.26 & 1.25 \\
amorphes Si & 1.64 & 1.61 \\
\hline rms-Mittel & 1.46 & 1.44
\end{tabular}

\subsubsection{Berechnung der Aberrationskonstanten}

Aus den effektiven Werten für den Defokus und den 2-zähligen Astigmatismus des Tableaus lassen sich nun die Werte für die axialen Konstanten nach Gleichung (3.15) berechnen. Da das sich ergebene Gleichungssystem überbestimmt ist, sollen die Werte der Linsenfehler über eine $\chi^{2}$-Minimierung ermittelt werden.

Da die Aufnahmen im Tableau aufgrund von Probendrift und Verschiebung des Bildes (bedingt durch die Linsenfehler) nicht immer am gleichen Probenort durchgeführt werden, kann es zu einer Veränderung des Defokus über das Tableau kommen. Daher wird die Anpassung nur für die Effektivwerte des Astigmatismus vorgenommen. Die minimierte Größe lautet

$$
\begin{aligned}
\chi^{2} & =\sum_{n} \frac{\left[A_{2 x}^{(n)}-A_{2 x}^{\mathrm{eff}}\left(t_{x}^{(n)}, t_{y}^{(n)}, A_{3 x}, A_{3 y}, B_{x}, B_{y}, A_{2 x}\right)\right]^{2}}{\sigma_{A_{2}}^{2}+\left[\partial A_{2 x}^{\mathrm{eff}} / \partial t_{x}\right]^{2} \sigma_{t_{x}}^{2}+\left[\partial A_{2 x}^{\mathrm{eff}} / \partial t_{y}\right]^{2} \sigma_{t_{y}}^{2}} \\
& +\sum_{n} \frac{\left[A_{2 y}^{(n)}-A_{2 y}^{\mathrm{eff}}\left(t_{x}^{(n)}, t_{y}^{(n)}, A_{3 x}, A_{3 y}, B_{x}, B_{y}, A_{2 y}\right)\right]^{2}}{\sigma_{A_{2}}^{2}+\left[\partial A_{2 y}^{\mathrm{eff}} / \partial t_{x}\right]^{2} \sigma_{t_{x}}^{2}+\left[\partial A_{2 y}^{\mathrm{eff}} / \partial t_{y}\right]^{2} \sigma_{t_{y}}^{2}}
\end{aligned}
$$




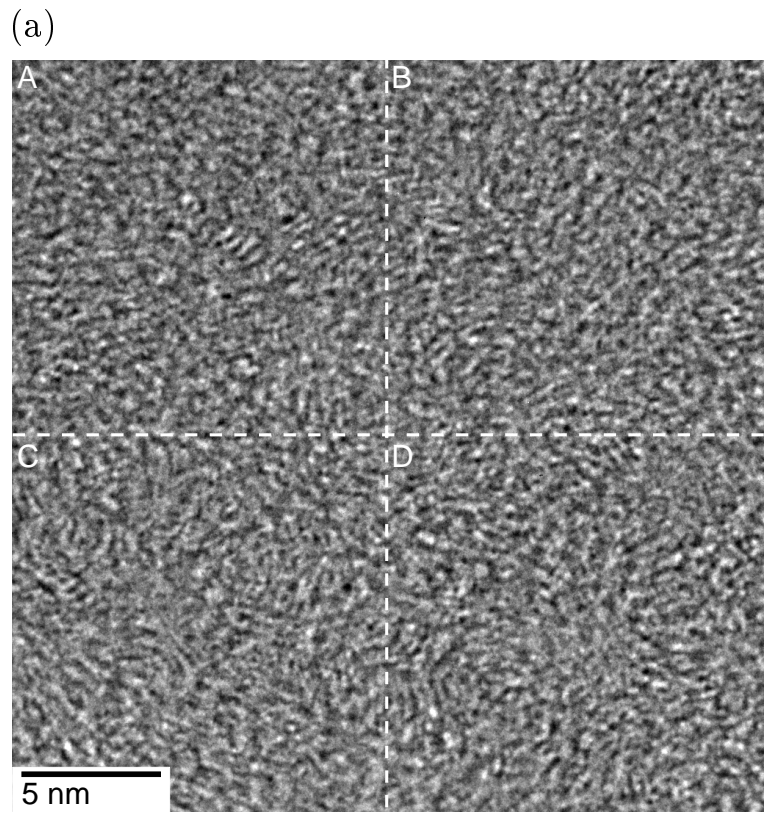

(b)
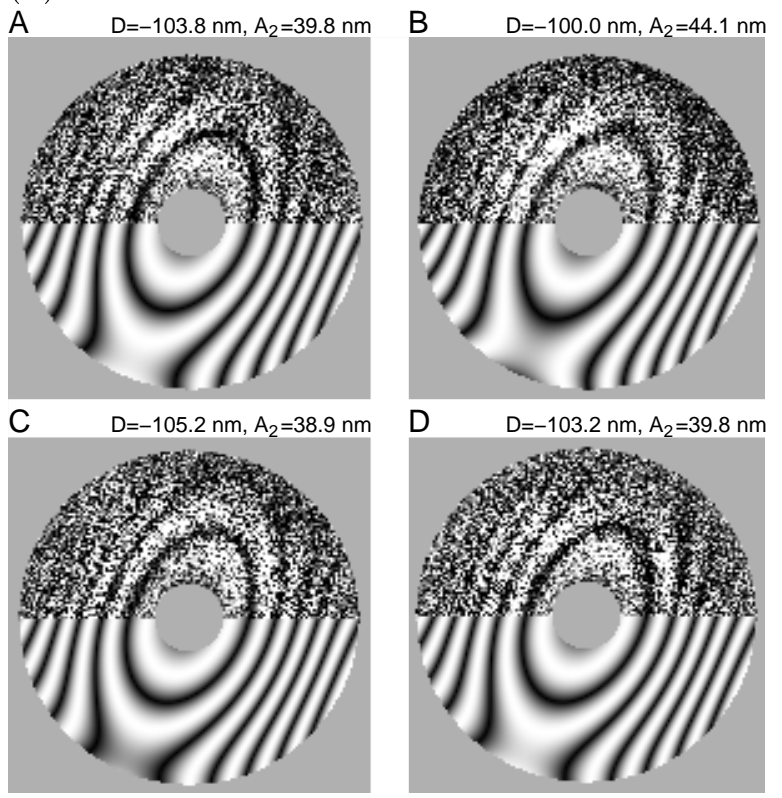

Abbildung 4.7: Zur Abschätzung der Fehler der Anpassung: (a) Die Aufnahme wurde in ihre vier Quadranten geteilt. (b) Anpassung der Quadranten. Die Veränderung des Defokus von unten links nach oben rechts beruht wahrscheinlich auf einer schrägen Probenrückseite. 
wobei der Index $n$ über alle Bilder des Tableaus läuft und die mit $n$ indizierten Größen die jeweiligen Verkippungswinkel und gemessenen Effektivwerte sind. Die Funktionen $A_{2 x}^{\text {eff }}$ und $A_{2 y}^{\text {eff }}$ aus Gleichung (3.15) geben den Zusammenhang zwischen den Effektivwerten und den axialen Konstanten an, wie in Abschnitt 3.4.2 beschrieben wurde. Der Fehler in den Verkippungswinkeln wird mittels gaußscher Fehlerfortpflanzung berücksichtigt. Er stammt aus der Kalibrierung der Strahlverkippung des Mikroskops (s. Anhang A). Die Anpassung wurde mittels der „Downhill Simplex“-Methode durchgeführt [107].

Um die Anpassung in der Nähe des Minimums zu starten, werden zunächst die Startwerte nach einer Idee von Thust et al. bestimmt [68]: Die axialen Konstanten lassen sich aus den Koeffizienten einer Fourierreihe über die Effektivwerte der 2-zählige Aberration bzgl. des Azimuthalwinkels der Strahlverkippung berechnen.

Die Standardabweichung der über Gl. (4.2) bestimmten Konstanten können mittels MonteCarlo Simulation bestimmt werden. Die Kippwinkel und die Effektivwerte für Defokus und 2zähligen Astigmatismus werden dabei zufällig innerhalb ihrer Fehler gaußverteilt variiert, und es wird angenommen, dass die Streuung der über die variierten Daten angepassten Konstanten der tatsächlichen Streuung entspricht [107].

\subsection{Vergleich zwischen rekonstruierter und simulierter Wellenfunktion}

Um die rekonstruierten Objektwellenfunktionen mit simulierten Wellenfunktionen qualitativ zu vergleichen, müssen die simulierten Wellenfunktionen noch an die Bedingungen der Rekonstruktion angepasst werden: Eine Apertur, die das begrenzte Übertragungsvermögen des Mikroskops berücksichtigt, muss eingefügt, die Wellenfunktionen müssen normiert und die bekannte Abweichung zwischen simulierter und gemessener Wellenfunktionen (Stobbs-Faktor) muss entsprechend berücksichtigt werden.

Die Simulationen berechnen auch Beiträge für hohe Raumfrequenzen, so berücksichtig z.B. die Multislice-Simulationen im verwendeten Softwarepaket EMS [108] standardmäßig Raumfrequenzen bis mind. $50 \mathrm{~nm}^{-1}$. Dies ist für eine korrekte Simulation der Vielfachstreuung notwendig. Das Mikroskop überträgt allerdings nur Frequenzen bis zum Informationslimit $q_{\text {Info }}$ (beim verwendeten Mikroskop $8.0 \mathrm{~nm}^{-1}$ ). Somit können auch nur Ojektwellen bis zu dieser Frequenz rekonstruiert werden. Daher werden in den simulierten Wellenfunktionen Bildanteile mit höherer Raumfrequenz mittels einer künstlichen Apertur entfernt. In Objektwellenrekonstruktionen von Defokusserien, die nicht unter optimalen Bedingungen aufgenommen wurden, oder bei denen die Abbildungsparameter nicht genau bestimmt werden konnten, kann auch in hohen Raumfrequenzen unterhalb des Informationslimits nur Rauschen enthalten sein. Hier erfordert ein Vergleich mit der Simulation evtl. eine kleinere Apertur.

Zum Vergleich werden zunächst beide Funktionen normiert, so dass ihr Mittelwert in beiden Fällen 1 beträgt. Für den qualitativen Vergleich reicht eine solche Normierung aus. Eine korrekte Normierung wäre erreichbar, wenn sich in der rekonstruierten Wellenfunktion auch ein Bereich ohne Probe, d.h. Vakuum, befände. Der komplexe Wert der Wellenfunktion in diesem Bereich könnte mit der einfallenden Welle in der Simulation verglichen werden. Fehlt so ein Bereich, so ist es nur möglich, die Amplitude und nicht die Phase dieses Wertes aus weiteren 
experimentellen Abbildungen zu erlangen, da der Abbildungsprozess (Intensitätsbildung) nur bis auf eine globale Phase eindeutig ist.

Ohne weitere Berücksichtigung des Stobbs-Faktors, treten große Unterschiede in rekonstruierten und simulierten Wellenfunktionen auf. Diese Unterschiede werden in Abb. 4.8a am Beispiel einer $\mathrm{Si}\langle 110\rangle$-Zonenachse (s. auch Abb. 4.9) verdeutlicht. In der Abbildung sind die Werte der einzelnen „Pixel“ aus einer Einheitszelle der mittels Multislice-Methode simulierten Wellenfunktion für verschiedene Dicken $t$, sowie aus einem etwa zwei Einheitszellen großen Bereich der rekonstruierten (und linsenfehlerkorrigierten) Wellenfunktion auf der komplexen Zahlenebene gezeigt. Die Amplitude eines Punktes entspricht seinem Abstand vom Ursprung, seine Phase dem Winkel zwischen der Verbindungslinie des Punktes zum Ursprung und der reellen Achse. Es ist augenfällig, dass die Streuung (entspricht dem Kontrast) der Amplituden und Phasen in der rekonstruierten Wellenfunktion klein gegenüber den Streuungen der simulierten Wellenfunktion ist. Insbesondere fällt dies bei den Phasen auf. Diese streuen für $t=10.4 \mathrm{~nm}$ über einen Winkel von $2 \pi$, während sie für die Rekonstruktion innerhalb eines Winkels von $\pi / 4$ bleiben.

Um diese Abweichungen heuristisch zu berücksichtigen, wird zur simulierten Wellenfunktion ein reeller Hintergrundwert $H$ (üblicherweise 3) addiert. Diese Addition entspricht in Diagramm 4.8a einer Verschiebung der Punkte aus den simulierten Wellenfunktionen um $H$ in Richtung der positiven reellen Achse. Eine anschließende Normierung der verschobenen simulierten Wellenfunktionen auf den Mittelwert 1 ergibt die in Abb. 4.8b gezeigte Situation. Die noch vorhandenen Unterschiede in den Streuungen der Phase und Amplitude sind bei qualitativen Vergleichen der Wellenfunkionen nicht mehr sichtbar, sie könnten ansonsten durch die Wahl eines größeren Wertes von $H$ beseitigt werden.

Wie in Abschnitt 3.3 erläutert, werden bei Simulationen die Effekte der thermisch diffusen und inelastischen Streuung nur durch einen Absorptionsfaktor berücksichtigt. Diese beiden Effekte tragen allerdings zu einem Intensitätshintergrund in der Abbildung bei. Mit der hier skizzierten Vorgehensweise wird dieser Hintergrund als konstant angenommen und auf die simulierte Wellenfunktion addiert. Da die inelastisch gestreuten Anteile in sehr kleine Winkel gestreut und diese Anteile aufgrund des Energieverlustes der Elektronen auch nur noch unscharf durch die Objektivlinse fokussiert werden, ist die Annahme eines konstanten Hintergrundes für diese Anteile sicherlich gerechtfertigt. Aufwendige Simulationen haben gezeigt, dass die thermisch diffus gestreuten Anteile durchaus noch eine Struktur zeigen, allerdings ist ihr Kontrast um den Faktor 2-6 geringer als der Kontrast der elastisch gestreuten Anteile [109]. Daher werden sie hier auch als kontrastlos angenommen, was für den qualitativen Bildvergleich ausreichend ist.

\subsection{Kontrollmessung}

Um zu kontrollieren, ob die Objektwellenrekonstruktion zusammen mit der Korrektur der Linsenfehler funktioniert, wurde die Objektwelle von Si in [110]-Projektion rekonstruiert. In Abbildung 4.9 ist ein Ausschnitt aus dieser Defokusserienrekonstruktion zu sehen, vor und nach Korrektur der Linsenfehler (s.u.).

Diese Projektion wurde für eine Kontrolle gewählt, da sie ein charakteristisches Merkmal bei einer hohen Raumfrequenz $\left(7.37 \mathrm{~nm}^{-1}\right)$, aber innerhalb des Informationslimit des benutzten 
(a)

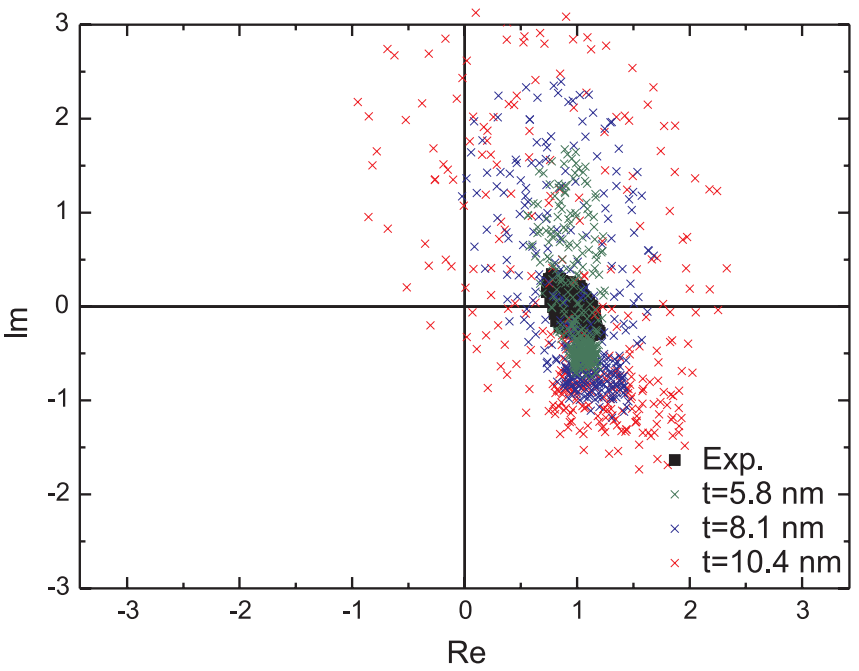

(b)

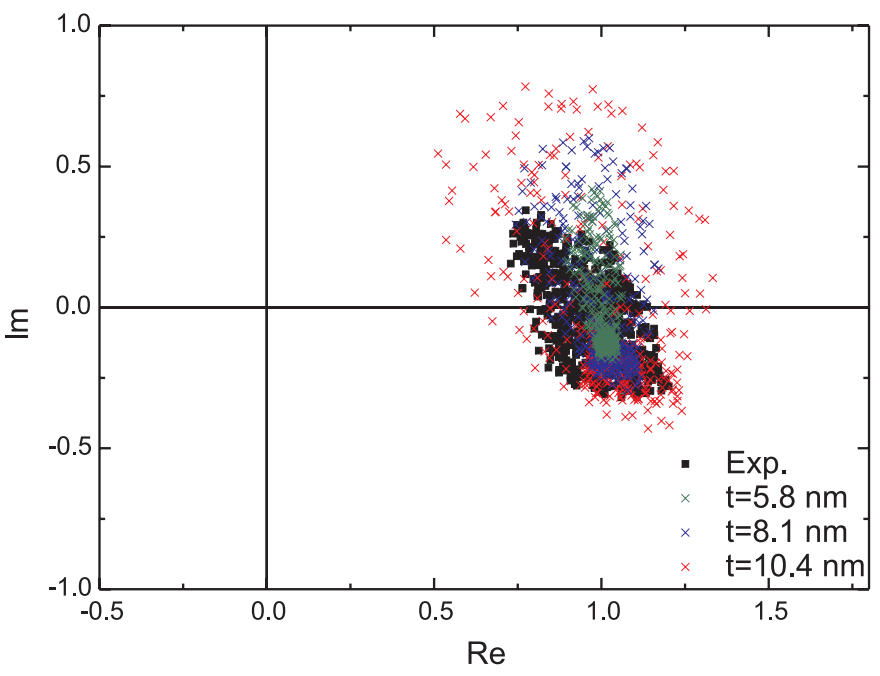

Abbildung 4.8: Auftragung von Real- und Imaginärteil von normierten Wellenfunktionen von $\operatorname{Si}\langle 110\rangle$. Gezeigt sind die rekonstruierte Wellenfunktionen (mit Exp. gekennzeichnet) und simulierte Wellenfunktionen für verschiedene Probendicken $t$. (a) Ohne Berücksichtigung des Stobbsfaktors. (b) Nach Addition eines Hintergrundes $H=3$ und anschließender Normierung. 
Phase (unkorrigiert)

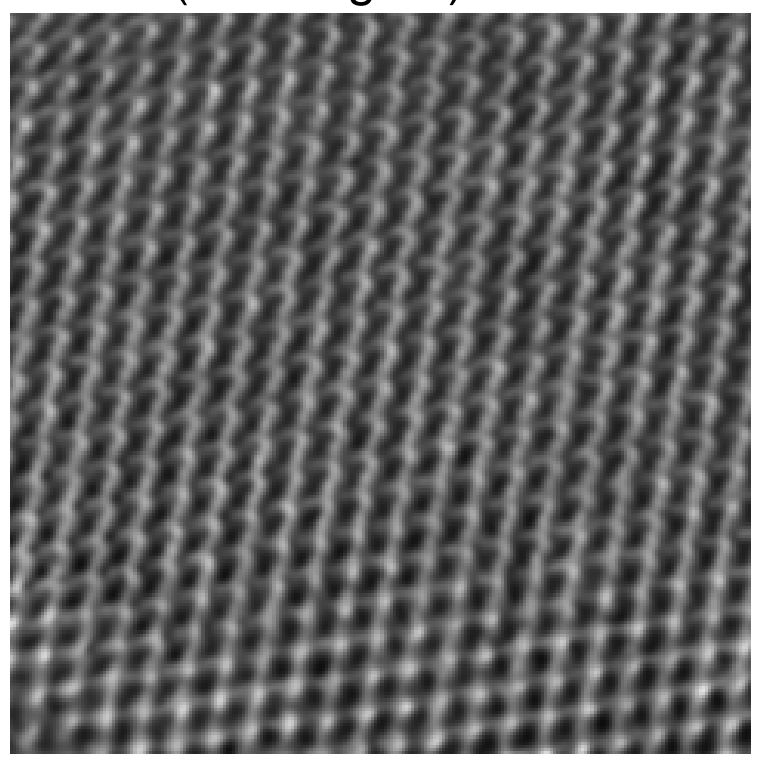

Phase (korrigiert)

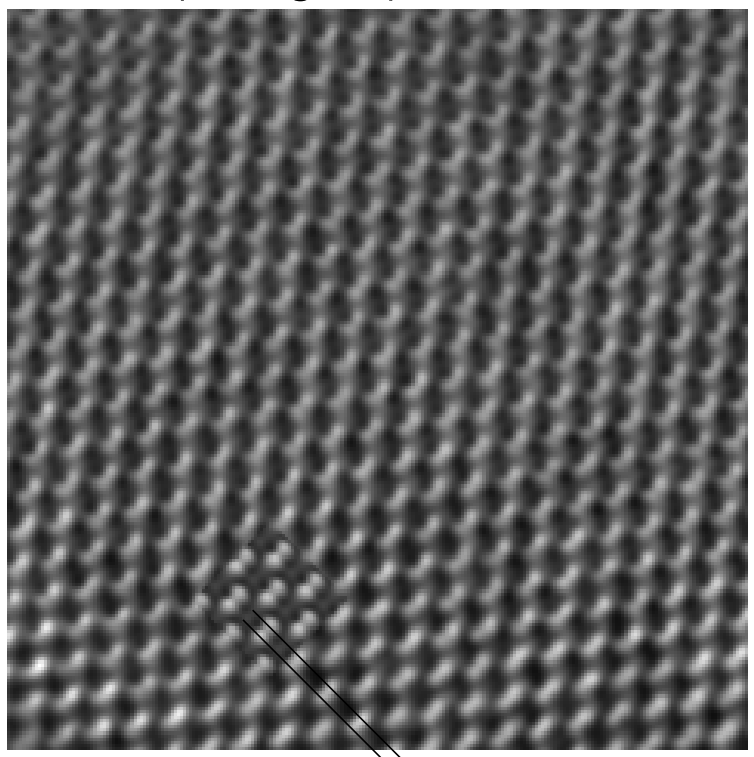

$0.136 \mathrm{~nm}$
Amplitude (unkorrigiert)

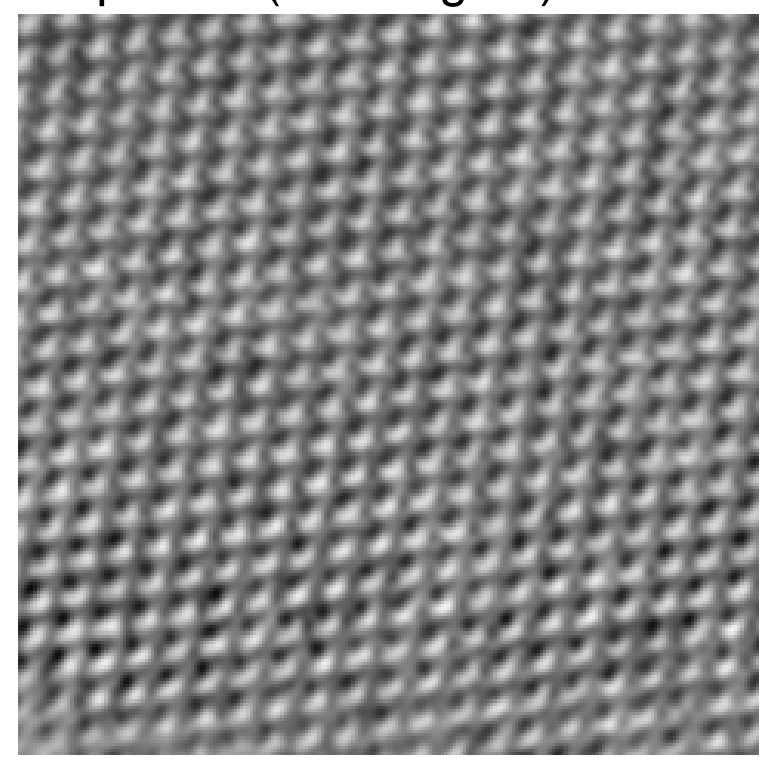

Amplitude (korrigiert)

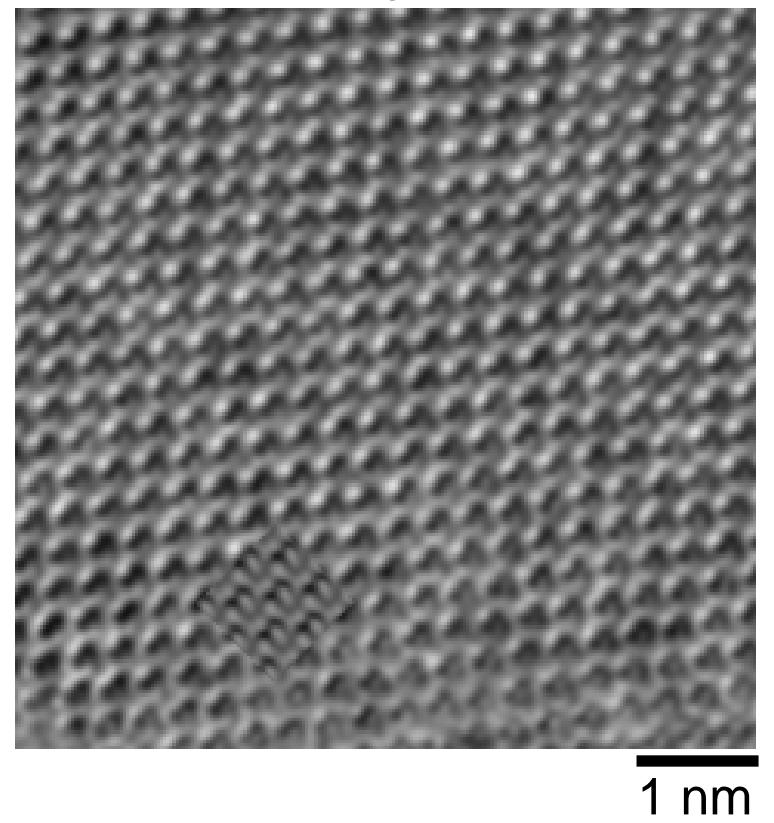

Abbildung 4.9: Phase und Amplitude der rekonstruierten Objektwelle vor Korrektur und nach Korrektur der residualen Aberrationen. In den korrigierten Wellenfunktionen ist eine simulierte Wellenfunktion eingefügt. Der Abstand der „dumbbells“ ist in der rekonstruierten Phase noch einmal gesondert eingezeichnet. 
Mikroskops besitzt, das bei hinreichender Korrektur der Linsenfehler direkt sichtbar werden sollte: Die beiden einander nächsten Atomsäulen, die sog. „dumbbells“, haben einen Abstand von $a / 4=0.136 \mathrm{~nm}$. Ihr Abstand ist nochmal gesondert in Abb. 4.9 eingezeichnet.

In Abbildung 4.10 ist ein kurz vor der Defokusserie aufgenommenes Zemlintableau zu sehen, aus dem die Linsenfehler bestimmt wurden. Die Strahlverkippung gegen die optische Achse im Tableau betrug $0.3^{\circ}$. Die Anpassung der Diffraktogramme erfolgte für Raumfrequenzen zwischen $q_{\min }=1.0 \mathrm{~nm}^{-1}$ und $q_{\max }=4.5 \mathrm{~nm}^{-1}$. Die gemessenen Aberrationskonstanten sind in der folgenden Tabelle aufgeführt. Die Bestimmung ergab einen $\chi^{2}$-Wert von 23.4 bei 10 Freiheitsgraden. Der residuale Defokus wurde mit einem anderen Verfahren bestimmt: In der Defokusserienrekonstruktion befand sich ein amorphes Randstück (direkt unter dem hier gezeigten Auschnitt). Der Amplitudenkontrast dieses Ausschnitts wurde durch Variation des Defokuswertes minimiert.

\begin{tabular}{ccc} 
Aberrationskonstante & Wert [nm] & Stdabw. [nm] \\
\hline \hline$D$ & -3.125 & \\
$A_{2 x}$ & 2.04 & 0.52 \\
$A_{2 y}$ & 2.73 & 0.50 \\
$A_{3 x}$ & -266 & 46 \\
$A_{3 y}$ & -196 & 47 \\
$B_{x}$ & 229 & 47 \\
$B_{y}$ & 2033 & 47 \\
\hline
\end{tabular}

Die simulierte Welle wurde für eine Probendicke von $10.4 \mathrm{~nm}$ mit einer Strahlverkippung von $8 \mathrm{mrad}$ in (111)-Richtung und einem Hintergrundswert $H$ von 3 berechnet. Der Vergleich mit Simulationen ergibt, dass die Dicke der Si-Schicht vom unteren zum oberen Bildrand hin leicht zunimmt. So ist zu erkennen, dass die Amplitude der in Abb. 4.9 eingefügten simulierten Wellenfunktion im unteren Bereich der Rekonstruktion größere Ähnlichkeit zur rekonstruierten Amplitude hat als im oberen Bereich.

Ein Vergleich zwischen der unkorrigierten und der aberrationskorrigierten Wellenfunktion zeigt, dass erst die Korrektur eine direkte Interpretation der Wellenfunktion ermöglicht. Obwohl die Unsicherheit in der Größe der residualen Aberrationen nicht ausreicht, um nach der Abschätzung aus Abschnitt 4.3.1 bis zum Informationslimit eine direkt interpretierbare Welle zu gewährleisten, reicht sie doch für entsprechende Korrekturen bis kurz vor das Limit $\left(\approx 7.5 \mathrm{~nm}^{-1}\right)$ aus, was sich auch in der Sichtbarkeit der ,dumbbells“ widerspiegelt. 

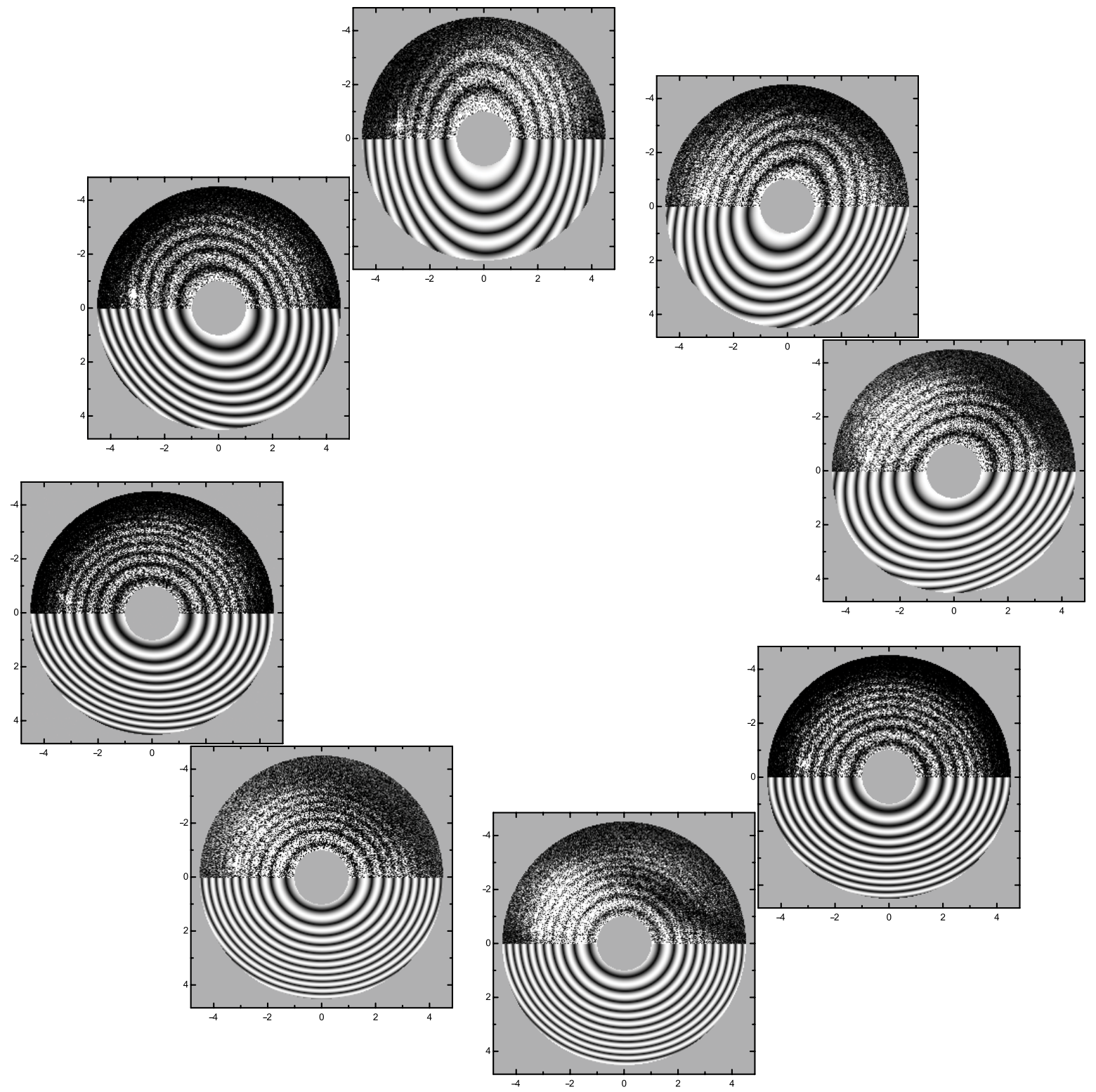

Abbildung 4.10: Zemlin-Tableau. Die Abbildungen sind mit einer Strahlverkippung von $0.3^{\circ}$ aufgenommen worden. Die obere Hälfte zeigt das Diffraktogramm, die untere die Anpassung dieser Aufnahmen für Raumfrequenzen zwischen $1.0 \mathrm{~nm}^{-1}$ und $4.5 \mathrm{~nm}^{-1}$. 


\section{Ausgedehnte Defekte in GaN}

Mit der Objektwellenrekonstruktion ist es möglich, ausgedehnte Defekte ohne störende Effekte der optischen Delokalisierung zu beobachten. Eine Technik, um die Verschiebungsfelder $\boldsymbol{u}(\boldsymbol{r})$ dieser Defekte zu messen, ist die „Geometric Phase Analyis“ (GPA) [110, 111], die in Abschnitt 5.1 ausführlicher vorgestellt wird. Aus den Ableitungen dieser Verschiebungsfelder lassen sich anschließend die Verzerrungsfelder bestimmen.

Bei konventioneller HRTEM lässt sich die GPA-Technik nur unter ganz bestimmten Abbildungsbedingungen einsetzen, bei denen die Effekte der optischen Delokalisierung die Messung nicht verfälschen [112]. In dieser Arbeit wird die GPA erstmals auf komplexe Objektwellenfunktionen angewendet. Dabei entfallen die Einschränkungen bzgl. der Abbildungsbedingungen, und die GPA kann vielseitiger eingesetzt werden.

Im Abschnitt 5.2 wird eine Übersicht über die in dieser Arbeit untersuchten GaN-Proben und die verwendeten Messgeometrien gegeben. In den Abschnitten 5.3 bis 5.8 werden die verschiedenen ausgedehnten Defekte, wie Stapelfehler, Korngrenzen, Partialversetzungen sowie Reaktionen zwischen Versetzungen und Stapelfehler, wie sie in diesen Proben gefunden wurden, analysiert.

\subsection{Geometric Phase Analysis}

\subsubsection{Grundidee}

Die hier skizzierte Idee der GPA folgt in der Argumentation den Ausführungen von Hÿtch [110, 111]. Während die Formulierungen von Hÿtch für die Intensitäten in einer Abbildung gelten, werden sie hier auf (komplexe) Objektwellenfunktionen ausgedehnt.

Die Objektwelle eines perfekten Kristalls lässt sich als Summe ebener Wellen schreiben

$$
\psi(\boldsymbol{r})=\sum_{\boldsymbol{g}} C_{\boldsymbol{g}} \exp \{\mathrm{i} 2 \pi \boldsymbol{g} \cdot \boldsymbol{r}\},
$$

wobei die $\boldsymbol{g}$ den Bragg-Reflexen des Kristalls entsprechen und die (komplexen) $C_{\boldsymbol{g}}$ die zugehörigen Fourierkomponenten sind. Abweichungen vom perfekten Kristall lassen sich durch eine Ortabhängigkeit von $C_{\boldsymbol{g}}$ beschreiben, d.h. mit der „lokalen“ Fourierkomponente $C_{\boldsymbol{g}}(\boldsymbol{r})$ :

$$
\psi(\boldsymbol{r})=\sum_{\boldsymbol{g}} C_{\boldsymbol{g}}(\boldsymbol{r}) \exp \{\mathrm{i} 2 \pi \boldsymbol{g} \cdot \boldsymbol{r}\} .
$$

Eine Definition der $C_{\boldsymbol{g}}(\boldsymbol{r})$ ergibt sich aus der Fouriertransformation der Gleichung (5.2):

$$
\widetilde{\psi}(\boldsymbol{k})=\sum_{\boldsymbol{g}} \widetilde{C}_{\boldsymbol{g}}(\boldsymbol{k}) \otimes \delta(\boldsymbol{k}-\boldsymbol{g}) .
$$


Diese Fouriertransformation entspricht einer Faltung der $\widetilde{C}_{\boldsymbol{g}}(\boldsymbol{k})$ mit den reziproken Gitterpunkten $\boldsymbol{g}$. Sind die $\widetilde{C}_{\boldsymbol{g}}(\boldsymbol{k})$ nun nur für die 1. Brillouinzone definiert, bzw. 0 für $\boldsymbol{k}$ außerhalb, ergibt sich eine vollständige, nicht überlappende Abdeckung der Fourierebene.

In der Darstellung der Komponenten $C_{\boldsymbol{g}}(\boldsymbol{r})=A_{\boldsymbol{g}}(\boldsymbol{r}) \exp \left\{\mathrm{i} \varphi_{\boldsymbol{g}}(\boldsymbol{r})\right\}$ in Form von Amplitude $A_{\boldsymbol{g}}(\boldsymbol{r})$ und Phase $\varphi_{\boldsymbol{g}}(\boldsymbol{r})$ wird aus Gl. (5.2)

$$
\psi(\boldsymbol{r})=\sum_{\boldsymbol{g}} A_{\boldsymbol{g}}(\boldsymbol{r}) \exp \left\{\mathrm{i}\left[2 \pi \boldsymbol{g} \cdot \boldsymbol{r}+\varphi_{\boldsymbol{g}}(\boldsymbol{r})\right]\right\} .
$$

Wird ein perfekter Kristall um einen Vektor $\boldsymbol{u}$ verschoben $\boldsymbol{r} \rightarrow \boldsymbol{r}-\boldsymbol{u}$, so wird Gleichung (5.1) $\mathrm{zu}$

$$
\psi(\boldsymbol{r})=\sum_{\boldsymbol{g}} A_{\boldsymbol{g}} \exp \left\{\mathrm{i}\left[2 \pi \boldsymbol{g} \cdot \boldsymbol{r}-2 \pi \boldsymbol{g} \cdot \boldsymbol{u}+\varphi_{\boldsymbol{g}}\right]\right\} .
$$

Für einen konstanten Vektor $\boldsymbol{u}$ ist diese Gleichung exakt, für langsam veränderliche Verschiebungsfelder lässt sich wiederum ein ortsabhängiges $\boldsymbol{u}(\boldsymbol{r})$ einführen. Dieses Vorgehen entspricht der Säulennäherung der konventionellen TEM [57]. Ein Vergleich von Gl. (5.4) mit Gl. (5.5) liefert dann bis auf einen beliebigen konstanten Phasenfaktor $\varphi_{g}$ :

$$
\varphi_{\boldsymbol{g}}(\boldsymbol{r})=-2 \pi \boldsymbol{g} \cdot \boldsymbol{u}(\boldsymbol{r}) .
$$

Das bedeutet, dass sich aus den Phasen $\varphi_{\boldsymbol{g}}(\boldsymbol{r})$ der lokalen Fourierkomponenten $C_{\boldsymbol{g}}(\boldsymbol{r})$ für einen beliebigen Reflex $\boldsymbol{g}$ das Verschiebungsfeld bis auf Vielfache des zu $\boldsymbol{g}$ gehörigen Ebenenabstandes in Richtung $\boldsymbol{g}$ messen lässt. Aus verschiedenen Reflexen lässt sich daraus das vektorielle Verschiebungsfeld $\boldsymbol{u}(\boldsymbol{r})$ ermitteln, zumindestens senkrecht zur Zonenachse.

Um die Phase $\varphi_{\boldsymbol{g}}(\boldsymbol{r})$ nicht mit der Phase der Objektwelle zu verwechseln, wird sie als geometrische Phase bezeichnet.

\subsubsection{Messung der Verschiebungsfelder}

Eine Vorschrift, $C_{\boldsymbol{g}}(\boldsymbol{r})$ aus einer rekonstruierten Objektwellen $\psi(\boldsymbol{r})$ zu berechnen, ist bereits durch Gleichung (5.3) gegeben: Die Fouriertransformation der Objektwelle $\psi(\boldsymbol{k})$ wird um den Vektor $\boldsymbol{g}$ verschoben, $\boldsymbol{k} \rightarrow \boldsymbol{k}-\boldsymbol{g}$, und die Anteile außerhalb der 1. Brillouinzone werden 0 gesetzt. Eine Rücktransformation liefert $C_{\boldsymbol{g}}(\boldsymbol{r})$ für diesen Reflex. In der Praxis wird eine Gaußfunktion exp $\left\{-k^{2} / k_{0}^{2}\right\}$ zum Maskieren der Anteile in der Nähe des Reflexes benutzt, da die Anteile der Wellenfunktion für weiter vom zugehörigen Reflex $\boldsymbol{g}$ entfernte $\boldsymbol{k}$ ein schlechtes Signal-Rausch-Verhältnis haben. Auch bietet es sich an, starke Reflexe zu verwenden, um möglichst rauschfreie geometrische Phasen zu erhalten.

Für die Anwendungen auf GaN in dieser Arbeit wurden immer die zueinander senkrechten Reflexe (0002), zugehöriger Ebenenabstand $\frac{1}{2}[0001]$, und (1100), zugehöriger Ebenenabstand $\frac{1}{2}[1 \overline{1} 00]$, benutzt, sowie $k_{0}=0.75 \mathrm{~nm}^{-1}$. Dabei werden die genauen reziproken Vektoren $\boldsymbol{g}$ der Reflexe aus einem Diffraktogramm der untersuchten Objektwelle mittels eines Schwerpunktverfahrens [113] auf Subpixel-Niveau bestimmt. Somit wird das mittlere Gitter in der Welle als Referenzgitter zur Messung der Verschiebungsfelder verwendet. Auch die oben beschriebene Verschiebung der Fouriertransformation wird auf Subpixel-Niveau durchgeführt, indem dieser Schritt entsprechend des Verschiebungssatzes der Fouriertransformation im Realraum durchgeführt wird. 
Die konstante additive Phase $\varphi_{\boldsymbol{g}}$ in den geometrischen Phasen kann ignoriert werden, denn es werden keine absoluten Werte dieser Phase verwendet. Die Verzerrungsfelder $\epsilon_{i j}$ und Rotation $\omega_{x y}$ werden aus der Ableitung des Verschiebungsfeldes berechnet:

$$
\begin{aligned}
& \epsilon_{x x}=\frac{\partial u_{x}}{\partial x}, \quad \epsilon_{x y}=\frac{1}{2}\left\{\frac{\partial u_{x}}{\partial y}+\frac{\partial u_{y}}{\partial x}\right\}, \\
& \epsilon_{y y}=\frac{\partial u_{y}}{\partial y}, \quad \omega_{x y}=\frac{1}{2}\left\{\frac{\partial u_{x}}{\partial y}-\frac{\partial u_{y}}{\partial x}\right\} .
\end{aligned}
$$

\subsubsection{Fehlerquellen}

Die GPA misst die Verschiebungsfelder aus der Objektwelle, bzw. in der ursprünglichen Formulierung aus der Abbildung selbst. Diese ,scheinbaren“ Verschiebungen müssen nicht immer den tatsächlichen Verschiebungen der Atome aus ihrer nominellen Lage entsprechen.

Wenn die GPA auf HRTEM-Abbildungen durchgeführt wird, ergeben sich die größten Abweichungen durch die optische Delokalisierung, so dass die GPA nur unter bestimmten Abbildungsbedingungen auszuführen ist. Gerade die Effekte von Probenverkippung und Änderungen der Probendicke werden in nichtzentrosymmetrischen Strukturen durch die Delokalisierung problematisch [112]. Aufgrund der Verwendung von rekonstruierten Wellenfunktionen entfällt diese Fehlerquelle.

An Orten mit starken Variationen in der Probendicke und in der Probenverkippung, wie z.B. im Kern einer Versetzung, können noch Abweichungen zwischen den Verschiebungen in der Objektwelle und den Verschiebungen in der Struktur selbst auftreten, die zu weiteren Fehlern in der GPA führen [12]. Inwieweit diese starken Variationen die mittels GPA gemessenen Verzerrungsfelder verfälschen, ist noch nicht untersucht. Auch ist nicht klar, welche Bedeutung Größen wie $C_{\boldsymbol{g}}(\boldsymbol{r})$, die aus der Annahme eines perfekten Kristalls stammen, noch im Bereich von ausgedehnten Defekten haben. So hat z.B. ein Kristallreflex $\boldsymbol{g}$ im Kern einer Versetzung keine sinnvolle Bedeutung mehr. Aus diesen Gründen sind die Ergebnisse der GPA in Bereichen wie Versetzungskernen nicht interpretierbar.

\subsection{Wachstum und Geometrie der Proben}

Die in dieser Arbeit verwendeten Proben wurden mit Plasma-unterstützter Molekularstrahlepitaxie (MBE) von der MBE-Gruppe des IV. Physikalischen Institutes der Universität Göttingen gewachsen. Details zu diesem Wachstumsprozess können in [43] gefunden werden. Die Substrattemperatur beim Wachstum der GaN:Mn-Schichten betrug $650^{\circ} \mathrm{C}$, das StickstoffAngebot wurde für alle Proben konstant (Gasfluss: $1 \mathrm{sccm}$, Leistung der eingestrahlten Mikrowellen: 450W) gehalten. Um erfolgreich Mn in Anteilen von bis zu 5 at\% in die Proben einzubauen, musste das $\mathrm{GaN}$ bei diesen niedrigen Temperaturen (optimale Temperatur: $750^{\circ} \mathrm{C}$ ) und in einem stickstoffreichen Wachstumsregime gewachsen werden. Bereits die niedrigen Temperaturen als auch das Wachstum im N-reichen Regime führen im Vergleich zu GaN, das bei optimalen Bedingungen gewachsen wurde, zu schlechterer Kristallqualität. In Abb. 5.1 sind die Ga- und Mn-Flüsse gemessen als „Beam-Equivalent-Pressure“ (BEP) für die in dieser Arbeit untersuchten Proben gezeigt, normiert auf den Ga-Fluss beim stöchiometrischen Verhältnis. Das stöchiometrische Verhältnis bei dieser Wachstumtstemperatur wird für ein Ga-BEP von 


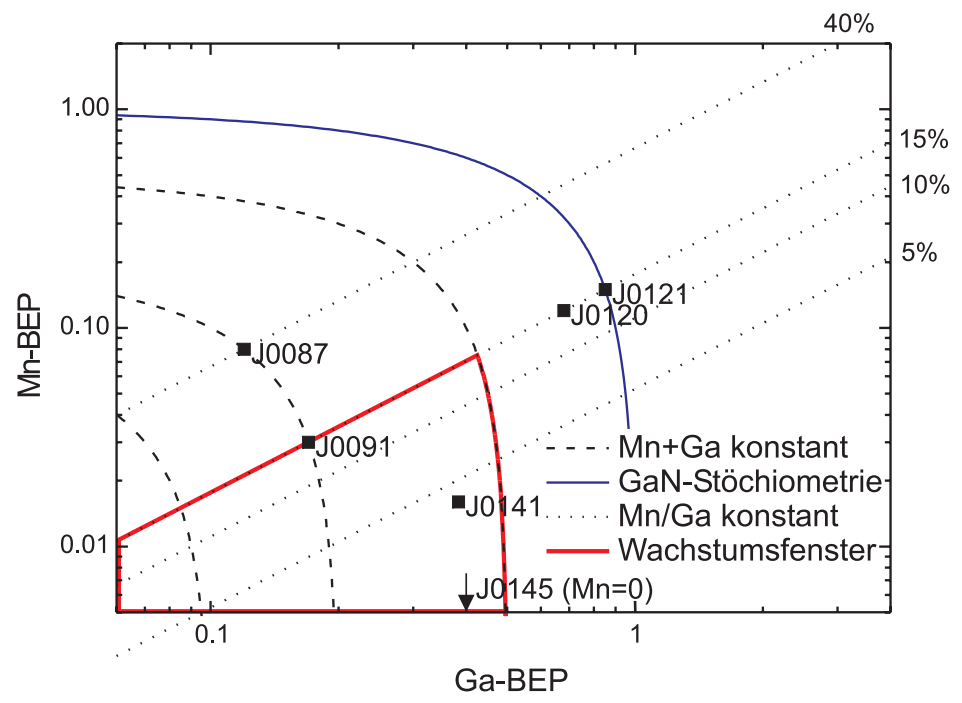

Abbildung 5.1: Wachstumsdiagramm für die in dieser Arbeit untersuchten Proben. Die Ga- und MnFlüsse sind auf den Ga-Fluss normiert, der der Stöchiometrie entspricht. Die gestrichelten Linien zeigen einen konstanten Metallfluss, die gepunkteten Linien ein konstantes Mn/Ga-Angebot. Der Bereich, in dem Mn ohne die Bildung von Ausscheidungen eingebaut werden kann, ist zusätzlich eingezeichnet (nach [43]).

$0.5 \cdot 10^{-7}$ mbar erreicht. Probe J0145 wurde bei gleichem Gesamtmetall-Fluss wie Probe J0141 gewachsen, allerdings ohne Mn.

Bei allen Proben wurde ein Si[111]-Substrat verwendet. Bei den Proben J0087, J0091, J0120 und J0121 wurde zunächst in der MBE eine $70 \mathrm{~nm}$ AlN-Pufferschicht bei höheren Temperaturen aufgewachsen. Auf dieser Schicht wurde dann die GaN:Mn-Schicht deponiert (Abb. 5.2a). Die GaN:Mn-Schichten der Proben J0141 und J0145 wurden hingegen auf einem mittels MOCVD hergestellten GaN-Template von etwa $150 \mathrm{~nm}$ Dicke aufgebracht. Auch hier wurde zwischen Si-Substrat und der GaN-Templateschicht zunächst eine $250 \mathrm{~nm}$ dicke AlNPufferschicht gewachsen. (Abb. 5.2b)

(a)

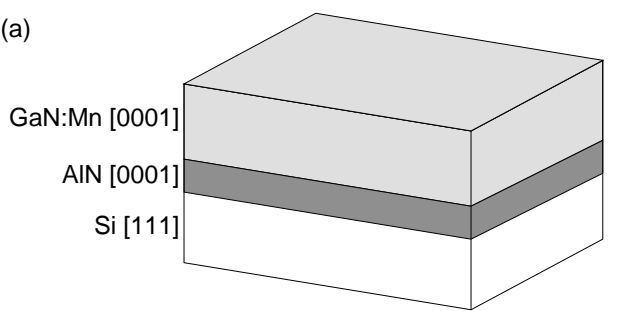

(b)

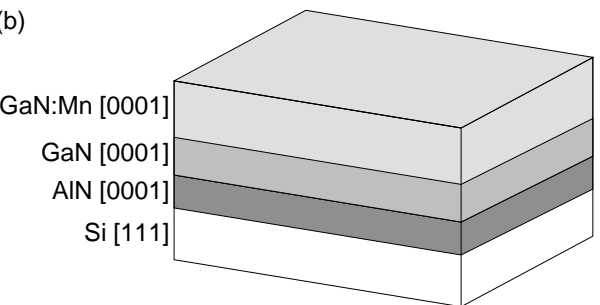

Abbildung 5.2: Schematischer Schichtaufbau: (a) Proben J0087, J0091, J0120 und J0121. Die Richtung [0001] ist die Wachstumsrichtung. Auf das Si-Substrat wurde zunächst eine AlN-Pufferschicht aufgebracht, auf der die Mn-dotierte GaN-Schicht gewachsen wurde. (b) Proben J0141 und J0145. Unterhalb der MBEgewachsenen Mn-dotierten GaN-Schicht befindet sich ein undotiertes GaN-Template, das mit MOCVD auf dem mit einer AlN-Pufferschicht bedeckten Si-Substrat hergestellt worden ist.

Zur Untersuchung der Proben im TEM wurden zwei verschiedene Messgeometrien verwendet. Der vorwiegende Teil der Untersuchungen wurde in Querschnittsgeometrie durchgeführt. Die 
Präparation der Aufsichtsproben ist aufwendiger und wird detailliert in Anhang D beschrieben. In Abb. 5.3 sind die beiden Geometrien noch einmal schematisch verdeutlicht.

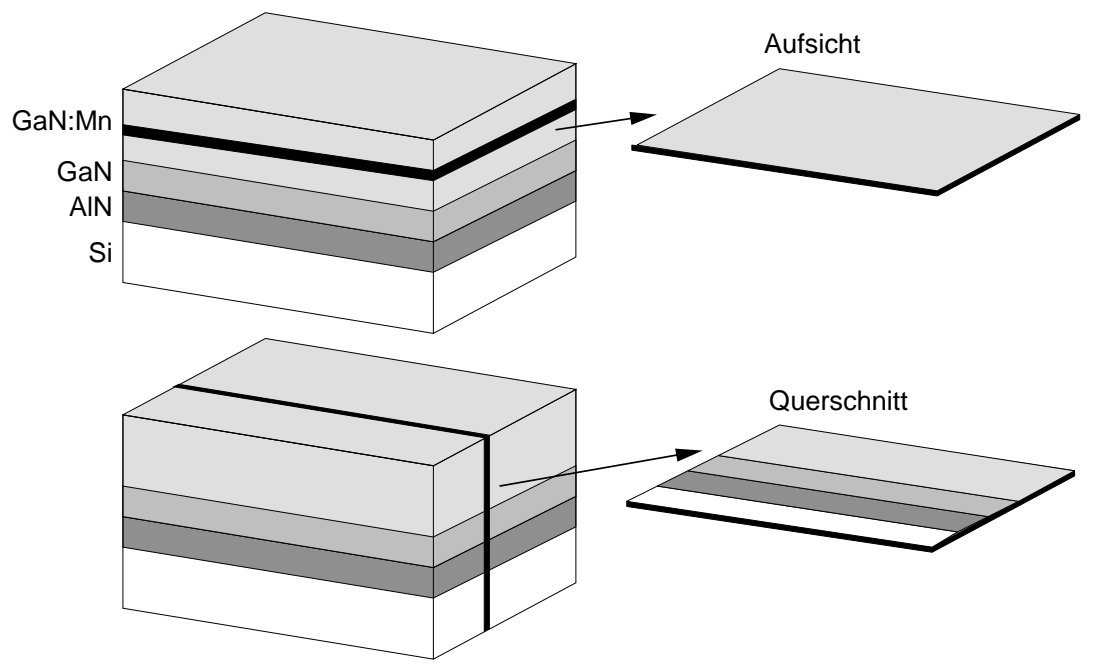

Abbildung 5.3: Schematische Probengeometrien. Die Schichtdicken sind übertrieben dargestellt.

\subsection{Basale Stapelfehler in GaN:Mn}

Wie in Abschnitt 6.2 noch ausführlicher dargestellt wird, wächst das GaN:Mn in Probe J0141 säulenartig. In den Abbildungen 5.4 und 5.5 sind Defokusserienrekonstruktionen von den Rändern zweier GaN:Mn-Säulen aus dieser Probe zu sehen. Um die Stapelfolge in den Gitterabbildungen zu erkennen, muss die Probe sehr dünn sein $(<10 \mathrm{~nm})$. Aufgrund der geringen Ausmaße der einzelnen Säulen, ihrer geringen Dicke und ihrer hohen Defektdichte war es nicht möglich, sie exakt zu orientieren. Die Abweichung von der Zonenachsenlage lässt sich gut in den linken Teilen der Objektwellenrekonstruktion am Verschmieren des Kontrastes erkennen. Ebenso führt die Verkippung zu einer stärkeren Anregung des kinematisch verbotenen (0001) Reflexes, was daran zu erkennen ist, dass sich die Muster der beiden Wurtzit-Halbebenen unterscheiden. Im rechten Bereich der Phasenrekonstruktion sind die Proben allerdings hinreichend dünn, so dass das Gitter trotz Probenverkippung sichtbar wird.

Die Positionen der Stapelfehler sind in den Abbildungen 5.4 und 5.5 eingezeichnet. Der größte Teil der identifizierten Stapelfehler war vom Typ I ${ }_{1}$. Die Stapelfehler durchlaufen die gesamte Säule, wie in den Hellfeldabbildungen zu erkennen ist. Es wurden keine Stapelfehler vom Typ E gefunden.

\subsection{Aufgespaltene $60^{\circ}$-Versetzung}

In Abb. 5.6a ist die Phasenrekonstruktion von zwei aufgespaltenen $60^{\circ}$-Versetzungen zu sehen (Probe J0141). Dieser Defekt befand sich in etwa $4 \mathrm{~nm}$ Abstand zur Grenzfläche zum GaNTemplate, die sich unterhalb des gezeigten Ausschnittes befand. Mittels der GPA konnten 

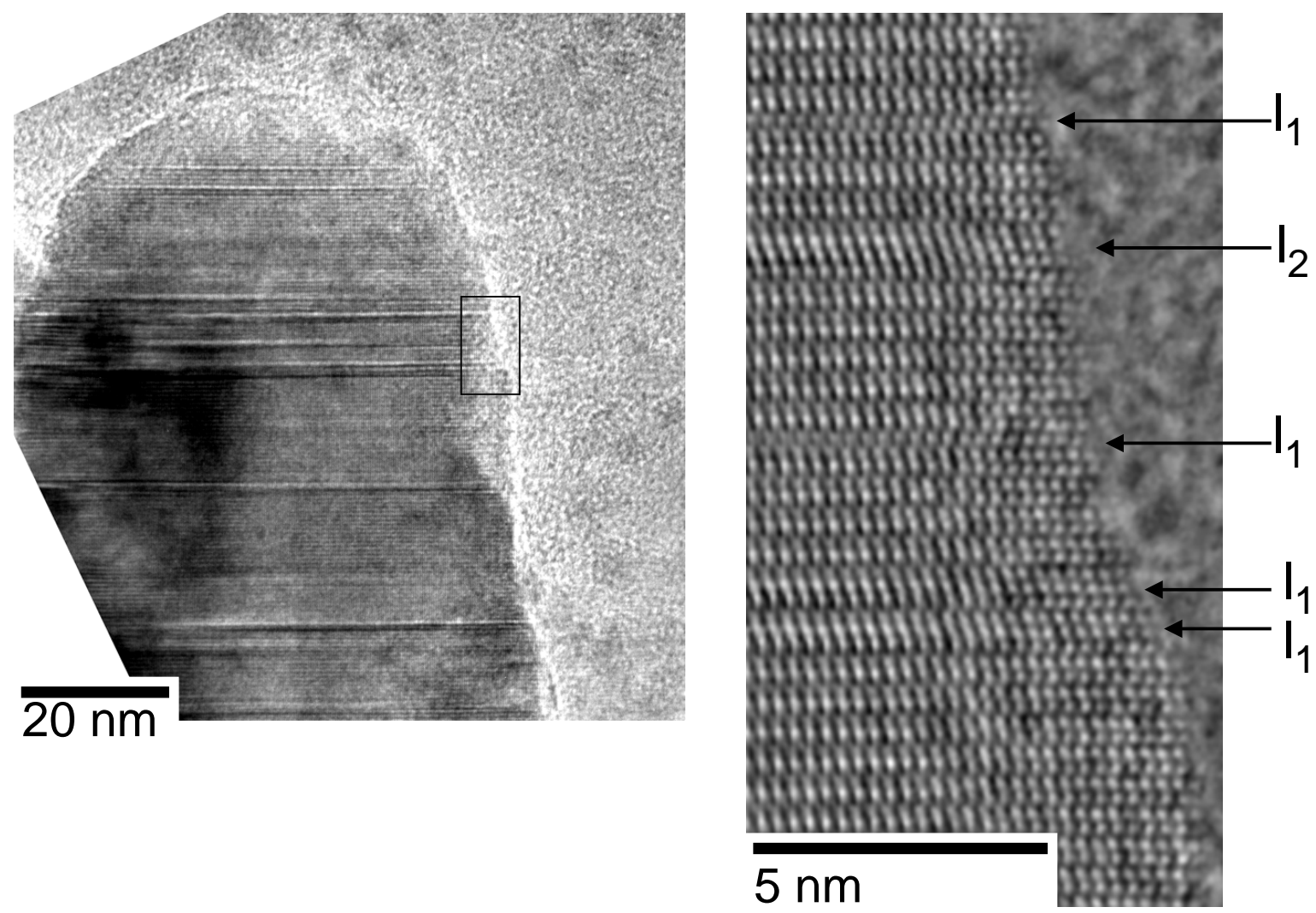

Abbildung 5.4: Links: Hellfeld-Aufnahme einer untersuchten Säule. Rechts: Phase der Objektwellen des angezeigten Ausschnittes. Die Maxima in der Phase entsprechen den Positionen der Ga-Atome, so dass hier die Fehler in der Stapelfolge leicht zu erkennen sind. 

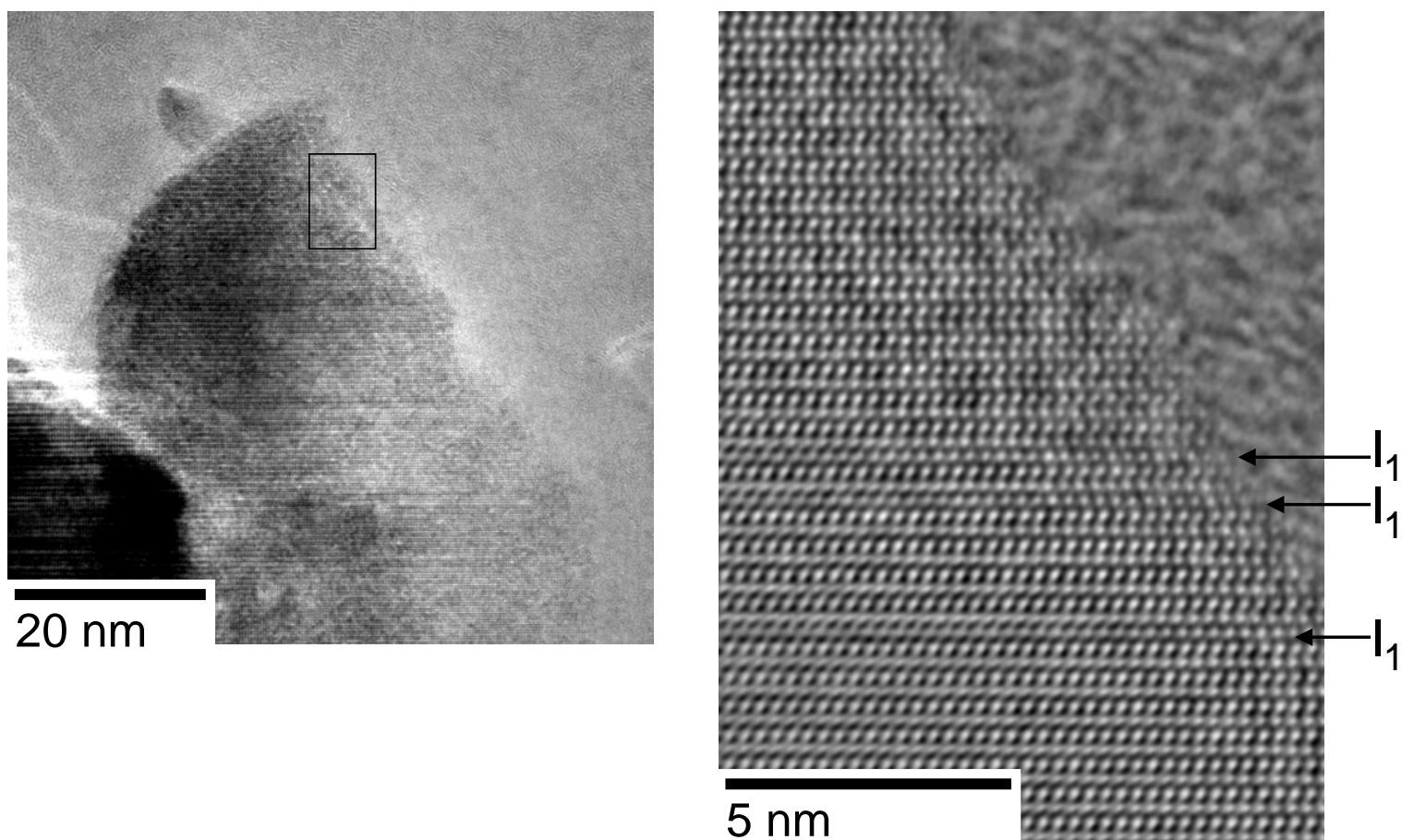

Abbildung 5.5: Links: Hellfeld-Aufnahme der untersuchten Säule. Rechts: Phase der Objektwellen des angezeigten Ausschnittes.

auch noch die Verschiebungsfelder, die Verzerrungsfelder und die lokale Rotation des Gitters gemessen werden. Die $x$-Komponente des Verschiebungsfeldes, $u_{x}$, ist in Abb. 5.6b gezeigt. Der Sprung zwischen maximalem und minimalen Wert kommt durch den Charakter der GPA zustande, die nur Verschiebungen bis auf Vielfache des entsprechenden Ebenenabstandes (hier $\left.\frac{1}{2}[1 \overline{1} 00]\right)$ messen kann, so dass dies in Wirklichkeit ein stetiger Übergang ist. Die projizierten Komponenten $\epsilon_{x x}$ und $\epsilon_{x y}$ des Verzerrungstensors sind für jeden Bildpunkt in Abbildungsteil c) und d) gezeigt, die Rotationskomponente $\omega_{x y}$ in Teil e). In $y$-Richtung (d.h. in [0001]-Richtung) waren keine signifikanten Komponenten der Verschiebung bzw. Verzerrung zu messen.

Für eine $60^{\circ}$-Versetzung mit dem Linienvektor [1120] ist die Aufspaltung in die beiden ShockleyPartialversetzungen gegeben durch die Burgersvektoren [17]:

$$
\frac{1}{3}[\overline{1} 2 \overline{1} 0] \rightarrow \frac{1}{3}[01 \overline{1} 0]+\frac{1}{3}[\overline{1} 100]
$$

wobei der Burgersvektor der linken Partialversetzung im $30^{\circ}$-Winkel und der der rechten im $90^{\circ}$-Winkel zum Linienvektor steht. Zwischen den Partialversetzungen entsteht ein $I_{2^{-}}$ Stapelfehler. Da die Abbildung entlang des Linienvektors ${ }^{1}$ aufgenommen wurde, ist nur die Projektion der Burgersvektoren auf die (11 $\overline{2} 0)$-Ebene zu erkennen:

$$
\frac{1}{2}[1100] \rightarrow \frac{1}{6}[1100]+\frac{1}{3}[\overline{1} 100] .
$$

\footnotetext{
${ }^{1}$ Die Wahl, ob der Linienvektor in das Papier hinein- oder aus dem Papier herauszeigt ist beliebig. In diesem Kapitel wird immer angenommen, dass der Linienvektor in das Papier hineinzeigt. Die in diesem Kapitel diskutierten Versetzungsreaktionen ließen sich analog für die andere Richtung aufschreiben.
} 
Der Burgersvektor der Schraubenkomponente könnte somit in das Papier hinein oder ihm heraus zeigen.

(a)
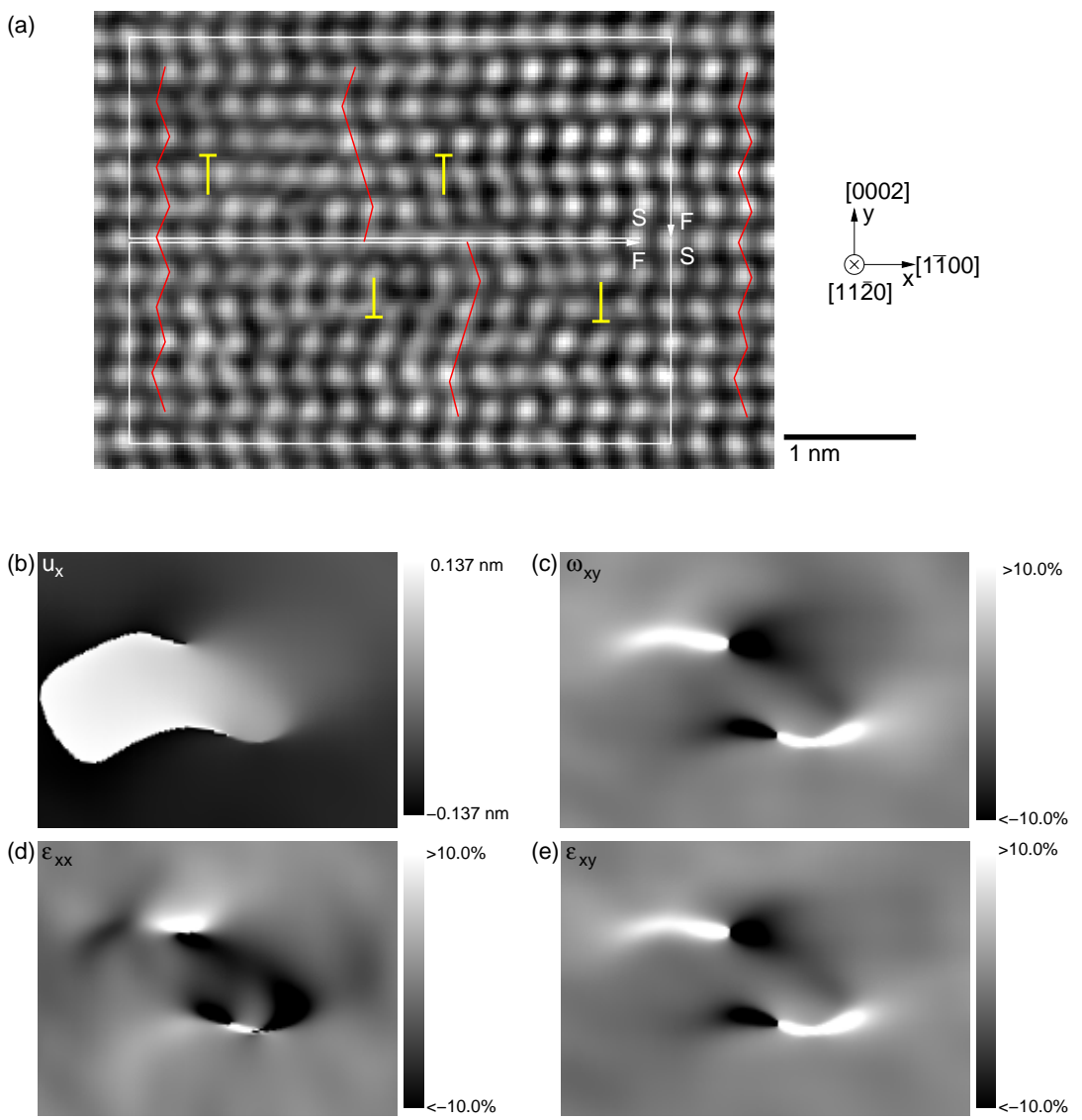

\begin{abstract}
Abbildung 5.6: Zwei entgegengesetzte aufgespaltene $60^{\circ}$-Versetzungen. Die Versetzungen sind aufgespalten in $30^{\circ}$-Partialversetzungen (beide links) und $90^{\circ}$-Partialversetzungen (rechts), zwischen denen sich jeweils ein basaler $\mathrm{I}_{2}$-Stapelfehler befindet. (a) Phasenrekonstruktion. (b) Verschiebungsfeld in [11̄00]-Richtung aus der GPA. (c)-(e) Verzerrungsfelder $\epsilon_{x x}$ und $\epsilon_{x y}$, sowie Rotation $\omega_{x y}$. Die GPA-Messungen zeigen den gleichen Ausschnitt wie die Phasenrekonstruktion.
\end{abstract}

Eine Analyse der unteren aufgespaltenen Versetzung in Abb. 5.6a durch einen Burgersumlauf ergibt, dass die Projektion des Burgersvektors $\boldsymbol{b}=\frac{1}{2}[1100]$ beträgt. Dabei wurde die SF $/ \mathrm{RH}-$ Konvention [17] benutzt, d.h. der Burgersvektor zeigt vom Start S zum Ende F („Finish“) des Umlaufs, der nach rechts gewunden (,right-handed“) ist. Der Burgersvektor des oberen Umlaufs ist entgegengesetzt, so dass sich die beiden Versetzungen insgesamt auslöschen, wie auch ein Burgersumlauf um beide Versetzungen ergibt. Aufgrund der Projektion kann nicht ermittelt werden, ob sich auch die Schraubenkomponenten der beiden $30^{\circ}$-Versetzungen aufheben. Diese könnten auch bei beiden Partialversetzungen in die gleiche Richtung zeigen, so dass beide Versetzungen zusammen eine $a$-Schraubenversetzung bilden würden.

In Abb. 5.7 ist ein Modell einer aufgespaltenen $60^{\circ}$-Versetzung gezeigt, deren Kerne Ga terminiert sind $(\beta$-Kern). Dabei wurde der Kern der Versetzungen ins Glide-Set der Struktur gelegt. Dieses Modell ist analog zu dem Modell aufgespaltener Versetzungen im Diamant-Gitter [17]. Es werden hier keine weiteren Annahmen über die Rekonstruktion der offenen Bindungen in 
den beiden Kernen gemacht. Für kubisches GaN [114] und für die $90^{\circ}$-Partialversetzung im hexagonalen GaN [115] wurden Berechnungen mit einer Kernstruktur wie die in diesem Modell durchgeführt. Die dabei vorhergesagten Rekonstruktionen ändern die Positionen der Atome im Kern der Versetzung nur leicht, was bei den untersuchten Dicken keinen großen Einfluss auf die resultierenden Objektwellenfunktionen hat.

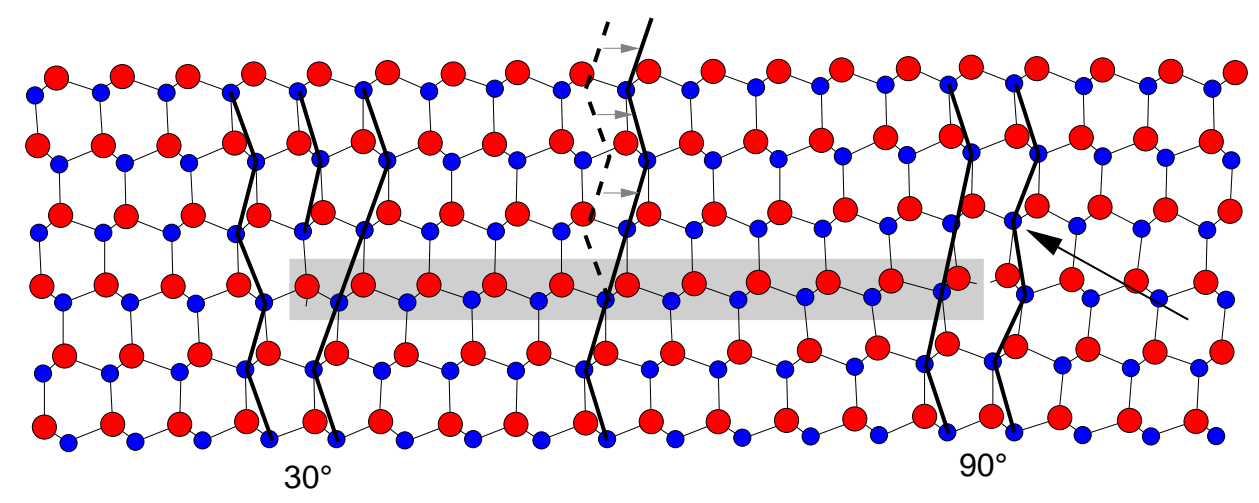

\begin{abstract}
Abbildung 5.7: Modell für eine aufgespaltene $60^{\circ}$-Versetzung. Die in Phasenrekonstruktionen sichtbaren Ebenen bestehen aus den N-Atomen (blau). Bei der $30^{\circ}$-Versetzung scheint eine Halbebene eingeschoben, während bei der $90^{\circ}$-Versetzung das Gitter verändert wirkt. Von unten über den Stapelfehler hinweg gesehen, wirkt das Gitter nach rechts abgeschert (s. Pfeile in der Mitte).
\end{abstract}

Ein Vergleich mit Simulationen ergab, dass die Probe in Abb. 5.6a eine Dicke von etwa $9.5 \mathrm{~nm}$ hat. Bei dieser Dicke entsprechen die Maxima der Phase den Positionen der N-Atomsäulen. Im Modell in Abb. 5.7 lässt sich erkennen, dass bei der $30^{\circ}$-Versetzung (links) eine zusätzliche (1100)-Ebene von oben eingeschoben scheint, während bei der $90^{\circ}$-Versetzung eine Veränderung des Gitters zu beobachten ist. Dies ermöglicht es, die beiden Partialversetzungen in der experimentellen Abbildung zu unterscheiden. Über den Stapelfehler hinweg gesehen (von unten nach oben) scheint das Gitter nach rechts abgeschert. Bei einer aufgespaltenen Versetzung mit umgekehrten Burgersvektor würde entsprechend die zusätzliche (1100)-Ebene von unten eingeschoben und die Abscherung des Gitters würde (wieder von unten nach oben gesehen) nach links erfolgen.

In Abb. 5.8a ist die obere der beiden aufgespaltenen Versetzungen zu sehen. Hier scheint bei der linken Partialversetzung eine Ebene von unten eingeschoben zu sein, so dass die linke Partialversetzung als $30^{\circ}$ - und die rechte als $90^{\circ}$-Versetzung identifiziert wird. Im Fall der unteren Versetzung, s. Abb. 5.9, scheint links eine Ebene von oben eingeschoben zu sein, so dass sich hier auch auf der linken Seite die $30^{\circ}$-Versetzung befindet. Aus den Wachstumsbedingungen der Probe ist bekannt, dass die Ga-Atome die obere Seite (in [0001]-Richtung) der Basalebenen besetzen. Aus der Abscherung des Gitters über die Stapelfehler hinweg und der Tatsache, dass sich die $30^{\circ}$-Versetzungen auf der linken Seite befinden, lässt sich schließen, dass die oberen Partialversetzungen N-terminiert ( $\alpha$-Kern) sind, während die unteren Ga-terminiert ( $\beta$-Kern) sind.

Um die beobachtete Objektwellenfunktion mit dem Versetzungmodell in Abb. 5.7 zu vergleichen wurde eine Simulation des gesamten Defektes, d.h. mit beiden Versetzungen, angefertigt. Dazu wurden die Verschiebungsfelder der vier Partialversetzungen, wie sie sich aus der anisotropen Elastizitätstheorie (s. Abschnitt B.1) ergeben, auf ein perfektes GaN-Gitter an- 
(a) Experiment

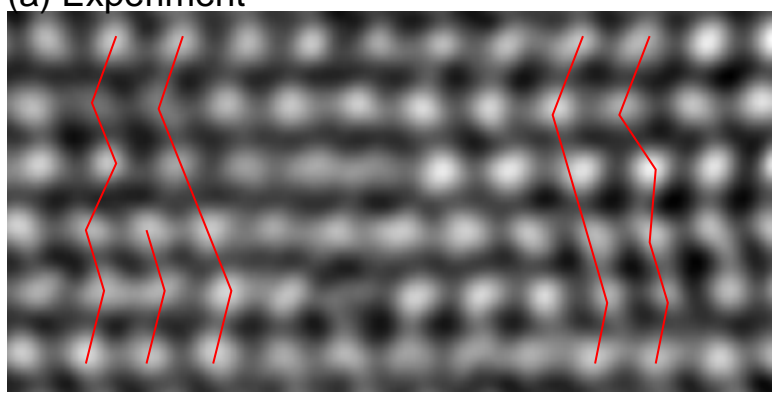

(b) Simulation

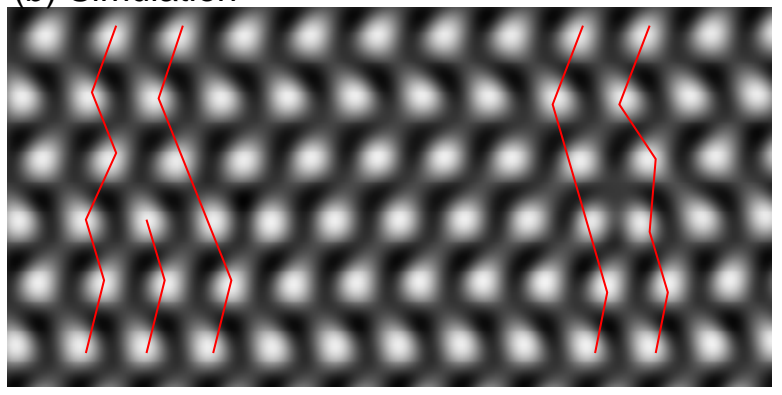

Abbildung 5.8: (a) Die obere aufgespaltene Versetzung aus Abb. 5.6. In der linken Partialversetzung scheint eine zusätzliche (1̄̄00)-Ebene eingeschoben. (b) Phase einer simulierten Objektwellenfunktion der Versetzung.
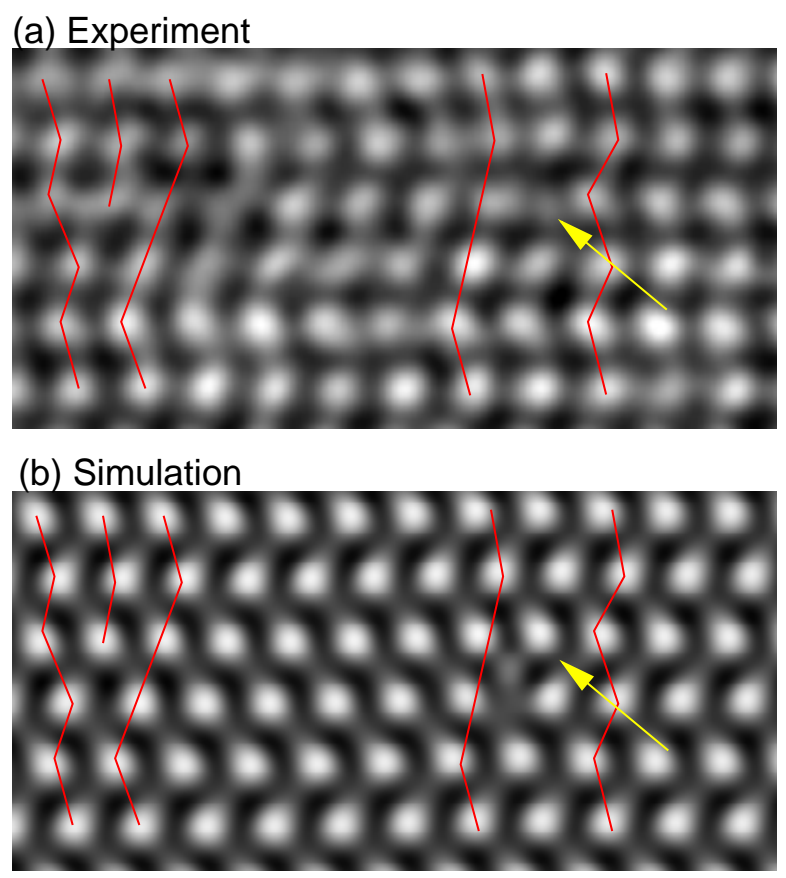

Abbildung 5.9: (a) Die untere aufgespaltene Versetzung aus Abb. 5.6. In der rechten Partialversetzung scheint eine zusätzliche (11̄00)-Ebene eingeschoben. (b) Phase einer simulierten Objektwellenfunktion der Versetzung. Die Simulation weicht in der mit einem Pfeil markierten Atomsäule sowie in der darunterliegenden von der Beobachtung ab. 
gewendet. Die Orte der Kerne der Versetzungen wurden dabei so in das Gitter gelegt, dass sich die Kernstrukturen wie in dem Modell bzw. die analogen $\alpha$-Kernstrukturen ergeben. Dabei soll die verwendetete Superposition der Verschiebungsfelder nur als Näherung dienen, das Gesamtverschiebungsfeld lässt sich sicherlich nur durch atomistische Simulationen berechnen. Allerdings sind nur kleine Abweichungen in den Atompositionen gegenüber dem verwendeten Modell zu erwarten. Die Schraubenanteile der beiden $30^{\circ}$-Versetzungen lagen in dieser simulierten Struktur entgegengesetzt, d.h. auch die beiden Versetzungen sind genau entgegengesetzt. Die Projektion des Verschiebungsfeldes für nicht entgegengesetzte Schraubenanteile sollte nur minimal vom verwendeten Fall abweichen.

Die Ergebnisse dieser Simulation sind in Abb. 5.8b und 5.9b gezeigt. Aufgrund der nicht in der Simulation berücksichtigten starken Verbiegungen in der Probe, die durch die naheliegende Grenzfläche zum Template und andere Defekte in der Nähe verursacht wurden, gibt die Simulation das beobachtete Muster nicht vollständig wider. Die Lagen der Maxima der Phasen, d.h. die Lagen der N-Atomsäulen, lassen sich allerdings trotzdem sowohl in der Simulation als auch im Experiment hinreichend gut ausmachen und miteinander vergleichen.

Im Vergleich stimmen die simulierte und beobachtete Phase in der oberen Versetzung (Abb. 5.8), sowie in der $30^{\circ}$-Partialversetzung der unteren Versetzung (Abb. 5.9 links) miteinander überein. In der unteren $90^{\circ}$-Versetzung (Abb. 5.9 rechts) jedoch ergibt sich eine Abweichung: Die mit dem Pfeil markierte Atomsäule scheint in der experimentellen Abbildung zu fehlen. Auch ist die Säule darunter im Experiment etwas weiter links zu finden. Beides zeigt, dass hier die zur Simulation benutzte Kernstruktur der $90^{\circ}$-Versetzung, wie sie auch von Savini et al. [115] angegeben wurde, nicht die beobachtete Struktur ist. Die oben beschriebenen Einschränkungen der Simulation können diese Abweichung alleine nicht erklären.

Die fehlende Atomsäule ist in Abb. 5.7 mit einem Pfeil markiert. Aufgrund der Abweichung der Muster dieser $90^{\circ}$-Versetzung zwischen Beobachtung und Simulation lässt sich ihre Lage nicht genau bestimmen. Die Versetzung könnte sich auch eine (1100)-Ebene weiter rechts befinden als hier angenommen. In diesem Fall ist es die Atomsäule in Abb. 5.7 links neben der markierten Säule, die ,fehlt“.

Hier bedarf es weiterer Modelle für die Kernstruktur dieser Partialversetzung. Da zur Aufstellung solcher Modelle atomistische Simulationen benötigt werden, liegt es außerhalb der Möglichkeiten dieser Arbeit, mögliche Kernstrukturen zu entwickeln. Auch in der Literatur gibt es keine ausführlichere Untersuchung über mögliche Kernstrukturen von Partialversetzungen am Rand von $I_{2}$-Stapelfehlern, wie sie beispielsweise für den $I_{1}$-Stapelfehler von Kioseoglou et al. [116] durchgeführt wurde.

Eine Beobachtung, wie diese Versetzungsreaktion, wäre ohne die Hilfe der Objektwellenrekonstruktion so nicht möglich gewesen. Durch die Delokalisierung wäre es nicht so direkt möglich gewesen, die genauen Orte der beiden Kern auszumachen. Auch ein Vergleich der Kernstrukturen hätte sich viel schwieriger gestaltet, da Bildsimulationen nötig geworden wären.

\section{Stapelfehlerenergie}

Aus der Breite des Stapelfehlers lässt sich mit Hilfe von anisotroper Elastizitätstheorie aus den Elastizitätskonstanten des Materials die Energie des Stapelfehlers bestimmen (s. Anhang B). Bei dieser Bestimmung wird davon ausgegangen, dass es sich um eine isolierte aufgespaltene 
Versetzung handelt und dass diese sich im thermodynamischen Gleichgewicht befindet. Ebenso wird davon ausgegangen, dass es keine weiteren Spannungen im Material gibt, die auf die Versetzung wirken.

Zwei parallele gleichsinnige Versetzungen, wie die beiden Partialversetzungen stoßen sich ab, so dass ihre Wechselwirkungsenergie kleiner wird, je weiter sie voneinander entfernt sind. Für den Stapelfehler zwischen den Partialversetzungen ist ein Energieaufwand proportional zu seiner Breite nötig. Im Gleichgewicht ist die Summe dieser entgegengesetzten Energiebeiträge für eine bestimmte Stapelfehlerbreite minimal.

Bei der Bestimmung der Breite des Stapelfehlers wäre die optische Delokalisierung, wie sie in der konventionellen HRTEM auftritt ein großes Problem, da sie die genaue Positionsbestimmung der Partialversetzungen verhindert, und es daher unmöglich macht die Breite des Stapelfehlers zu bestimmen. Mit der Objektwellenrekonstruktion hingegen ist es möglich, die Position auf eine projizierte Gitterebene genau zu bestimmen.

Suzuki et al. [20] haben in Weak-Beam-Aufnahmen die Breite einer in der Basalebenen gleitenden Versetzung für verschiedene Winkel zwischen Burgersvektor und Versetzungslinie bestimmt. Für $60^{\circ}$-Versetzungen erhalten sie eine Breite von rund $5.5 \mathrm{~nm}$, die auch in von ihnen gezeigten HRTEM-Abbildungen wiederzufinden ist. Um die Stapelfehlerenergie zu berechnen gehen sie den oben skizzierten Weg (s. Tabelle unten). Dabei benutzen sie die elastischen Konstanten, die von Savastenko und Sheleg [117] aus der temperaturabhängigen Verbreiterung von Röntgenbeugungs-Peaks abgeschätzt wurden, und erhalten eine Stapelfehlerenergie von $\gamma=1.37(25) \mathrm{meV} / \AA^{2}$. Wie Wright [118] diskutiert, ist die Genauigkeit dieser Konstanten fragwürdig, da die Werte sich merklich von neueren mit verschiedenen Verfahren gemessenen [119, 120, 121, 122] sowie berechneten Konstanten [118] unterscheiden.

Ein anderes experimentelles Ergebnis stammt von Zakharov et al. [21], die als typische Stapelfehlerbreite in HRTEM-Abbildungen $5.5 \mathrm{~nm}$ messen, was der Messung von Suzuki et al. entspricht. Sie berechnen eine Stapelfehlerenergie von $\gamma=2.49(25) \mathrm{meV} / \AA^{2}$, benutzen allerdings isotrope Elastizitätstheorie und machen die unrealistische Annahme, dass das PoissonVerhältnis verschwindet.

Die Breite der beiden Stapelfehler in Abb. 5.6a beträgt $1.40 \mathrm{~nm}$. Damit ergeben sich nach Gl. (B.1) folgende Gleichgewichtsstapelfehlerenergien:

\begin{tabular}{lc} 
Elastische Konstanten & Stapelfehlerenergie $\left[\mathrm{meV} / \AA^{2}\right]$ \\
\hline \hline (Savastenko et al. $[117])$ & 7.5 \\
Schwarz et al. $[121]$ & 15.3 \\
Yamaguchi et al.[119] & 18.0 \\
Takagi et al.[120] & 18.0 \\
Polian et al. $[122]$ & 18.6 \\
Wright[118] (theor.) & 17.4 \\
\hline
\end{tabular}

Die große Abweichung der hier berechneten Werte von den Literaturwerten, lässt sich bereits beim Vergleich der Breiten der Stapelfehlers sehen: hier beträgt sie rund ein Viertel der Breite von $5.5 \mathrm{~nm}$, die sowohl Suzuki et al. als auch Zakharov et al. bestimmt haben. Daher ist die im vorliegenden Fall berechnete Stapelfehlerenergie aus verschiedenen Gründen zweifelhaft: Die Formeln, die zur Berechnung der elastischen Energie benutzt wurden, berücksichtigen 
nicht die andere aufgespaltene Versetzung. Deren Partialversetzungen sind sich teilweise näher als die andere stapelfehlerberandende Partialversetzung. Daher ist zweifelhaft, ob sich dieses Versetzungspaar noch innerhalb der linearen Elastizitätstheorie behandeln lässt.

Wenn die Gesamtburgersvektoren der beiden aufgespaltenen Versetzungen sich gegenseitig aufheben, sollten sich auch im Gleichgewicht beide Versetzungen annihiliert haben. Auch kann gezweifelt werden, ob diese Versetzungen frei von weiteren Kräften sind, da sie sich nahe zu einer defektreichen Grenzfläche befinden, die weitere Verspannungen in das Gitter einbringt.

\subsection{Kleinwinkelkorngrenzen}

Wie in Abschnitt 6.2 noch ausführlicher besprochen wird, ist die GaN-Schicht in Probe J0141 säulenartig gewachsen. Diese Säulen sind dabei teilweise miteinander verwachsen, wie gut in Aufsichtsaufnahmen zu sehen ist (s. auch Abb. 6.11). In Abb. 5.10 ist eine hochauflösende TEM-Aufnahme in Aufsichtsgeometrie (Zonenachse [0001]) von zwei verwachsenen Säulen zu sehen, in der rechten oberen Ecke ist ein Säulenzwischenraum zu erkennen.

Leider ließen sich keine erfolgreichen Objektwellenrekonstruktionen in dieser Geometrie durchführen, so dass die Interpretation der Abbildungen schwierig ist. Erschwerend kommen starke Probenverzerrungen über den Ausschnitt hinweg hinzu, so dass sich das Gittermuster von Ort zu Ort ändert. So lassen sich beispielsweise in Bereich A die Intensitätsmaxima auf den Atomsäulen, in Bereich B in den Zwischenräumen und in Bereich C sogar auf beiden Orten finden.

Mithilfe einer Braggfilterung konnten trotzdem zwei Stufenversetzungen vom $a$-Typ mit Linienelement in $c$-Richtung ausgemacht werden, die innerhalb der stark gestörten Korngrenze liegen. Aus ihnen lässt sich abschätzen, dass etwa alle $15 \mathrm{~nm}$ eine (1010) Ebene in einem $30^{\circ}$ Winkel zur Grenzfläche eingefügt wird, was einen Winkel von rund $16 \mathrm{mrad}\left(0.9^{\circ}\right)$ zwischen den beiden Körnern ergibt.

\subsection{Simulationen von prismatischen Stapelfehlern}

Um die beiden möglichen Typen von prismatischen Stapelfehlen (Drum und Blank-Typ) in den folgenden Abschnitten 5.7 und 5.8.2 in Querschnittsaufnahmen unterscheiden zu können, wurden Simulationen der Objektwellen angefertigt. Die Ebenen dieser Stapelfehler liegen dabei schräg in der Probe. Die Geometrie der simulierten Probe ist in Abb 5.11 angedeutet. Dabei beginnt der Stapelfehler immer am gleichen Ort auf der Vorderseite der Probe, während er auf der Rückseite je nach Dicke die Probe an unterschiedlichen Orten verlässt. Die Verschiebungsvektoren in den Simulationen sind $\boldsymbol{p}_{\text {Drum }}=\frac{1}{2}[01 \overline{1} 1]$ für den Drum-Typ und $\boldsymbol{p}_{\text {Blank }}=\frac{1}{6}[02 \overline{2} 3]$ für den Blank-Typ.

Die Ergebnisse der Simulation des Drum-Stapelfehlers sind in Abb. 5.12 gezeigt, die des BlankStapelfehlers in Abb. 5.13. Ihre Muster unterscheiden sich deutlich. Das Muster des DrumStapelfehlers ist für beide (0002)-Halbebenen gleich: Die GaN-Paare überlappen zu horizontalen Zick-Zack-Linien in der Phase und der Kontrast in der Amplitude nimmt ab. Im Vergleich dazu zeigen die beiden Halbebenen beim Blank-Modell ein unterschiedliches Muster. In jeder zweiten Halbebene verschmiert das Muster sowohl in der Phase als auch in der Amplitude, wie 


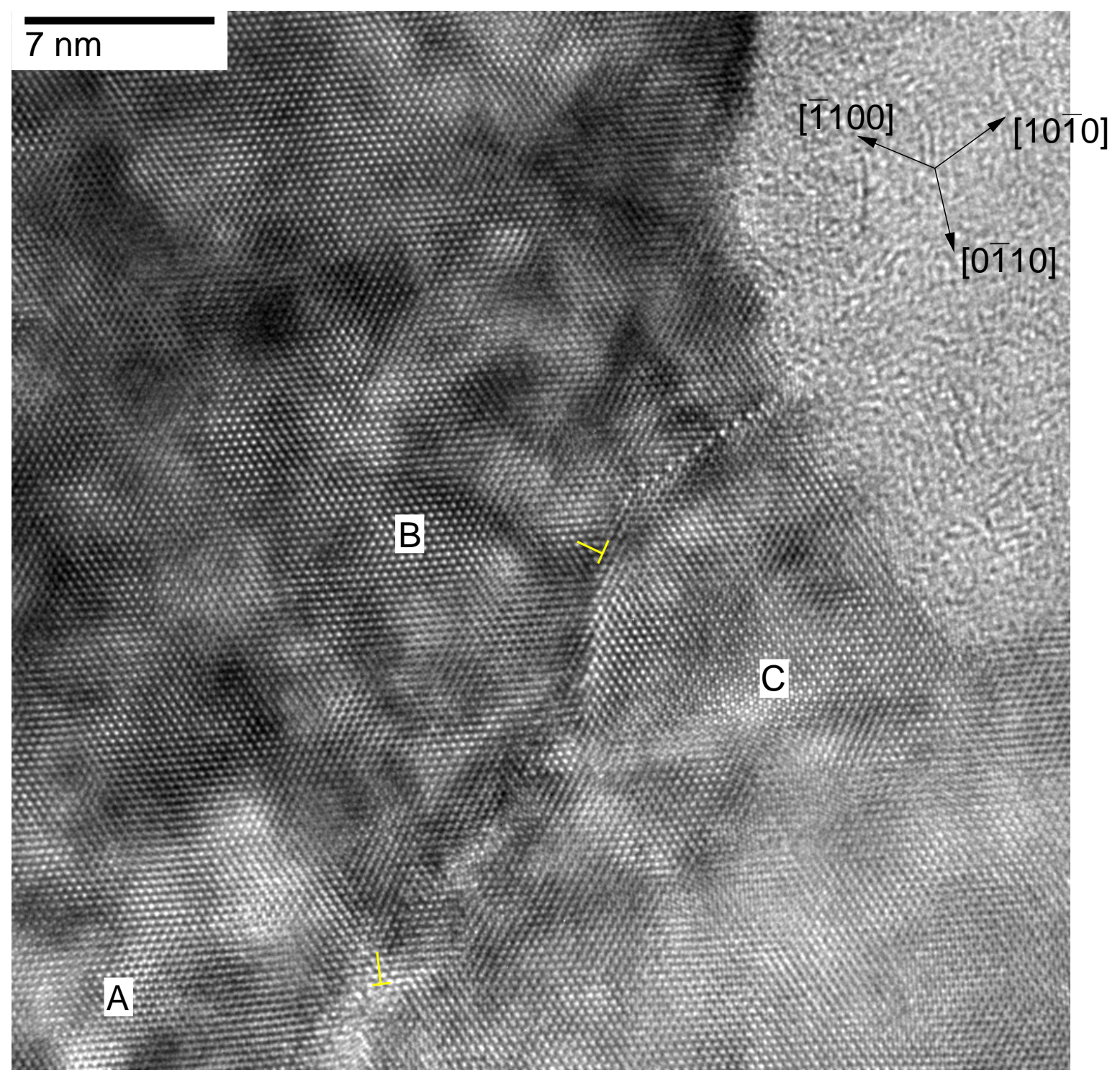

Abbildung 5.10: Hochaufösungsaufnahme einer Korngrenze. In dem gestörten Bereich sind zwei Versetzungen zu finden. In Bereich A liegen die Intensitätsmaxima auf den Atomsäulen, in Bereich B zwischen ihnen (also in den „Löchern“ der Struktur) und in Bereich C an beiden Orten.

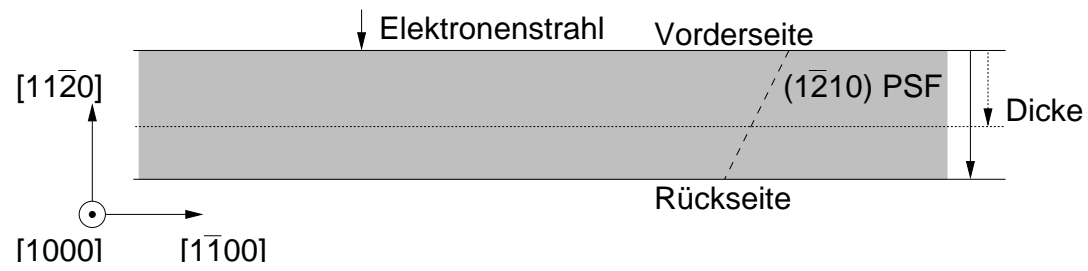

Abbildung 5.11: Probengeometrie der simulierten Stapelfehler. Der Stapelfehler beginnt immer am gleichen Ort auf der Vorderseite, während sein Ende von der Dicke der Probe abhängt. 
das auch beim Drum-Modell zu beobachten war. In den anderen Halbebenen hingegen scheint das Muster der Ga-Atome in den Phasen schärfer zu werden, während in der Amplitude keine große Änderung zum ungestörten GaN sichtbar wird. In den Phasen des Drum-Modells lässt sich zusätzlich ein 6-zähliges Muster finden, ein vergleichbares Muster findet sich beim Blank-Stapelfehler nicht.
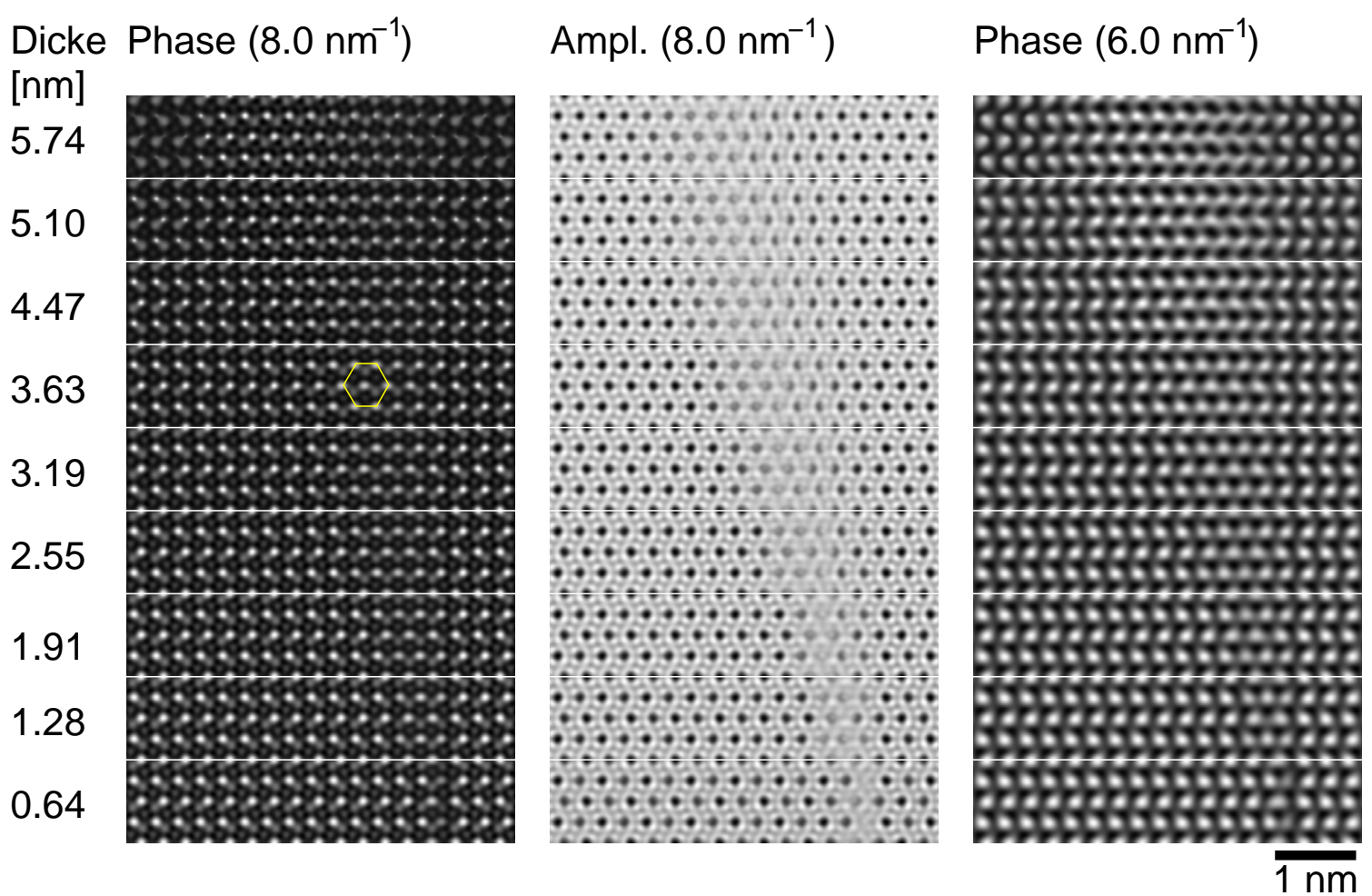

Abbildung 5.12: Simulation eines Drum-Stapelfehlers für verschiedene Dicken. Gezeigt sind die Phase und Amplitude der Objektwelle für Raumfrequenzen bis $8.0 \mathrm{~nm}^{-1}$ und die Phase der Welle für Raumfrequenzen bis $6.0 \mathrm{~nm}^{-1}$. Beide (0002)-Halbebenen zeigen ein gleiches Muster. Charakteristisch ist auch das typische sechszählige Muster im Bereich des Stapelfehlers, das in gelb angedeutet ist.

Eine weitere Möglichkeit der Unterscheidung bietet das Verschiebungsfeld des Stapelfehlers, das sich mit der GPA messen lässt. Entlang der $c$-Achse lässt sich dabei allerdings der (0001)Reflex schlecht nutzen, da er kinematisch verboten und deshalb nur sehr schwach in den Objektwellen angeregt ist. Daher wird die GPA am (0002)-Reflex durchgeführt, d.h. es lassen sich nur Verschiebungen bis auf ein Vielfaches von $\frac{1}{2}$ [0001] messen. Daher lassen sich allerdings nicht mehr die Verschiebungen der beiden Stapelfehler entlang der $c$-Achse messen, da sie beide $\frac{1}{2}[0001]$ betragen. Senkrecht dazu lässt sich die GPA am (1100)-Reflex durchführen, was es erlaubt, Verschiebungen bis auf Vielfache von $\frac{1}{2}[1 \overline{1} 00]$ zu messen.

Die GPA wurde an den simulierten Objektwellen für eine Dicke von $2.55 \mathrm{~nm}$ bei einer Apertur von $8.0 \mathrm{~nm}^{-1}$ und für eine Dicke von $5.77 \mathrm{~nm}$ bei einer Apertur von $6.0 \mathrm{~nm}^{-1}$ durchgeführt. Profile der Verschiebungen in [11̄00]-Richtung über den Stapelfehler hinweg (d.h. entlang der [11̄00]-Richtung) sind in Abb. 5.14 gezeigt. Für einen Stapelfehler vom Blank-Typ betragen die Verschiebungen etwa $\frac{1}{6}[1 \overline{1} 00]$, während sie für den Drum-Typ $\frac{1}{4}[1 \overline{1} 00]$ betragen. Dies sind auch die Verschiebungen, die aus der Projektion des Verschiebungsvektors auf die [11̄00]-Richtung 


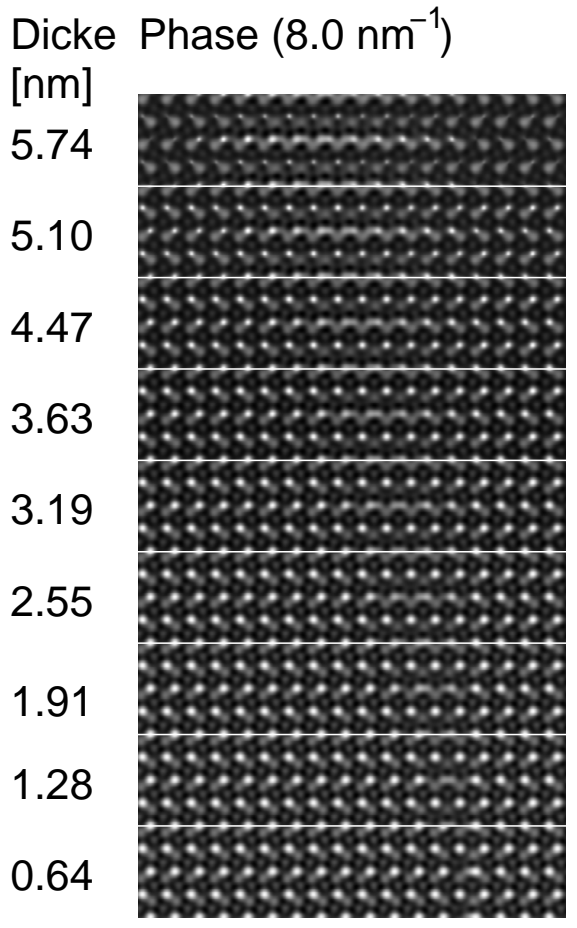

Ampl. $\left(8.0 \mathrm{~nm}^{-1}\right)$

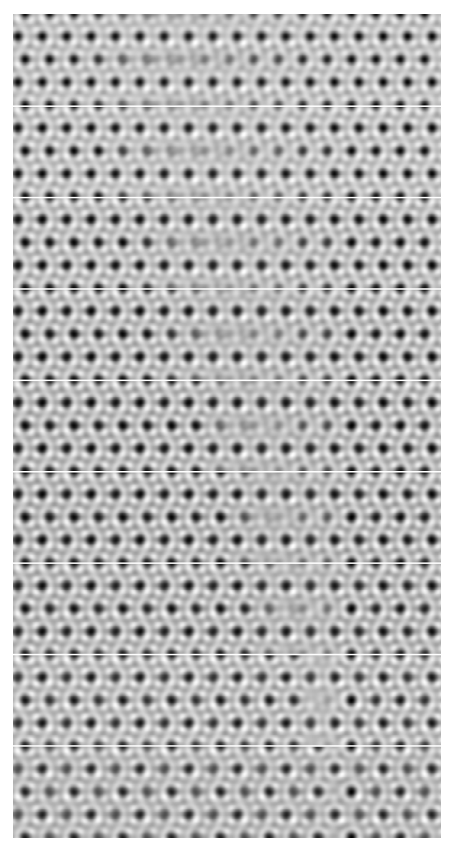

Phase $\left(6.0 \mathrm{~nm}^{-1}\right)$

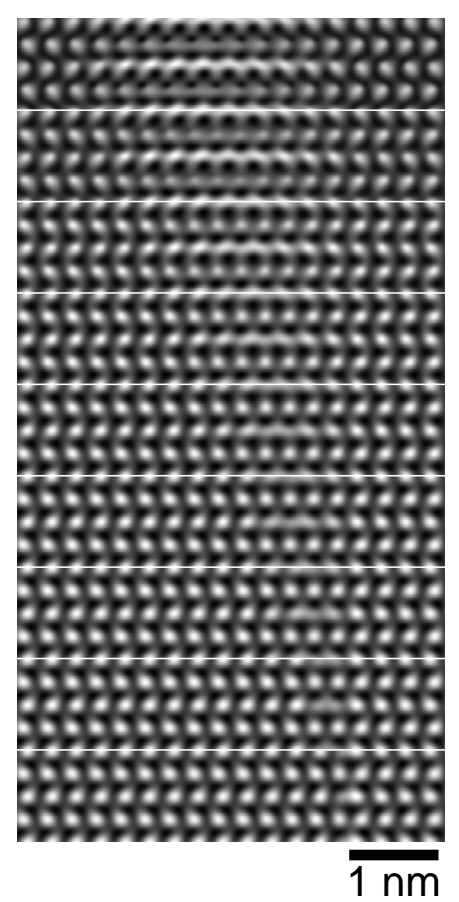

Abbildung 5.13: Simulation eines Blank-Stapelfehlers für verschiedene Dicken. Gezeigt sind die Phase und Amplitude der Objektwelle für Raumfrequenzen bis $8.0 \mathrm{~nm}^{-1}$ und die Phase der Welle für Raumfrequenzen bis $6.0 \mathrm{~nm}^{-1}$. Die Muster der beiden (0002)-Halbebenen unterscheiden sich deutlich: Während in der einen Halbebene die GaN-Paare überlappen und so die Horizontalen zu einer verschmierten Zick-Zack-Linie in der Phase werden bzw. der Kontrast in der Amplitude verschwindet, zeigen die anderen Halbebenen scharfe Punkte in der Phase bzw. keine sichtbaren Veränderungen in der Amplitude. 
zu erwarten sind. Diese Simulation zeigt, dass das mittels GPA gemessene Verschiebungsfeld auch genutzt werden kann, um den Stapelfehlertyp zu identifizieren, wenn die Defektebene schräg zum Elektronenstrahl in der Probe liegt.

(a) Blank-Typ

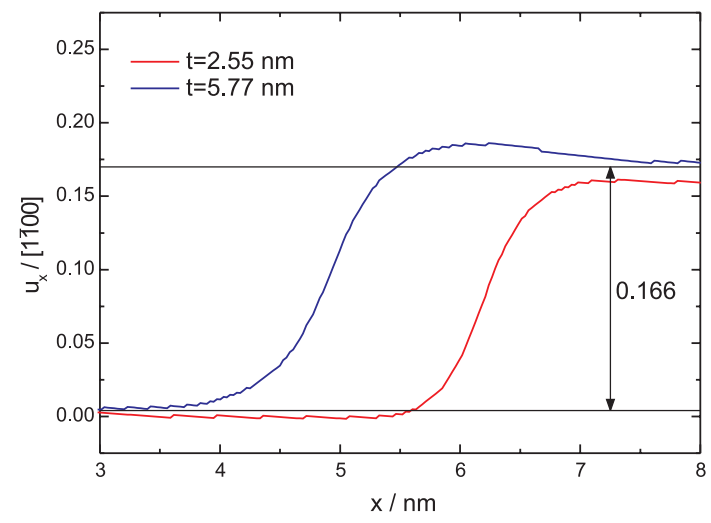

(b) Drum-Typ

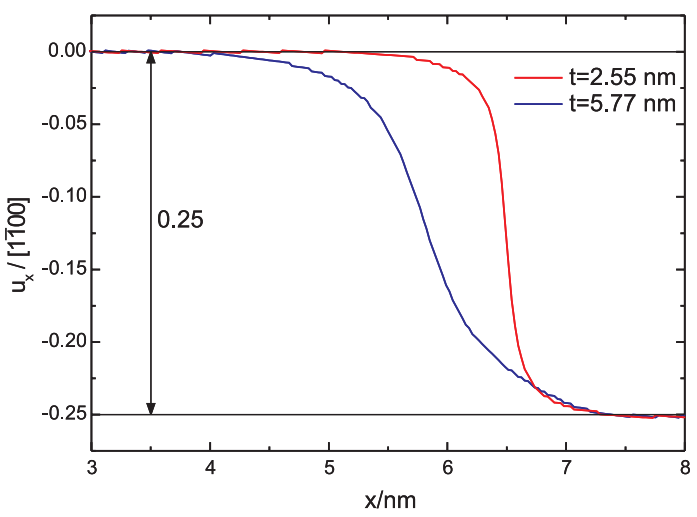

Abbildung 5.14: Mittels GPA in simulierten Objektwellen bestimmte Verschiebungsprofile für verschiedene Probendicken $t$ und Aperturen (s. Text). Die Profile laufen über den prismatische Stapelfehler hinweg. (a) Blank-Typ, die Verschiebung beträgt rund $\frac{1}{6}[1 \overline{1} 00]$. (b) Drum-Typ, hier beträgt die Verschiebung rund $\frac{1}{4}[1 \overline{1} 00]$.

\subsection{Aufgespaltene $c(+a)$-Versetzung}

In Abb. 5.15 ist die Objektwellenrekonstruktion eines prismatischen Stapelfehlers sowie zweier Partialversetzungen an seinem Rand in einer GaN Probe (J0145) zu erkennen. Der Typ des Stapelfehlers wird auf zwei unterschiedliche Weisen als Blank-PSF identifiziert.

1. Beim Vergleich des Musters des Stapelfehlers in der Objektwelle mit den Mustern, die nach den Simulationen in Abb. 5.12 und 5.13 zu erwarten sind, findet sich das Muster des Blank-Modells wieder: Die beiden (0002)-Halbebenen zeigen einen unterschiedlichen Kontrast. Der Kontrast der einen Halbebene verschmiert, sowohl in der Phase als auch in der Amplitude, während der Kontrast der anderen Halbebene keine große Änderung zeigt. Auch lässt sich aus dem Vergleich der Breiten des verschmierten Bereichs in Abbildung und Simulation schließen, dass die Probendicke hier $2.0 \mathrm{~nm}$ bis $2.5 \mathrm{~nm}$ beträgt.

2. Das Verschiebungsprofil aus der GPA zeigt eine Verschiebung von rund $\frac{1}{3}[1 \overline{1} 00]$ über den Stapelfehler hinweg (s. Abb. 5.16). Da die Verschiebung nur bis auf Vielfache von $\frac{1}{2}[1 \overline{1} 00]$ gemessen werden kann, entspricht dies der erwarteten Verschiebung des BlankTyps $\frac{1}{6}[1 \overline{1} 00]=\frac{1}{2}[1 \overline{1} 00]-\frac{1}{3}[1 \overline{1} 00]$. Für den Drum-Typ hingegen würde eine Verschiebung von $\frac{1}{4}[1 \overline{1} 00]$ erwartet werden.

Die Probe ist mit dieser Dicke hinreichend dünn, um die Atome im unverzerrten Bereich seitlich des Stapelfehlers direkt aufzulösen, wie in Abb. 5.17 gezeigt wird. Die Dicke des GaN befindet sich noch weit unterhalb der Extinktionslängen der bildbestimmenden Strahlen (der 
(a) Phase

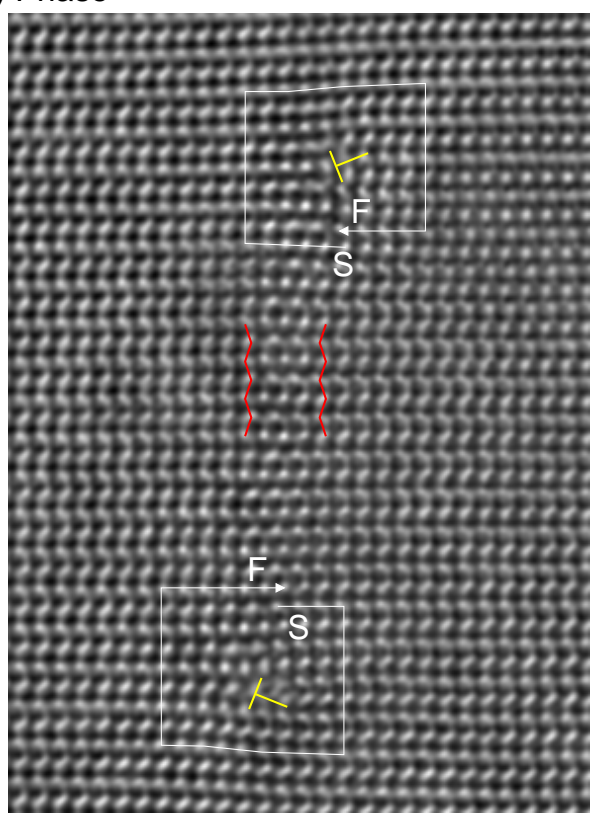

(b) Amplitude

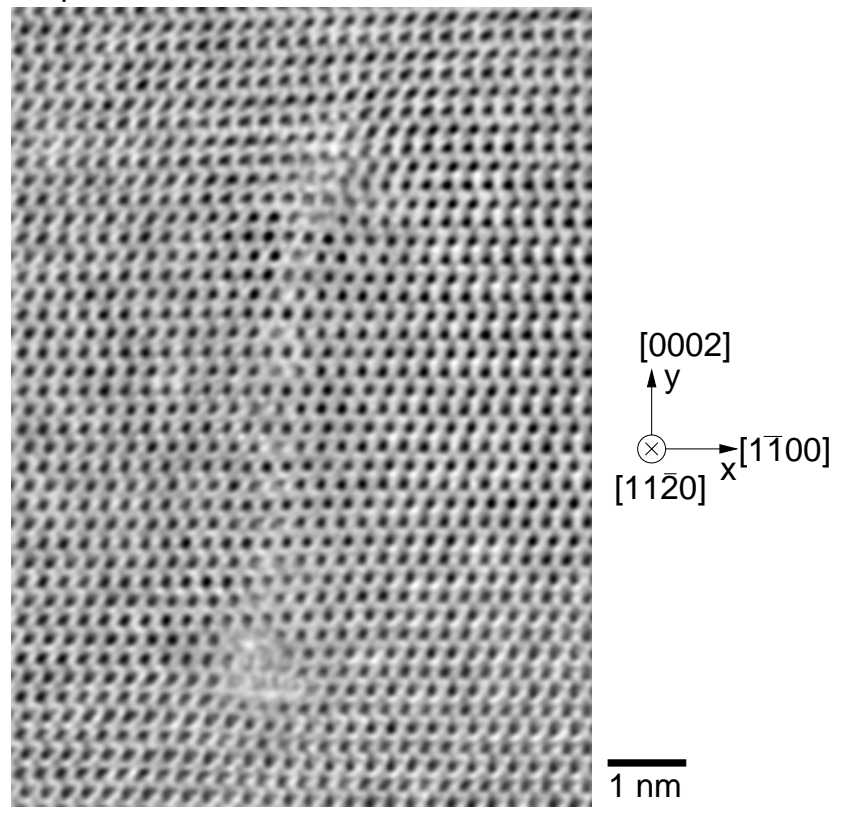

(c) $u_{x}$
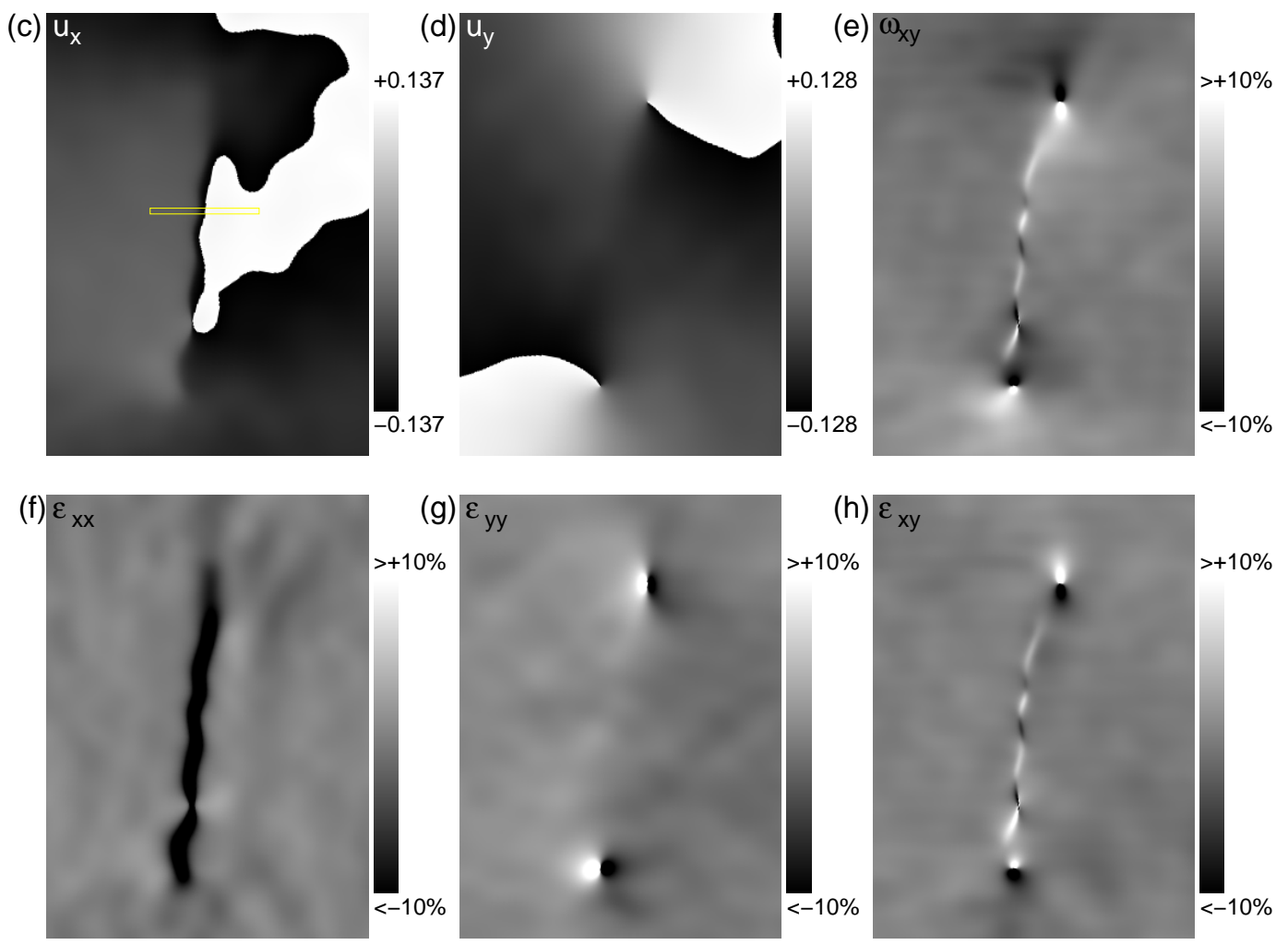

(g) $\varepsilon_{y y}$
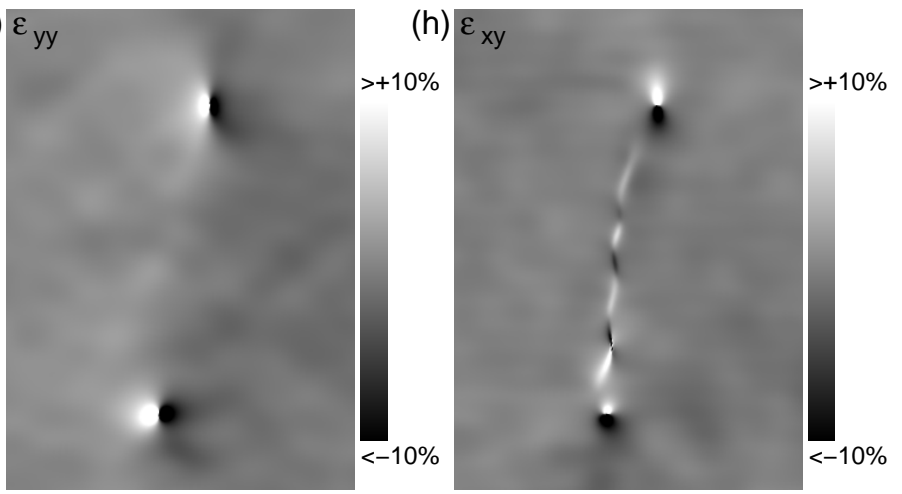

Abbildung 5.15: Prismatischer Stapelfehler berandet von zwei Partialversetzungen: (a) Phase der Defokusserienrekonstruktion zusammen mit angedeuteten Burgersumläufen. (b) Zugehörige Amplitude. (c) Verschiebungsfeld in [1100]-Richtung aus GPA (in nm). (d) Entsprechend in [0002]-Richtung (in nm). (e)-(h) Rotation $\omega_{x y}$ und Verzerrungsfelder $\epsilon_{x x}, \epsilon_{y y}$ sowie $\epsilon_{x y}$ aus der GPA. 


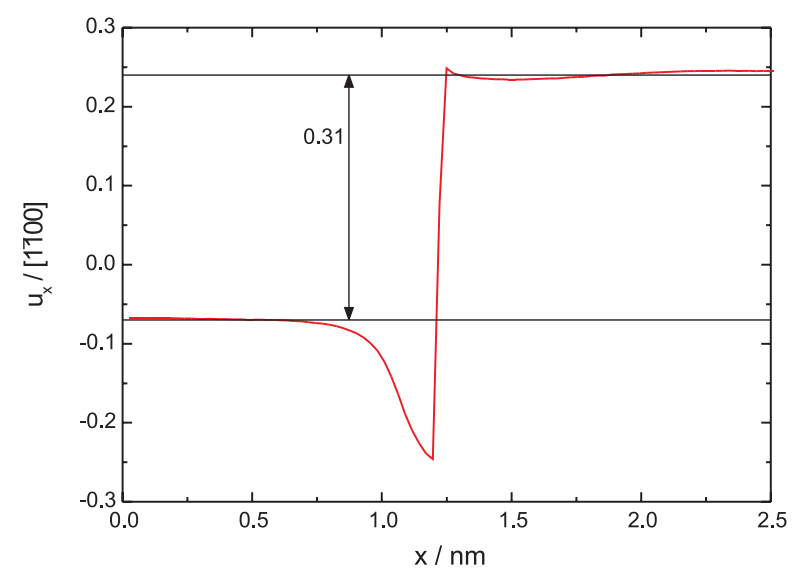

Abbildung 5.16: Verschiebungsprofil in [1]100]-Richtung aus dem markierten Bereich in Abb. 5.15c. Da der Abstand nur bis auf Vielfache des Ebenenabstands des benutzten Reflexes von $\frac{1}{2}[1 \overline{1} 00]$ gemessen werden kann, entspricht die gemessene Verschiebung von rund $\frac{1}{3}[1 \overline{1} 00]$ der Verschiebung des Blank-Stapelfehlers von $\frac{1}{6}[1 \overline{1} 00]=\frac{1}{2}[1 \overline{1} 00]-\frac{1}{3}[1 \overline{1} 00]$.

$(000 \overline{2})$-Strahl hat eine Extinktionslänge von rund $10 \mathrm{~nm})$, so dass eine solche direkte Interpretation möglich ist. Der Vergleich mit der Simulation zeigt auch die Gültigkeit dieser Deutung. Die Ga-Atome zeigen aufgrund ihrer höheren Ordnungszahl einen größeren Kontrast als die N-Atome. Der Abstand der GaN-Dumbbells befindet sich zwar mit $1.13 \AA$ unterhalb des Informationslimits des Mikroskops, aufgrund des unterschiedlich starken Kontrastes der beiden Atomsorten lässt sich aber noch die Orientierung und Polarität erkennen.

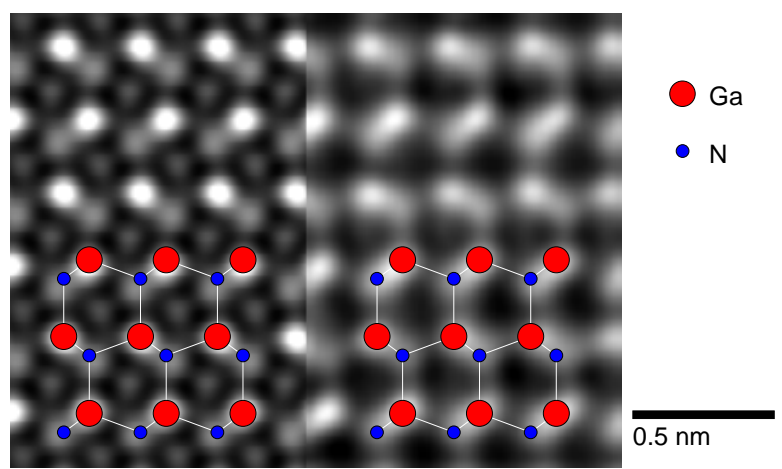

\footnotetext{
Abbildung 5.17: Rechts: Vergrößerung der Phasenrekonstruktion eines unverzerrten Bereiches. Die beiden Atomsorten und die Gitterstruktur lassen sich direkt auflösen. Links: Simulation für eine Probendicke von 2.55 $\mathrm{nm}$.

Um diesen Stapelfehler zu erzeugen, muss die Versetzungslinie in der Ebene des Stapelfehlers liegen. Es ergeben sich zwei verschiedene Stapelfehlerebenen, die in der Projektion nicht zu

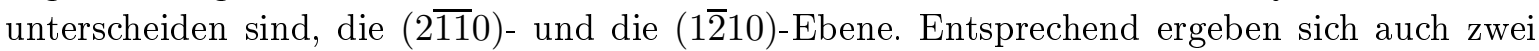
Richtungen für Versetzungslinien. Mit der Festlegung, dass die Linienelemente in das Papier

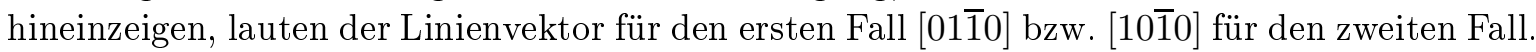
Im Folgenden wird nur der Fall einer Stapelfehlerebene von (1210) und einer Versetzungslinie entlang $[10 \overline{1} 0]$ besprochen, der andere Fall ergibt sich analog.
} 
Burgersumläufe nach SF/RH-Konvention ergeben für die untere Partialversetzung einen Burgersvektor von $\frac{1}{6}[1 \overline{1} 03]$, für die obere Partialversetzung $\frac{1}{6}[1103]$ und für einen Umlauf um den gesamten Stapelfehler [0001]. Damit ergibt sich als Versetzungsreaktion in der Projektion auf die (11̄̄0)-Ebene:

$$
\frac{1}{3}[0003] \rightarrow \frac{1}{6}[1 \overline{1} 03]+\frac{1}{6}[\overline{1} 103]
$$

Aus kristallographischen Überlegungen müssen die beiden Partialversetzungen vom FrankShockley-Typ $\frac{1}{6}\langle 20 \overline{2} 3\rangle$ sein, um die nötigen Verschiebungen des Stapelfehlers zu erzeugen. Da neben den besprochenen keine weiteren Kristalldefekte auszumachen sind, muss der Gesamtburgersvektor der einer perfekten Versetzung sein. Es ergeben sich zwei verschiedene Typen von Versetzungsreaktionen, die kompatibel zu der beobachteten Projektion sind.

Eine Möglichkeit ist die Reaktion

$$
[0001] \rightarrow \frac{1}{6}[20 \overline{2} 3]+\frac{1}{6}[\overline{2} 023]
$$

Die basalen Anteile der beiden Partialvektoren heben sich hier gerade auf, so dass diese Reaktion der Aufspaltung einer perfekten $c$-Sufenversetzung entspricht. Eine solche Versetzung

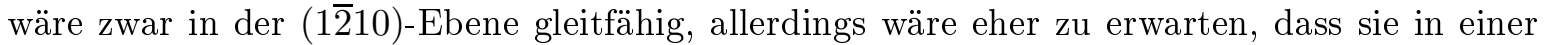
$\{1 \overline{1} 00\}$-Ebene gleitet. Der Ebenenabstand der $\{1 \overline{2} 10\}$-Ebenen beträgt $1.6 \AA$, der der $\{1 \overline{1} 00\}$ Ebenen aber $2.8 \AA$, was eine geringere Peierls-Energie vermuten lässt. Ebenso ist auch eher eine Aufspaltung dieser Versetzung unter Erzeugung eines basalen $\mathrm{I}_{1}$-Stapelfehlers als eines prismatischen Stapelfehlers zu erwarten [17]. Diese Aufspaltung mit einem I $\mathrm{I}_{1}$-BSF wurde auch bereits in GaN beobachtet [15].

Die andere mögliche Versetzungsreaktion lautet

$$
\frac{1}{3}[\overline{11} 23] \rightarrow \frac{1}{6}[0 \overline{2} 23]+\frac{1}{6}[\overline{2} 023] .
$$

Hier haben die beiden Partialversetzungen zusammen zusätzlich zum $c$-Anteil auch einen $a$ Anteil entlang der Elektronenstrahlrichtung, so dass diese Reaktion insgesamt der Aufspaltung einer $(a+c)$-Versetzung entspricht. Diese Versetzung wäre nicht gleitfähig, da der Burgersvektor nicht in einer Gleitebene liegt.

Da die Versetzungenslinien schräg in der Probe liegen, sind die beiden möglichen Versetzungsreaktionen in dieser Projektion nicht voneinander zu unterscheiden. Auch eine Unterscheidung mithilfe von Simulationen ist nicht möglich, da bis jetzt keine Kernstrukturen für diese Partialversetzungen in der Literatur vorgeschlagen wurden.

\section{Stapelfehlerenergie}

Wie schon bei den aufgespaltenen $60^{\circ}$-Versetzungen lässt sich auch hier die Stapelfehlerenergie aus dem Abstand der beiden Partialversetzungen mittels der Formeln der anisotropen Elastizitätstheorie (s. Anhang B) berechnen. Der hier vorliegende Stapelfehler hat eine Breite von $7.40 \mathrm{~nm}$. Es ergeben sich als Stapelfehlerenergien (in $\mathrm{meV} / \AA^{2}$ ) für die Annahme einer aufgespaltenen $c$ - bzw. $(a+c)$-Versetzung bei einem geschätzten relativen Fehler von 1\%: 


\begin{tabular}{lcc} 
Elastische Konstanten & $a$-Versetzung & $(a+c)$-Versetzung \\
\hline \hline (Savastenko et al..[117]) & $(2.9)$ & $(7.4)$ \\
Schwarz et al.[121] & 3.4 & 12.6 \\
Yamaguchi et al.[119] & 7.9 & 18.7 \\
Takagi et al.[120] & 7.7 & 18.5 \\
Polian et al. $[122]$ & 8.1 & 19.2 \\
Wright[118] (theor.) & 8.1 & 18.5 \\
\hline
\end{tabular}

Diese Werte sind rund eine Größenordnung kleinen als die erwarteten theoretischen Werte $\left(\approx 140 \mathrm{meV} / \AA^{2}[19]\right)$, d.h. auch der Abstand der Partialversetzungen ist entsprechend größer als zu erwarten wäre.

Diese Abweichung kann mehrere Gründe haben. Es lässt sich bereits in der Abbildung 5.15 beobachten, dass die Stapelfehlerebene klettert: die beiden Partialversetzungen müssten übereinander liegen, aber ihre Verbindungslinie ist um etwa $10^{\circ}$ gegen die $c$-Achse geneigt. Auch im Scherungs-Verzerrungsfeld $\epsilon_{x y}$ und im Rotationsfeld $\omega_{x y}$ lassen Verzerrungen zwischen den beiden Partialversetzungen erkennen, die für einen ,perfekten“ Stapelfehler nicht zu erwarten wären.

Es ist möglich, dass die Partialversetzungen nicht in den für das Gleichgewicht nötigen Abstand gleiten können, da im Bereich der Verzerrungen innerhalb des Stapelfehlers zum Gleiten sicherlich höhere Energiebarrieren zu überwinden sind, wofür die thermische Energie evtl. nicht ausreicht. Wenn es sich um eine $(a+c)$-Versetzung handelt, wäre eine der beiden Partialversetzungen auch nicht gleitfähig, so dass das Erreichen des thermodynamischen Gleichgewichts erschwert ist. Auch kann nicht garantiert werden, dass sich die Versetzungen frei von weiteren Kräften sind.

\subsection{Weitere Partialversetzungen}

\subsubsection{Spiegelvarianten}

In Abb. 5.18 sind zwei Spiegelvarianten des basalen $\mathrm{I}_{1}$-Stapelfehlers zu erkennen. Die beiden Varianten ergeben sich aus den beiden möglichen Ebenenfolgen dieses Stapelfehlers: zum einen die Stapelfolge ...ABABCBCBC..., zum anderen ...ABABACACA.... Der Burgersumlauf ergibt für die Partialversetzung in Abb. 5.18a in der Projektion einen Burgersvektor von $\boldsymbol{b}=\frac{1}{6}[1 \overline{1} 00]$ bei einem Linienelement in Richtung von [11 $\left.\overline{2} 0\right]$. Ebenso lässt sich das Klettern des Stapelfehlers um $c / 2$ erkennen. Aus kristallographischen Überlegungen sind sowohl das Klettern des Stapelfehlers als auch ein Burgersvektor der Art $\frac{1}{3}<10 \overline{1} 0>$ zu erwarten [123].

Da die Komponente des Burgersvektors entlang der Strahlrichtung nicht zu erkennen ist, kann die Schraubenkomponente des Burgersvektors wieder in das Papier hinein oder hinaus zeigen, so dass als Burgersvektoren der Partialversetzungen im Einklang mit obiger Projektion $\boldsymbol{b}=\frac{1}{3}[10 \overline{1} 0]$ und $\boldsymbol{b}=\frac{1}{3}[0 \overline{1} 10]$ in Frage kommen.

\subsubsection{Stairrod-Versetzung}

In Abb. 5.19 ist das Umklappen eines prismatischen Stapelfehlers in einen basalen Stapelfehler vom Typ $I_{1}$ zu sehen (Probe J0141). Der genaue Verlauf des Stapelfehlers ist im Bereich des 
(a)

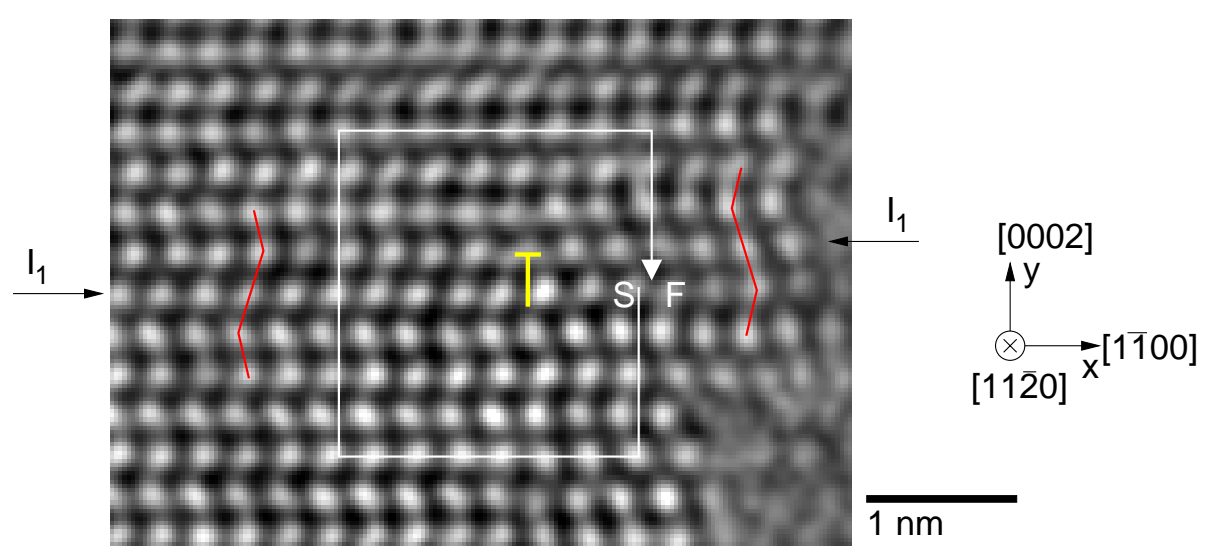

(b)

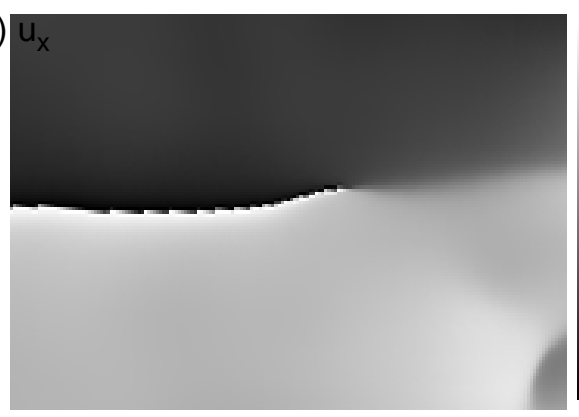

$0.137 \mathrm{~nm}$

(d) $\varepsilon_{x x}$

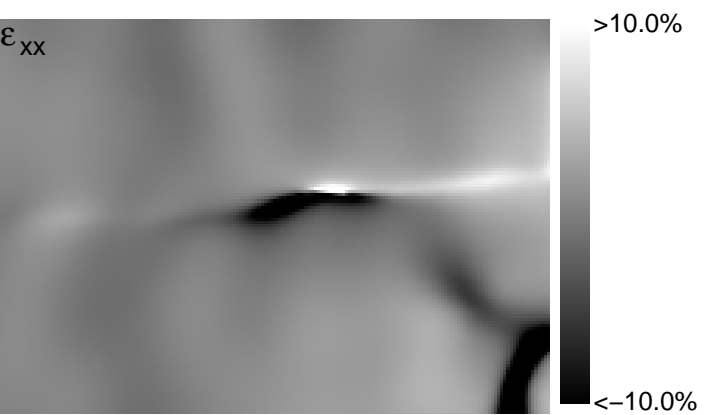

(c) $\omega_{x y}$

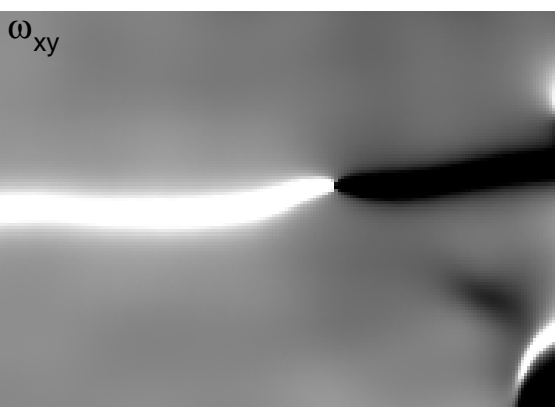

$>10.0 \%$

$(\mathrm{e}) \varepsilon_{x y}$

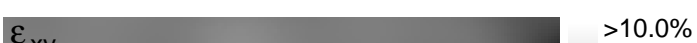

Abbildung 5.18: Partialversetzung zwischen zwei $\mathrm{I}_{1}$-Spiegelvarianten. In der unteren rechten Ecke ist der Probenrand zu erkennen. (a) Phasenrekonstruktion. Ein rechtsgewundener Burgersumlauf ist eingezeichnet, es ergibt sich $\boldsymbol{b}=\frac{1}{6}[1 \overline{1} 00]$. Die $x$ und $y$-Richtungen der GPA sind eingezeichnet. (b) Verschiebungsfeld in [11̄00]Richtung aus GPA, in [0002]-Richtung ist keine Verschiebung messbar. (c)-(e) Verzerrungsfelder $\epsilon_{x x}$ und $\epsilon_{x y}$ sowie Rotation $\omega_{x y}$ aus der GPA. Die GPA-Messungen zeigen den gleichen Ausschnitt wie die Phasenrekonstruktion. 
Knicks nicht eindeutig zu erkennen. Dies mag daran liegen, dass der PSF in dieser Projektion schräg in der Probe liegt. Im obereren Bereich läuft der BSF von rechts in den Ausschnitt und knickt dann nach unten in einen Drum-PSF ab, wobei er eine Stairrod-Versetzung mit dem Burgersvektor $\boldsymbol{b}=\frac{1}{6}[\overline{1} 100]$ hinterlässt. Im unteren Bereich des Auschnittes ist der PSF gut zu erkennen. Dass er vom Drum-Typ ist, lässt sich an drei Merkmalen erkennen: zum einen das Vorhandensein der Stairrod-Versetzung, die bei einem Blank-PSF nicht auftreten würde, zum anderen anhand des sechszähliges Muster, das charakteristisch für den Drum-Typ ist (s. Abschnitt 5.6), im Bereich des Stapelfehlers, wie auch in Abb. 5.19a eingezeichnet, und drittens durch die quantitative Messung des Verschiebungsfeldes mittels GPA. Es wird eine Verschiebung um $0.27 \cdot[1 \overline{1} 00]$ gemessen (s. Abb. 5.20), was dem Drum-Typ zugerechnet werden kann. Ein Blank-PSF würde eine Verschiebung von $\frac{1}{6}[1 \overline{1} 00]$ zeigen. Da dieser Defekt in der Nähe der Grenzfläche zum GaN-Template gefunden wurde, lässt sich die kleine Abweichung durch weitere Verschiebungsfelder aufgrund von Defekten in der Grenzfläche sowie durch eine mögliche Gitterrelaxation aufgrund der nahen Probenoberfläche erklären. 
(a)

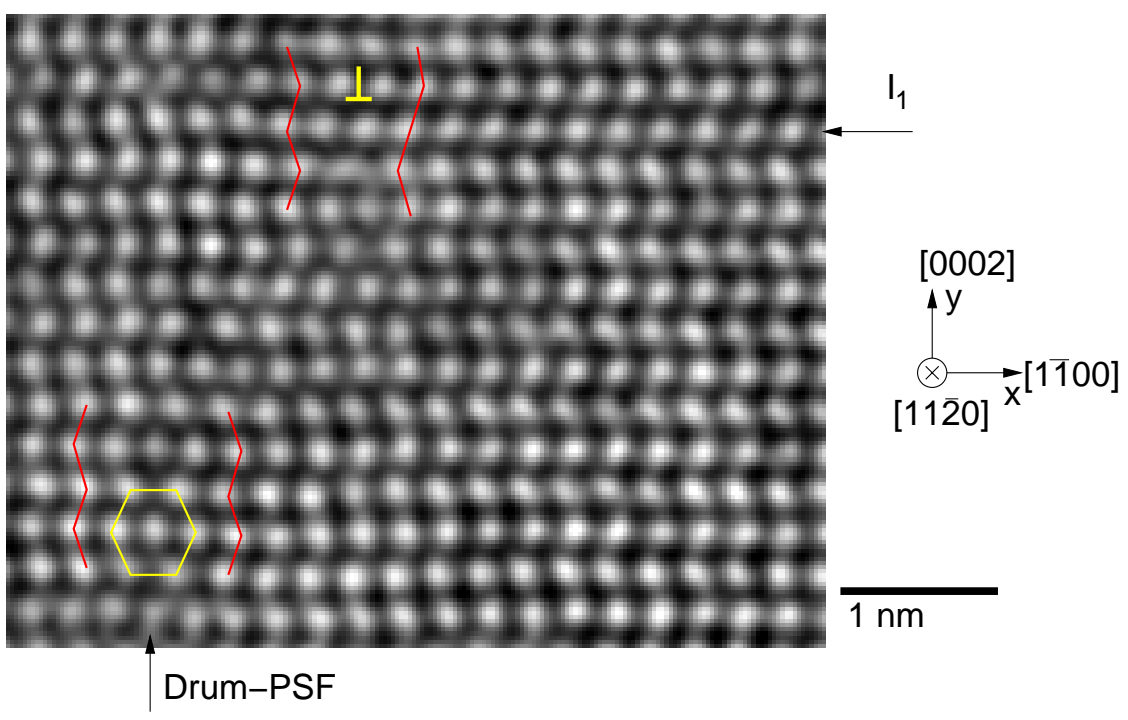

(b) $u_{x}$

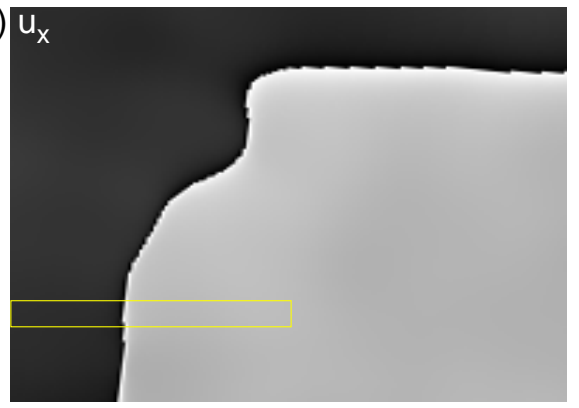

(d)

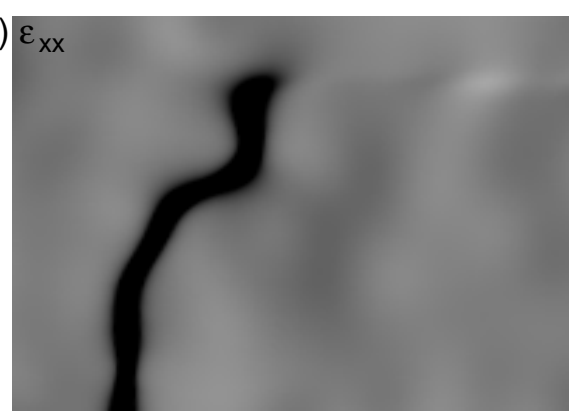

$0.137 \mathrm{~nm}$

$-0.137 \mathrm{~nm}$

$>10.0 \%$

(e) $\varepsilon_{x j}$

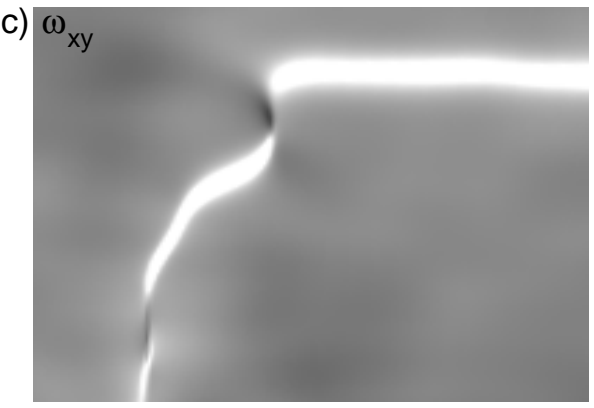

$\varepsilon_{x y}$

$>10.0 \%$

Abbildung 5.19: Partialversetzung zwischen einem basalen $I_{1}$-Stapelfehler und einem prismatischen Stapelfehler vom Drum-Typ. (a) Phasenrekonstruktion. Das für den Drum-PSF typische sechszählige Muster ist angedeutet. Die $x$ und $y$-Richtungen der GPA sind eingezeichnet. (b) Verschiebungsfeld in [1100]-Richtung aus GPA, in [0002]-Richtung tritt nur eine sehr kleine Verschiebung auf. (c)-(e) Verzerrungsfelder $\epsilon_{x x}$ und $\epsilon_{x y}$ sowie Rotation $\omega_{x y}$ aus der GPA. Die GPA-Messungen zeigen den gleichen Ausschnitt wie die Phasenrekonstruktion. 


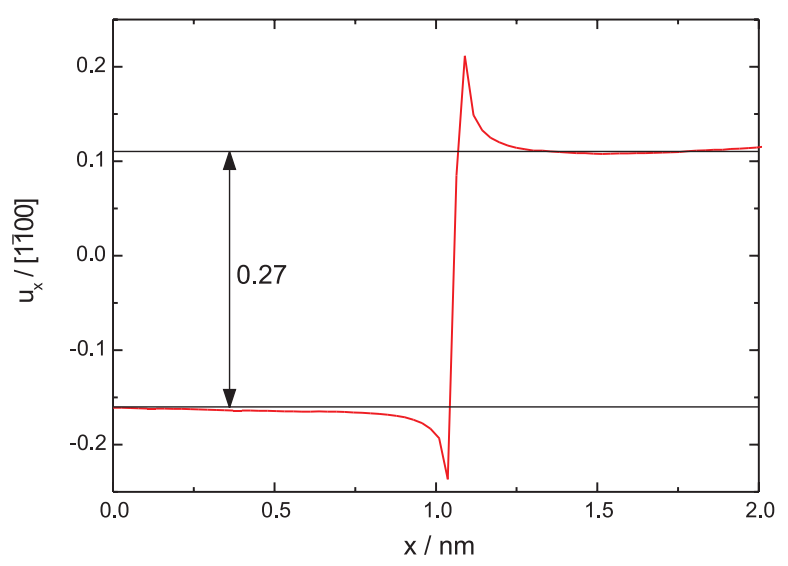

Abbildung 5.20: Verschiebungsprofil $\mathrm{u}_{x}$ aus dem in Abb. 5.19b gelb markierten Bereich. 


\section{Mn-Einbau in GaN}

\subsection{Ausscheidungen}

Es wurden Mn-Ausscheidungen in unter verschiedenen Wachstumsbedingungen gewachsenen Proben beobachtet: Die Probe J0087 wurde mit einem Mn-Angebot von 40 at\% und sehr stickstoffreich, die Proben J0120 und J0121 hingegen bei einem Mn-Angebot von 15\% und nahe am stöchiometrischen Punkt gewachsen. Während sich die Schichtmorphologien je nach Wachstumsbedingung unterscheiden, lässt sich doch in beiden Fällen die Phase der Ausscheidung als $\mathrm{GaMn}_{3} \mathrm{~N}$ identifizieren, wie im Folgenden gezeigt wird.

Abb. 6.1 zeigt Probe J0120 in einer HAADF (,high-angle annular dark field“) Abbildung. Bei diesem Dunkelfeld werden Bildanteile, die in einen großen Winkel gestreut wurden, zur Abbildung benutzt. Die Elektronen, die in solche Winkel gestreut wurden, haben vorwiegend (inkohärente) Rutherford-Streuung erfahren. Da der Rutherford-Streuquerschnitt eines einzelnen Atoms proportional zum Quadrat der Ordnungszahl ist, kann dieser so genannte Z-Kontrast verwendet werden, um chemische Unterschiede in den Proben sichtbar zu machen [57]. Eine Abschätzung der Streuquerschnitte der GaN-Matrix und der $\mathrm{GaMn}_{3} \mathrm{~N}-\mathrm{Ausscheidungen} \mathrm{ergibt}$ einen Unterschied von rund 30\%, was die gute Sichtbarkeit dieser Ausscheidungen erklärt. Neben dem Z-Kontrast sind in Abbildung 6.1 auch Beugungskontraste zu erkennen, die hier z.B. die Versetzungen hervorheben, d.h. das der HAADF-Kontrast kein reiner Z-Kontrast ist. In der unter vergleichbaren Bedingungen gewachsenen Probe J0121 findet sich die gleiche Schichtmorphologie.

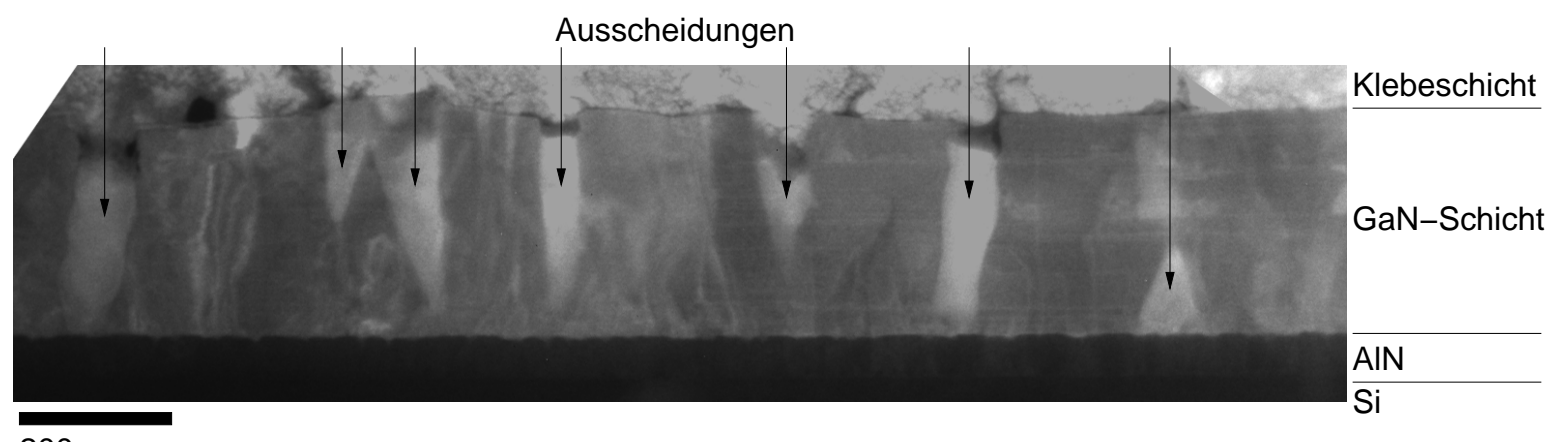

$200 \mathrm{~nm}$

Abbildung 6.1: Zusammengesetzte HAADF-Aufnahme einer GaN-Schicht mit Ausscheidungen (Probe J0120). Neben den Ausscheidungen, die sich durch ihren stärkeren Z-Kontrast herausheben, zeigen auch Kristallbaufehler (wie Stapelfehler und Versetzungen) Beugungskontraste, die der Schicht das leicht marmorierte Aussehen verleihen.

Auf der Probenoberseite haben die Ausscheidungen einen Durchmesser von rund $100 \mathrm{~nm}$, und ihr Abstand untereinander liegt im Bereich von $300 \mathrm{~nm}$. Diese Beobachtungen sind konsistent mit rasterelektronenmikroskopischen Aufsichtsaufnahmen dieser Probe (s. Abb. 6.2). 
Ein Teil dieser Ausscheidungen wird in Schichtwachstumsrichtung breiter. Die Oberfläche der Probe zeigt Vertiefungen am Ort der Ausscheidungen. Diese Vertiefungen sind auch in der Querschnittsaufnahme 6.1 als dunkler Kontrast zwischen den Ausscheidungen und der Klebeschicht zu erkennen. Diese Bereiche sind leer. Dies lässt sich den Schluss zu, dass in dieser Probe die GaN-Matrix im Vergleich zu den Ausscheidungen schneller wächst.

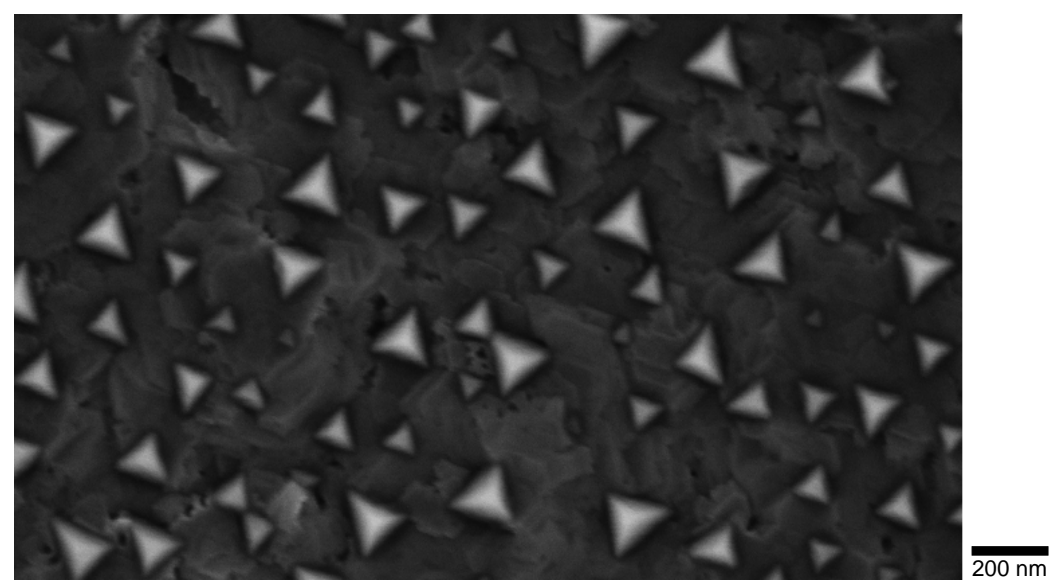

\begin{abstract}
Abbildung 6.2: REM-Aufsichtsaufnahme (Probe 120). Die Ausscheidungen haben eine dreieckige Grundfläche, ihr mittlerer Durchmesser liegt bei $100 \mathrm{~nm}$ und sie haben typische Abstände im Bereich von $300 \mathrm{~nm}$. Am Rand der Ausscheidungen ist ein dunkler Saum in der Matrix erkennbar. [Abbildung: M. Kocan]
\end{abstract}

Die HRTEM-Aufnahmen (Abb. 6.3) zeigen das untere Ende von Ausscheidungen, die bis an den Anfang des GaN herunterreichen. Hier ist nahe der Grenzfläche zur AlN-Pufferschicht, direkt am Fuß der Ausscheidungen, ein stark gestörtes Gitterbild zu sehen, das auf einen ausgedehnten Kristalldefekt, wahrscheinlich eine Versetzung mit Linienelement in $c$-Richtung, hindeutet. Auch zeigt das GaN auf beiden Seiten der Defekte ein unterschiedliches Muster, was von einem Verzerrungsfeld herrühren kann. Dies würde bedeuten, dass die Ausscheidungen an den Defekten keimen. Ausscheidungen, die in Querschnittsaufnahmen scheinbar in der Mitte der Schicht beginnen, keimen dann außerhalb des Querschnitts, so dass sie mit zunehmender Schichtdicke erst in den Querschnitt hineinwachsen.

In Abb. 6.4 ist eine Feinbereichsbeugungsaufnahme einer Ausscheidung aus dieser Probe gezeigt. Auf ihr sind die Beugungsreflexe zweier Gitter zu erkennen: zum einen das hexagonale GaN-Gitter (in blau dargestellt; Zonenachse [1120]) und zum anderen das kubische Gitter der Ausscheidung (in rot dargestellt; Zonenachse [110]). Aus der Lage der beiden Gitter relativ zueinander lässt sich eine epitaktische Beziehung für das Wachstum der Ausscheidungen ange-

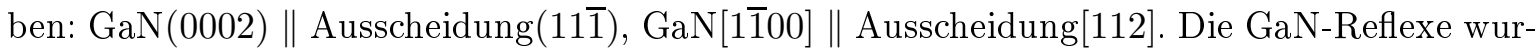
den benutzt, um die reziproken Längen in dem Beugungsbild zu kalibrieren. Für das kubische Gitter ergibt sich dann die Gitterkonstante $a=0.3922(40) \mathrm{nm}$. Die epitaktischen Beziehungen zum GaN und die Bestimmung der Gitterkonstanten ließen sich innerhalb des Fehlers in weiteren Beugungsbildern und Diffraktogrammen auch von anderen Proben bestätigen.

Im Materialsystem Ga-Mn-N ist eine einzige kubische Phase im Bereich der genannten Gitterkonstante bekannt [124]: $\mathrm{Ga}_{1-x} \mathrm{Mn}_{3+x} \mathrm{~N}$, dabei nimmt die Gitterkonstante dieser perowskitischen Phase monoton mit dem Ga-Gehalt zu von $a=0.3871 \mathrm{~nm}$ für $\mathrm{Mn}_{4} \mathrm{~N}$ bis $a=0.3885 \mathrm{~nm}$ 
(a)

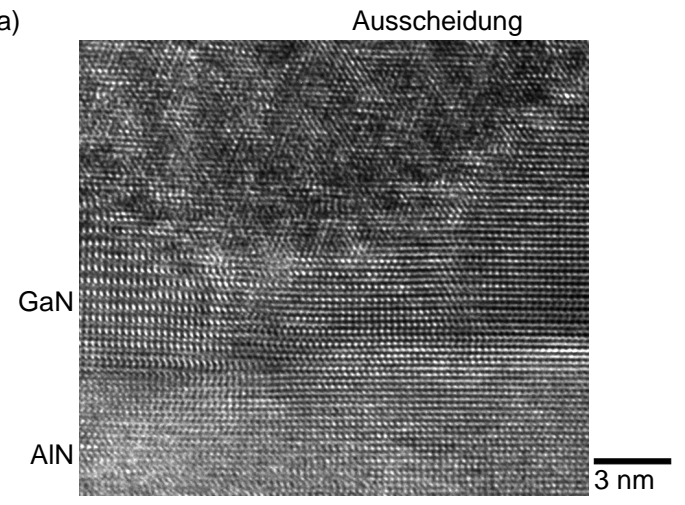

(b)

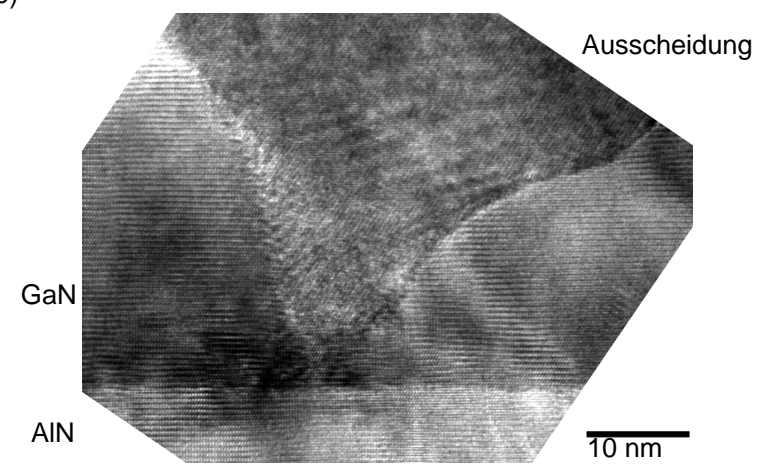

Abbildung 6.3: HRTEM-Aufnahme am unteren Ende der Ausscheidungen. Die Ausscheidungen keimen an Defekten in der Grenzschicht zum AlN. (a) Probe J0120. (b) Probe J0121.

für $\mathrm{GaMn}_{3} \mathrm{~N}$ [46]. Um die Ga-Konzentration dieser Phase zu bestimmen, wurde eine EDXAnalyse an den Ausscheidungen durchgeführt.

Die Spektren zweier solcher Messungen sind in Abb. 6.5 gezeigt. Die Messungen wurden in Probe J0121 durchgeführt, wobei das rote Spektrum von einer Ausscheidung und das blaue Spektrum von der GaN-Matrix stammt. Neben den Ga-, Mn- und N-Linien sind weitere Linien zu sehen, die als Messartefakte erklärt werden können: Während der Probenpräparation durch Ionenätzen wurden auch geringe Teile des Probenträgers $(\mathrm{Cu})$ und des Substrats $(\mathrm{Si})$ auf die Probe aufgesputtert, die in der Messung sichtbar werden. Ebenso kontaminieren die Probenoberflächen an Luft mit Sauerstoff, und unter Elektronenbestrahlung im Mikroskop mit Kohlenstoff. Zur Konzentrationsbestimmung werden die Intensitäten der Ga-K- und MnK-Linien bestimmt. Die N-Linie ist aus Gründen, die in Abschnitt 7.3 beschrieben werden, nicht für eine quantitative Messung geeignet.

Das Ergebnis der EDX-Analyse einer Ausscheidung in Probe J0121 ist in Abb. 6.6 gezeigt. In der Ausscheidung finden sich Ga und Mn im Verhältnis 1:3, was die Ausscheidung als $\mathrm{GaMn}_{3} \mathrm{~N}$ identifiziert. Der Mn-Gehalt der Matrix sinkt auf unter 1\%, was von weiteren EDX-Messungen bestätigt wird, die weiter entfernt vom Rand der Ausscheidung vorgenommen wurden. Somit befindet sich das $\mathrm{Mn}$ in dieser Probe im Wesentlichen in den Ausscheidungen.

Die Probe wurde bei $650^{\circ} \mathrm{C}$ gewachsen und das Wachstum der gesamten Schicht hat 3 Stunden benötigt. Die Probe wurde danach nicht ausgelagert. Um zu beurteilen, ob sich das Mn im Volumen durch Diffusion umverteilt hat, oder ob diese Umverteilung bereits während des Wachstum an der Oberfläche der Schicht stattgefunden hat, soll die Diffusionslänge von Mn im Volumen abgeschätzt werden. Cai et al. [125] haben die Diffusionskonstante von Mn in $\mathrm{GaN}$ aus Eindiffusionsexperimenten für $500^{\circ} \mathrm{C}$ und $800^{\circ} \mathrm{C}$ bestimmt. Mit der Annahme, dass sich die Diffusionskonstante wie ein Arrhenius-Term verhält, lässt sich daraus für die Wachstumstemperatur interpolieren:

$$
D\left(650^{\circ} \mathrm{C}\right)=2.07 \cdot 10^{-16} \frac{\mathrm{cm}^{2}}{\mathrm{~s}} .
$$

Mit der Beziehung $x=2 \sqrt{D t}$ ergibt sich damit eine Diffusionslänge von $17 \mathrm{~nm}$ für 1 Stunde (30 nm bei 3 Std.). Da bei einem Abstand der Ausscheidung untereinander von bis zu $300 \mathrm{~nm}$ 
(a)

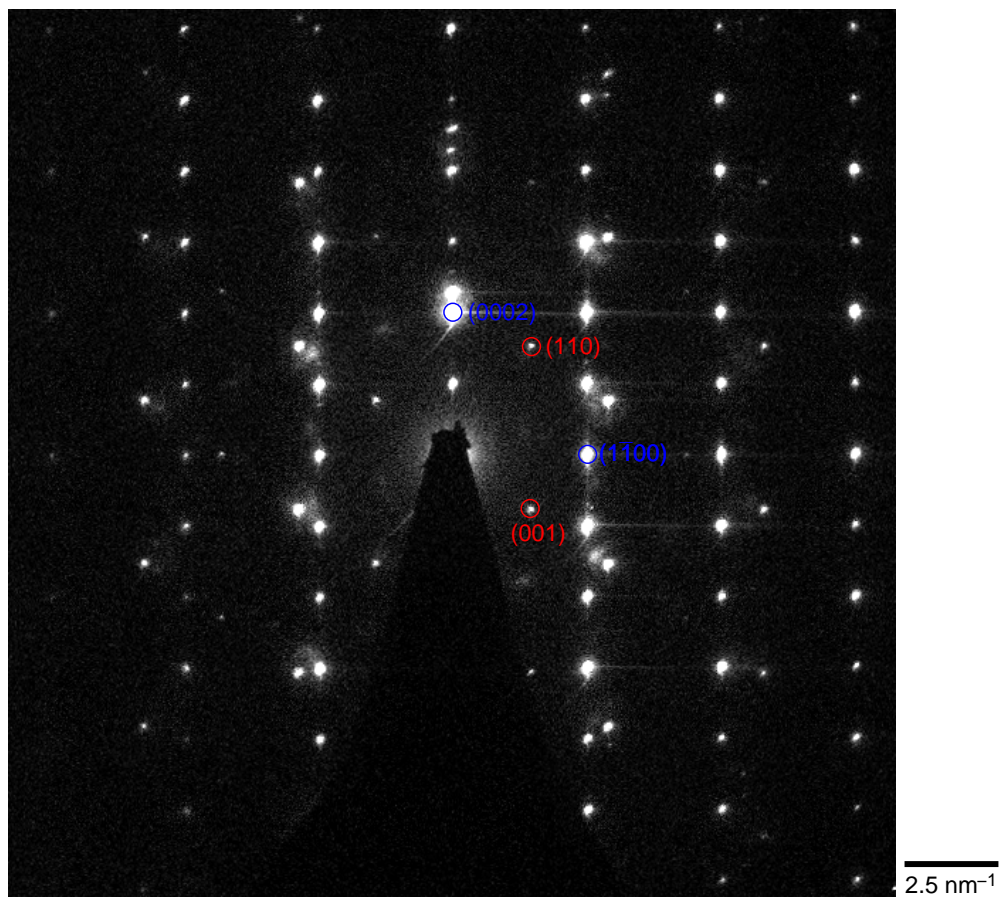

(b)

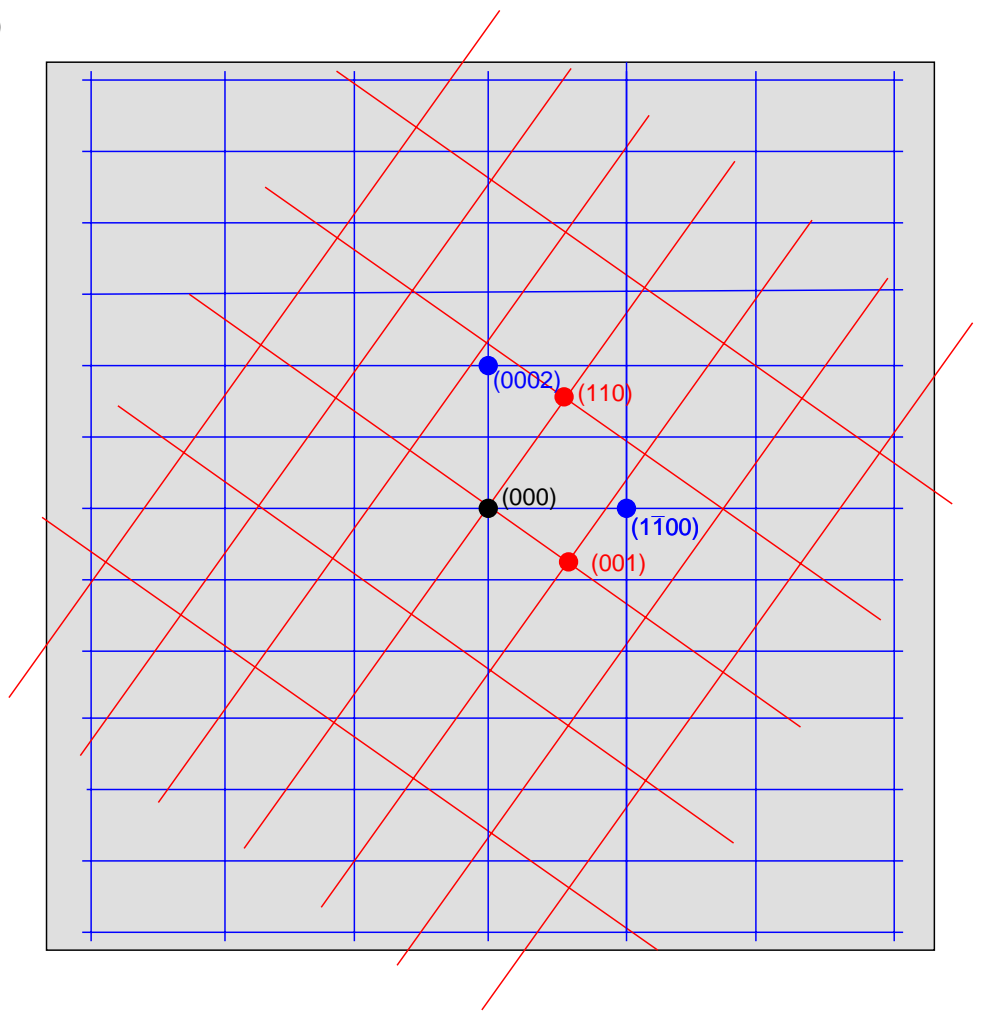

Abbildung 6.4: (a) Beugungsbild von GaN mit GaMn ${ }_{3} \mathrm{~N}-$ Ausscheidung (logarithmische Skala, Modulationstransferfunktion entfaltet). Die Schweife der intensiven Reflexe sind Artefakte der Aufnahme. (b) Schema der reziproken Gitter mit je zwei grundlegenden Reflexen, GaN in Blau, $\mathrm{GaMn}_{3} \mathrm{~N}$ in Rot. Die weiteren zugehörigen Reflexe befinden sich auf den Kreuzungspunkten der Gitter. 


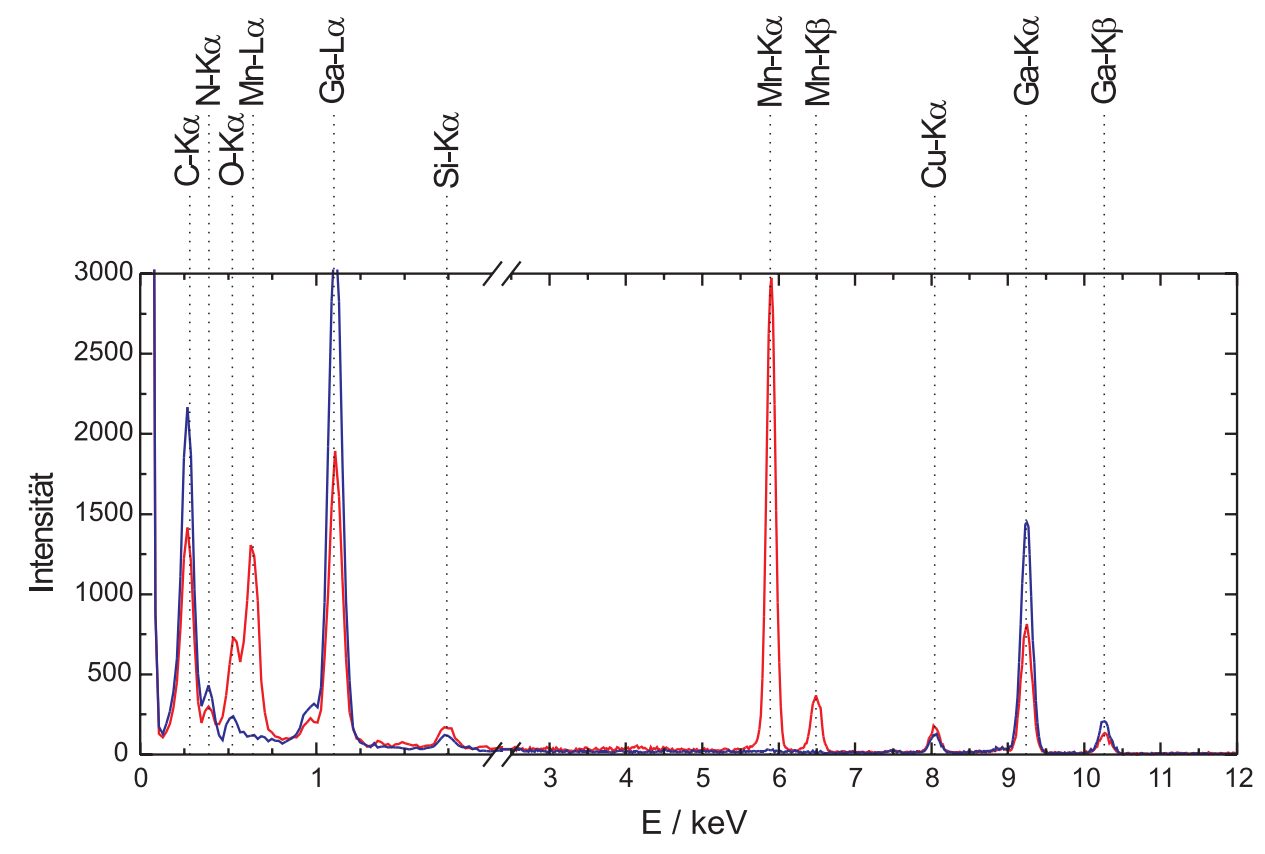

Abbildung 6.5: EDX-Spektrum. Die Cliff-Lorimer-Auswertung (s. Abschnitt 2.2.2) des blauen Spektrums ergibt ein Mn/Ga-Verhältnis von 0.5 zu 99.5 at\%, d.h. das Mn ist unter der Nachweisgrenze. Die Auswertung des roten Spektrums ergibt ein Verhältnis von $25.5 \mathrm{zu} 74.5$ at\%.

(a)

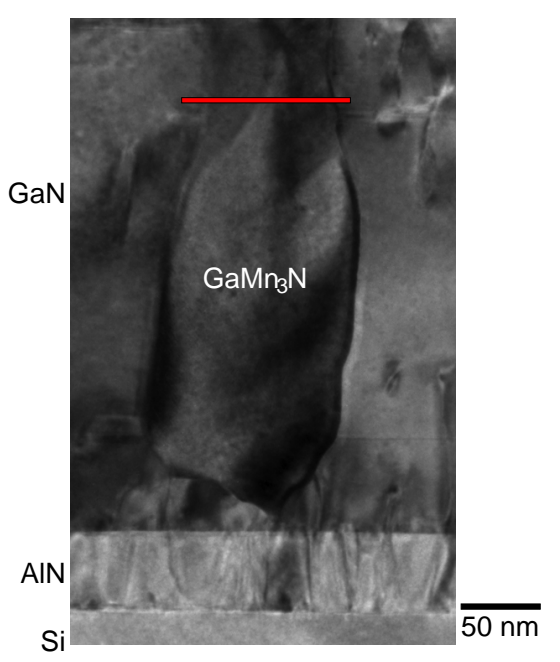

(b)

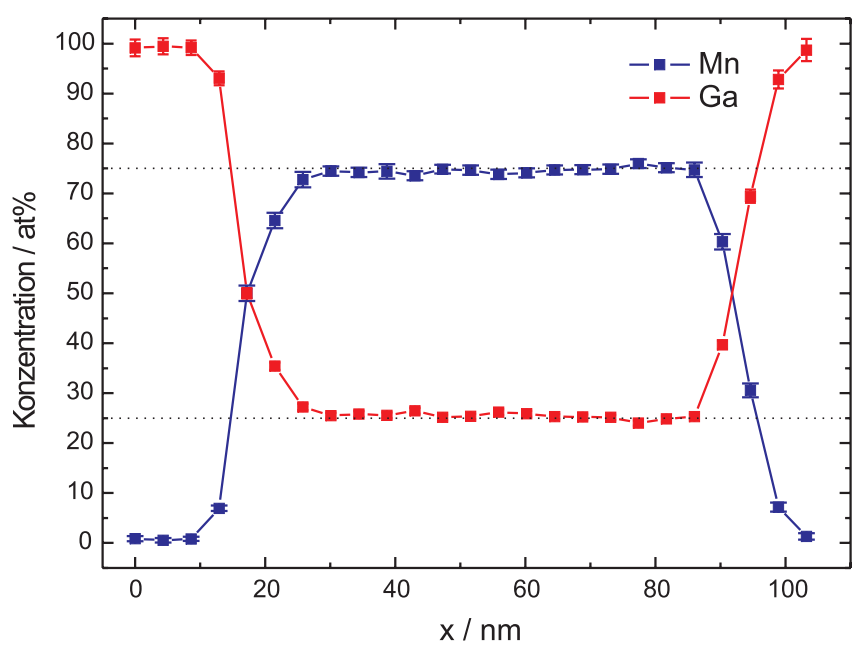

Abbildung 6.6: EDX-Analyse einer Ausscheidung. (a) Hellfeldaufnahme der untersuchten Ausscheidung. Die EDX-Messung wurde entlang der roten Linie durchgeführt. (b) Ergebnis der Konzentrationsbestimmung: die Ausscheidung enthält Mn und Ga im Verhältnis 1:3. Die Linien dienen der Orientierung. 
Längen von rund $150 \mathrm{~nm}$ zurückgelegt werden müssen, kann somit geschlossen werden, dass die Ausscheidungsbildung durch Diffusionsprozesse an der Oberfläche statt gefunden haben muss.

Aus den Beobachtungen lässt sich die Diffusionskonstante des Mn während des Wachstums an der Oberfläche abschätzen. Dazu wird angenommen, dass das Mn nur an der Oberfläche diffundieren kann. Aus der GaN-Schichtdicke von $300 \mathrm{~nm}$ ergibt sich, dass alle $9.34 \mathrm{~s}$ eine GaN-Doppelebene, d.h. ein GaN-Molekül pro Einheitszellenfläche, deponiert wurde. In dieser Zeit mussten die Mn-Atome bis zu $150 \mathrm{~nm}$ zurücklegen, so dass sich nach obiger Beziehung für die Diffusionslänge die Diffusionskonstante auf mindestens

$$
D=6.0 \cdot 10^{-12} \frac{\mathrm{cm}^{2}}{\mathrm{~s}}
$$

abschätzen lässt.

Eine Hellfeld-Aufnahme aus Probe J0087 ist in Abb. 6.7 gezeigt. Im Unterschied zu den nahe am stöchiometrischen Verhältnis gewachsenen Proben J0120 und J0121 ist das GaN hier in einer stärker gestörten Schicht gewachsen. Die Ausscheidungen scheinen wieder bis zur AlN-Grenzschicht hinabzureichen, so reicht das Moiré-Muster, das durch die Überlagerung des Gitters der Ausscheidung mit dem Gitter der Matrix entsteht, bis an die Grenzfläche heran. Die Ausscheidungen dieser Probe scheinen aus der Oberfläche herauszuwachsen, ihre Durchmesser variieren im Bereich von 50-100 nm und die typischen Abstände liegen zwischen 50 und 200 nm. Letzteres bedeutet gegenüber Probe J0120 eine höhere Dichte von Ausscheidungen, was aufgrund des höheren Manganangebots während des Wachstums auch zu erwarten war.

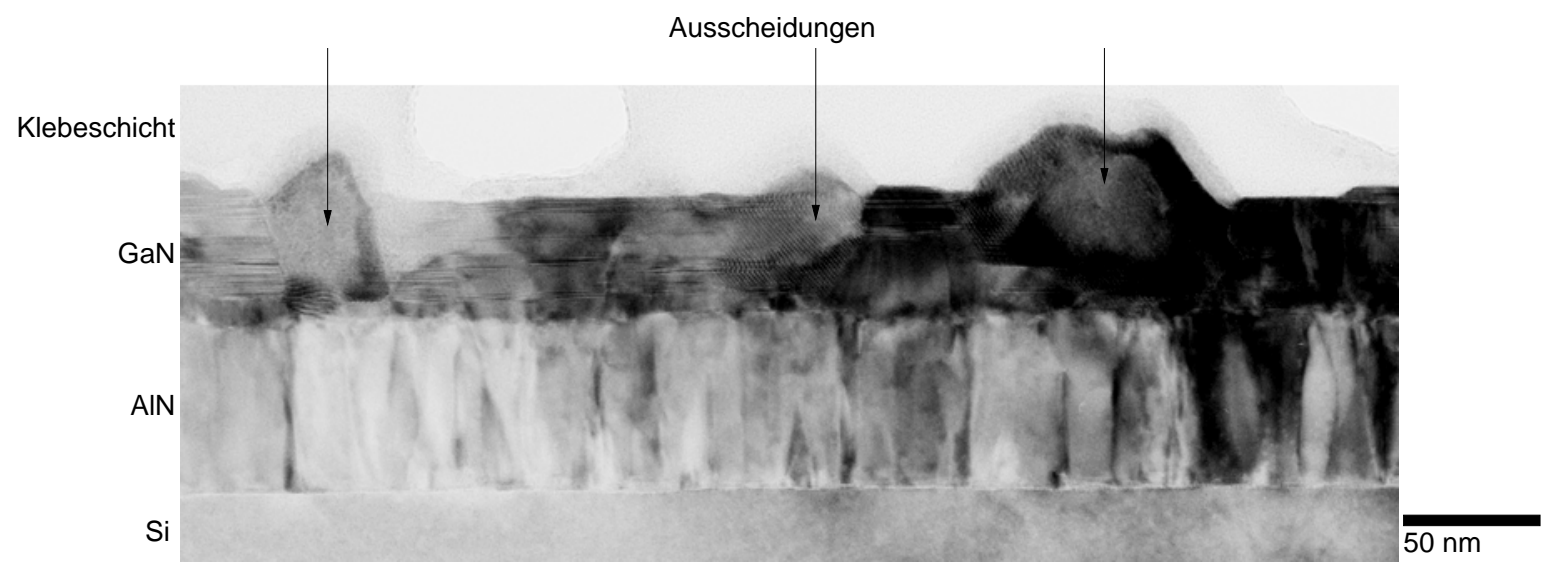

Abbildung 6.7: Hellfeldaufnahme von Ausscheidungen einer unter stickstoffreichen Bedingungen gewachsenen Probe (J0087). Die Streifenmuster an der Grenzfläche der Ausscheidungen zur GaN-Matrix sind MoiréEffekte der beiden Kristallgitter.

Die für diese Probe beobachtete Größen- und Abstandsverteilung ist konsistent mit Beobachtungen aus REM-Aufsichtsaufnahmen (s. Abb. 6.8). Der Umstand, dass in dieser Probe die Ausscheidungen aus der Schicht herausragen, während sie in der Probe J0120 Vertiefungen in der Oberfläche darstellen, könnte den unterschiedlichen Kontrast am Rande der Ausscheidungen erklären, der in den REM-Aufnahmen zu beobachten ist: In der Aufnahme aus Abb. 6.2 (Probe J0120) ist ein dunkler Saum in der Matrix am Rand der Ausscheidungen zu erkennen, 
diese Säume sind nicht in Abb. 6.8 zu finden. Die Abbildungsbedingungen (Beschleunigungsspannung, Arbeitsabstand) waren bei beiden Aufnahmen identisch. Das große Mn-Angebot (40 at\%) in dieser Probe führt scheinbar zu einem gegenüber den Ausscheidungen langsameren Wachstum der GaN-Matrix.

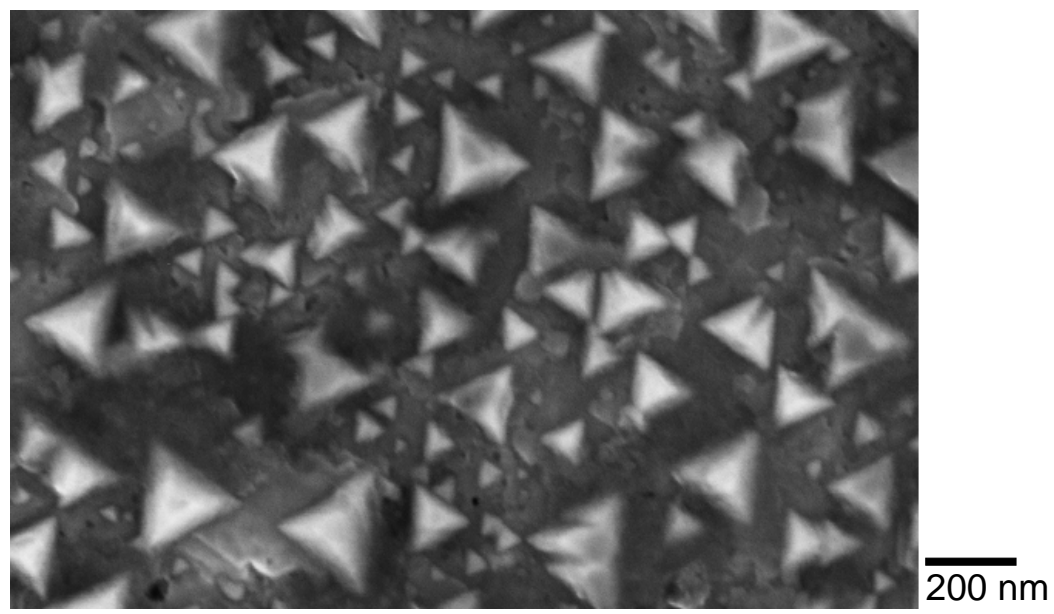

\begin{abstract}
Abbildung 6.8: REM-Aufsicht der Probe J0087. Die Ausscheidungen zeigen eine Größenverteilung von 50-200 nm und eine Abstandsverteilung im Bereich 50-200 nm. Ein deutlicher dunkler Saum um die Ausscheidungen, wie in Abb. 6.2 erkennbar, ist nicht sichtbar. [Abbildung: M. Kocan]
\end{abstract}

Bestimmung der Gitterkonstante aus Diffraktogrammen von HRTEM-Aufnahmen (hier nicht gezeigt) ergab für die Ausscheidungen aus Probe J0087 $a=0.3877(18) \mathrm{nm}$ und die bereits oben genannten epitaktischen Beziehungen zwischen Ausscheidung und Matrix. Durch EDXAnalysen wurde auch in diesen Ausscheidungen ein Ga/Mn-Verhältnis von 1:3 nachgewiesen. Daher werden auch diese Ausscheidungen als $\mathrm{GaMn}_{3} \mathrm{~N}$ identifiziert.

An den Oberflächen der Ausscheidungen wurde zusätzlich eine dünne Schicht $(\approx 10 \mathrm{~nm})$ einer weiteren polykristallinen Phase entdeckt, die allerdings nicht eindeutig identifiziert werden konnte. Anhang $\mathrm{C}$ befasst sich mit dieser Phase. Im Volumen der Proben wurden ansonsten keine Ausscheidungen einer anderen Phase beobachtet.

\title{
6.2 Gelöstes Mn
}

In Abbildung 6.9 sind Übersichtsaufnahmen von Probe J0141 gezeigt, die auf einem GaNTemplate gewachsen wurde. In dieser Probe wurden keine Ausscheidungen gefunden. Augenfällig ist, dass das GaN nicht als geschlossene Schicht sondern säulenartig wächst. Die Säulen haben einen Durchmesser im Bereich von 50-100 nm.

Dieses säulenartige Wachstum wird durch das Mn verursacht: Zum Vergleich ist in Abb. 6.10 eine Aufnahme einer Schicht gezeigt (Probe J0145), die unter den gleichen Bedingungen wie Probe J0141 gewachsen wurde, d.h. bei gleicher Wachstumstemperatur und bei gleichem Gesamtmetall-Fluss, allerdings ohne Mn. Diese Probe weist eine geschlossene GaN-Schicht auf. Es ist bekannt, dass unter sehr stickstoffreichem Wachstumsbedingungen ein derartiges 


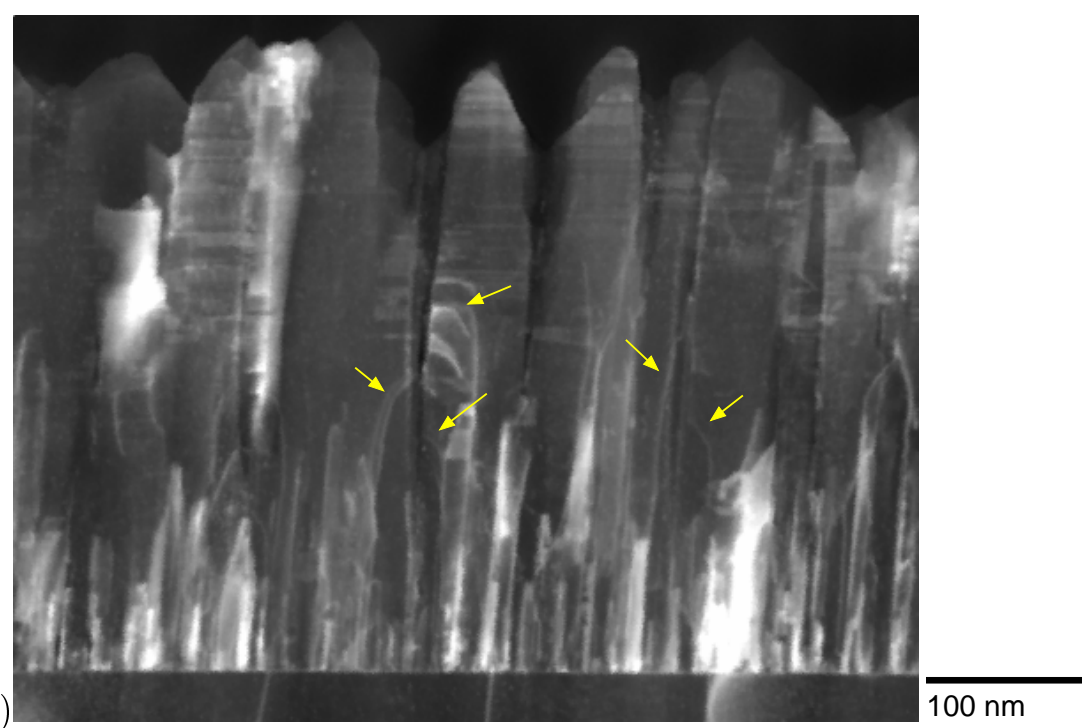

(a)

$100 \mathrm{~nm}$

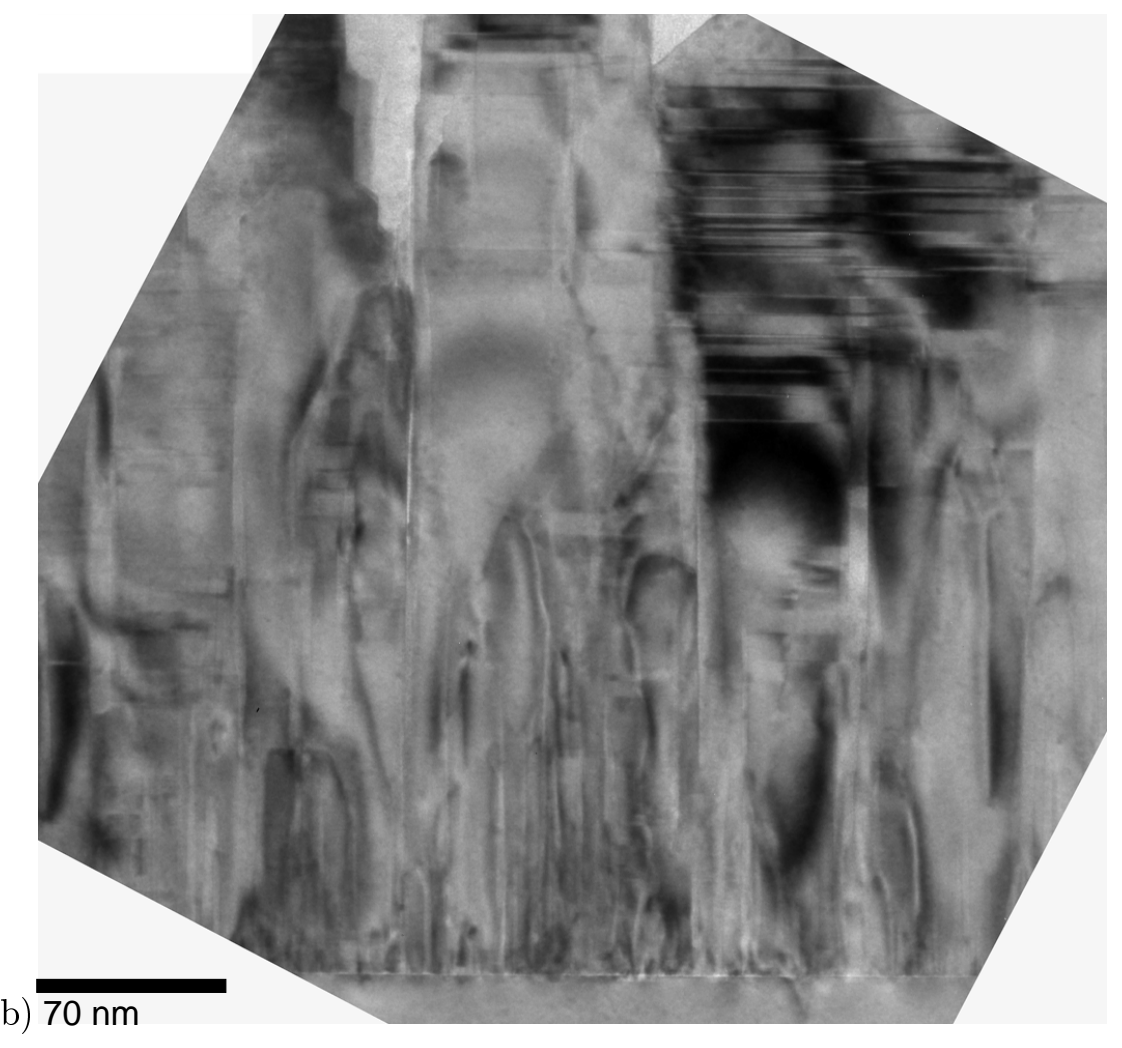

Abbildung 6.9: Übersicht über die GaN:Mn-Schichten der Probe J0141. Am unteren Rand der Abbildungen ist das GaN-Template zu erkennen: (a) STEM-Dunkelfeld, die leichte Scherung ist ein Artefakt der Aufnahme. Die Pfeile zeigen Fadenversetzungen, die bereits in der Mitte der Schicht an den Säulenoberflächen die Probe verlassen. (b) TEM-Hellfeld. 
Säulenwachstum auftritt, da der Stickstoff die Adatom-Diffusion des Ga an der Oberfläche behindert [126, 127]. Da das Säulenwachstum allerdings nicht in der Probe ohne Mn auftritt, kann geschlossen werden, dass die es nicht die stickstoffreichen Bedingungen allein sind, die das Säulenwachstum verursachen, sondern dass das Mn ebenfalls einen derartigen Einfluss auf das Wachstum haben muss.

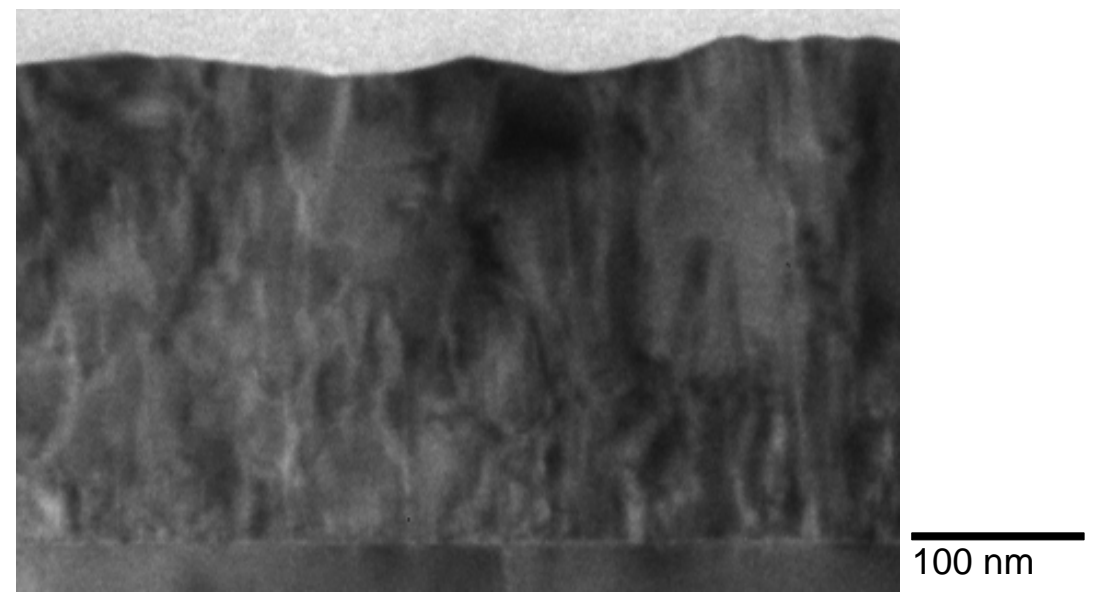

\begin{abstract}
Abbildung 6.10: Hellfeldaufnahme von Probe J0145, die unter identischen Bedingungen wie Probe J0141
\end{abstract} gewachsen wurde, allerdings ohne Mn. Am unteren Rand der Aufnahme ist das GaN-Template zu erkennen.

In den Säulen lassen sich viele Defekte auf Basalebenen erkennen, die in Abschnitt 5.3 als intrinsische Stapelfehler identifiziert wurden. Auch ließ sich keine signifikante Änderung der Mn-Konzentration mittels EDX im Bereich dieser Stapelfehler feststellen. Aufgrund der hohen Versetzungsdichte im unteren Teil der Säulen sind hier diese Stapelfehler schwer zu erkennen. Durch die Oberflächen der Säulen ergeben sich für Fadenversetzungen bereits nahe am Schichtbeginn Möglichkeiten aus der Probe auszutreten. Dies lässt sich in Abb. 6.9a am Abknicken dieser Versetzungen zum Rand der Säulen hin erkennen, wie mit Pfeilen markiert ist. Ausscheidungen sind in dieser Probe nicht nachzuweisen.

Das säulenartige Wachstum ist auch in Aufsichtsaufnahmen dieser Schicht sichtbar (Abb. 6.11). Der Säulendurchmesser von etwa $100 \mathrm{~nm}$ lässt sich auch hier wiederfinden. Die Winkel zwischen diesen Facetten betragen Vielfache von $30^{\circ}$. In Hochauflösungsaufnahmen ließen sich diese Facetten als die prismatischen $\{11 \overline{2} 0\}$ - und $\{1 \overline{1} 00\}$-Ebenen identifizieren. Die Säulen sind dabei teilweise zusammengewachsen. Zwischen nicht zusammengewachsenen Säulen finden sich leere Bereiche, teilweise mit Breiten bis unterhalb von $10 \mathrm{~nm}$, die auch in Querschnittsaufnahmen beobachtet werden können. Die Kontraste zwischen den Säulen in Abb. 6.11 stammen vom GaN-Template und von während der Präparation aufgesputtertem Material.

Für den Querschnitt in Abb. 6.12a ist eine chemische Analyse mittels EDX durchgeführt worden. Dabei wurden die Zählereignisse für die einzelnen Röntgenlinien der verschiedenen Elemente für jeden Punkt des Bildes gesondert gezählt. Dies ermöglicht jedoch aufgrund der mangelnden Hintergrundkorrektur der Röntgen-Intensitäten und der schlechten Zählstatistiken nur qualitative Aussagen. Da unterschiedliche Zählraten auch durch eine unterschiedliche Probendicke auftreten können, wurden die Intensitäten in jedem Punkt mit der Summe der Intensitäten der Ga-K $\alpha$-Linie und Mn-K $\alpha$-Linie skaliert. Die Summe ist proportional zur Probendicke. In Abb. 6.12b ist das skalierte Mn-Verhältnis gezeigt. Da im oberen Bildbereich 


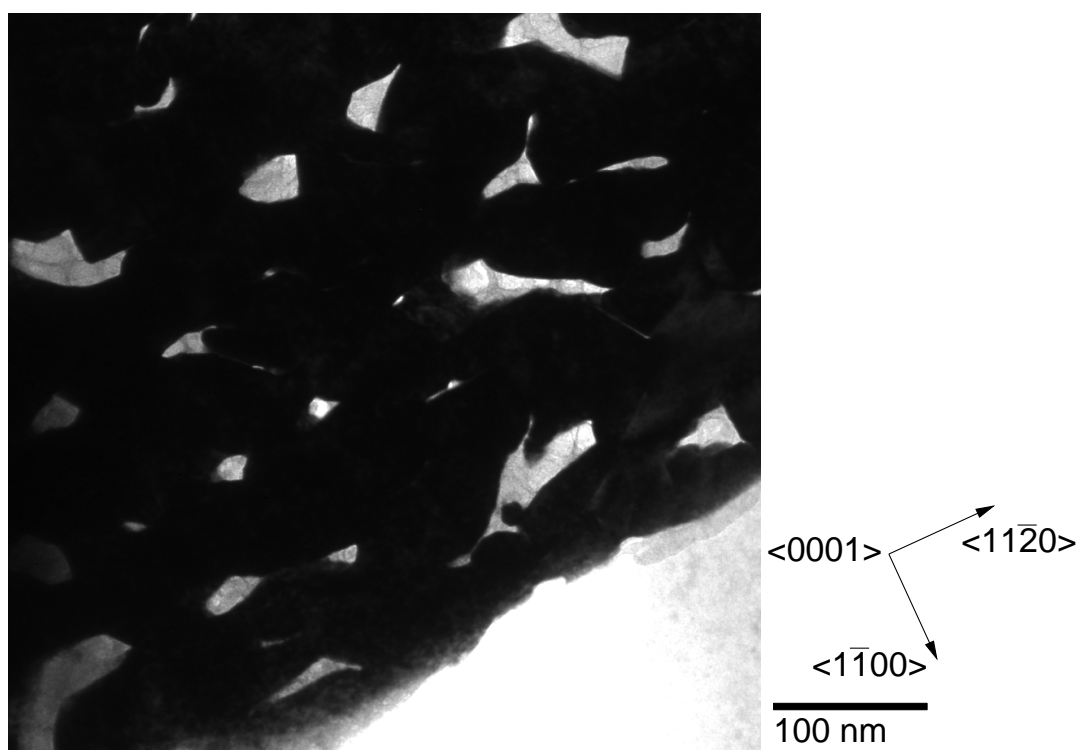

Abbildung 6.11: Aufsichtaufnahme von Probe J0141. Die Säulen haben einen Durchmesser im Bereich von $100 \mathrm{~nm}$ und sind mit prismatischen Ebenen facettiert. Zwischen den nicht zusammengewachsenen Säulen finden sich leere Bereiche.

Vakuum und die Klebeschicht sichtbar sind, wird hier das Hintergrundrauschen aufgrund der Skalierung verstärkt. Um diesen störenden Effekt zu beseitigen, sind Bildpunkte mit weniger als 35 Ga-Zählereignissen (Maximum bei 290) schwarz gefärbt. Die scheinbar hohen MnKonzentrationen am oberen Probenrand können ebenfalls von dieser Skalierung herrühren.

Es lässt sich erkennen, dass das Mn nicht homogen gelöst ist. Es ist (insbesondere im oberen Bereich der Schicht) vorwiegend in der Mitte der Säulen gelöst. Dies lässt sich insbesondere in den für Punktmessungen markierten Säulen erkennen. Die anderen Säulen liegen in dieser Projektion teilweise übereinander, was die Interpretation der Messung dort erschwert. Es ist auch erkennbar, dass die Mn-Anreicherung nicht in den Bereichen der im STEM-Bild erkennbaren Fadenversetzungen stattfindet.

Um zusätzlich quantitative Ergebnisse für die Mn-Verteilung zu erhalten, wurden an den in Abb. 6.12b eingezeichneten Stellen EDX-Punktmessungen durchgeführt. Sie ergaben folgende Zusammensetzungen (in at\%; bezogen auf den Gesamtmetall-Anteil):

\begin{tabular}{ccccccc} 
& $\mathrm{A}$ & $\mathrm{B}$ & $\mathrm{C}$ & $\mathrm{D}$ & $\mathrm{E}$ & $\mathrm{F}$ \\
\hline \hline $\mathrm{Mn}$ & 0.49 & 1.60 & 0.29 & 0.17 & 4.64 & 0.24 \\
$\mathrm{Ga}$ & 99.51 & 98.40 & 99.71 & 99.83 & 95.36 & 99.76 \\
\hline
\end{tabular}

Die mittlere Mn-Konzentration der Schicht lag bei 1.5 at\%.

Diesen Effekt der Mn-Anreicherung in der Mitte der Säulen lässt sich auch in Aufsichtsaufnahmen aus dem oberen Teil der Schicht (s. Abb. 6.13) beobachten. Auch hier wurden wieder zur besseren Sichtbarkeit, diejenigen Bildpunkte mit weniger als 90 Zählergeignissen für die 
(a)

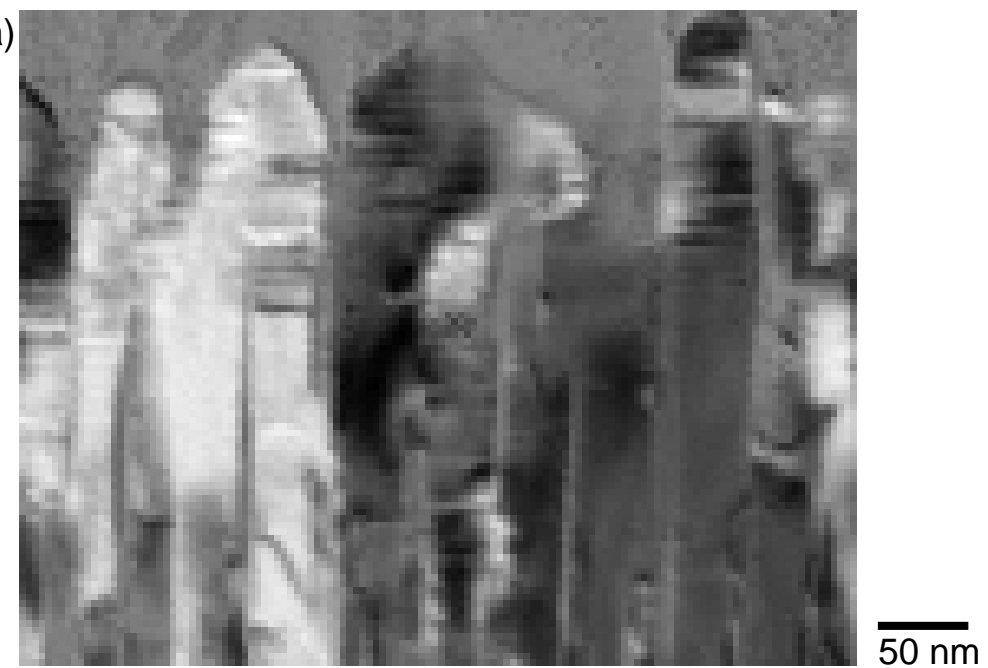

(b)

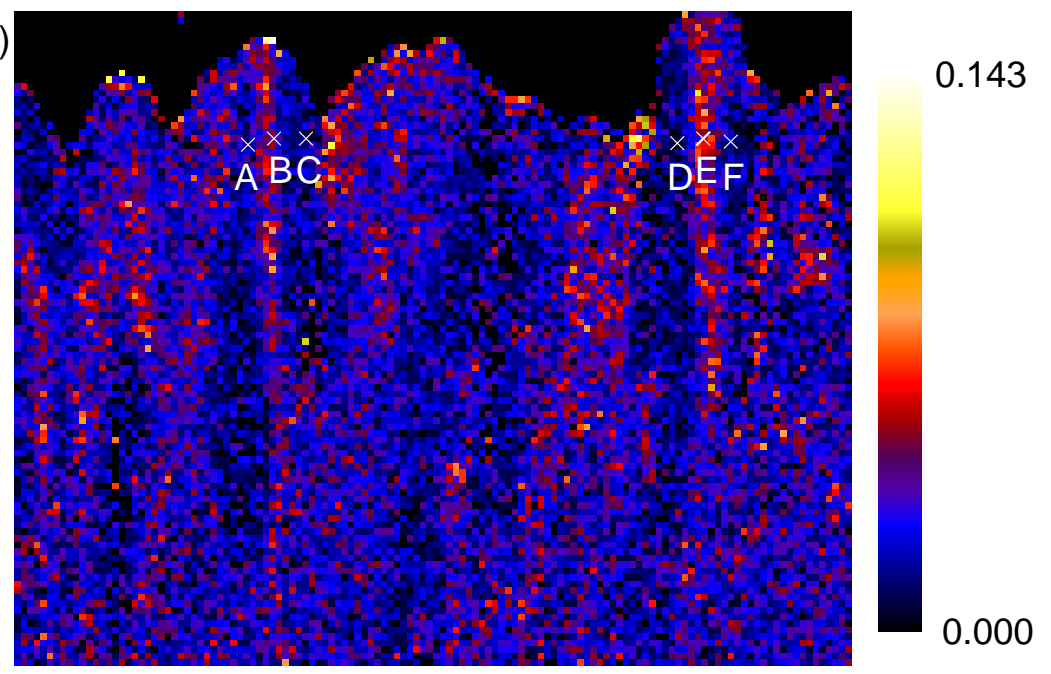

Abbildung 6.12: (a) STEM-Aufnahme. (b) EDX-Messung: das Verhältnis der Zählraten der Mn-K $\alpha$-Linie zur Summe der Mn-K $\alpha$ und Ga-K $\alpha$-Linien ist aufgetragen. Zur besseren Sichtbarkeit wurden die Punkte mit weniger als 35 Ga-Zählereignissen schwarz gefärbt. Die Buchstaben bezeichnen die Orte der Punktmessungen (s. Text). 
(a)

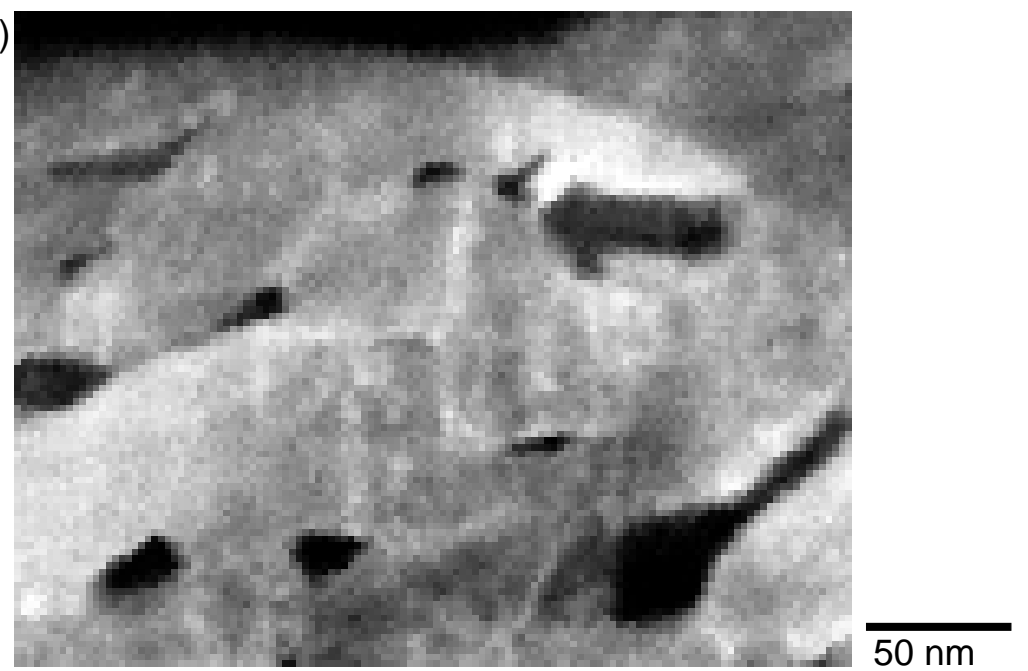

(b)

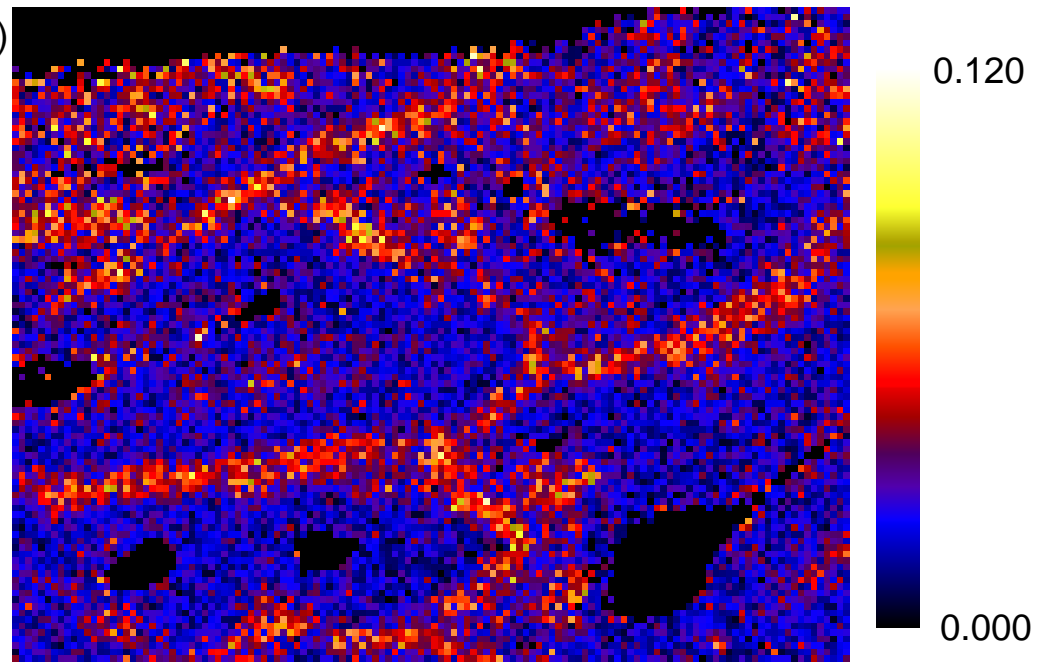

Abbildung 6.13: (a) STEM-Aufnahme. (b) EDX-Messung: das Verhältnis der Zählraten der Mn Ko-Linie zur Summe der Mn- und Ga-K $\alpha$-Linien ist aufgetragen. Zur besseren Sichtbarkeit wurden die Punkte mit weniger als 90 Ga-Zählereignissen schwarz gefärbt. 
Ga-K $\alpha$-Linie (Maximalwert 319) schwarz gefärbt. Trotzdem ist am oberen Rand das Rauschen aufgrund der geringen Probendicke sichtbar.

In Abb. 6.13b ist zu beobachten, dass das Mn in der Mitte der Säulenreihen angereichert ist. Es findet sich in den von den Seitenflächen am weitesten entfernten Bereichen der Säulen. Es lässt sich durch diese Messung ausschließen, dass sich das Mn in dieser Probe ausschließlich im Bereich von Kristalldefekten wie den Kleinwinkel-Korngrenzen zwischen den Säulen oder gar in Fadenversetzungen ansammelt. Dies steht im Einklang mit den Beobachtungen aus der Querschnittsprobe, in denen ebenfalls keine Korrelation zwischen Mn-Anreicherung und Kristalldefekten erkennbar war.

In Abb. 6.14 ist eine Übersichts-Hellfeldaufnahme von Probe J0091 gezeigt. Diese Probe wurde unter sehr stickstoffreichen Bedingungen und bei einem Mn-Angebot von 15\% gewachsen. Das GaN wächst in dieser Probe zunächst als zusammenhängende, sehr defektreiche Schicht. Im weiteren Verlauf des Probenwachstums, wächst diese Schicht nicht geschlossen weiter, sondern innerhalb von 50-100 nm großen Bereichen, mit geringerer Defektdichte als in der anfänglichen Schicht. Nur sehr vereinzelte, wenige Nanometer große Ausscheidungen wurden in dieser Probe gefunden.

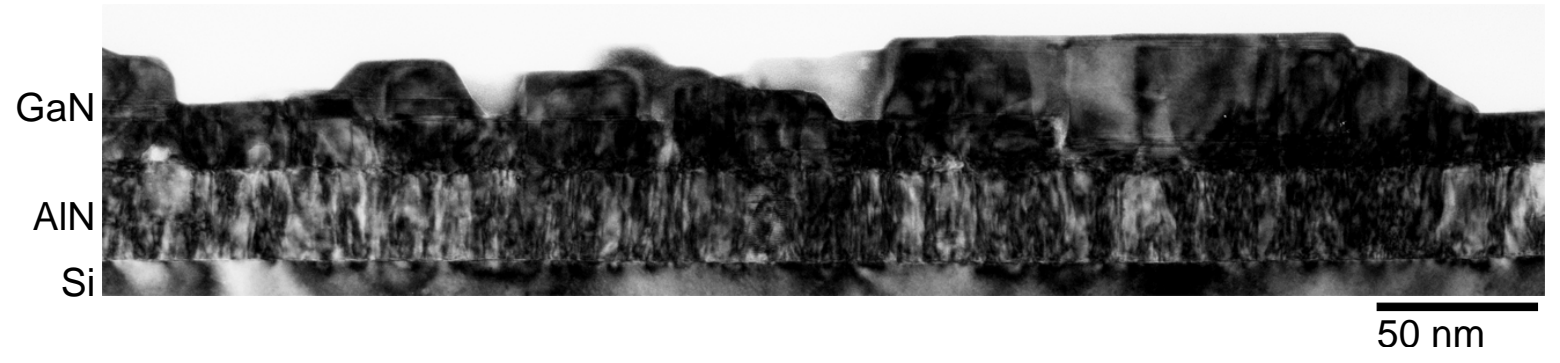

Abbildung 6.14: Hellfeld-Aufnahme von Probe J0091.

Zur Untersuchung der Menge des eingebauten Mangans und seiner Verteilung innerhalb der Probe wurden, wie schon in Probe J0141, EDX-Untersuchungen durchgeführt. In Abb. 6.15b ist das Mn-K $\alpha$-Signal im Verhältnis zur Summe der $\mathrm{K} \alpha$-Linien der Metall-Signale für den Auschnitt aus Abb. 6.15a gezeigt (Punkte mit weniger als 50 Zählereignissen sind schwarz gefärbt). Wie schon in Probe J0141 ist auch hier eine ungleichmäßige Verteilung des Mn zu erkennen.

An den Grenzen der Bereiche mit höherer Mn-Konzentration zu Bereichen mit niedrigerer Konzentration lassen sich in dieser Probe Kristalldefekte finden. Dies wird im Vergleich der EDX-Messung in Abb. 6.15 mit der Dunkelfeldaufnahme dieser Probenstelle in Abb. 6.16 deutlich. Der mit A gekennzeichnete Bereich hat eine geringere Mn Konzentration. An seinem oberen und unteren Ende finden sich ausgedehnte basale Defekte (wahrscheinlich Stapelfehler). An den mit B und C gekennzeichneten Stellen findet sich ein prismatischer Defekt. Diese Defekte lassen sich bis zur Grenzschicht zum AlN hinabverfolgen. Am Punkt D lässt sich eine Überlagerung des GaN-Gitters mit dem Gitter der Ausscheidung (Durchmesser etwa 10 $\mathrm{nm}$ ) in Form eines Moiré-Effektes erkennen. Bei E ziehen sich Stapelfehler durch die Schicht bis zur Ausscheidung bei D, die sich oberhalb und unterhalb eines Bereiches von hoher MnKonzentration befinden. 


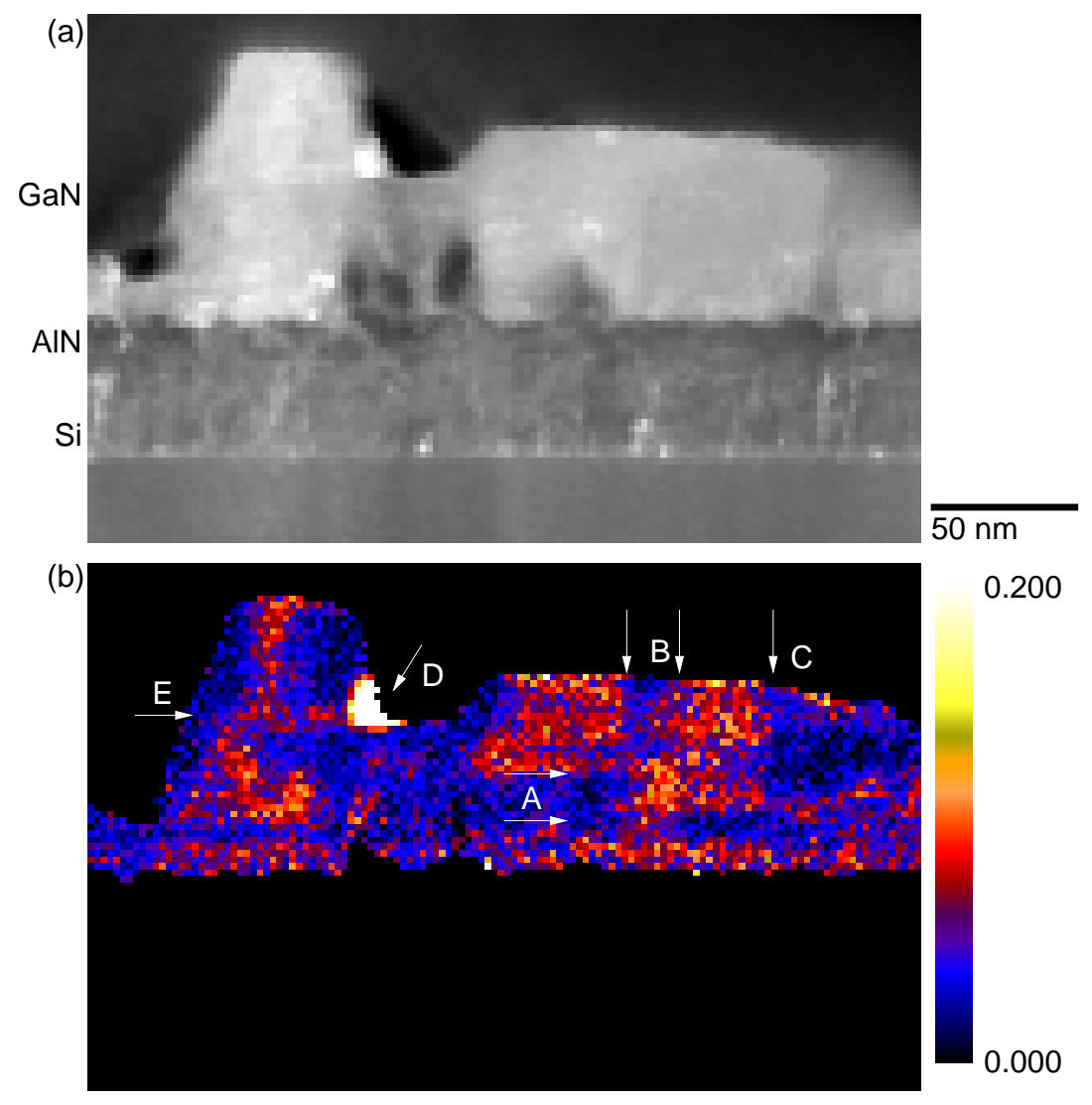

Abbildung 6.15: (a) STEM-Aufnahme. (b) EDX-Messung: das Verhältnis der Zählraten der Mn-K $\alpha$-Linie zur Summe der Mn-K $\alpha$ und Ga-K $\alpha$-Linien ist aufgetragen. Die Summe der beiden Zählraten ist proportional zur Probendicke, so dass die Abbildung nur chemische Unterschiede zeigt. Zur besseren Sichtbarkeit wurden die Punkte mit weniger als 50 Ga-Zählereignissen schwarz gefärbt. Die Buchstaben bezeichnen die Bereiche, die auch in Abb. 6.16 markiert sind. 

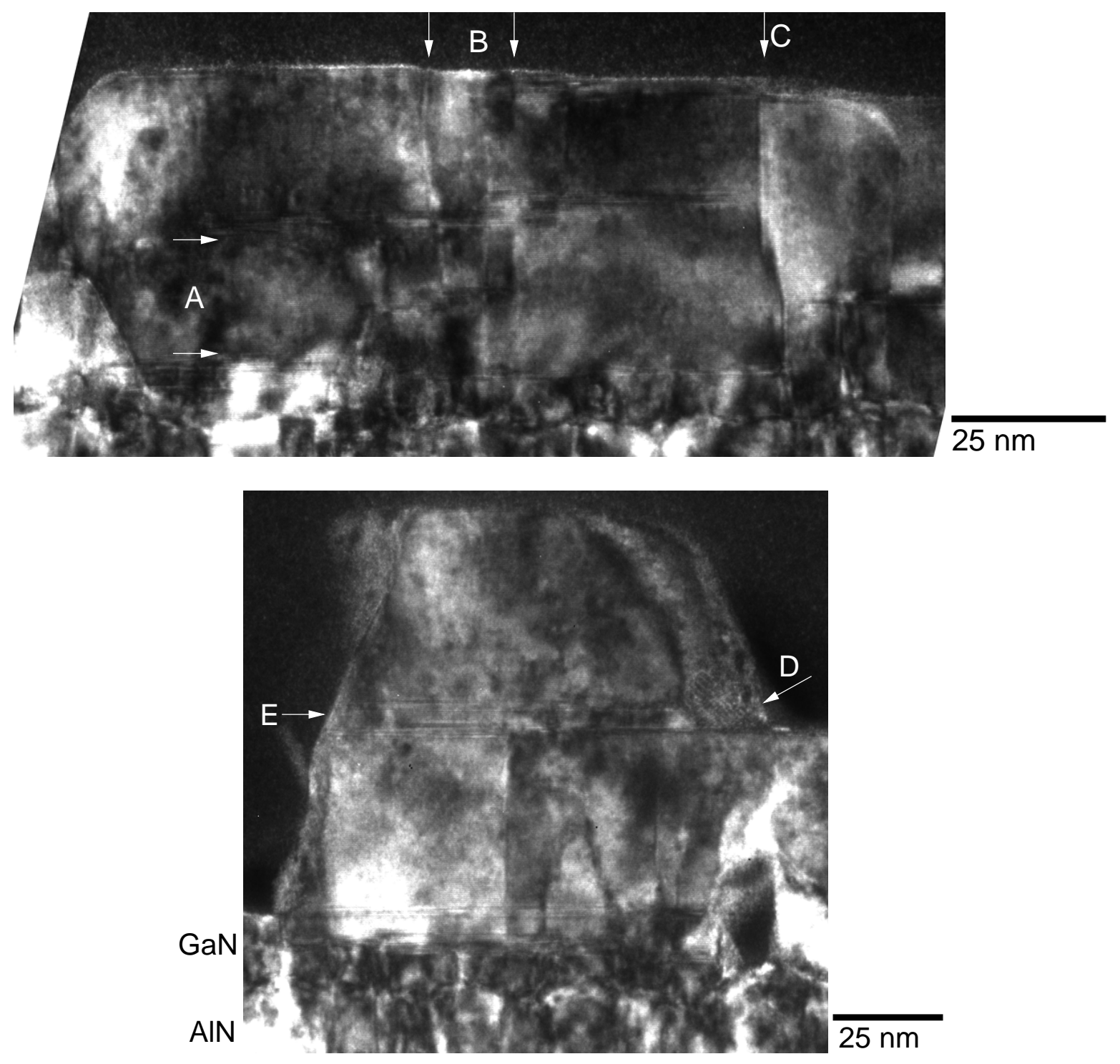

Abbildung 6.16: Dunkelfeldaufnahmen mit $g=(0002)$ aus dem gleichen Probenbereich, wie in Abb. 6.15. Die mit Buchstaben markierten Bereiche entsprechen denen in Abb. 6.15. Der Bereich A ist nach oben und unten durch Stapelfehler begrenzt, die Bereiche B und C zeigen vertikal verlaufende Defekte. Bei D ist eine Ausscheidung zu erkennen, bei E befinden sich wieder Stapelfehler. 
In der HRTEM-Abbildung (Abb. 6.17) des Defektes bei C lässt sich eine starke Musteränderung in der Mitte der Abbildung auf einem etwa $3 \mathrm{~nm}$ breiten Bereich erkennen, die Änderung am linken Rand dieses Bereiches ist sehr deutlich. Eine exakte Identifizierung dieses Defektes ist nicht möglich, da Auswertungen wie in Kapitel 5 nur bei dünnen Probenstellen möglich sind, hingegen ist eine dicke Probenstelle notwendig, um die für die hier gezeigten EDX-Messungen notwendigen Zählraten zu erreichen.

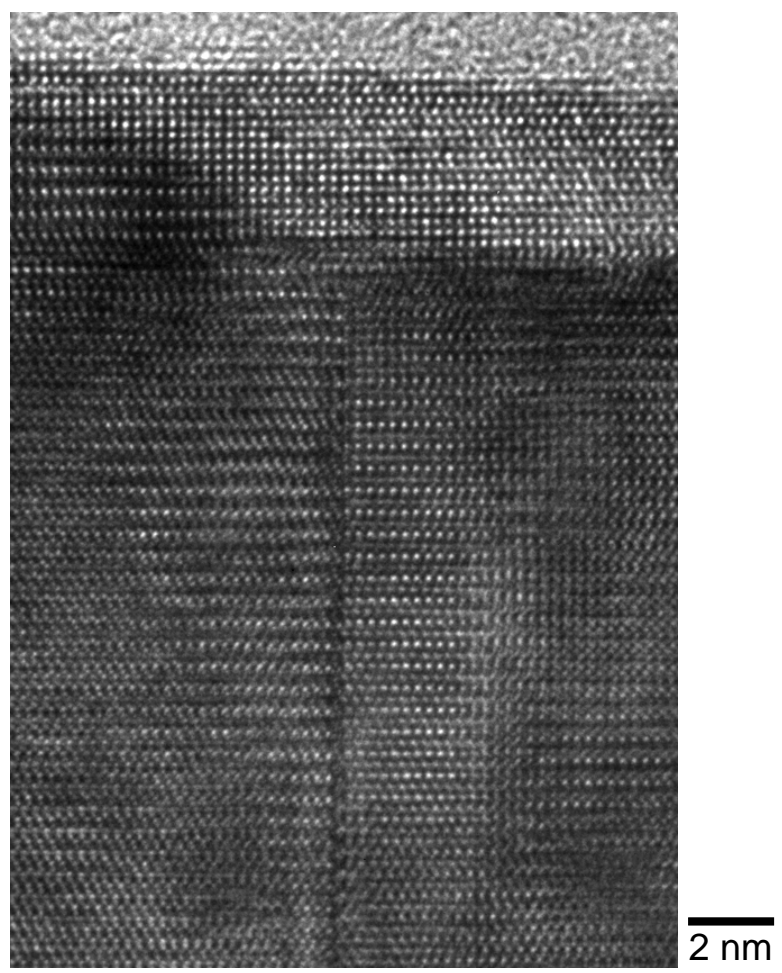

Abbildung 6.17: HRTEM-Abbildung ([11 $\overline{2} 0]$-Zonenachse) des Defektes bei Markierung C in Abb. 6.15 bzw. 6.16 .

In Abb. 6.18 ist eine EDX-Messung einer weiteren Probenstelle gezeigt. Die Punkte mit weniger als 30 Ga-Zählereignissen sind wieder schwarz gefärbt. Insbesondere in der Mn-reichen Schicht zwischen dem oberen inselartigen Bereich und der unteren geschlossenen GaN-Schicht lassen sich wieder basale Defekte finden. Strukturelle Defekte, die den Mn-reichen Bereich innerhalb der Insel vertikal begrenzen, sind nicht zu finden. Um die ungleichmäßige Mn-Verteilung zu quantifizieren, wurden an den markierten Orten zusätzlich Punktmessungen durchgeführt:

\begin{tabular}{cccccc} 
& $\mathrm{A}$ & $\mathrm{B}$ & $\mathrm{C}$ & $\mathrm{D}$ & $\mathrm{E}$ \\
\hline \hline $\mathrm{Mn}$ & 0.23 & 0.08 & 7.37 & 3.92 & 1.27 \\
$\mathrm{Ga}$ & 99.77 & 99.92 & 92.63 & 96.08 & 98.73 \\
\hline
\end{tabular}

Die mittlere Mn-Konzentration dieser Schicht lag bei 2.6 at\%.

Die in den hier untersuchten Proben beobachtete inhomogene Verteilung der gelösten MnAtome in der GaN-Schicht wurde bisher noch nicht in der Literatur berichtet. Auch wurden 


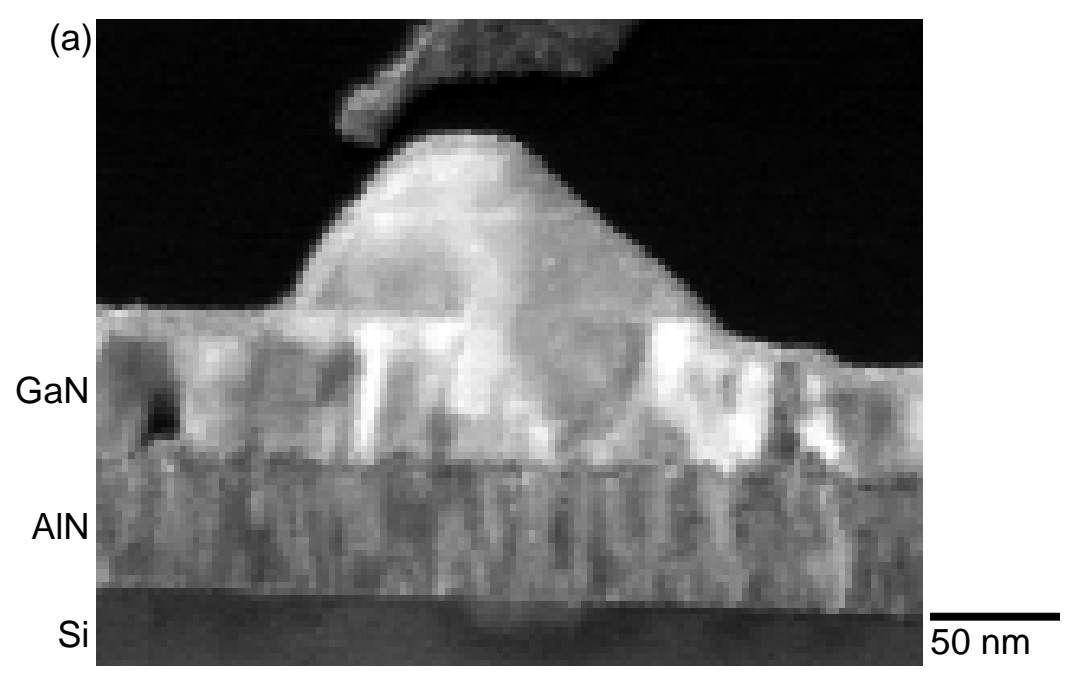

(b)

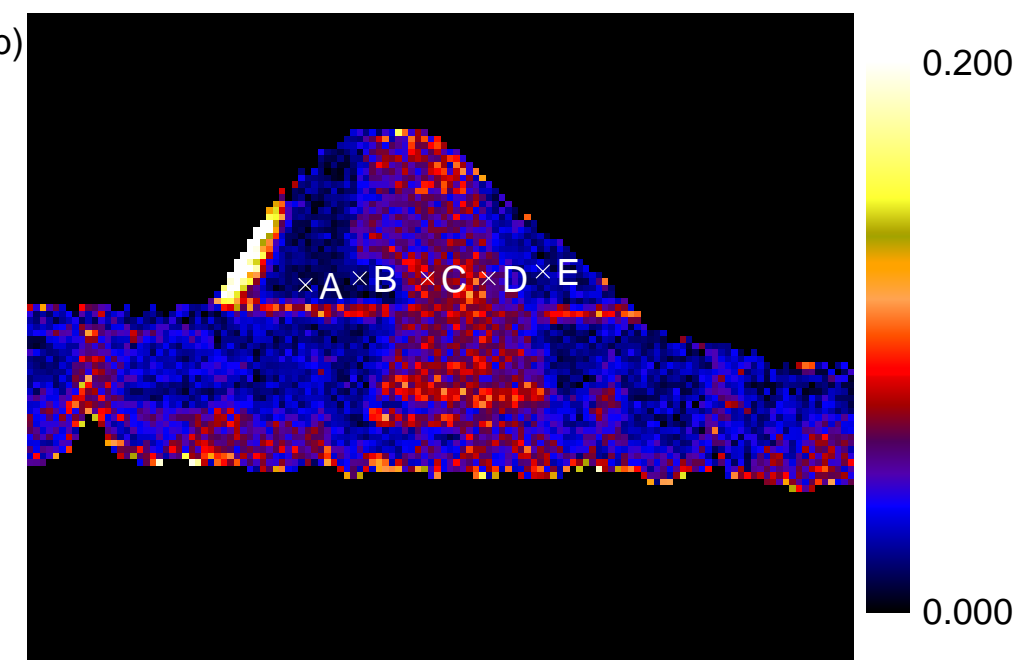

Abbildung 6.18: (a) STEM-Aufnahme. (b) EDX-Messung: das Verhältnis der Zählraten der Mn-K $\alpha$-Linie zur Summe der Mn-K $\alpha$ und Ga-K $\alpha$-Linien ist aufgetragen. Zur besseren Sichtbarkeit wurden die Punkte mit weniger als $30 \mathrm{Ga}$-Zählereignissen schwarz gefärbt. Die Buchstaben bezeichnen die Orte der Punktmessungen (s. Text). 
von keinem Autor bisher die GaN:Mn-Schichten auf eine homogene Verteilung hin untersucht. In Kapitel 8 wird die hier gefundene Mn-Verteilung ausführlicher diskutiert. 


\section{Gitterplatzbestimmung}

Wie in den folgenden Abschnitten erläutert wird, lässt sich die anomale Absorption schneller Elektronen in Kristallen, die aufgrund von Channeling-Effekten auftritt, nutzen, um zu untersuchen, zu welchen Anteilen die Mn-Atome im GaN-Wirtsgitter substitutionell eingebaut werden. Der Name der verwendeten Technik, "Atom Localization by Channeling Enhanced Microanalysis", kurz ALCHEMI, wurde von Spence und Taftø eingeführt [128], die erstmals diesen Effekt zur Lokalisierung von Fremdatomen benutzten.

Im folgenden Abschnitt wird das Grundprinzip der ALCHEMI-Messung erläutert. Der Abschnitt 7.2 erklärt, wie die Technik konkret zur Lokalisierung der Mn-Atome in der Einheitszelle angewendet wird. In Abschnitt 7.3 werden die experimentellen Bedingungen für die Aufnahme der Profile besprochen. Abschnitt 7.4 behandelt die Simulation der Profile. Das Vorgehen beim Vergleich der experimentellen und simulierten Profile sowie bei der Bestimmung der Gitterplatzbesetzung wird in Abschnitt 7.5 beschrieben.

\subsection{Channeling und anomale Absorption}

Trift der Elektronenstrahl entlang einer Orientierung hoher Symmetrie in den Kristall, wie z.B. entlang einer Zonenachse, so tritt ein Effekt auf, der als Channeling bezeichnet wird: die eingestrahlten Elektronen werden vorwiegend entlang von Atomsäulen geführt, während die Intensität des Elektronenstrahls in den Löchern der Struktur stark verringert ist. In Abb. 7.1a wird eine klassische Erklärung dieses Effektes gegeben. Die Elektronen werden von den positiven Atomrümpfen in den Atomsäulen angezogen. Das führt dazu, dass die Elektronen entlang ihres Weg durch den Kristall um die Atomsäulen pendeln. Durch diese Selbstfokussierung des Elektronenstrahls kommt es zu einer höheren Dichte der Elektronen an den Orten der Atome, was zu einer geringeren Dichte zwischen den Atomsäulen führt. Quantenmechanisch wird dieser Effekt durch die dynamische Beugungstheorie (Vielfachstreuung) beschrieben.

In Abb. 7.1b ist als Beispiel die berechnete Elektronenintensität für Kupfer in [001]-Zonenachsenorientierung gezeigt, sowie die für einen leicht gekippten Elektronenstrahl (die Strahlverkippung $\boldsymbol{K}_{t}$ entspricht dem (100)-Streuvektor). Während für beide Orientierungen das Channeling in Form der entlang der Atomsäulen konzentrierten Elektronendichte zu erkennen ist, fällt auf, dass die Stärke dieses Effektes von der Probenverkippung abhängt: Die Maxima in den Atomsäulen weisen im verkippten Fall geringere Intensität auf. Deutlicher wird dies, wenn die Elektronenintensität entlang des Strahls aufintegriert wird (s. Abb. 7.2). Die integrierte Intensität am Ort der Atomsäulen nimmt in diesem Beispiel um etwa $40 \%$ ab.

Im Beispiel Abb. 7.1b ist noch ein weiterer Effekt zu beobachten. Die Stärke der Intensitätsmaxima an den Orten der Atomsäulen, bzw. das Channeling, nimmt mit zunehmender Probendicke ab. Das Channeling ist ein Effekt der kohärenten Vielfachstreuung. Inkohärente 
(a) Einfallender Elektronenstrahl

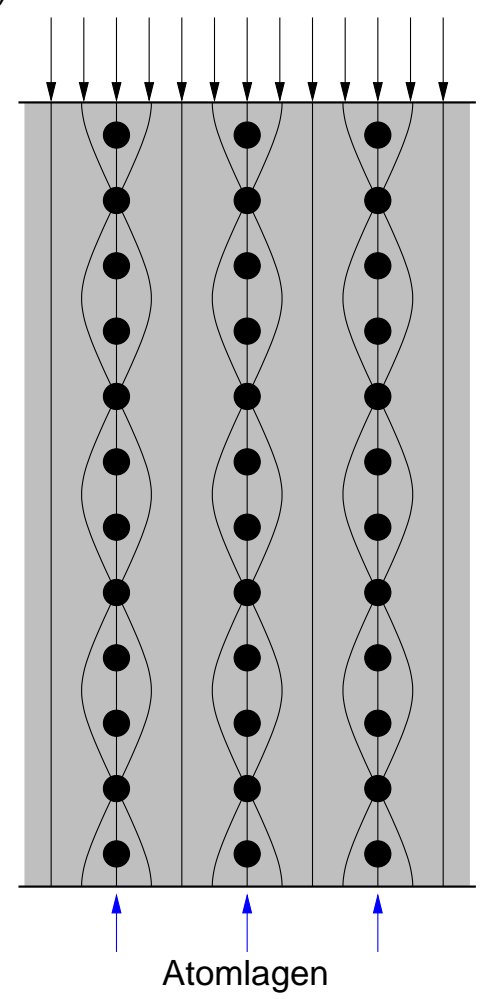

(b) Elektronenintensität

$\mathrm{Cu}[001]$

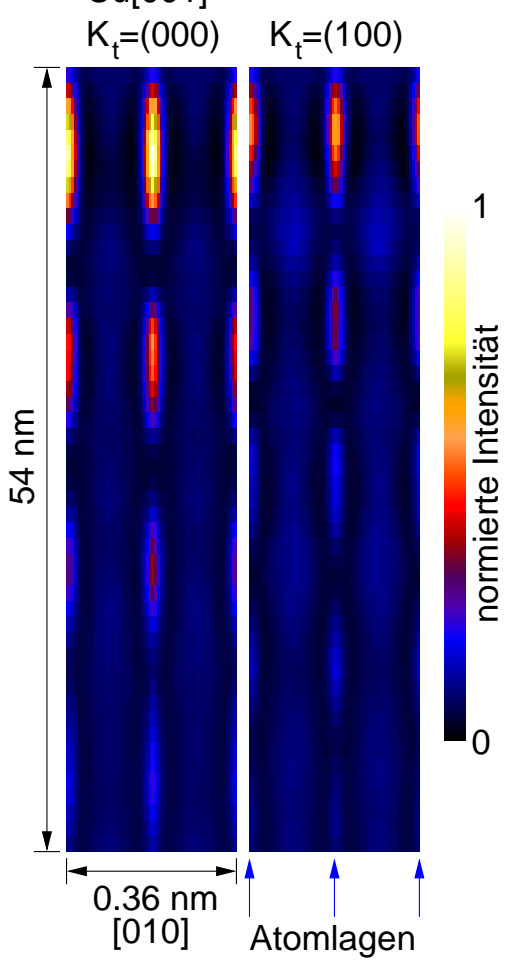

Abbildung 7.1: (a) Klassische Erklärung des Channelings: die Elektronen werden von den positiv geladenen Atomrümpfen (hier durch Punkte symbolisiert) angezogen, wodurch sie entlang ihres Weges um die Atomsäulen pendeln. (b) Berechnete Elektronendichte für [001]-Kupfer ohne und mit Strahlverkippung entlang des Elektronenstrahls (Beschleunigungsspannung $200 \mathrm{kV}$ ). Das obere Ende ist die Probenvorderseite, das untere Ende die Rückseite. Die Horizontale ist die [010]-Richtung.

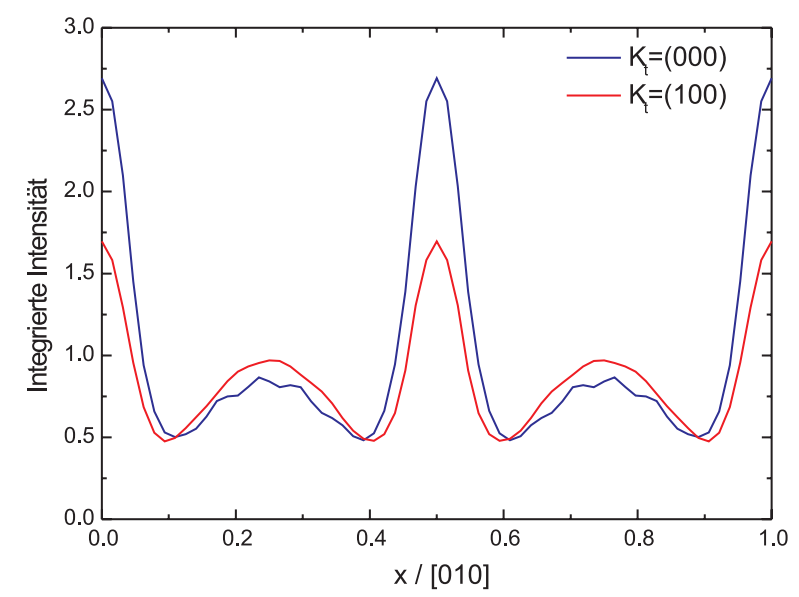

Abbildung 7.2: Entlang des Elektronenstrahls über $54 \mathrm{~nm}$ Probendicke integrierte Intensität für Kupfer (s. Abb. 7.1b) in [010]-Richtung. Die Atomsäulen befinden sich bei den Relativkoordinaten 0.0, 0.5 und 1.0. 
Streuprozesse in der Probe (vorwiegend durch thermisch diffuse Streuung) zerstören die Kohärenz der Elektronenwelle und verringern somit das Channeling. Die durch solche Prozesse gestreuten Elektronen tragen nur noch zu einem diffusen Hintergrund bei [129]. Bei hinreichend dicken Kristallen ergibt sich daher nur noch eine homogene Elektronendichte.

Zusammengefasst führt das Channeling dazu, dass die Elektronendichte innerhalb der Einheitszelle nicht gleichmäßig verteilt ist. Die Größe dieses Effektes und auch die Orte der Einheitszelle, auf denen die Elektronen lokalisiert sind, hängen dabei stark von der Einstrahlrichtung in den Kristall ab.

Streuprozesse, wie z.B. TDS oder die Ionisierung der inneren Atomschalen, sind an den Orten der Atome lokalisiert. Da die Dichte der Elektronen an den Orten der Atome durch das Channeling von der Einstrahlrichtung abhängig ist, wird auch die Anzahl dieser Streuprozesse richtungsabhängig. Diese Richtungsabhängigkeit der Absorption wird als anomale Absorption bezeichnet, im Gegensatz zur ,normalen“ Absorption, die richtungsunabhängig ist. Wird Röntgenstrahlung als Primärstrahlung benutzt, ist dieser Effekt auch als Borrmann-Effekt bekannt.

\subsubsection{Grundidee von ALCHEMI}

Die Ionisierung der inneren Atomschalen $(\mathrm{K}, \mathrm{L}, \ldots)$ durch die schnellen Elektronen kann zur Emission von charakteristischer Röntgenstrahlung führen. Die Intensität der charakteristischen Röntgenstrahlung für die verschiedenen Elemente wird bei einer EDX-Messung quantitativ untersucht.

Zunächst sei angenommen, dass die dafür nötigen Streuprozesse, d.h. die Ionisierung der Atome, genau am Ort der Atome stattfinden. Diese Annahme ist zumindest für die K-Linien schwerer Elemente hinreichend erfüllt (s. Abschnitt 7.1.2). Durch das Einstellen verschiedener Einstrahlrichtungen des Elektronenstrahls lässt sich aufgrund des Channelings die Elektronendichte an bestimmten Orten der Einheitszelle variieren. Befinden sich an diesen Orten Atome einer bestimmten Sorte, so wird auch die Intensität der zugehörigen charakteristischen Röntgenstrahlung variieren. Dies ist die Grundidee der ALCHEMI-Technik.

Die ursprüngliche Formulierung [128] kann benutzt werden, um geringe Mengen von Fremdatomen in geordneten Kristallen zu lokalisieren. Gering heißt in diesem Fall, dass die dynamischen Beugungsbedingungen des Wirtskristalls nicht durch die Fremdatome beeinflusst werden. In einem geordneten Kristall besetzen z.B. die Atomsorten A und B zwei unterschiedliche Untergitter. Ein Fremdatom der Sorte X kann lokalisiert werden, indem die Röntgenintensitäten der Sorten A, B und X für zwei verschiedene Kristallorientierungen gemessen werden. Die eine Orientierung ist so zu wählen, dass das Channeling zu einer Lokalisierung der Elektronendichte auf vorwiegend dem einen Untergitter führt, was z. B. bei der Anregung eines chemisch sensitiven Reflexes zu erwarten ist. Ein chemisch sensitiver Reflex oder auch Überstrukturreflex ist ein Reflex, der im ungeordneten Kristall verschwinden würde. Die andere Orientierung würde so gewählt werden, dass ein möglichst geringes Channeling auftritt. Durch das Berechnen der Verhältnisse der Röntgenintensitäten für die verschiedenen Elemente und Orientierungen kann nun die Konzentration der Sorte X auf dem A- und auf dem B- Untergitter bestimmt werden.

Die ALCHEMI-Technik wurde von verschiedenen Autoren erweitert, um z.B. auch die Anwendung auf weniger geordnete Kristalle, auf Wirtskristalle mit vielen Komponenten oder auf 
größere Konzentrationen der Fremdatome zu ermöglichen. Eine Übersicht der verschiedenen ALCHEMI-Formulierungen ist bei Jones zu finden [130]. Der Grundzug dieser Methoden ist es, die Variation der Röntgensignale der Komponenten des Wirtskristalls mit der Kristallorientierung als Referenz zu verwenden, um die Variation des Röntgensignals der Fremdatomspezies einem Gitterplatz zuzuordnen.

ALCHEMI lässt sich auch auf eine andere Weise durchführen [131, 132, 133, 134], die ebenfalls in dieser Arbeit verwendet wird. Dazu werden die Röntgensignale der unterschiedlichen Elemente für verschiedene Kippwinkel des Elektronenstrahls um eine Orientierung mit starkem Channeling herum gemessen (Rocking Curve). Die sich so ergebenden Röntgenprofile (auch ,incoherent channeling patterns" genannt) können mit simulierten Profilen für verschiedene Positionen des betrachteten Elements innerhalb der Einheitszelle verglichen werden. Diese Vergleiche können rein qualitativ [131, 132], aber auch quantitativ [133, 134] geschehen. Dieses Vorgehen, das es zudem ermöglicht, interstitielle Gitterplätze zu berücksichtigen, da keine Röntgensignale zur Referenz benötigt werden, wird ausführlicher in Abschnitt 7.2 am konkreten Beispiel von GaN erläutert.

\subsubsection{Delokalisierung}

Im letzten Abschnitt wurde die Annahme gemacht, dass die Streuprozesse, die zur Emission der charakteristischen Röntgenstrahlung führen, am Ort der Atome selbst stattfinden, d.h. dass sich das (inelastische) Ionisierungsstreupotential als Superposition von $\delta$-Funktion an den Orten der entsprechenden Atome modellieren lässt. Diese Annahme ist gerechtfertigt solange die wahre Ausdehnung dieses Potentials klein gegen die Längenskala der aufgrund von Channeling entstehenden Variationen der Elektronendichte ist. Als Delokalisierung werden die Effekte bezeichnet, die auftreten, wenn diese Annahme nicht mehr gerechtfertigt ist.

Ein Effekt, der prinzipiell zur Verschmierung des Streupotentials führt, ist die thermische Vibration der Atome. Diese Verschmierung wird üblicherweise durch den Debye-Waller-Faktor beschrieben. Es hat sich allerdings gezeigt, dass dieser Effekt einen zu vernachlässigenden Einfluss auf den Streuquerschnitt hat [135].

Gravierenderen Einfluss hat jedoch ein anderer Effekt: Die Energie, die ein schnelles Elektron bei der inelastischen Streuung an einem Atom auf dieses überträgt, hängt vom Streuparameter ab, das ist der Abstand zwischen der Bahn des einfallenden Elektrons und dem Atom. Dieser Energieübertrag ist für kleinere Streuparameter größer. Aus dieser Abhängigkeit ergibt sich ein maximaler Streuparameter, bei dem noch genug Energie übertragen wird, um die entsprechende Schale des Atoms zu ionisieren. Somit wird der Streuquerschnitt abhängig von den Ionisierungsenergien der verschiedenen Elemente und wird dabei für kleinere Energien größer. Daher tritt die Delokalisierung vorwiegend für leichte Elemente oder L-Linien auf.

Die Delokalisierung lässt sich bei der Simulation der Röntgenemission berücksichtigen, indem die Form des Streupotentials analog zu den Atomformfaktoren der elastischen Streuung explizit mit einbezogen wird (s. Abschnitt 7.4). Allen [136] hat für Sauerstoff gezeigt, dass bei diesem Vorgehen die simulierten Röntgenprofile hinreichend mit den experimentell gemessenen übereinstimmen. Eine Übersicht und Bewertung von Verfahren, die Delokalisierung für ALCHEMI-Formulierungen zu berücksichtigen, die ohne Simulation der Röntgenprofile auskommen, ist bei Oxley zu finden [137]. 


\subsection{Vorgehensweise bei GaN:Mn}

Mittels ALCHEMI wird in dieser Arbeit untersucht, in welchem Umfang die Mn-Atome substitutionell oder interstitiell in den GaN-Kristall eingebaut werden. Neben dem substitutionellen Ga-Platz werden die interstitielle Oktaeder-Lücke $(\mathrm{O})$ und die interstitielle Tetraeder-Lücke (T) als mögliche Plätze in Betracht gezogen. Da im Wirtskristall die interstitiellen Gitterplätze unbesetzt sind, können keine Röntgensignale als Referenz für diesen Gitterplatz verwendet werden. Daher wird hier die ALCHEMI-Messung durchgeführt, indem die Röntgensignale für verschiedene Einstrahlrichtungen um eine Symmetrieorientierung herum gemessen werden. Das auf diese Weise erhaltene Röntgenprofil (charakteristisches Röntgensignal gegen Einstrahlrichtung) soll mit simulierten Profilen für die verschiedenen möglichen Gitterplätze verglichen werden. Bei der Simulation wird dabei von einem perfekten Kristall ausgegangen, der Einfluss von evtl. vorhandenen Defekten auf die mittleren Beugungseigenschaften des Wirtskristalls wird somit vernachlässigt.

Das Grundprinzip dieser Messung ist in Abb. 7.3 skizziert. Die entlang des Elektronenstrahls integrierte Elektronenintensität ist in Graustufen dargestellt (aus Simulationen, Beschleunigungsspannung $200 \mathrm{kV}$, nur dynamisch gestreuter Anteil, keine diffusen Anteile). Die benutzten Anregungsbedingungen sind eine systematische Reihe von (0002)-Reflexen. Die Strahlverkippung ist über den Vektor $\boldsymbol{K}_{t}$ vom Nullstrahl zum Laue-Zentrum in Koordinaten des reziproken Raumes angegeben. Auf die integrierte Elektronenintensität ist die atomare Struktur der GaNEinheitszelle samt exemplarischen interstitiellen Gitterplätzen projiziert. Es ist zu erkennen, dass die Elektronenintensitäten an den verschiedenen Gitterplätzen mit dem Einstrahlwinkel variieren. Unter der Annahme, dass die emittierte charakteristische Röntgenstrahlung proportional zur Elektronenintensität am Ort der Atome ist, variieren die zu erwartenen Röntgensignale entsprechend. So ist beispielsweise für Atome in der Oktaederlücke bei $\boldsymbol{K}_{t}=[000 x]$ für $-1 \leq x \leq 1$ eine geringe Intensität zu erwarten, für $x= \pm 2$ hingegen eine große. Für den GaPlatz gilt gerade das Gegenteil. Zu beachten ist, dass die Elektronenintensitäten in der oberen wie in der unteren Hälfte der Einheitszelle identisch sind und es unter diesen (und auch den anderen in dieser Arbeit verwendeten) Anregungsbedingungen nicht möglich ist zwischen $\mathrm{O}$ und T-Platz zu unterscheiden.

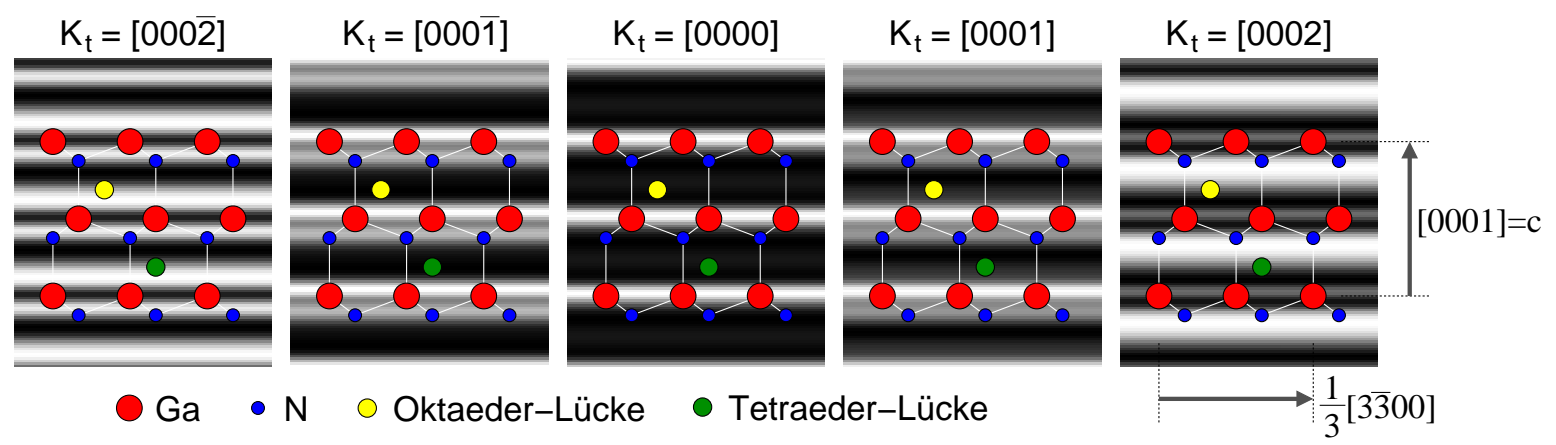

Abbildung 7.3: Grundprinzip der ALCHEMI-Messung zur Lokalisierung der Mn-Atome. In Graustufen ist die entlang des Elektronenstrahls integrierte Elektronendichte dargestellt, auf die die atomare Struktur der GaN-Einheitszelle samt interstitiellen Plätzen (exemplarisch) projiziert ist.

Da der Mn-Gehalt der untersuchten Proben im Bereich von 1-4 at\% (bezogen auf die Gesamt- 
metallkonzentration) liegt, ist nicht zu erwarten, dass die dynamischen Beugungsbedingungen von den Mn-Atomen abhängen.

\subsection{Experimentelles Vorgehen}

Als Anregungsbedingungen für die Röntgenmessungen wurde die systematische Reihe von (0002)-Reflexen sowie von (11 $\overline{2} 0)$-Reflexen gewählt. Von einer systematischen Reihe (oder auch von planaren Channeling-Bedingungen) wird gesprochen, wenn nur ganzzahlige Vielfache des der Reihe zugrundeliegenden Reflexes angeregt werden. Diese Bedingungen haben den Vorteil, dass das im Kristall entstehende Wellenfeld entlang der Richtung senkrecht zur systematischen Reihe und zum Elektronenstrahl konstant ist. Somit haben auch geringe Strahlverkippungen senkrecht zur Reihe keinen Einfluss auf die Messung, und es müssen nur die Verkippungen entlang der Reihe kontrolliert werden. Auch ist der Einfluss der Delokalisierung auf das Experiment geringer als in einer Zonenachsenorientierung [130, 133].

Zunächst wurden die Symmetrieorientierung und die systematische Reihe mittels des Goniometers eingestellt. Die Variation der Verkippung um diese Orientierung herum wurde durch Kippen des Elektronenstrahls im Mikroskop erreicht. Den Strahl zu kippen, hat gegenüber dem Verkippen des Kristalls mittels Goniometer mehrere Vorteile: Zum einen lässt sich der Kippwinkel besser kontrollieren und messen (s. Anhang A). Zum anderen bleibt der Winkel zwischen EDX-Detektor und Probe konstant. Bei einer Kippung mittels Goniometer würde sich die Probe in alle drei Raumrichtungen verschieben, was eine Nachführung der Probe notwendig macht. Die leichte Verschiebung des Elektronenstrahls aufgrund der Linsenfehler (s. Abschnitt 3.4.2) kann durch eine Strahlverschiebung im Mikroskop ausgeglichen werden. Ein Nachteil der Strahlverkippung ist, dass sich aufgrund der Linsenfehler auch die Form des Leuchtfleckes (s.u.) verändert. Dadurch ist es nicht möglich, große Strahlverkippungen zu realisieren.

Die Röntgenspektren wurden für einen Kippwinkelbereich von $\pm 0.8^{\circ}$ in Schritten von typischerweise $0.13^{\circ}$ entlang der systematischen Reihe gemessen. Vor der Messung wurde der Leuchtfleck immer wieder auf dieselbe Probenstelle zentriert und eine Aufnahme von der Probe im Leuchtfleck gemacht. Die Messzeit für die einzelnen Röntgenspektren betrug $50 \mathrm{~s}$ „LiveTime“, das ist die Zeit bei der Röntgenstrahlung tatsächlich detektiert wird. Nachdem ein Röntgenstrahl den Detektor getroffen hat, wird immer eine kleine Zeit benötigt (die „DeadTime“), um das Signal zu analysieren. Die eigentliche Messzeit setzt sich aus beiden Anteil zusammen. Gemessen wurde mit einem Oxford-Link-ISIS System und einem Si:Li EDX Detektor. Bei der Messung wurden die Intensitäten der Ga-K und Mn-K Linien bestimmt (bzw. für die Messung in Abschnitt 7.6 die Al-K-Linie).

Von der Verwendung der N-Linie wurde aus mehreren Gründen abgesehen: Für diese Linie ist ein schlechtes Signal-Rausch-Verhältnis zu erwarten, da im niedrigen Energiebereich $(<1$ $\mathrm{keV}$ ) die Detektoreffizienz gering ist, während der Hintergrund, d.h. die Bremsstrahlung, hier große Intensitäten aufweist. Zusätzlich überlappt die N-Linie mit der O-Linie und der C-Linie. Gerade das Signal des Kohlenstoffs ist aufgrund von Probenkontamination unvermeidlich. Außerdem sind in diesem Energiebereich weitere Röntgensignale aufgrund der Tatsache zu erwarten, dass die untersuchten Probenstellen sich am Rand der Probe befanden, wodurch auch Röntgensignale aus der Klebeschicht (im Wesentlichen leichte Elemente, wie C und O) zum 
Spektrum beitragen. Auch müsste die Absorption der N-Röntgenlinien innerhalb der Probe mit berücksichtigt werden. Diese ist für die schweren Elemente hingegen zu vernachlässigen.

\section{Fokussierung des Elektronenstrahls}

Es hat sich gezeigt, dass für das Gelingen der ALCHEMI-Messung eine sorgfältige Kontrolle der Beleuchtung (Strahlengang in Abb. 7.4) notwendig ist. Da die späteren Messungen in einem kleinen Probenbereich (Durchmesser $75 \mathrm{~nm}$ ) durchgeführt werden, sind sie sehr sensitiv auf die Beleuchtung.

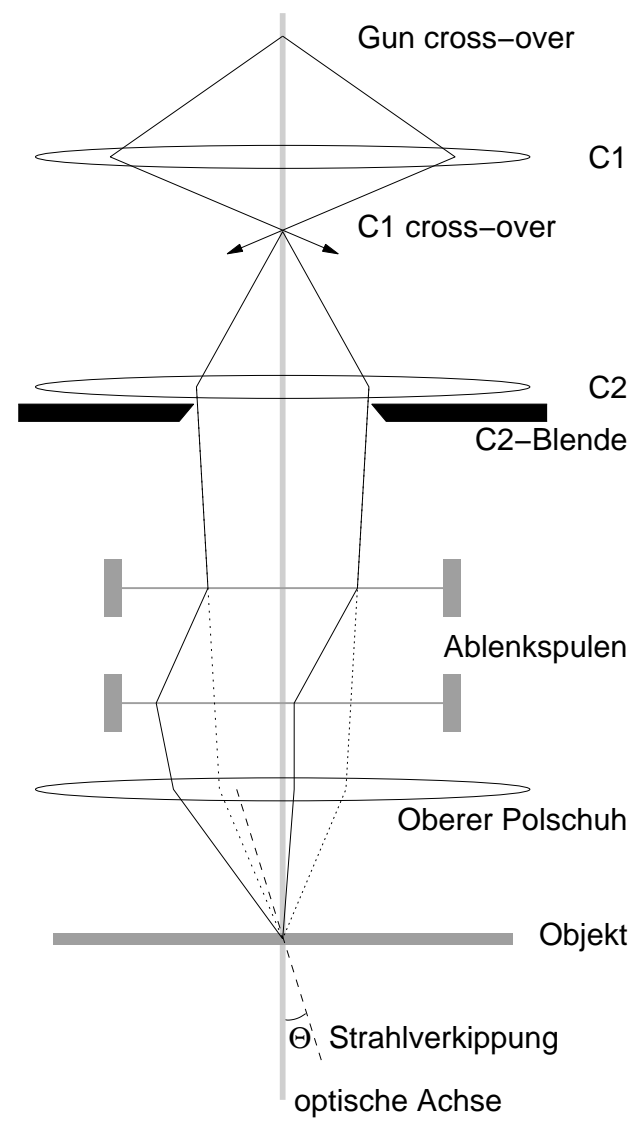

Abbildung 7.4: Schematischer Strahlengang des Beleuchtungssystems. Der Winkel der Strahlverkippung ist $\Theta=\lambda K_{t}$, mit $\lambda$ als der Wellenlänge der Elektronen.

Sämtliche Messungen wurden im Abbildungsmodus des Mikroskops durchgeführt. Vor den Messungen wurde die Intensitätsverteilung im Leuchtfleck sorgfältig justiert und diesem mit den Stigmatoren des Beleuchtungssystems eine möglichst symmetrische Form (Kreisform) gegeben. Mit Hilfe der CCD-Kamera wurde kontrolliert, dass sowohl die Intensitätsverteilung im Leuchtfleck mittig, als auch die Intensitäten im Beugungsmaximum am Rand des Leuchtfleckes gleichmäßig waren. Die ALCHEMI-Messungen wurden mit der $50 \mu \mathrm{m}$ C2-Blende und den C1-Einstellungen (Spotsize) 5 oder 6 durchgeführt. Nach der Fokussierung der Probe mittels der Objektivlinse (dies ändert auch die Erregung des oberen Polschuhs) wurde der 
Strahl durch Verändern der C2-Linsenerregung auf einen Durchmesser von $75 \mathrm{~nm}$ fokussiert. Nach dieser Fokussierung wurden die Einstellungen der Linsen nicht mehr geändert. Einzig die Strahlverkippung und Strahlverschiebung durch die Ablenkspulen wurden während der Messung geändert.

Um zu kontrollieren, dass sich die Intensität des Elektronenstrahl während der Messung nicht verändert, wurden Aufnahmen des Leuchtflecks vor und nach der Messung, sowie für die extremen Strahlverkippungen $\left( \pm 0.8^{\circ}\right)$ gemacht (s. Abb. 7.5). Bei der Strahlverkippung ist zu beachten, dass der Strahl nun nicht mehr parallel zur optischen Achse durch den oberen Polschuh der Objektivlinse läuft, sondern unter einem Winkel $\Theta=\lambda K_{t}$. Dies führt dazu, dass aufgrund der Linsenfehler der Leuchtfleck zum einen leicht größer wird, zum anderen eine leicht elliptische Form annimmt. Da die Gesamtintensität des Strahles konstant bleibt (Änderungen $<1 \%$ ), muss die Intensität im Leuchtfleck daher kleiner werden, was gut in den Abbildungen des Flecks für die extremen Strahlverkippungen zu erkennen ist.
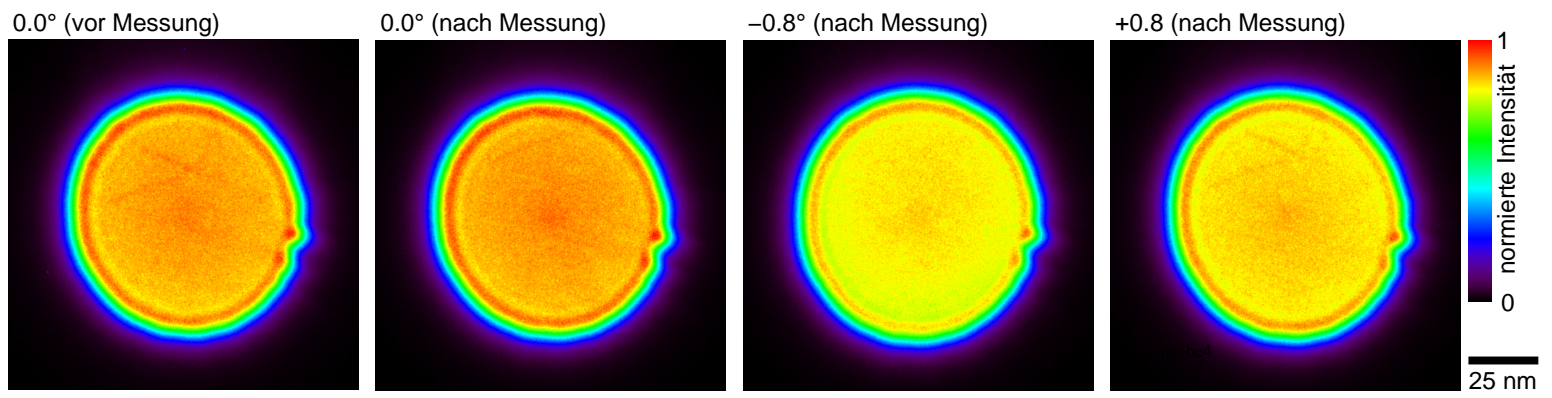

Abbildung 7.5: Intensitätsverteilung im Elektronenstrahl vor und nach der Messung für verschiedene Strahlverkippungen. Die Störung am rechten Rand stammt von einem Fehler in einer der Blenden des Beleuchtungssystems, die linienartige Struktur im Inneren des Leuchtflecks ist ein Artefakt der CCD-Kamera.

Durch die Fokussierung auf einen kleinen Bereich ist der Elektronenstrahl leicht konvergent geworden (s. Strahlengang in Abb. 7.4). Zur Bestimmung der Größe des Konvergenzwinkels wurden im Beugungsmodus Abbildungen ohne Probe im Strahl unter den gleichen Beleuchtungsbedingungen wie bei den ALCHEMI-Messungen aufgenommen (gleicher C1, C2, und Objektivlinsenstrom). Der Durchmesser des Nullstrahls in diesen Abbildungen entspricht dem Konvergenzwinkel des Strahls. Es ergaben sich Konvergenzwinkel von 0.169 ${ }^{\circ}$ für Spotsize 6 und $0.177^{\circ}$ für Spotsize 5. Diese Konvergenz wurde in den Simulationen der Profile berücksichtigt.

\subsection{Simulationen}

Zur Simulation der Röntgenspektren wurde eine Blochwellenrechnung für nicht-zentrosymmetrische Kristalle mit komplexen Streupotentialen nach der Bethe-Methode (siehe z.B. [82]) implementiert.

Für eine bestimmte Kristallorientierung wird zunächst das aus der dynamischen Vielfachstreuung resultierende Wellenfeld (in Form von Koeffizienten, Wellenvektoren und Anregungen der Blochwellen) für die eingestellten Beugungsbedingungen berechnet. Die Rechnung wird mit typischerweise 21 Strahlen (nullte Laue-Zone) aus der systematischen Reihe um die Symmetrieorientierung herum durchgeführt. Die Parametrisierung der reellen und der imaginären 
Atomformfaktoren wurde von Weickenmeier und Kohl übernommen [83]. Die so berechneten imaginären Formfaktoren berücksichtigen nur TDS als inkohärenten Streuprozess. Da andere Streuprozesse, wie z.B. Plasmonenanregung, im Wesentlichen nur für sehr kleine Streuvektoren Beiträge liefern, wird ein additiver Term für das mittlere Absorptionspotential der Probe $V_{0}^{\prime} \rightarrow V_{0}^{\prime}+\alpha V_{0}$ mit der empirischen Absorptionskonstante $\alpha$ eingeführt, um auch diese Prozesse zu berücksichtigen. Die spätere Anpassung der simulierten an die gemessenen Röntgenintensitäten ergab die besten Übereinstimmungen für $\alpha=0.06$. Bei den Simulationen wurden die Debye-Waller-Faktoren für GaN von Schulz übernommen [14]: $B_{G a}=0.0034 \mathrm{~nm}^{2}$ und $B_{N}=0.0040 \mathrm{~nm}^{2}$. Der Ga-Wert wurde ebenfalls für die anderen Metalle (Al und $\mathrm{Mn}$ ) verwendet.

Aus den so berechneten dynamischen Beugungsbedingungen können die totalen Ionisierungsstreuquerschnitte in Abhängigkeit von der Probendicke $t$ für eine bestimmte Atomsorte an bestimmten Gitterplätzen mit den von Allen und Roussow [135], bzw. von Allen und Josefsson $[129,138]$ hierfür hergeleiteten Ausdrücken berechnet werden. Diese Querschnitte bestehen aus zwei Teilen. Der erste Teil beschreibt die Ionisierung durch Elektronen, die sich noch in der dynamischen Vielfachstreuung befinden, d.h. noch Channeling zeigen. Dabei wird das Ionisierungsstreupotential in der lokalen Näherung [129] benutzt, d.h. dieses Streupotential wird wie das elastische Streupotential als Superposition der Streupotentiale der freien Atome, mit den zu untersuchenden Gitterplätzen als Aufpunkten berechnet. Die thermischen Vibrationen der Atome werden auch hier in Form von Debye-Waller-Faktoren berücksichtigt. Der zweite Term im Streuquerschnitt beschreibt die Ionisierung durch Elektronen, die bereits diffus gestreut wurden. Dabei wird diesen eine über die Einheitszelle konstante Intensität zugeordnet, entsprechend wirkt auf sie das mittlere Ionisierungsstreupotential der untersuchten Atomspezies. Die Intensität der diffus gestreuten Elektronen wird dabei aufgrund der Erhaltung der Elektronenzahl als die nicht mehr in der dynamischen Vielfachstreuung enthaltene Elektronenintensität beschrieben. Für die atomaren inelastischen Streufaktoren wurden die in [139] angegebenen Parametrisierungen benutzt, so dass Delokalisierungseffekte möglichst exakt berücksichtigt werden.

Die Röntgenprofile werden simuliert, indem die oben skizzierte Rechnung für jede Strahlverkippung um die Symmetrieorientierung herum durchgeführt wird. Die so berechneten Profile sind proportional zu den messbaren Röntgenprofilen. Der Proportionalitätsfaktor ist dabei linienspezifisch, da nur die jeweiligen Ionisierungsquerschnitte berechnet wurden, in die Intensitäten der Röntgenlinien allerdings noch die Röntgenfluoreszens der Linie und weitere EDX-systemspezifische Parameter (energieabhängige Detektorsensitivität usw.) einfließen. Dieser Faktor muss beim Vergleich des simulierten mit dem berechneten Profil mit angepasst werden. Um den konvergenten Elektronenstrahl zu berücksichtigen, werden die erhaltenen Ionisierungsquerschnitte für jede Einstrahlrichtung über den entsprechenden Konvergenzwinkel integriert.

\subsection{Anpassung und Vergleich der Profile}

Für den Vergleich der gemessenen mit den simulierten Profilen müssen zunächst die Parameter der Simulation an das Experiment angepasst werden. Diese Parameter sind die Dicke der Probe sowie die genaue Strahlverkippung (im Folgenden absolute Verkippung genannt) an den einzelnen Punkten des Profils. Ist die genaue Verkippung für einen Punkt des Profils 
bekannt, können die Verkippungen für die anderen aus der nominellen Strahlkippung des Mikroskops berechnet werden (s. Anhang A). Da es nicht möglich war, die Dicke und die absolute Verkippung während des Experimentes hinreichend genau aus der konvergenten Elektronenbeugung (CBED) oder anderen Techniken zu bestimmen, wurden diese Parameter angepasst: Dazu wurde das simulierte substitutionelle Ga-Profil unter Variation der Probendicke und der absoluten Verkippung bei gleichzeitiger Anpassung eines Proportionalitätsfaktors an das experimentell gemessene Ga-Profil angepasst.

Die gemessenen Röntgenintensitäten sollten possionverteilt sein, da es sich hierbei um ein Zählexperiment handelt. Da die Anzahl der Zählereignisse hinreichend groß ist ( $\gg 20$ ), kann die Verteilung durch die Gaußverteilung genähert werden. Aus diesem Grund wurde für diese und auch für die weiteren Anpassungen eine $\chi^{2}$-Minimierung benutzt. Sämtliche Anpassungen wurden mit der Downhill-Simplex-Methode vorgenommen [107].

Um die Besetzung der Gitterplätze zu bestimmen, d.h. die Frage zu beantworten, zu welchen Anteilen ein Fremdatom substitionell oder interstitiell eingebaut wird, wurde ein etwas anderes Vorgehen gewählt. Es wurde das Verhältnis des Signals des Fremdatoms zum (substitutionellem) Ga-Signal angepasst. Die Signalverhältnisse haben den Vorteil, dass die meisten der weiteren Einflüsse auf das Experiment nur in die Signalintensitäten, nicht aber in ihre Verhältnis eingehen. Daher wird auch die quantitative EDX-Analyse mit den Verhältnissen der Intensitäten durchgeführt [57] (s. auch Abschnitt 2.2.2).

Es wird eine Linearkombination aus den simulierten Röntgenintensitätsverhältnissen für das Fremdatom auf dem interstitiellen sowie auf dem substitutionellen Gitterplatz an das experimentelle Verhältnis angepasst. Die bei der Anpassung für ein Fremdatom A minimierte Größe ist

$$
\chi^{2}=\sum_{x} \frac{\left[\frac{I_{\mathrm{A}, \exp }(x)}{I_{\mathrm{Ga}, \exp }(x)}-\lambda_{\mathrm{s}} \frac{I_{\mathrm{A}, \text { subst }}(x)}{I_{\mathrm{Ga}, \text { subst }}(x)}-\lambda_{\mathrm{i}} \frac{I_{\mathrm{A}, \text { interst }}(x)}{I_{\mathrm{Ga}, \text { subst }}(x)}\right]^{2}}{\frac{1}{I_{\mathrm{Ga}}^{2}(x)} \sigma_{\mathrm{A}}^{2}(x)+\frac{I_{\mathrm{A}, \exp }^{2}(x)}{I_{\mathrm{Ga}, \exp }(x)} \sigma_{\mathrm{Ga}}^{2}(x)},
$$

dabei sind $I_{\mathrm{X}, \exp }(x)$ und $\sigma_{\mathrm{X}, \exp }(x)$ die gemessenen Intensitäten und ihre Standardabweichungen, $I_{\mathrm{X} \text {,subst }}(x)$ und $I_{\mathrm{X} \text {,interst }}(x)$ die simulierten Intensitäten für die jeweiligen Gitterplätze, wobei X die entsprechende Atomsorte darstellt und $x$ über die Verkippungen des experimentellen Profils läuft. Der Nenner berechnet den Fehler des Verhältnisses mit Gaußscher Fehlerfortpflanzung. Das Verhältnis der Koeffizienten $\lambda_{\mathrm{s}} / \lambda_{\mathrm{i}}$ ist das Verhältnis von substitutionellem zu interstitiellem Einbau des Fremdatoms, die Proportionalitätsfaktoren zwischen Experiment und Simulation kürzen sich dabei heraus.

Um eine Standardabweichung für die Gitterplatzbesetzung zu erhalten, wurde eine MonteCarlo-Simulation mit 10000 Punkten verwendet [107]. Das Vorgehen ist dabei analog zu dem in Abschnitt 4.3.3 beschriebenen.

\subsection{Testmessung an $\mathrm{Al}_{0.23} \mathrm{Ga}_{0.77} \mathrm{~N}$}

Zur Überprüfung der hier vorgestellten ALCHEMI-Technik wurde diese auf eine $\mathrm{Al}_{0.23} \mathrm{Ga}_{0.77} \mathrm{~N}$ Schicht angewendet, die in Abb. 7.6 gezeigt ist. Dabei wurde eine systematische Reihe von (0002)-Reflexen als Anregungsbedingung gewählt. Diese Schicht war Teil einer Heterostruktur, die unter optimierten Bedingungen gewachsen wurde (Probe ED162 aus [140]). Bei dieser 
Schicht kann davon ausgegangen werden, dass sämtliches Al substitionell auf den Ga-Plätzen eingebaut wurde. Bei der Messung wurde ein Durchmesser des Leuchtflecks von $\approx 75 \mathrm{~nm}$ verwendet, was dem Durchmesser der bei GaN:Mn-Proben verwendeten Leuchtflecken entspricht.

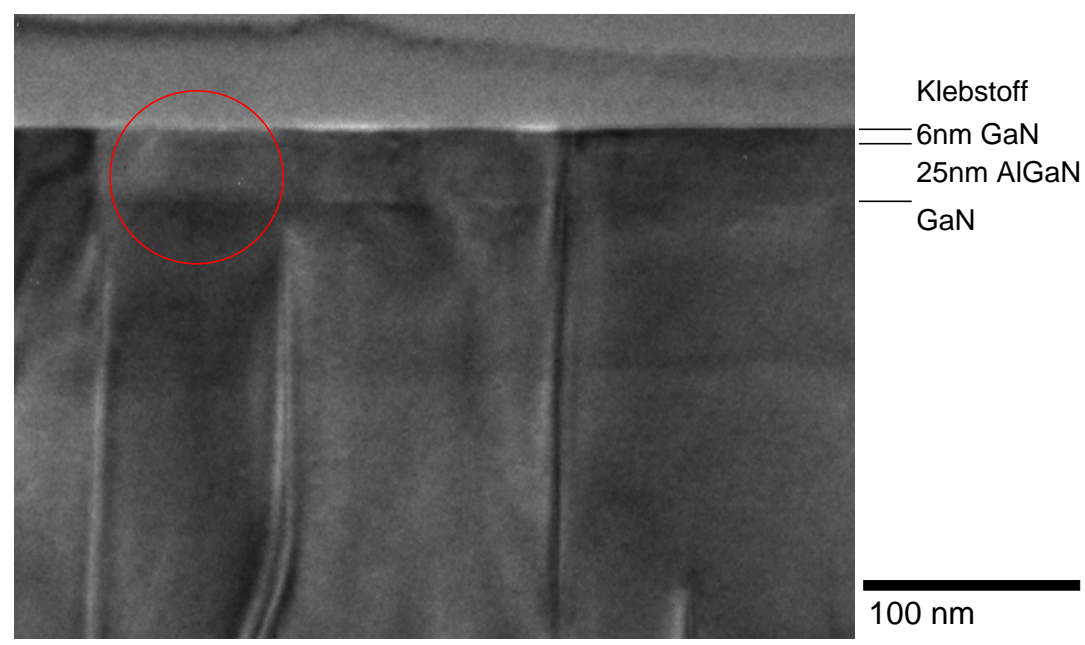

Abbildung 7.6: Hellfeldabbildung der untersuchten AlGaN-Schicht. Der Kreis deutet den untersuchten Bereich und an. Die beiden Versetzungen seitlich des Kreises wurden bei der Messung zur Orientierung benutzt.

Im Gegensatz zur Lokalisierung der Mn-Atome, die hinreichend homogen im Wirtskristall eingebaut werden, muss in dieser Messung berücksichtigt werden, dass der beleuchtete Bereich teilweise aus der zu untersuchenden AlGaN-Schicht und teilweise aus reinem GaN besteht. Die Klebeschicht oberhalb des Kristalls kann ignoriert werden, da sie keinen Beitrag zu den Röntgensignalen für Al und Ga leistet. Da der beleuchtete Bereich während der Strahlverkippung jeweils nachgeführt werden musste, konnte nicht sichergestellt werden, dass sich immer die gleichen Anteile von reinem GaN und der AlGaN-Schicht im beleuchteten Bereich befanden. Um diesen Messfehler zu korrigieren, wurden für jede Strahlverkippung aus den Abbildungen des Leuchtfleckes die beleuchteten GaN- und AlGaN-Flächen $A_{\mathrm{GaN}}$ bzw. $A_{\mathrm{AlGaN}}$ bestimmt und die gemessenen Al-Signale mit dem Faktor $\left(A_{\mathrm{GaN}}+A_{\mathrm{AlGaN}}\right) / A_{\mathrm{AlGaN}}$ korrigiert, so dass das Verhältnis der Ga und Al-Signale einem homogenen AlGaN-Bereich entsprach.

Durch den 23\%-igen Aluminium-Anteil weichen die dynamischen Beugungseigenschaften des Wirtskristalls von denen eines reinen GaN ab, daher wurde als Wirtskristall für die Berechnungen des Wellenfeldes entsprechend $\mathrm{Al}_{0.23} \mathrm{Ga}_{0.77} \mathrm{~N}$ verwendet. Wenn ein größerer Al-Anteil interstitiell eingebaut würde, wäre mit einer weiteren Veränderung der Beugungseigenschaften zu rechnen, was korrekterweise bei der Anpassung durch Neuberechnung der Beugungseigenschaften für verschiedene interstitielle Al-Anteile berücksichtigt werden müsste. Da jedoch Simulationen (s. Abb. 7.7) zeigen, dass die Größe dieses Effektes im Bereich des Fehlers des Al-Signals liegt, wird hier darauf verzichtet. Wird das hier vorgestellte ALCHEMI-Verfahren auf geringe Fremdatom-Konzentrationen angewendet, kann davon ausgegangen werden, dass die Beugungseigenschaften sich nicht ändern.

Abb. 7.8 zeigt die gemessenen Al- und Ga-Profile gemeinsam mit den angepassten Simulationsprofilen. Die Anpassung des Ga-Profils ergab eine Probendicke von $41 \mathrm{~nm}$. Die Bestimmung der 
Substitutionelles Al-Signal

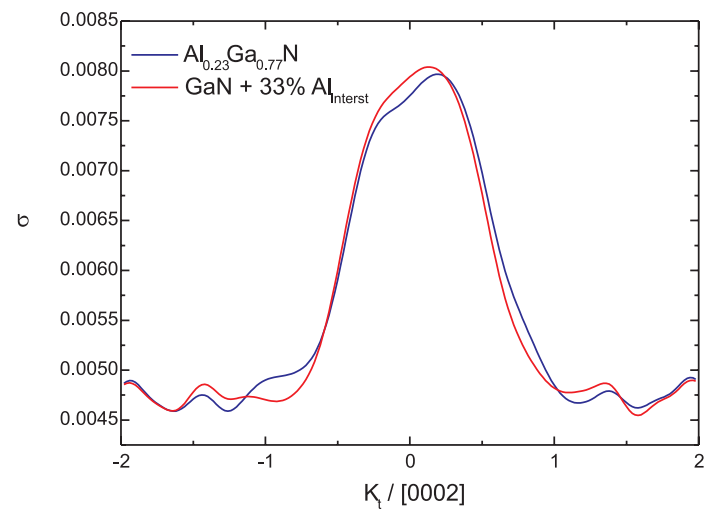

Interstitielles Al-Signal

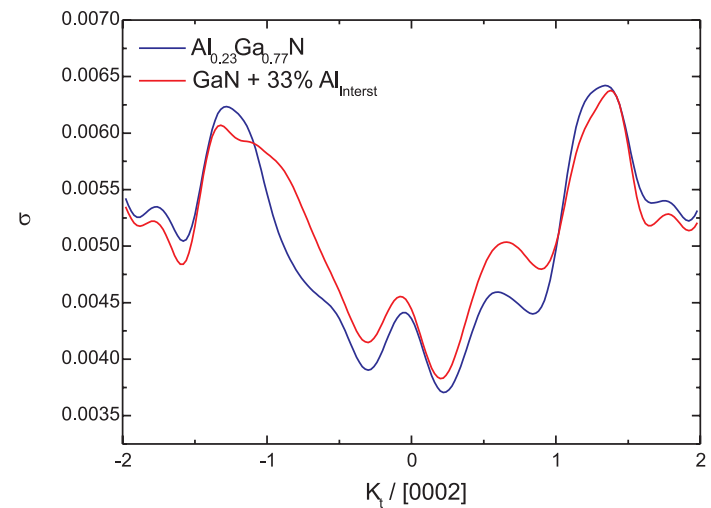

Abbildung 7.7: Vergleich zwischen den simulierten Ionisierungsstreuquerschnitte $\sigma$ für $\mathrm{Al}_{0.23} \mathrm{Ga}_{0.77} \mathrm{~N}$ und

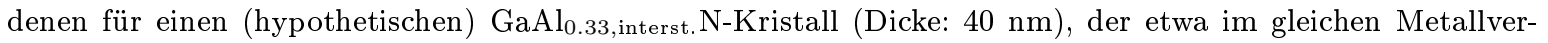
hältnis die Al-Atome unter Verletzung der Stöchiometrie interstitiell eingebaut hat. Die größten Abweichungen liegen im Bereich von 10\%, das gemessene Al-Signal hat einen relativen Fehler von etwa 20\%. Die gezeigten Simulationen berücksichtigen nicht die Strahlkonvergenz.

Gitterplatzbesetzung für das $\mathrm{Al}$ ergab, dass sich $99.9 \pm 8.9 \%$ des $\mathrm{Al}$ auf dem substitutionellen Ga-Platz befinden. Diese Bestimmung ergab einen $\chi^{2}$-Wert von 10.05 bei 10 Freiheitsgraden. Während die Besetzung das erwartete Ergebnis lieferte, lässt der hohe $\chi^{2}$-Wert vermuten, dass die Korrektur der GaN bzw. AlGaN-Anteile im beleuchteten Bereich nicht ausgereicht hat, um diesen systematischen Messfehler zu korrigieren. Bei den späteren Mn-Bestimmungen ergeben sich zwei- bis dreimal kleinere $\chi^{2}$-Werte.

Insgesamt entsprechen die experimentellen Ergebnisse den theoretischen Überlegungen, so dass die vorgestellte Methode zuverlässig zur Gitterplatzbestimmung von Mn in GaN:Mn genutzt werden kann. Dabei enthielt diese Messung noch weitere Fehlerquellen, die bei der Messung im GaN:Mn entfallen.

\subsection{Gitterplatz des $\mathrm{Mn}$ in GaN}

Um zu untersuchen, zu welchem Anteil die Mn-Atome im Kationen-Untergitter des Wurtzitkristalls eingebaut werden, wurden ALCHEMI-Untersuchungen an Probe J0141 vorgenommen. Wie in Abschnitt 6.2 beschrieben, liegt das Mn in dieser Probe gelöst vor.

In der Probe kommt es zu einem säulenartigen Wachstum des GaN (s. Abb. 7.9). Jede dieser Säulen weist dabei eine leicht andere Orientierung auf. Da das Elektronenchanneling sehr sensitiv auf Orientierungsunterschiede ist, wurde bei den Untersuchungen jeweils eine einzige Säule beleuchtet und darauf geachtet, dass im beleuchteten Bereich keine Korngrenzen oder Fadenversetzungen lagen. Hier bietet die Ortsaufösung der ALCHEMI-Technik einen großen Vorteil gegenüber Methoden wie RBS oder EXAFS, deren Signale aus großen Probenbereichen kommen.

Die mittlere Mn-Konzentration dieser Proben betrug etwa 1.5 at\%, allerdings schwankt die Mn-Konzentration im Bereich unter 5 at\% mit dem Ort innerhalb der Säulen. Der beleuchtete 
(a) Ga-Signal

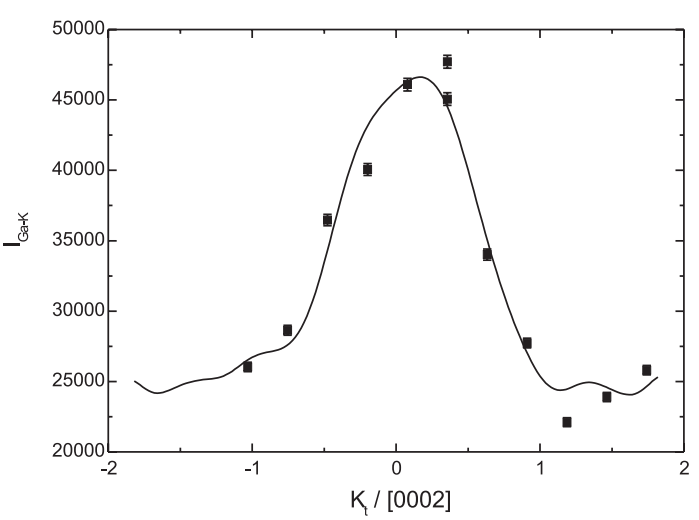

(c) $\mathrm{Al} / \mathrm{Ga}-$ Verhältnis

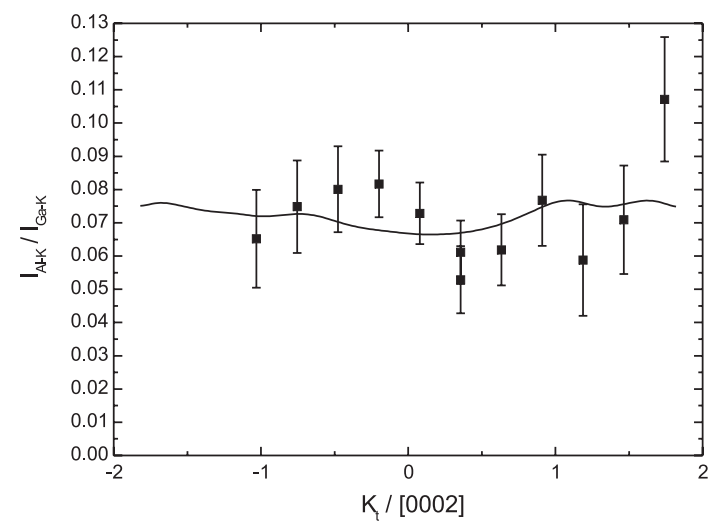

(b) Al-Signal

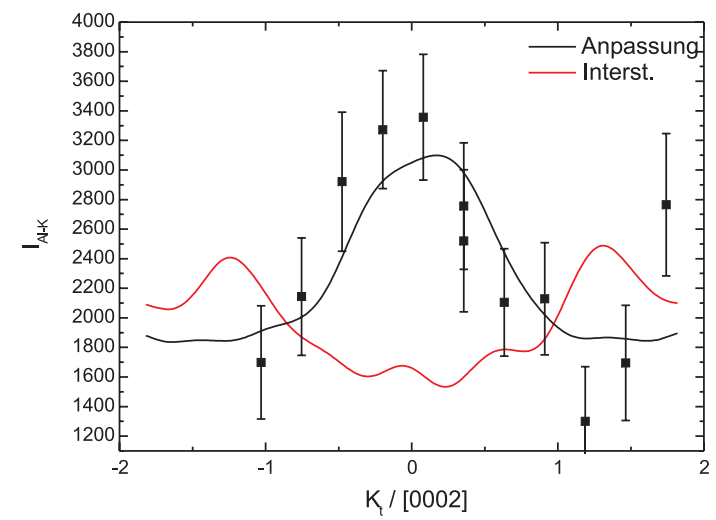

Abbildung 7.8: (a) Gemessenes (Punkte) sowie simuliertes Ga-Profil (Kurve). Die Anpassung ergab eine Probendicke von $41 \mathrm{~nm}$. (b) Gemessenes (Punkte) sowie simuliertes Al-Profil (Kurven). Die Anpassung (schwarze Kurve) ergab, dass $99.9 \pm 8.5 \%$ des Al substitutionell gelöst sind. Zusätzlich ist das simulierte Profil für den rein interstitiellen Einbau (rote Kurve) gezeigt. (c) Gemessenes (Punkte) und angepasstes simuliertes

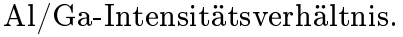


Bereich für die Messung eines Röntgenprofils ist in Abb. 7.9 markiert.

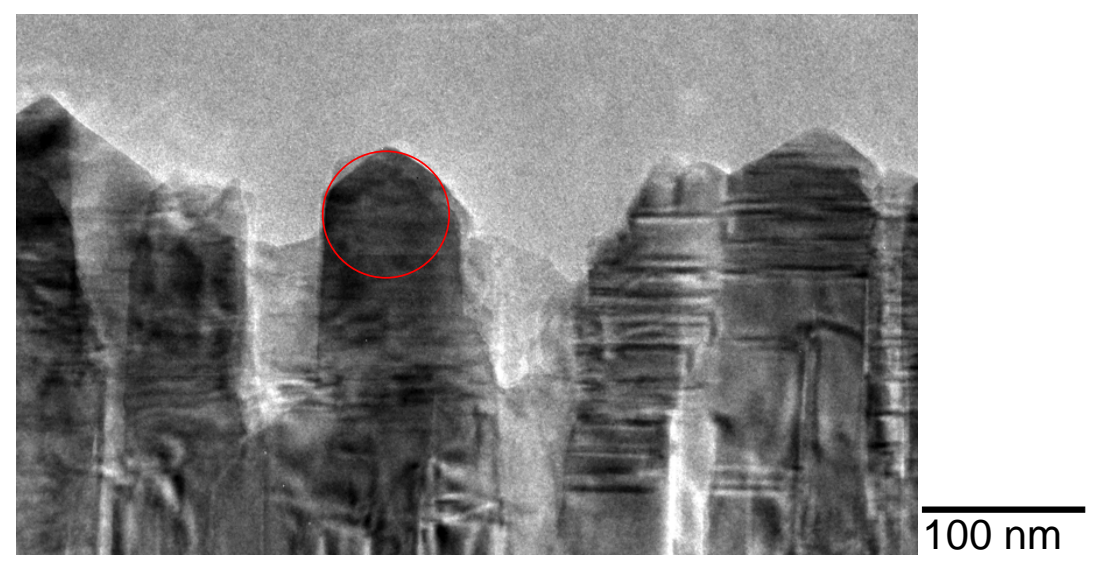

Abbildung 7.9: Übersicht des beleuchteten Bereichs für die ALCHEMI-Untersuchung (Messung B).

Es wurden an zwei unterschiedlichen Orten der Probe ALCHEMI-Messungen an einer systematischen Reihe von (0002)-Reflexen durchgeführt, die erhaltenen Röntgenprofile und ihre Anpassung sind in Abb. 7.10 gezeigt. Die Ergebnisse der Anpassungen sind in der folgenden Tabelle zusammengefasst. Die Messungen zeigen, dass das Mn vorwiegend substitutionell gelöst ist.

\begin{tabular}{ccccc} 
Messung & Dicke & Anteil subst. Mn & $\chi^{2}$ & Freiheitsgrade \\
\hline A & $80 \mathrm{~nm}$ & $95.5 \pm 6.8$ at\% & 5.39 & 13 \\
B & $48 \mathrm{~nm}$ & $95.9 \pm 6.3$ at\% & 3.77 & 13
\end{tabular}

Eine weitere Messung wurde mit einer systematischen Reihe von (11̄̄0)-Reflexen durchgeführt. Die untersuchten interstitiellen Plätze (Tetraeder- und Oktaeder-Lücke) sowie die Plätze des Kationen-Untergitters sind in dieser Reihe nicht mittels ALCHEMI-Experimenten zu unterscheiden. Die Röntgenprofile dieser drei Plätze sind identisch. Daher wird hier ausschließlich das substitutionelle Profil durch Variation der Proportionalitätskonstante an das Intensitätsverhältnis angepasst. Diese Messung soll sicherstellen, dass keine anderen Gitterplätze als die berücksichtigten für die Mn-Atome in Frage kommen. Das Ergebnis ist gemeinsam mit der Anpassung in Abb. 7.11 gezeigt. Die Anpassung ergab einen $\chi^{2}$-Wert von 2.86 (bei 13 Freiheitsgraden), was als hinreichende Bestätigung der Annahme, nur diese drei Gitterplätze zu berücksichtigen, zu werten ist. Die Abweichungen der simulierten von den gemessenen Profilen in Abb. 7.11a und 7.11b sind wahrscheinlich darauf zurückzuführen, dass bei der Messung nicht nur Reflexe der systematische Reihe, sondern auch die Reflexe in benachbarten Reihen leicht angeregt wurden. Die leichten Variationen in dem simulierten Mn/Ga-Verhältnis rühren von Delokalisierungseffekten her.

Das Ergebnis, dass das Mn vorwiegend substitutionell in GaN eingebaut wird, steht im Einklang zu Messungen anderer Gruppen, die den Gitterplatz des eingebauten Mn beobachtet haben. Messungen, die mittels EXAFS durchgeführt wurden, zeigen eine große Übereinstimmung zwischen den gemessenen Signalen und den für den substitutionellen Einbau erwarteten 
(a) Ga-Signal Messung A

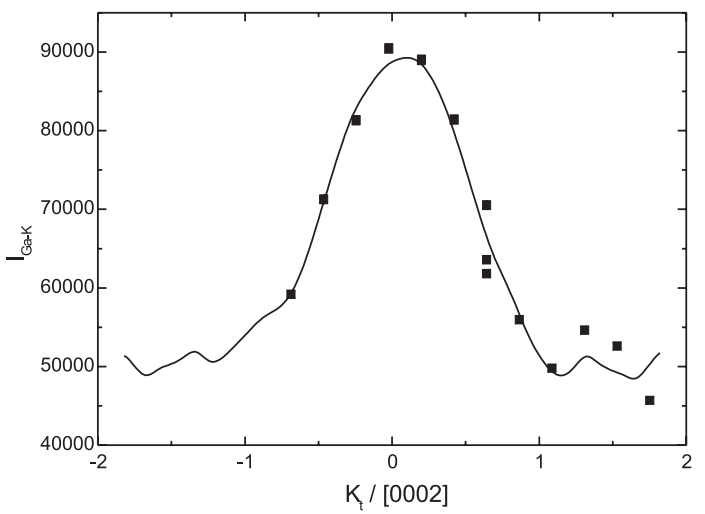

(b) Mn-Signal Messung A

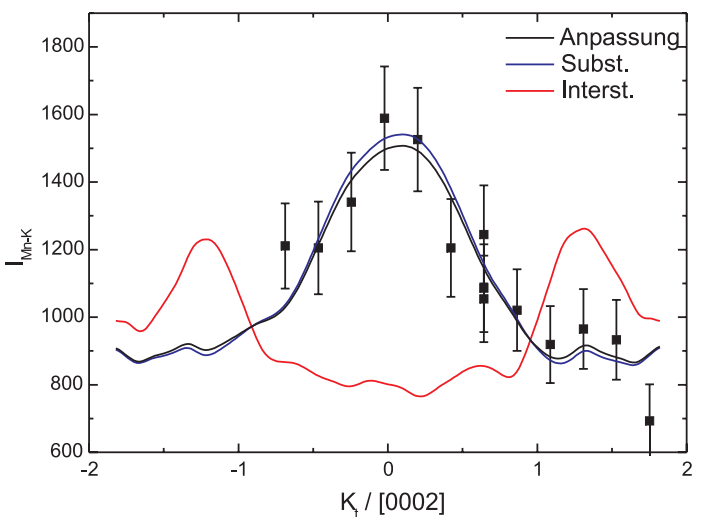

(c) Mn/Ga-Verhältnis Messung A

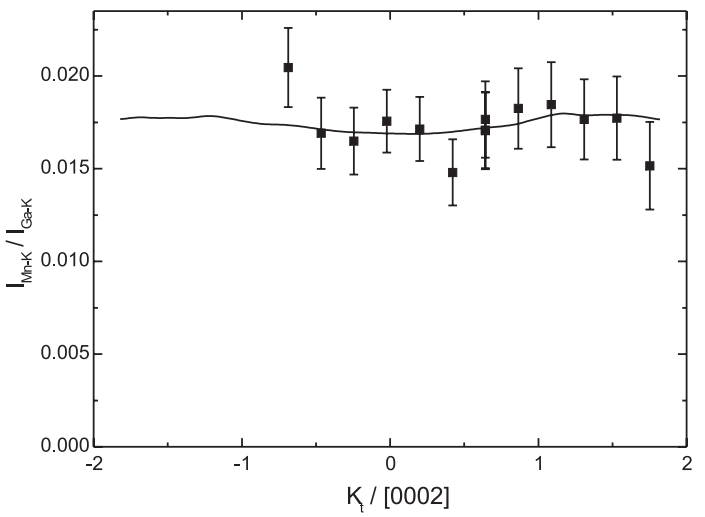

Ga-Signal Messung B

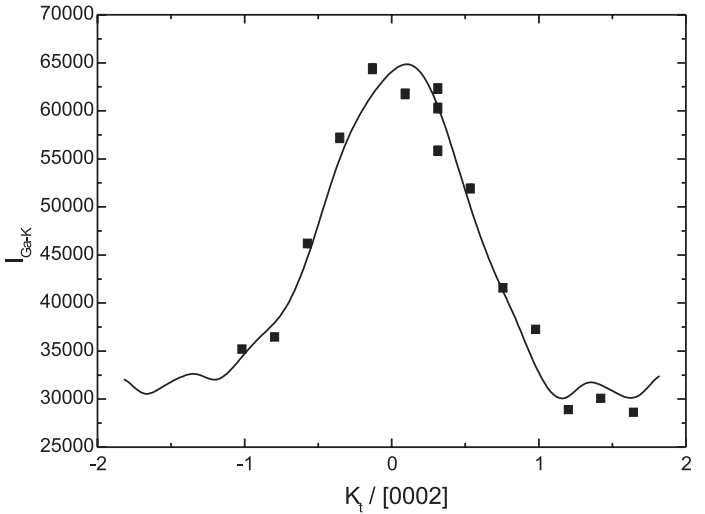

Mn-Signal Messung B

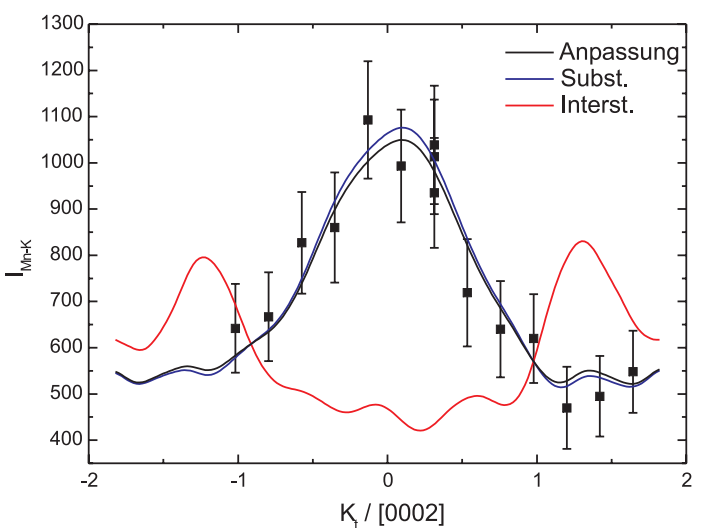

Mn/Ga-Verhältnis Messung B

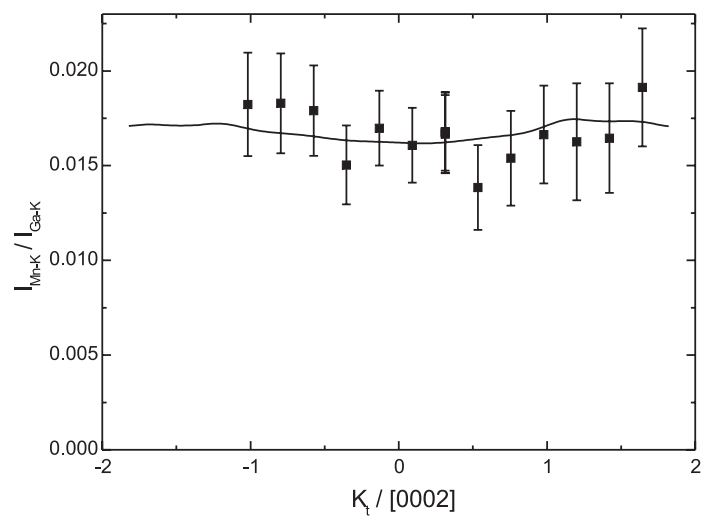

Abbildung 7.10: ALCHEMI-Messung an einer systematischen Reihe von (0002) Reflexen, s. Text für die zugehörigen Parameter: (a) Gemessene Ga-Profile und ihre Anpassung. (b) Mn Profil, die Anpassung sowie die simulierten Profile für substitutionelles und interstitielles Mn. (c) Mn/Ga Verhältnisse der Röntgenintensitäten und Anpassung. 
(a) Ga-Signal

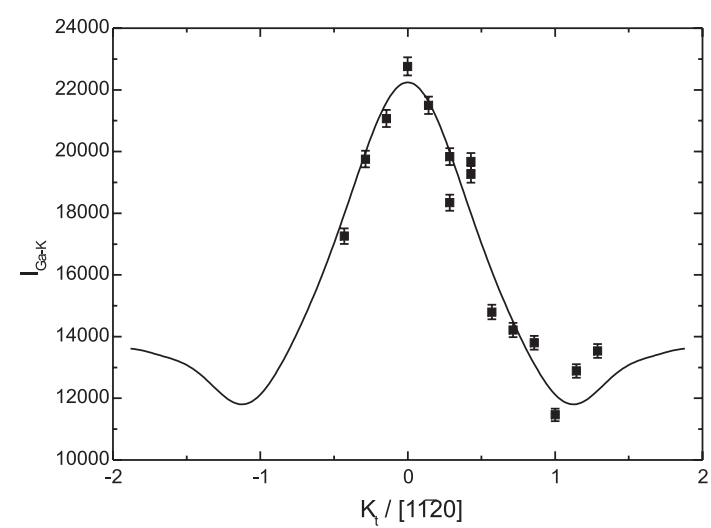

(c) $\mathrm{Mn} / \mathrm{Ga}-$ Verhältnis

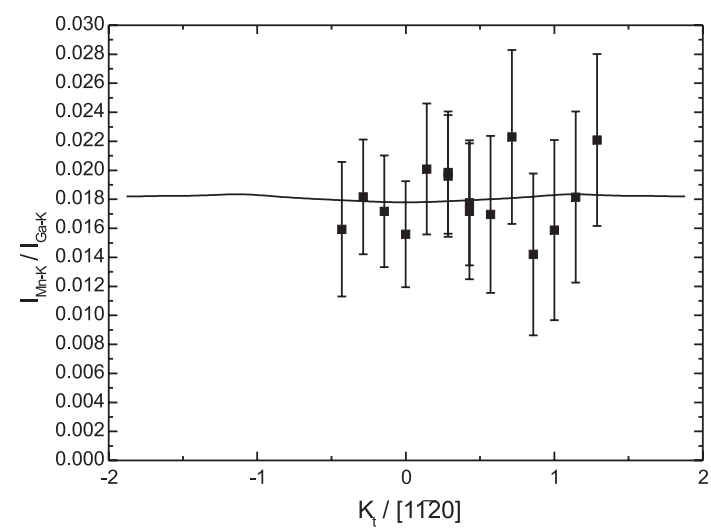

(b) Mn-Signal

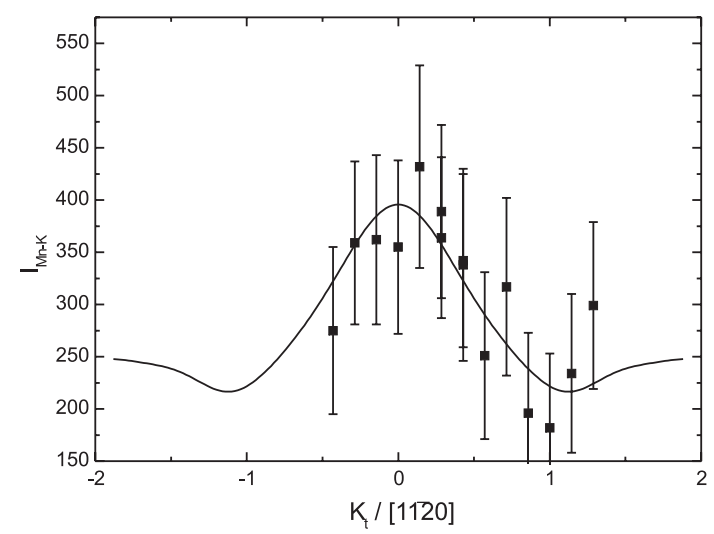

Abbildung 7.11: ALCHEMI-Messung an einer systematischen Reihe von (1120)-Reflexen. (a) Gemessenenes und angepasstes simuliertes Ga-Profil für eine Dicke von $9 \mathrm{~nm}$. (b) Gemessenes und angepasstes Mn-Profil. (c) $\mathrm{Mn} / \mathrm{Ga}$ Verhältnis der Röntgensignale und Anpassung. 
Signale [53, 54, 55]. Allerdings werden diese Messungen von keinem der Autoren auf die Größe des substitutionell eingebauten Anteils untersucht und auch nicht mit dem zu erwartenen Signal für interstitiell eingebautes Mn verglichen. Einzig Soo et al. tragen diesem Umstand Rechung und interpretieren ihre Auswertung so, dass das Mn ,größtenteils“ substitutionell eingebaut wird. Die einzigen quantitativen Messungen haben Kuroda et al. [56] mittels winkelaufgelöstem RBS durchgeführt. Sie kamen zu dem Ergebnis, dass das Mn zu 87\% - 94\% auf dem Ga-Platz zu finden ist, was im Bereich des Fehlers der hier gezeigten Messungen liegt. Einen Fehler für den von ihnen gefundenen Anteil geben Kuroda et al. nicht an.

Der hier beobachtete substitutionelle Mn-Einbau ist konsistent mit optischen Messungen an der untersuchten Probe. Es wurde eine Photoluminszenz-Linie bei $1.4 \mathrm{eV}$ gefunden, die einem intra-atomaren Übergang zwischen den kristallfeldaufgespaltenen $3 d$-Niveaus der Mn-Atome zugeordnet werden konnte [141]. Dieser Übergang ist nur bei substitionell eingebautem Mn zu finden, da er für interstitielle Mn-Atome dipol-verboten ist [50]. 


\section{Zusammenfassende Diskussion und Ausblick}

In dieser Arbeit wurden verschiedene Methoden der Transmissionselektronenmikroskopie vorgestellt und entwickelt, um dünne Schichten, wie hier GaN:Mn, in Hinblick auf auftretende ausgedehnte Defekte, ihre Mikrostruktur sowie die Verteilung und Gitterplatzbesetzung von Dotieratomen zu untersuchen.

Für die Untersuchung ausgedehnter Defekte ist eine Auflösung im atomaren Bereich nötig. Konventionelle HRTEM-Mikroskope sind durch die Linsenfehler in ihrer Punktauflösung beschränkt, obwohl diese Mikroskope auch Bildinformationen unterhalb dieser Auflösung abbilden können. Diese Bildinformationen sind allerdings durch die optische Delokalisierung, die aufgrund von Linsenfehlern und der Betragsbildung im Abbildungsprozess auftreten, nicht direkt interpretierbar. Um diese Informationen zu nutzen, müssen die Aufnahmen mit Bildsimulationen, die auf der Basis von Strukturmodellen angefertigt werden können, verglichen werden.

Mit der Objektwellenrekonstruktion, wie sie in Kapitel 4 vorgestellt wurde, wird es möglich, die den HRTEM-Abbildungen zugrundeliegende Objektwelle zu erlangen. Diese Objektwelle ist frei von optischer Delokalisierung und im Gegensatz zur reellen Abbildung eine komplexe Größe, die sowohl in der Amplitude als auch in der Phase Informationen über das Objekt enthält. In dieser Arbeit konnten so mit einem Mikroskop, das eine Punktauflösung von $1.9 \AA$ besitzt, Objektwellen rekonstruiert werden, in denen z.B. Si-Dumbbells (Abstand $1.36 \AA$; Abb. 4.9) oder sogar die Polarität von GaN-Dumbbells (Abstand der Atome 1.1 $\AA$; Abb. 5.17) erkennbar waren. Die rekonstruierten Objektwellen sind nur noch durch die Stabilität des verwendeten Mikroskops in der Aufösung begrenzt. Auch wurde es möglich, leichte Atome wie $\mathrm{N}$ trotz der Nähe von stärker streuenden Atomen wie Ga abzubilden.

Um eine Objektwellenrekonstruktion durchzuführen, sind verschiedene Verfahren nötig, die im Laufe dieser Arbeit entwickelt wurden. So mussten zunächst experimentelle Bedingungen gefunden werden, die es ermöglichen, eine Defokusserie aufzunehmen, aus der mittels Defokusserienrekonstruktion die Bildwelle rekonstruiert werden kann. Die Anwendung des Rekonstruktionsverfahrens auf Materialien wie GaN, deren Gitter keine Inversionszentren besitzen, benötigte eine besondere Methode zur Ausrichtung einzelner Abbildungen einer Serie, wie sie ebenfalls in dieser Arbeit entwickelt wurde.

Um die Linsenfehler, wie sie noch in der Bildwelle vorhanden sind, numerisch zu korrigieren, müssen die Größen dieser Linsenfehler mit einer hinreichend hohen Genauigkeit gemessen werden. Dabei sind Aberrationen wie der zweizählige Astigmatismus oder die axiale Koma, sehr sensitiv auf die konkreten Bedingungen der Abbildungen und ändern sich fortwährend, so dass sie für jede Defokusserie einzeln bestimmt werden müssen. Im Rahmen dieser Arbeit wurde ein Verfahren entwickelt, mit dem es möglich ist, diese Aberrationen mit der nötigen Genauigkeit zu ermitteln. Dies beseitigt eine mögliche Fehlerquelle in der Objektwellenrekonstruktion, 
denn es ist ansonsten üblich, die Linsenfehler nur grob zu messen, und die Feinkorrektur durch Vergleich der Objektwellen mit Simulationen durchzuführen [60, 142]. Dabei ist allerdings noch nicht untersucht, inwieweit dieser Vergleich überhaupt gültig ist. So könnte beispielsweise eine Probenverkippung einen ähnlichen Einfluss wie die axiale Koma auf die Objektwelle haben, wie es z.B. von HRTEM-Abbildungen bekannt ist [104].

Die Korrektur der optischen Delokalisierung erlaubt auch, die Geometrische Phasen-Analyse (GPA) ohne Rücksicht auf die Abbildungsbedingungen einzusetzen. In dieser Arbeit wurde erstmals die GPA auf die komplexe Objektwelle angewendet. So konnten, wie in Kapitel 5 gezeigt wird, die Verschiebungs- und Verzerrungsfelder von verschiedenen ausgedehnten Defekten gemessen werden. In konventionellen Abbildungen ist die Messung dieser Felder ein höchst anspruchvolles Problem [12], während diese Felder durch die Anwendung der GPA auf Objektwellen in einer einfachen Weise ermittelt werden können.

Die direkte Interpretierbarkeit der Objektwellen ermöglicht es, ausgedehnte Defekte auf eine neue Art und Weise zu untersuchen. So wurde in dieser Arbeit gezeigt, dass es direkt möglich ist, die Positionen von Partialversetzungen anzugeben, was beispielsweise ermöglicht, Stapelfehlerenergien zu messen. Auch können verschiedene Typen von basalen Stapelfehlern direkt voneinander unterschieden werden, wie in Abschnitt 5.3 gezeigt wurde. Weiterhin konnten die Reaktionen von Partialversetzungen mit Stapelfehlern, sei es die Aufspaltung von perfekten Versetzungen (Abschnitt 5.4 und 5.7) oder die Partialversetzungen zwischen verschiedenen Stapelfehlern (Abschnitt 5.8) direkt beobachtet werden. Auch konnten in dieser Arbeit zum ersten Mal die Kernstrukturen dieser Partialversetzungen abgebildet werden. Die direkte Interpretierbarkeit der Objektwelle ermöglicht es sogar, die Unterschiede von verschiedenen Atompositionen im Kern gegenüber den von theoretischer Seite vorgeschlagenen Strukturen aufzuzeigen.

Ferner ergeben sich durch die Kombination von GPA mit der Objektwellenrekonstruktion auch neue Methoden, selbst schräg in der Probe liegende Defekte zu untersuchen, was erstmals in dieser Arbeit demonstriert wurde. So kann (s. Abschnitt 5.6) das Verschiebungsprofil der GPA genutzt werden, um auch die Typen von prismatischen Stapelfehlern in GaN zu unterscheiden.

Durch die Analyse der Objektwellen anstatt der Intensitätsabbildungen ergeben sich eine Vielzahl weiterer Möglichkeiten der quantitativen Elektronenmikroskopie: Die Phase einer einzelnen projizierten Atomsäule in Gitterabbildungen ist sensitiv sowohl auf die Zusammensetzung als auch auf die Probendicke am Ort dieser Säule [143]. Dies könnte genutzt werden, um mit den Methoden der diskreten Tomographie dreidimensional atomare Strukturen zu analysisieren. Andere Möglichkeiten sind beispielsweise eine numerische Nanobereichsbeugung, bei der die Fouriertransformation eines Ausschnittes der Abbildung analysiert wird [60]. Diese Technik konnte bereits genutzt werden, um die radiale Paar-Verteilungsfunktion einer amorphen Korngrenze zu analysieren [144].

Um das Wachstum Mn-dotierter GaN-Schichten zu optimieren, ist neben der Analyse der Kristalldefekte auch das Einbauverhalten des Mn zu analysieren. So wurden in Abschnitt 6.1 die Methoden der abbildenden und analytischen Elektronenmikroskopie genutzt, um die Phase der auftretenden Ausscheidungen in Mn dotiertem $\mathrm{GaN}$ als $\mathrm{GaMn}_{3} \mathrm{~N}$ zu identifiziert. Zur Identifizierung dieser Ausscheidung reichen strukturelle Untersuchungen alleine nicht aus, daher müssen weitere Methoden zur Identifizierung eingesetzt werden. In der Literatur wurde diese Phase bisher nur indirekt, durch Korrelation der Ergebnisse von Röntgendiffraktometrie mit magnetischen [35] oder EXAFS-Messungen [47] bestimmt. In dieser Arbeit konnte erstmals 
durch die hohe Ortsauflösung, mit der chemische Analysen im TEM möglich sind, direkt die Phase aus der Struktur und der Zusammensetzung von ein- und derselben Ausscheidung bestimmt werden. Auch wurde festgestellt, dass bei Auscheidungsbildung der Mn-Gehalt in der GaN-Matrix unter Wachstumsbedingungen nahe am Stöchiometrischen Punkt unterhalb der EDX-Nachweisgrenze $(\approx 1$ at $\%)$ lag.

Für den Fall, das das Mn gelöst in den Proben vorliegt, wurde in dieser Arbeit erstmals die Homogenität der Mn-Verteilung untersucht. Dabei wurde eine inhomogene Verteilung des Mn beobachtet. Das Mn sammelte sich bevorzugt in der Mitte der inselartigen Strukturen in der Probe, die bei einem Mn-Angebot von 15 at\% gewachsen wurden (s. Abb. 6.18). Noch deutlicher wurde dies in einer Probe, bei der nur 4 at\% Mn angeboten wurden und die GaN-Schicht säulenartig wuchs. Hier fand sich das Mn in Bereichen, die am weitesten von den Oberflächen der Säulen entfernt waren (s. Abb. 6.13). Dabei konnten in den Mn-reichen Bereichen Konzentrationen von bis zu über 7 at\% nachgewiesen werden, während die Konzentrationen der Mn-armen Bereiche teilweise unterhalb der Nachweisgrenze lagen. Durch die Möglichkeit mittels der „Focused Ion Beam“-Technik auch Proben in schwierigen Geometrien, wie hier eine Aufsichtprobe von einer $400 \mathrm{~nm}$ dünnen Schicht, herzustellen, konnte diese Mn-Verteilung innerhalb der Säulen noch deutlicher beobachtet werden.

Um zu untersuchen, zu welchen Anteilen das gelöste Mn auf substitutionellen Gitterplätzen eingebaut wird, wurde eine erweiterte ALCHEMI-Technik entwickelt (Kapitel 7). Diese Anteile werden durch den Vergleich von gemessenen winkelaufgelösten EDX-Profilen mit angepassten Simulationen bestimmen. Dabei wurde gefunden, dass sich das Mn zu $96 \pm 6 \%$ auf dem Kationen-Gitterplatz befindet. Im Gegensatz zu anderen Untersuchungen des MnGitterplatzes, wie sie in der Literatur zu finden sind, ist es mit dieser Methode möglich, die Mikrostruktur der Probe zu berücksichtigten, da diese Untersuchung mit hoher Ortsauflösung durchgeführt werden kann. Weil die ausgenutzten Channeling-Effekte sehr sensitiv auf den Einstrahlwinkel sind, wäre eine derartige winkelabhängige Messung mit anderen Methoden in den säulenartigen Proben aufgrund der leichten Verdrehung der Säulen gegeneinander unmöglich.

Die gemessene inhomogene Mn-Verteilung könnte einen Hinweis auf die Ursachen der Diskrepanz zwischen den theoretischen Modellen und experimentellen Beobachtungen bzgl. des Ferromagnetismus von GaN:Mn geben. Wie neuere theoretische Arbeiten vorschlagen, könnte sich das Mn durch eine spinodale Entmischung in bestimmten Bereichen anreichern, in denen sich dann Mn-Cluster oder Nanokristalle in Form von wurzitischen MnN bilden. Diese Cluster bzw. Kristalle könnten dann die Quellen des experimentell beobachteten Ferromagnetismus sein. Diese Entmischung könnte sowohl aufgrund von Coulumb-Kräften, wie Dietl in einem aktuellen Artikel [145] vorschlägt, oder aufgrund von Austauschwechselwirkung zwischen den Mn-Ionen auftreten [40, 146].

Die Anreicherung, wie sie hier beobachtet wird, lässt noch einen anderen Grund für eine solche Entmischung möglich erscheinen: im System GaN ist bekannt, dass Spannungsfelder den Einbau von Dotieratomen beeinflussen können [147]. Da Spannungsfelder an Oberflächen verschwinden, ist anzunehmen, dass sie am größten in Bereichen sind, die weit von der Oberfläche der Inseln bzw. Säulen entfernt sind. Gerade in diesen Bereichen wird eine Mn-Anreicherung gefunden.

Es zeigt sich, dass das System GaN:Mn sehr komplex ist, da neben den angesprochenen Verzerrungsfeldern sich auch weitere Einflüsse z.B. die Lage des Ferminiveaus [148] auf den 
Mn-Einbau auswirken. Dies eröffnet allerdings auch neue Möglichkeiten für den Optimierungsprozess. So könnte es möglich werden, mit Hilfe dieser Wechselwirkungen lokal MnAnreicherungen im Bereich des für den Ferromagnetismus nötigen Perkolationslimits zu erreichen. Es bleibt noch die Frage offen, ob das Mn sich in Clustern oder wurzitischen Nanoausscheidungen ansammelt. Methoden, wie z.B. ELNES, die sensitiv auf die chemische Umgebung der Mn-Atome sind, könnten evtl. MnN-Cluster nachweisen. Wurzitische MnN-Phasen könnten unter Umständen auch mittels Objektwellenrekonstruktion durch die Sensitivität der Phase der Objektwelle auf chemische Unterschiede direkt nachgewiesen werden.

Wie diese Arbeit zeigt, erlaubt die moderne Transmissionselektronenmikroskopie eine sehr weitreichende Charakterisierung der GaN-Schichten. So ergeben sich durch die Objektwellenrekonstruktion neue Möglichkeiten zur Analyse ausgedehnter Defekte, da z.B. die Kernstrukturen von Versetzungen direkt beobachtet werden können. Auch die Verzerrungsfelder der Defekte lassen sich auf einfache Weise messen. Mit der ALCHEMI-Technik wird es möglich, die Gitterplätze von Dotieratomen zu untersuchen. Eine inhomogene Mn-Verteilung, wie sie in dieser Arbeit beobachtet wurde, ist ein weiterer Effekt im System GaN:Mn, der bei weiteren Untersuchungen von GaN:Mn-Schichten sicherlich berücksichtigt werden muss. Eine ausführliche strukturelle Charakterisierung der Proben wie sie mit den hier vorgestellten Methoden möglich ist, ist sicherlich ein nötiger Schritt, um die Frage nach den Mechanismen des Ferromagnetismus in Mn-dotierten $\mathrm{GaN}$ zu beantworten. 


\section{A Kalibrierung der Strahlverkippung}

In dieser Arbeit wird an verschiedenen Stellen die Möglichkeit genutzt, den Elektronenstrahl des Mikroskops zu verkippen: zum einen bei der Bestimmung der Aberrationskonstanten (s. Abschnitt 4.3), zum anderen bei den ALCHEMI-Experimenten (s. Kapitel 7). In beiden Fällen ist die Kenntnis der genauen Kippwinkel wichtig. Für die Messung der Aberrationskonstanten ist es zusätzlich notwendig, diese im Koordinatensystem der CCD-Kamera anzugeben, d.h. im Koordinatensystem der benutzten HRTEM-Abbildungen (hier 560000-fache Vergrößerung).

Anhand des in Abb. A.1 skizzierten Strahlengangs wird das Vorgehen erläutert. Der Elektronenstrahl wird von den Ablenkspulen in einem Winkel $(u, v)$ zur optischen Achse abgelenkt. Am Mikroskop selbst werden diese Winkel auf einer Skala angegeben ${ }^{1}$, die in diesem Abschnitt kalibriert werden soll. Der Elektronenstrahl trifft auf das Objekt, von dem neben dem unabgelenkten Strahl auch um den Bragg-Winkel $\lambda \boldsymbol{g}$ abgelenkte Strahlen ausgehen.

Im Beugungsbild entsprechen diese Winkel $(u, v)$ und $\lambda \boldsymbol{g}$ den Strecken $\boldsymbol{a}$ bzw. $\Delta \boldsymbol{a}$. Da die Abbildung selbst (s. Abschnitt 3.1.2.1) ein Interferenzmuster zwischen verschiedenen Strahlen ist, lassen sich nur Winkel zwischen Strahlen messen. Diese Winkel $\Delta t$ entsprechen dabei Strecken im Diffraktogramm. Durch die Funktionsweise von Elektronenlinsen kommt es zusätzlich zu einer Drehung um die optische Achse zwischen den drei benutzten Koordinatensystemen, in der Abbildung, im Beugungsbild und in der Ebene der Ablenkspulen.

Bei kristallinen Objekten sind die Winkel $\lambda \boldsymbol{g}$ der Reflexe bekannt, so dass sie benutzt werden können um $\Delta \boldsymbol{a}$ und $\Delta t$ zu kalibrieren. Aus ihnen lässt sich dann eine Umrechnung von der Skala $(u, v)$ ins Koordinatensystem der Abbildung $t$ angeben.

\section{A.1 Strahlverkippung im Beugungsbild}

Zur Kalibrierung im Beugungsbild wurde eine Probe verwendet, bei der sich viele kleine Goldkristalle auf einem Kohlenstofffilm befinden. Die Beugungsbilder wurden von einem großen Probenbereich unter gleichbleibenden Bedingungen aufgenommen. Durch die vielen Kristalle besteht das Beugungsbild aus einem konzentrischen Ringmuster. Aus dem Durchmesser der Ringe, die den Braggreflexen der Gold- $f c c$ Struktur zugeordnet werden können, wurde zunächst das Beugungsbild kalibriert.

Das Zentrum der Ringe entspricht dem unabgelenkten Strahl, es kann dadurch bestimmt werden, dass die Gesamtvarianz der azimuthalen Mittelung minimiert wird: Für die azimuthale Mittelung muss ein Mittelpunkt angegeben werden bzgl. dessen die Mittelung vorgenommen

\footnotetext{
${ }^{1}$ Am Mikroskop selbst werden die Winkel auf dieser Skala in Grad angegeben. Da Um keine Verwechslungsgefahr zwischen diesen nominellen Winkeln und den wahren Winkeln aufkommen zu lassen, wird im Folgenden von Skalenteilen gesprochen. Am Mikroskop werden $u$ als Verkippung in X-Richtung und $v$ als Verkippung in Y-Richtung bezeichnet.
} 


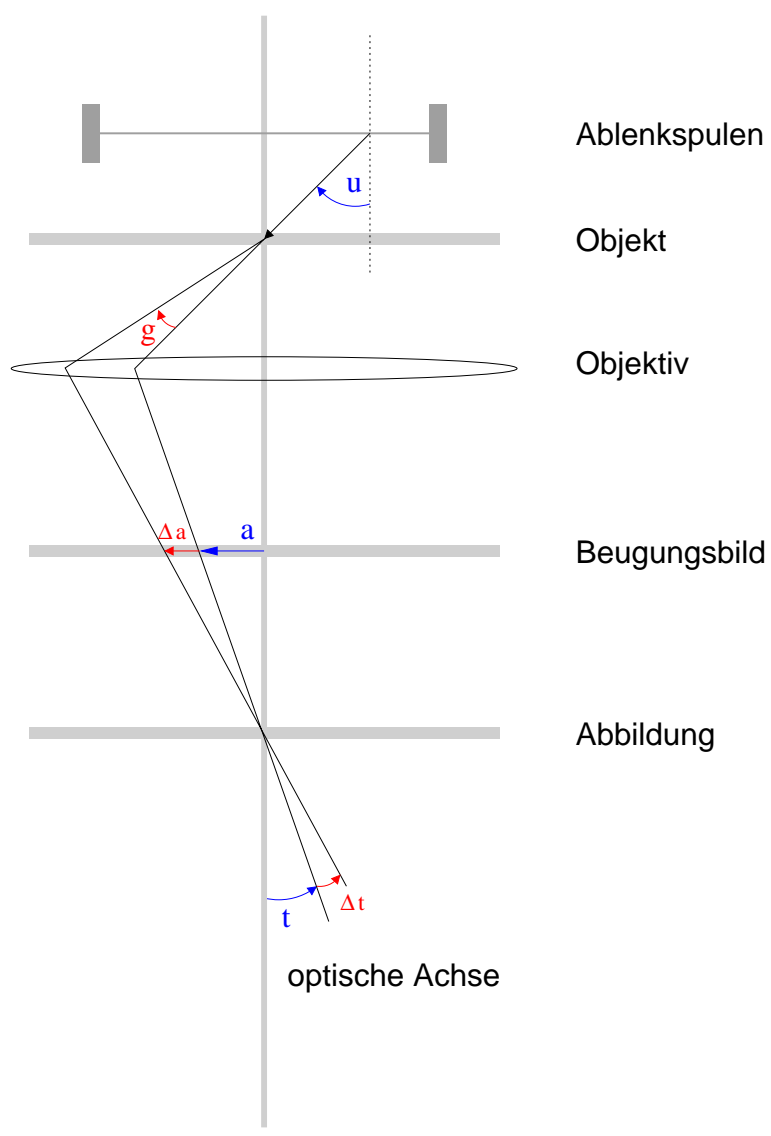

Abbildung A.1: Strahlengang für die Kalibrierung der Strahlverkippung. Die Verkippung $u$ bzw. die Braggwinkel $g$ entsprechen Strecken $a$ bzw $\Delta a$ im Beugungsbild. In der Abbildung können nur Winkel $\Delta t$ (d.h. Strecken im Diffraktogramm) zwischen den Strahlen gemessen werden. 
wird. Entspricht dieser Mittelpunkt dem Zentrum eines rotationssymmetrischen Musters, wird die Gesamtvarianz der Mittelung minimal.

Für verschiedene Kippwinkel gegen die optische Achse (0.5 Skt, 1.0 Skt und 1.5 Skt mit je $45^{\circ}$ azimuthaler Schrittweite) wurden Beugungsbilder aufgenommen. Außerdem während der Messung mehrere Aufnahmen ohne Strahlverkippung gemacht. Eine Strahlverkippung entspricht einer Verschiebung des Beugungsbildes. Für alle Beugungsbilder wurde daher das Zentrum $\left(p_{x}, p_{y}\right)$ der Beugungskreise bestimmt.

Die Position des Zentrums in sämtlichen unverkippten Beugungsbildern sollte im Prinzip gleich sein. Die Schwankung $\sigma_{\text {axial }}$ dieser Position soll daher als Abschätzung für den Fehler dienen, der durch Ungenauigkeiten bei der Bestimmung des Zentrums, Hysteresen in den Ablenkspulen und Drift der Strahlrichtung im restlichen Beleuchtungssystem während der Messung entsteht.

Zur Kalibrierung der Strahlverkippung wurde anschließend an die Koordinaten der Zentren $\left(p_{x}, p_{y}\right)$ der Beugungsbilder mit einer $\chi^{2}$-Minimierung die quadratische Form

$$
p_{x}=a_{0 ; x}+a_{u ; x} u+a_{v ; x} v+a_{u u ; x} u^{2}+a_{v v ; x} v^{2}+a_{u v ; x} u v
$$

bzw. für $p_{y}$ die analoge Form angepasst, wobei $(u, v)$ die nominelle Strahlverkippung ist. Eine Abweichung vom linearen Zusammenhang zwischen nomineller und wahrer Verkippung würde zu nichtverschwindenen quadratischen Termen führen. Die Terme $\left(a_{0 ; x}, a_{0 ; y}\right)$ geben den Durchstoßpunkt der optischen Achse $((u, v)=0)$ in der Beugungsebene an. Die Fehler der Koeffizienten wurden durch eine Monte-Carlo-Simulation mit 2000 Punkten bestimmt, in der jede Zentrumskoordinaten innerhalb einer Gaußverteilung mit $\sigma_{\text {axial }}$ variiert wurde.

Die Anpassung ergab, das die Größe der quadratischen Terme weniger als 1\% der linearen Terme beträgt, so dass die Nichtlinearitäten der Verkippung zu vernachlässigen sind. Der Winkel zwischen den beiden Strahlablenkungsrichtungen $u$ und $v$ beträgt $90.22^{\circ}$. Insgesamt lässt sich folgende Kalibrierung der Kippwinkel feststellen:

$$
1 \mathrm{Skt} \equiv 4.275 \mathrm{~nm}^{-1} \equiv 0.614^{\circ} .
$$

Die genaue Umrechnung der Strahlverkippungen $(u, v)$ in Skalenteilen in das Koordinatensystem der CCD-Abbildung $(x, y)$ in Pixeln lautet bei einer nominellen Kameralänge des Mikroskops von $190 \mathrm{~mm}$ :

$$
\left[\begin{array}{l}
x \\
y
\end{array}\right]=\left[\begin{array}{cc}
-7.77(49) & 112.34(49) \\
111.76(49) & 7.29(49)
\end{array}\right] \cdot\left[\begin{array}{l}
u \\
v
\end{array}\right]
$$

\section{A.2 Umrechnung Beugungsbild-Diffraktogramm}

Um die Umrechnung der Strahlverkippung $(u, v)$ in das Koordinatensystem der Abbildung zu bestimmen, wurden ein Beugungsbild sowie ein Diffraktogramm eines Si-Kristalls in [110]Projektion aufgenommen. In diesen Abbildungen wurden die Reflexe indiziert und ihre Koordinaten mit einem Schwerpunktverfahren [113] bestimmt. Die Richtungen und Längen zweier Basisvektoren des Beugungsmusters (hier [001] und [11̄0]) wurden in jeder Abbildung mittels einer $\chi^{2}$-Anpassung aus der Lage der Reflexe bestimmt. Aus den Basisvektoren beider Abbildungen ergibt sich die Umrechnung. 
Die Umrechnung der CCD-Koordinaten des Beugungsbildes $(x, y)$ (in Pixel) bei $190 \mathrm{~mm}$ Kameralänge in die CCD-Koordinaten eines Diffraktogramms $\left(q_{x}, q_{y}\right)$ (in Pixel) bei 560000-facher Vergrößerung und einem Ausschnitt von 1024x1024 Pixeln lautet:

$$
\left[\begin{array}{l}
q_{x} \\
q_{y}
\end{array}\right]=\left[\begin{array}{cc}
1.0430 & -0.1141 \\
0.1116 & 1.0520
\end{array}\right] \cdot\left[\begin{array}{l}
x \\
y
\end{array}\right]
$$

Da Diffraktogramme als Betrag der Fouriertransformierten einer (reellen) Abbildung immer inversionssymmetrisch sind, ist diese Koordinatentransformation nicht eindeutig. Die Transformation ist nur bis auf eine $180^{\circ}$-Drehung bzw. eine Inversion festgelegt. Im nächsten Abschnitt wird beschrieben, wie diese Doppeldeutigkeit aufgehoben werden kann.

\section{A.3 Aufhebung der Doppeldeutigkeit}

In Abschnitt 3.4.2 wurde bereits darauf verwiesen, dass eine Strahlkippung zu einer Bildverschiebung führt. Dieser Effekt soll nun dafür genutzt werden, die Doppeldeutigkeit der im letzten Abschnitt bestimmten Koordinatentransformation aufzuheben.

Für ein Zemlin-Tableau (s. Abschnitt 4.1) wurden die Verschiebungen $(\Delta x, \Delta y)$ zwischen jeweils zwei aufeinanderfolgenden Abbildungen (Unterschied von $45^{\circ}$ Azimuthalwinkel) über die Kreuzkorrelationsfunktion bestimmt. Außerdem wurde für das gesamte Tableau der axiale Defokuswert bestimmt, der den wesentlichen Anteil der Verschiebungen für den benutzten Kippwinkel ausmacht. Die erwarteten Verschiebungen für beide möglichen Koordinatentransformationen, d.h. mit und ohne Inversion, wurden berechnet.

Die Verschiebungen sind in Abb. A.2 gezeigt. Während sich der Drehsinn der Verschiebungen aus dem Drehsinn bei der Aufnahme des Tableaus ergibt, beginnt die Rotation dieser Verschiebung bei beiden Möglichkeiten bei einer anderen Position. Die gemessenen und die ohne Inversion berechneten Verschiebungen beginnen auf der 10-Uhr Position, während die erwarteten Verschiebungen bei Inversion auf der 4-Uhr Position beginnen. Die Abweichungen der gemessenen Positionen von den berechneten lassen sich durch die Probendrift während der Aufnahme des Tableaus erklären. Wiederholungen dieser Auswertung mit verschiedenen Tableaus ergaben das gleiche Resultat.

Somit kann eine zusätzliche Inversion ausgeschlossen werden.

\section{A.4 Kalibrierung}

Zusammengefasst lautet die Kalibrierung der Strahlverkippung $(u, v)$ in Skalenteilen im Koordinatensystem der CCD-Kamera $\left(q_{x}, q_{y}\right)$ in $1 / \mathrm{nm}$ bei 560000 -facher Vergrößerung und einem Sampling von $0.02658 \mathrm{~nm} / \mathrm{Pixel}$ :

$$
\left[\begin{array}{l}
q_{x} \\
q_{y}
\end{array}\right]=\left[\begin{array}{cc}
-0.766 & 4.274 \\
4.288 & 0.742
\end{array}\right] \cdot\left[\begin{array}{l}
u \\
v
\end{array}\right] .
$$


$\Delta \mathrm{x} / \mathrm{nm}$

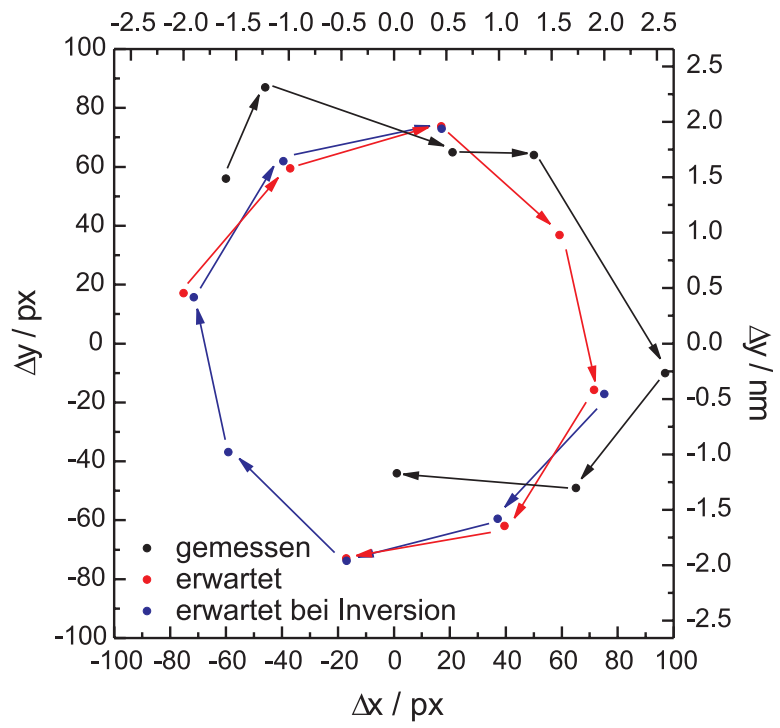

Abbildung A.2: Gemessene und berechnete Verschiebungen zwischen den Abbildungen eines Tableaus. Die gemessenen und ohne Inversion berechneten Verschiebungen beginnen ihre Rotation in einer 10-Uhr Position, mit Inversion würde die Rotation auf der 4-Uhr Position beginnen. 


\section{B Anisotrope Elastizitätstheorie von Versetzungen}

Dieser Abschnitt folgt im Wesentlichen den Ausführungen von Steeds [149] für die anisotrope Elastizitätstheorie von Versetzungen. Dabei wird auch die dortige Nomenklatur verwendet.

Die gestrichenen Tensorkomponenten und Richtungen beziehen sich auf das Standardkoordinatensystem des hexagonalen Kristalls, d.h. $x_{3}^{\prime}$ liegt parallel zur $c$-Achse und die $x_{1}^{\prime}$ und $x_{2}^{\prime}$-Richtungen in der Basalebene. Da die Basalebene elastisch isotrop ist, ist die genaue Lage der beiden Achsen beliebig. Ungestrichene Größen beziehen sich auf das Koordinatensystem der Versetzung.

Der Elastizitätstensor für Wurtzit lautet

$$
c_{i j}^{\prime}=\left[\begin{array}{cccccc}
c_{11}^{\prime} & c_{12}^{\prime} & c_{13}^{\prime} & 0 & 0 & 0 \\
c_{12}^{\prime} & c_{11}^{\prime} & c_{13}^{\prime} & 0 & 0 & 0 \\
c_{13}^{\prime} & c_{13}^{\prime} & c_{33}^{\prime} & 0 & 0 & 0 \\
0 & 0 & 0 & c_{44}^{\prime} & 0 & 0 \\
0 & 0 & 0 & 0 & c_{44}^{\prime} & 0 \\
0 & 0 & 0 & 0 & 0 & c_{66}^{\prime}
\end{array}\right]
$$

Aus der Isotropie der Basalebene folgt $c_{66}^{\prime}=\frac{1}{2}\left(c_{11}^{\prime}-c_{12}^{\prime}\right)$. Damit gibt es nur fünf unabhängige Elastizitätskonstanten.

In der Literatur sind folgende Werte für diese Konstanten zu finden:

\begin{tabular}{lccccc} 
Autor & $c_{11}^{\prime}[\mathrm{GPa}]$ & $c_{33}^{\prime}[\mathrm{GPa}]$ & $c_{44}^{\prime}[\mathrm{GPa}]$ & $c_{12}^{\prime}[\mathrm{GPa}]$ & $c_{13}^{\prime}[\mathrm{GPa}]$ \\
\hline \hline Savastenko et al.[117] & $296 \pm 18$ & $267 \pm 17$ & $24 \pm 2$ & $130 \pm 10$ & $158 \pm 5$ \\
Schwarz et al.[121] & 377 & 209 & 81.4 & 160 & 114 \\
Yamaguchi et al.[119] & $365 \pm 0.2$ & $381 \pm 0.1$ & $109 \pm 0.3$ & $135 \pm 0.4$ & $114 \pm 1.6$ \\
Takagi et al.[120] & 374 & 379 & 101 & 106 & 70 \\
Polian et al.[122] & $390 \pm 15$ & $398 \pm 20$ & $105 \pm 10$ & $145 \pm 20$ & $106 \pm 20$ \\
Wright[118] (theor.) & 367 & 405 & 95 & 135 & 103 \\
\hline
\end{tabular}

Die Werte von Savastenko et al. wurden aus der temperaturabhängigen Verbreiterung von Peaks in in Röntgenbeugungsexperimenten ${ }^{1}$, die Werte von Yamaguchi et al., Takagi et al. sowie Polian et al. aus Brillouin Streuung und die Werte von Schwarz et al. aus resonanter Überschallspektroskopie bestimmt. Die Werte von Wright hingegen sind mittels DFT/LDAMethoden berechnet worden.

\footnotetext{
${ }^{1}$ Siehe Diskussion in Abschnitt 5.4 bzw. in [118].
} 
Der zum Elastizitätstensor inverse Tensor, der Compliance-Tensor, lautet:

$$
s_{i j}^{\prime}=\left\{c_{i j}^{\prime}\right\}^{-1}=\left[\begin{array}{cccccc}
s_{11}^{\prime} & s_{12}^{\prime} & s_{13}^{\prime} & 0 & 0 & 0 \\
s_{12}^{\prime} & s_{11}^{\prime} & s_{13}^{\prime} & 0 & 0 & 0 \\
s_{13}^{\prime} & s_{13}^{\prime} & s_{33}^{\prime} & 0 & 0 & 0 \\
0 & 0 & 0 & s_{44}^{\prime} & 0 & 0 \\
0 & 0 & 0 & 0 & s_{44}^{\prime} & 0 \\
0 & 0 & 0 & 0 & 0 & s_{66}^{\prime}
\end{array}\right],
$$

wobei $s_{66}^{\prime}=2\left(s_{11}^{\prime}-s_{12}^{\prime}\right)$ ist.

Die in dieser Arbeit untersuchten Versetzungen haben ihre Versetzungslinie entlang $\langle 1 \overline{2} 10\rangle$ und $\langle 1 \overline{1} 00\rangle$-Richtungen, d.h. sie liegen beide in der Basalebene. Alle Richtungen in der Basalebene sind bzgl. ihrer elastischen Eigenschaften (allerdings nicht bzgl. ihrer atomaren Anordnung) zweizählige Symmetrieachsen, und in beiden Fällen liegt die $c$-Achse (6-fache Symmetrieachse) senkrecht zur Versetzungsrichtung. Damit lassen sich die elastischen Eigenschaften dieser Versetzungen mit den von Steeds für diesen Fall angegebenen analytischen Lösungen beschreiben.

Im Koordinatensystem der Versetzung liegt die $c$-Richtung entlang $x_{2}$. Somit entspricht die $x_{1} x_{3}$-Ebene der Basalebene des Wurtzits. Die Versetzungslinie liegt parallel zur $x_{3}$-Achse und die Versetzung befindet sich im Ursprung der $x_{1} x_{2}$-Ebene. Die Formeln sind aufgrund der elastischen Isotropie der Basalebene für alle Versetzungslinien in der Basalebene identisch. In diesem Koordinatensystem lautet der Compliance-Tensor

$$
s_{i j}=\left[\begin{array}{cccccc}
s_{11}^{\prime} & s_{13}^{\prime} & s_{12}^{\prime} & 0 & 0 & 0 \\
s_{13}^{\prime} & s_{33}^{\prime} & s_{13}^{\prime} & 0 & 0 & 0 \\
s_{12}^{\prime} & s_{13}^{\prime} & s_{11}^{\prime} & 0 & 0 & 0 \\
0 & 0 & 0 & s_{44}^{\prime} & 0 & 0 \\
0 & 0 & 0 & 0 & s_{66}^{\prime} & 0 \\
0 & 0 & 0 & 0 & 0 & s_{44}^{\prime}
\end{array}\right] .
$$

Für die Berechnung der Stufenkomponente wird der reduzierte Compliance-Tensor, der sich aus Annahme $\epsilon_{33}=0$ ergibt, benutzt:

$$
S_{i j}=s_{i j}-\frac{s_{3 i} s_{3 j}}{s_{33}} .
$$

Damit lassen sich die Hilfsgrößen

$$
\begin{aligned}
\Lambda & =-\frac{2 S_{12}+S_{66}}{2 S_{11}} \\
\lambda^{4} & =\frac{S_{22}}{S_{11}}
\end{aligned}
$$

definieren.

\section{B.1 Verschiebungsfeld}

Die Verschiebungsfelder der Stufenkomponente (Komponente des Burgersvektors in der $x_{1} x_{2}$ Ebene) und der Schraubenkomponente (Komponente des Burgersvektors entlang $x_{3}$ ) lassen 
sich unabhängig voneinander berechnen. Dabei werden die Verschiebungsfelder in Zylinderkoordinaten mit

$$
r^{2}=x_{1}^{2}+x_{2}^{2} \text { sowie } \theta=\arctan \frac{x_{2}}{x_{1}}
$$

angegeben.

Das Verschiebungsfeld der Schraubenkomponenten $b_{3}$ wird durch

$$
u_{3}=\frac{b_{3}}{2 \pi} \arctan \left[\sqrt{\frac{S_{44}}{S_{55}}} \tan \theta\right]
$$

beschrieben. Mit

$$
\begin{aligned}
A & =\arctan \frac{\sqrt{2\left(\lambda^{2}-\Lambda\right)} \tan \theta}{1-\lambda^{2} \tan ^{2} \theta} \\
B & =\frac{\left(\Lambda+S_{12} / S_{11}\right)}{\sqrt{\Lambda^{2}-\lambda^{4}}} \arctan \frac{\sqrt{-2\left(\Lambda+\lambda^{2}\right)} \tan \theta}{1+\lambda^{2} \tan ^{2} \theta} \\
C & =\frac{\left(\lambda^{2}+S_{12} / S_{11}\right)}{\sqrt{2\left(\lambda^{2}-\Lambda\right)}} \ln \left[r^{2} \sqrt{\cos ^{4} \theta+\lambda^{4} \sin ^{4} \theta-\frac{1}{2} \Lambda \sin ^{2} 2 \theta}\right] \\
D & =\frac{\left(\lambda^{2}-S_{12} / S_{11}\right)}{2 \sqrt{-2\left(\Lambda+\lambda^{2}\right)}} \ln \frac{\cos ^{2} \theta-\left(\Lambda-\sqrt{\Lambda^{2}-\lambda^{4}}\right) \sin ^{2} \theta}{\cos ^{2} \theta-\left(\Lambda+\sqrt{\Lambda^{2}-\lambda^{4}}\right) \sin ^{2} \theta}
\end{aligned}
$$

ist die Lösung für die Stufenkomponente $\left(b_{1}, b_{2}\right)$ parallel zu $x_{1}\left(b_{2}=0\right)$, d.h. der Burgersvektor liegt in der Basalebene),

$$
\begin{aligned}
& u_{1}=\frac{b_{1}}{4 \pi}[A-B] \\
& u_{2}=\frac{b_{1}}{4 \pi}[D-C] .
\end{aligned}
$$

Und die Lösung für den Fall, dass die Stufenkomponente $\left(b_{1}, b_{2}\right)$ parallel zu $x_{2}$ ist $\left(b_{1}=0\right)$, d.h. der Burgersvektor liegt in der prismatischen Ebene, lautet

$$
\begin{aligned}
& u_{1}=\frac{b_{2}}{4 \pi}[C+D] \\
& u_{2}=\frac{b_{2}}{4 \pi}[A+B] .
\end{aligned}
$$

\section{B.2 Stapelfehlerenergie}

Um die Energie der Stapelfehler in aufgespaltenen Versetzungen zu berechnen, werden zunächst die Energiefaktoren $K_{i j}$ benötigt. Aufgrund der Symmetrie (2-zählige Achse entlang Versetzungslinie, 6-zählige Achse entlang $x_{2}$ ) der beiden Fälle, die in dieser Arbeit auftreten, gilt für sie

$$
K_{i j}=\left[\begin{array}{ccc}
K_{1} & 0 & 0 \\
0 & K_{2} & 0 \\
0 & 0 & K_{3}
\end{array}\right]
$$


mit

$$
\begin{aligned}
K_{1} & =\frac{1}{S_{11} \sqrt{2\left(\lambda^{2}-\Lambda\right)}} \\
K_{2} & =\frac{K_{1}}{\lambda^{2}} \\
K_{3} & =\frac{1}{\sqrt{S_{44} S_{55}}} .
\end{aligned}
$$

Die elastische Wechselwirkungsenergie (pro Versetzungslänge) für zwei gerade und parallele Versetzungen mit den Burgersvektoren $b^{(1)}$ und $b^{(2)}$ und dem Abstand $d$ lautet (Abschnitt 3.1.4 in [149])

$$
E_{12}(d)=-\sum_{i, j} \frac{K_{i j} b_{i}^{(1)} b_{j}^{(2)}}{2 \pi} \ln d+\ldots,
$$

die ausgelassenen Terme in obiger Formel hängen nicht vom Abstand $d$ ab.

Die Gesamtenergie der aufgespaltenen Versetzung ist die Summe der Selbstenergien der beiden Partialversetzungen $E_{1}$ und $E_{2}$ (beide unabhängig von $d$ ), ihrer Wechselwirkungsenergie $E_{12}$ sowie der Stapelfehlerenergie $\gamma d$ :

$$
E=E_{1}+E_{2}+E_{12}(d)+\gamma d
$$

Im Gleichgewicht ist die Gesamtenergie minimal, so dass aus $\frac{\partial E}{\partial d}=0$ die Stapelfehlerenergie folgt:

$$
\gamma=\frac{1}{2 \pi d} \sum_{i, j} K_{i j} b_{i}^{(1)} b_{j}^{(2)}
$$




\section{Phase an der Ausscheidungsoberfläche}

An der Oberfläche der $\mathrm{GaMn}_{3} \mathrm{~N}$-Ausscheidungen wurde ein 10-20 nm dünner Film einer weiteren polykristallinen Phase gefunden (s. Abb. C.1), die nicht identifiziert werden konnte.

In Abb. C.2 ist der Betrag der Fouriertransformation einer Objektwellenrekonstruktion eines größeren Kristallits (Probe J0121) zu sehen. Er kann mit einem Beugungsbild verglichen werden, da auch die Intensitäten der Reflexe interpretiert werden können, was bei normalen Diffraktogramme aufgrund der nichtlinearen Bildentstehung nicht möglich ist. Dieses „Beugungsbild“ lässt sich kubisch indizieren (Zonenachse [11̄0]), die Winkel zwischen den $\{111\}$ Reflexen entsprechen wie im kubischen Fall $109^{\circ}$. Die in der $f c c$-Struktur verbotenen Reflexe, wie $\{100\}$ oder $\{110\}$, sind auch hier nicht auffindbar. Auffällig sind die schwachen $\{200\}$ und starken $\{440\}$-Reflexe. Die kubische Gitterkonstante dieser Phase ist $0.84 \pm 0.05 \mathrm{~nm}$.

Eine EDX-Analyse dieser Phase war aufgrund ihres Auftretens am Probenrand nicht möglich, da das Signal der Klebeschicht nicht vom Signal der unbekannten Phase zu trennen war. 


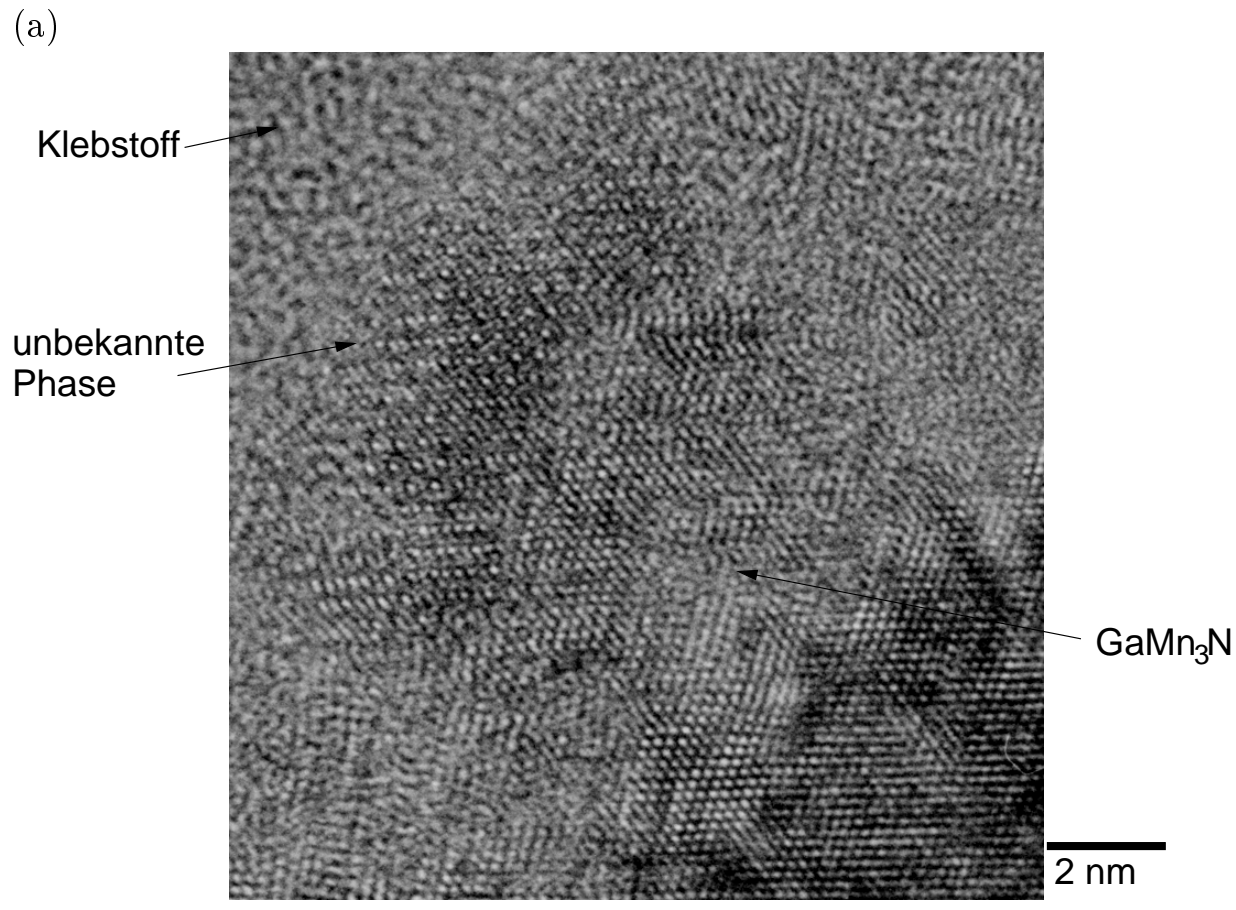

(b)

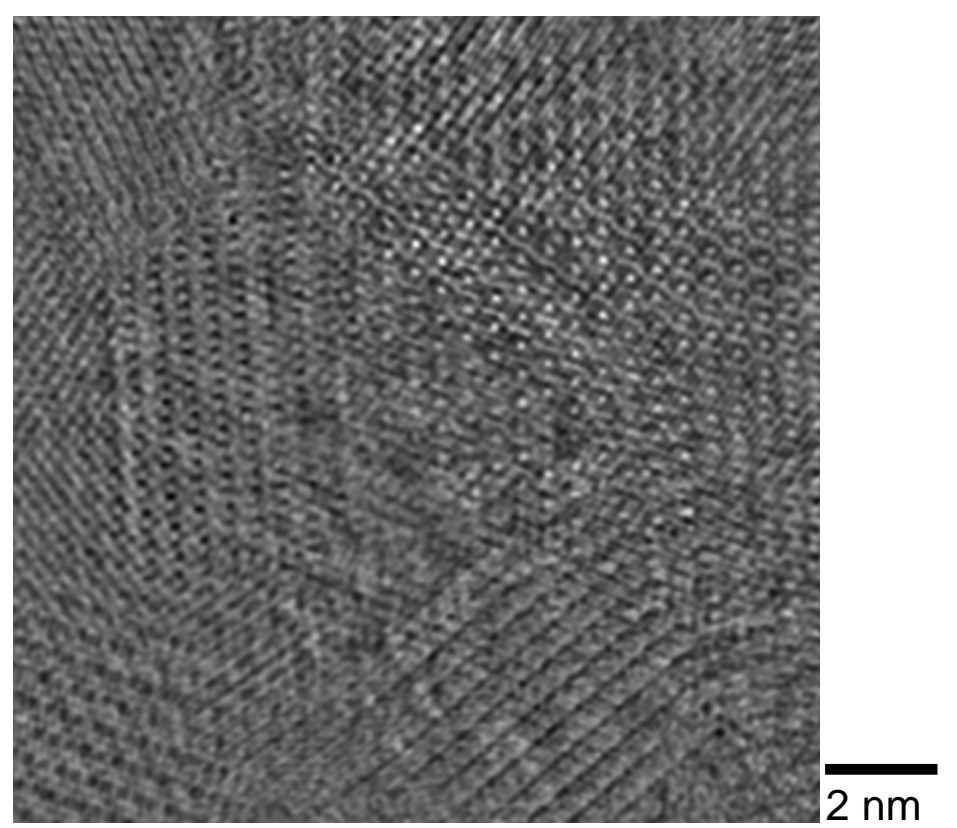

Abbildung C.1: (a) HRTEM-Abbildung der unbekannten Phase in Probe J0087. Der Kristallit in der Mitte der Abbildung zeigt ein fcc-[110]-Zonenachsenmuster. (b) Phasenrekonstruktion von Probe J0121. Hier lassen sich verschiedene Kristallite der unbekannten Phase finden. Der große Kristallit oben rechts zeigt ein ähnliches Muster zu dem Kristallit in (a). 


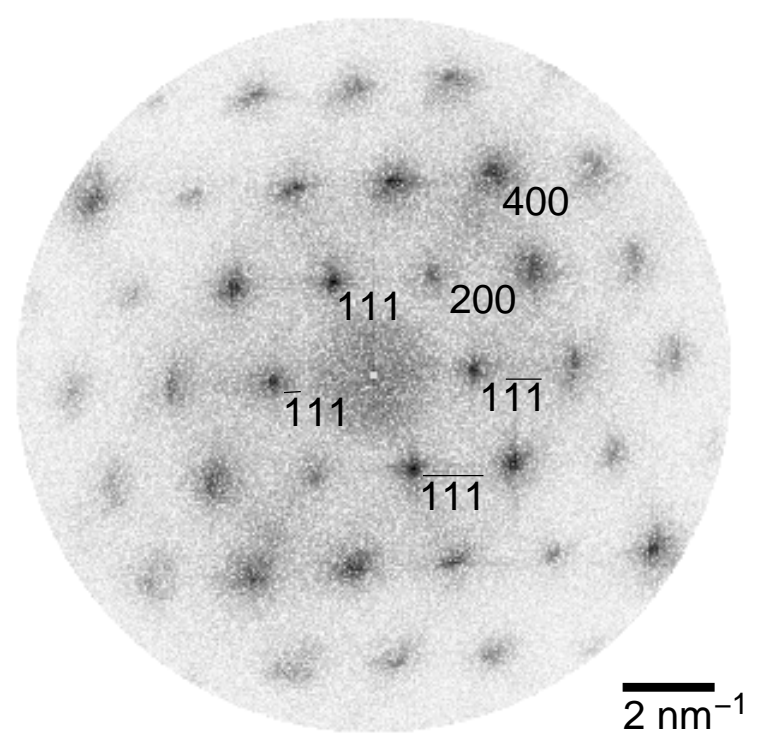

Abbildung C.2: Betrag der Fouriertransformation einer Defokusserienrekonstruktion der unbekannten Phase (logarithmisch skaliert). 


\section{Probenpräparation}

\section{D.1 Querschnittsproben}

Die Querschnittsproben wurden bis auf das Ionenätzen nach dem bei Seibt [150] beschriebenen Verfahren hergestellt.

Die Ionendünnung wurde unter einseitigem Beschuss mit einer Azimuthalwinkeloszillation mit einer Amplitude von $20^{\circ}$ in einer BAL-TEC RES 0201 Ionenätzanlage vorgenommen. Der Beschuss wurde senkrecht zur Klebenaht vorgenommen.

Bei einem Einstrahlwinkel von $17^{\circ}$ wurde zunächst mit $4.5 \mathrm{keV}$-Ar-Ionen die Probe bei einem etwa 20-minütigen Wechsel der Beschussseite gedünnt. Sobald eine Eindellung des Substrats erkennbar war, wurde die Beschleunigungsspannung auf $3 \mathrm{keV}$ heruntergesetzt und der Beschuss mit einem Einstrahlwinkel von 10-12 ${ }^{\circ}$ fortgesetzt, bis das Loch die GaN-Schichten der Querschnittsprobe erreicht hatte. Kurz vor Beendigung des Ionenätzens wurde die Frequenz der Seitenwechsel auf 5-10 Minuten verringert.

\section{D.2 Aufsichtproben}

Die Aufsichtsproben wurden zunächst wie die Querschnittsproben geklebt und gesägt. Das Schleifen und Polieren wurde danach nur einseitig vorgenommen. Aus diesen Querschnittsproben wurde in einer Nova 600 Nanolab-Anlage mittels Focused-Ion-Beam (FIB) eine TEMQuerschnittspräparation durchgeführt [151]. Dabei lag dieser „Querschnitt" der Querschnittsprobe in der Ebene der GaN-Schicht (s. Abb. 5.3).

Der Omniprobe-Probenträger, an dem die TEM-Probe befestigt ist, wurde zunächst auf einen Kupferprobenträger geklebt (s. Abb. D.1). Dieser Probenträger wurde in eine Gatan-DuomillIonenätze eingebaut und unter einem Winkel von $12^{\circ}$ mit $2.5 \mathrm{keV}$ - bis $3 \mathrm{keV}$-Ar-Ionen für 1-2 Minuten von jeder Seite beschossen. Dieser Ionenätzvorgang wurde unter ständigem Kontrollieren der Probe im TEM wiederholt, bis die GaN:Mn-Schicht die notwendige Elektronentransparenz erreicht hatte. 


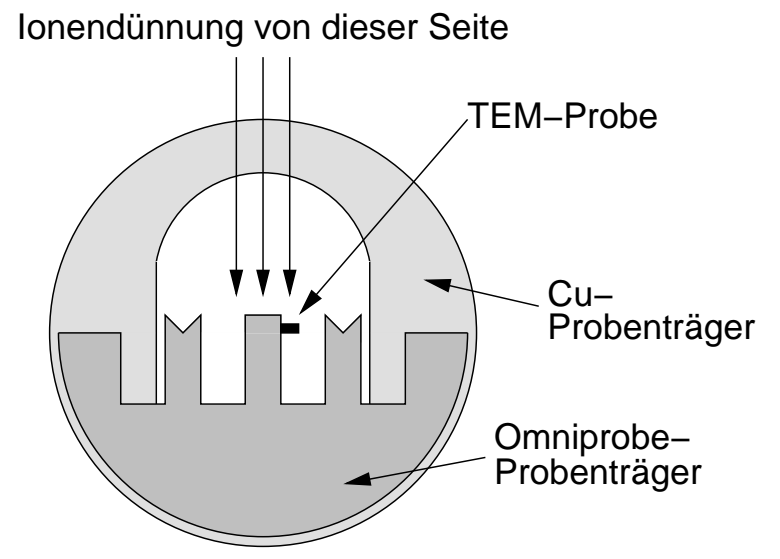

Abbildung D.1: Geometrie beim Ionenätzen der Aufsichtsprobe (nicht maßstabsgerecht). 


\section{E Parameter des Mikroskops}

Sämtliche TEM-Untersuchungen dieser Arbeit wurden an einem Philips CM200-UT FEG Mikroskop durchgeführt. Es ist mit einer Feldemissionskathode ausgerüstet und seine Beschleunigungsspannung beträgt $200 \mathrm{kV}$. Die EDX-Untersuchungen wurden mit einem Si:Li Detektor und dem Link-ISIS System der Firma Oxford durchgeführt. Die verwendeten CCD-Kamera handelt stammt von der Firma Gatan (Modell: 694).

Die weiteren mikroskopspezifischen Parameter lauten:

\begin{tabular}{lc} 
Parameter & \\
\hline \hline Wellenlänge $\lambda$ & $2.508 \mathrm{pm}$ \\
Sphärische Aberration $C_{s}$ & $0.49 \mathrm{~mm}$ \\
Defokusstreuung $\Delta$ & $4.0 \mathrm{~nm}$ \\
Semikonvergenzwinkel der Beleuchtung $\alpha$ & $0.2 \mathrm{mrad}$ \\
\hline
\end{tabular}




\section{Literaturverzeichnis}

[1] S. A. Wolf, D. D. Awschalom, R. A. Buhrman, J. M. Daughton, S. von Molnár, M. L. Roukes, A. Y. Chtchelkanova und D. M. Treger, Spintronics: A Spin-Based Electronics Vision for the Future, Science 294, 1488 (2001).

[2] J. M. Kikkawa und D. D. Awschalom, Resonant Spin Amplification in n-type GaAs, Phys. Rev. Let. 80, 4313 (1998).

[3] A. G. Huibers, M. Switks, C. M. Marcus, K. Campman und A. C. Gossard, Dephasing in Open Quantum Dots, Phys. Rev. Let. 81, 200 (1998).

[4] D. P. DiVincenzo, Quantum Computation, Science 270, 255 (1995).

[5] G. Schmidt, D. Ferrand, L. W. Molenkamp, A. T. Filip und B. J. van Wees, Fundamental obstacle for electrical spin injection from a ferromagnetic metal into a diffusive semiconductor, Phys. Rev. B 62, R4790 (2000).

[6] X. Jiang, R. Wang, R. M. Shelby, R. M. Macfarlane, S. R. Bank, J. S. Harris und S. S. P. Parkin, Highly Spin-Polarized Room-Temperature Tunnel Injector for Semiconductor Spintronics using MgO(100), Phys. Rev. Let. 94, 056601 (2005).

[7] H. Ohno, Making Nonmagnetic Semiconductors Ferromagnetic, Science 281, 951 (1998).

[8] T. Dietl, H. Ohno, F. Matsukura, J. Cibert und D. Ferrand, Zener Model Description of Ferromagnetism in Zinc-Blende Magnetic Semiconductors, Science 287, 1019 (2000).

[9] K. Sato, P. H. Dederichs, K. Araki und H. Katayama-Yoshida, Ab initio materials design and Curie temperature of GaN-based ferromagnetic semiconductors, Phys. Stat. Sol (c) 0, 2855 (2003).

[10] S. J. Pearton, C. R. Abernathy, G. T. Thaler, R. M. Frazier, D. P. Norton, F. Ren, Y. D. Park, J. M. Zavada, I. A. Buyana, W. M. Chen und A. F. Hebard, Wide bandgap GaN-base semiconductors for spintronic, J. Phys: Condens. Matter 16, R209 (2004).

[11] C. Liu, F. Yun und H. Morkoc, Ferromagnetism of ZnO and GaN: A Review, J. Mat. Sci.: Materials in Electronics 16, 555 (2005).

[12] G. Möbus, Structure Determination by Quantitative High-Resolution Electron Microscopy (Q-HRTEM), in High-Resolution Imaging and Spectrometry of Materials, herausgegeben von F. Ernst und M. Rühle, Seite 69, Springer, 2003.

[13] E. R. Weber, J. Krüger und C. Kisielowski, New Materials: Gallium Nitride, in Handbook of Semiconductor Technology Volume 1, herausgegeben von K. Jackson und W. Schröter, Wiley-VCH, 2000. 
[14] H. Schulz und K. H. Thiemann, Crystal structure refinement of AlN and GaN, Solid State Communications 23, 815 (1977).

[15] P. Ruterana und G. Nouet, Atomic Structure of Extended Defects in Wurtzite GaN Epitaxial Layers, Phys. Stat. Sol. (b) 227, 177 (2001).

[16] I. Belabbas, P. Ruterana, J. Chen und G. Nouet, The atomic and electronic structure of dislocations in Ga-based nitride semiconductors, Phil. Mag. 86, 2241 (2006).

[17] J. P. Hirth und J. Lothe, Theory of dislocations, 2nd Ed., Krieger Publishing Company, 1982.

[18] C. Stampfl und C. G. Van de Walle, Energetics and electronic structure of stacking faults in AlN, GaN and InN, Phys. Rev. B 57, R15052 (1998).

[19] A. F. Wright, Basal-plane stacking faults and polymorphism in AlN, GaN, and InN, J. Appl. Phys. 82, 5259 (1997).

[20] K. Suzuki und S. Takebuchi, Electron microscopy of dislocation introduced into GaN by plastic deformation, Phil. Mag. Let. 79, 423 (1999).

[21] D. N. Zakharov, Z. Liliental-Weber, B. Wagner, Z. J. Reitmeier, E. A. Preble und R. F. Davis, Structural TEM study of nonpolar a-plane gallium nitride grown on (112̄0) $4 \mathrm{H}$ SiC by organometallic vapor phase epitaxy, Phys. Rev. B 71, 235334 (2005).

[22] H. Blank, P. Delavignette, R. Gevers und S. Amelinckx, Fault structures in Wurtzite, Phys. Stat. Sol. 7, 747 (1964).

[23] C. M. Drum, Intersecting faults on basal and prismatic planes in aluminium nitride, Phil. Mag. 11, 313 (1965).

[24] J. E. Northrup, Theory of the (1210) prismatic stacking fault in GaN, Apl. Phys. Let. 72, 2316 (1998).

[25] P. Vermaut, G. Nouet und P. Ruterana, Observations of two atomic configurations for the $1 \overline{2} 10$ stacking fault in wurtzite (Ga,Al) nitrides, Apl. Phys. Let. 74, 694 (1999).

[26] F. A. Ponce und D. P. Bour, Nitride-based semiconductors for blue and green lightemitting devices, Nature 386, 351 (1997).

[27] V. Narayanan, K. Lorenz, W. Kim und S. Mahajan, Origins of threading dislocations in GaN epitaxial layers grown on sapphire by metalorganic chemical vapor deposition, Appl. Phys. Let. 78, 1544 (2001).

[28] P. H. Dederichs, Dilute Magnetic Semiconductors, in Lecture Manuscript of the 36th Spring School of the Institute of Solid State Research, Forschungszentrum Jülich, 2005.

[29] K. Sato, W. Schweika, P. H. Dederichs und H. Katayama-Yoshida, Low-temperature ferromagnetism in (Ga,Mn)N: Ab initio calculations, Phys. Rev. B 70, 201202(R) (2004).

[30] M. E. Overberg, C. R. Abernathy, S. J. Pearton, N. A. Theodoropoulou, K. T. McCarthy und A. F. Hebard, Indication of ferromagnetism in molecular-beam-epitaxy-derived $N$ type GaMnN, Appl. Phys. Let. 79, 1312 (2001). 
[31] M. L. Reed, N. A. El-Masry, H. H. Stadelmaier, M. K. Ritums, M. J. Reed, C. A. Parker, J. C. Roberts und S. M. Bedair, Room temperature ferromagnetic properties of (Ga,Mn)N, Appl. Phys. Let. 79, 3473 (2001).

[32] N. Theodoropoulou, A. F. Hebard, M. E. Overberg, C. R. Abernathy, S. J. Pearton, S. N. G. Chu und R. G. Wilson, Magnetic and structural properties of Mn-implanted GaN, Appl. Phys. Let. 78, 3475 (2001).

[33] G. T. Thaler, M. E. Overberg, B. Gila, R. Frazier, C. R. Abernathy, S. J. Pearton, J. S. Lee, S. Y. Lee, Y. D. Park, Z. G. Khim, J. Kim und F. Ren, Magnetic properties of $n$-GaMnN thin films, Appl. Phys. Let. 80, 3964 (2002).

[34] T. Sasaki, S. Sonada, Y. Yamamoto, K. Suga, S. Shimizu, K. Kindo und H. Hori, Magnetic and transport characteristics on high Curie temperature ferromagnet of $\mathrm{Mn}$-doped GaN, J. Appl. Phys. 91, 7911 (2002).

[35] K. H. Kim, K. J. Lee, D. J. Kim, H. J. Kim, Y. E. Ihm, D. Djayaprawira, M. Takahashi, C. S. Kim, C. G. Kim und S. H. Yoo, Magnetotransport of p-type GaMnN assisted by highly conductive precipitates, Appl. Phys. Let. 82, 1775 (2003).

[36] S. Dhar, O. Brandt, A. Trampert, K. J. Friedland, Y. J. Sun und K. H. Ploog, Observation of spin-glass behavior in homogeneous $(G a, M n) N$ layers grown by reactive molecularbeam epitaxy, Phys. Rev. B 67, 165205 (2003).

[37] S. Dhar, O. Brandt, A. Trampert, L. Däweritz, K. J. Friedland, K. H. Ploog, J. Keller, B. Beschoten und G. Güntherodt, Origin of high-temperature ferromagnetism in $(G a, M n) N$ layers grown on $4 \mathrm{H}-\mathrm{SiC}(0001)$ by reactive molecular-beam epitaxy, Appl. Phys. Let. 82, 2077 (2003).

[38] M. Zajac, J. Gosk, M. Kaminska, A. Twardowski, T. Szyszko und S. Podsiadlo, Paramagnetism and antiferromagnetic d-d coupling in GaMnN magnetic semiconductor, Appl. Phys. Let. 79, 2432 (2001).

[39] B. K. Rao und P. Jena, Giant Magnetic Moments of Nitrogen-Doped Mn Clusters and their Relevance to Ferromagnetism in Mn-Doped GaN, Phys. Rev. Let. 89, 185504 (2002).

[40] M. van Schilfgaarde und O. N. Mryasov, Anomalous exchange interactions in III-V dilute magnetic semiconductors, Phys. Rev. B 63, 233205 (2001).

[41] T. Hynninen, H. Raebiger, J. von Boehm und A. Ayuela, High Curie temperatures in (Ga,Mn)N from Mn clustering, Appl. Phys. Let. 88, 122501 (2006).

[42] K. H. Kim, K. J. Lee, D. J. Kim, C. S. Kim, H. C. Lee, C. G. Kim, S. H. Yoo, H. J. Kim und Y. E. Ihm, Improvement of magnetic property of GaMnN by codoping of $M g$, J. Appl. Phys. 93, 6793 (2003).

[43] M. Kocan, J. Malindretos, M. Röver, J. Zenneck, T. Niermann, D. Mai, M. Bertelli, M. Seibt und A. Rizzi, Mn incorporation in GaN thin layers grown by molecular beam epitaxy, Semicond. Sci. Tech. 21, 1348 (2006). 
[44] J. P. Bouchaud, Propriétés Magnétiques et Structurales des Carbonitrures de Manganèse et des Perorowskites $M n_{3} G a C$ et $M n_{3} G a N$, Ann. Chim. 3, 81 (1968).

[45] D. Fruchart und E. F. Bertaut, Magnetic Studies of the Metallic Perovskite-Type Compounds of Manganese, J. Phys. Soc. Jpn. 44, 781 (1978).

[46] P. L'Heritier, D. Fruchart, R. Madar und R. Fruchart, Instabilities Electroniques dans les Composes du Manganese de Type Perovskite Metallique. Relation avec les Changements de Valence dans les Composes de Terres Rares, Mat. Res. Bull. 14, 1089 (1979).

[47] R. Giraud, S. Kuroda, S. Marcet, E. Bellet-Amalric, X. Biquard, B. Barbara, D. Fruchart, D. Ferrand, J. Cibert und H. Mariette, Ferromagnetic $G a_{1-x} M n_{x} N$ epilayers vs. antiferromagnetic $\mathrm{GaMn}_{3} N$ clusters, Europhys. Let. 65, 553 (2004).

[48] K. J. Lee, F. C. Yu, D. J. Kim, B. G. Kim, Y. H. Kang, H. J. Kim und Y. E. Ihm, Behavior of precipitates in GaMnN, Phys. Stat. Sol (b) 241, 1525 (2004).

[49] M. Zajac, J. Gosk, E. Grzanka, M. Kaminska, A. Twardowski, B. Strojek, T. Szyszko und S. Podsiadlo, Possible origin of ferromagnetism in (Ga,Mn)N, J. Appl. Phys. 93, 4715 (2003).

[50] Z. S. Popovic, S. Satpathy und W. C. Mitchel, Electronic structure of the substitutional versus interstitial manganese in GaN, Phys. Rev. B 70, 161308(R) (2004).

[51] K. M. Yu, W. Walukiewicz, T. Wojtowicz, I. Kuryliszyn, X. Liu, Y. Sasaki und J. K. Furdyna, Effect of the location of $M n$ sites in ferromagnetic $G a_{1-x} M n_{x} A s$ on its Curie temperature, Phys. Rev. B 65, 201303(R) (2002).

[52] R. K. Singh, S. Y. Wu, H. X. Liu, L. Gu, D. J. Smith und N. Newman, The role of Cr substitution on the ferromagnetic properties of $G a_{1-x} C r_{x} N$, Appl. Phys. Let. 86, 012504 (2005).

[53] Y. L. Soo, G. Kioseoglou, S. Kim, S. Huang, Y. H. Kao, S. Kuwabara, S. Owa, T. Kondo und H. Munekata, Local Structure and chemical valency of Mn impurities in wideband-gap III-V magnetic alloy semiconductores $G a_{1-x} M n_{x} N$, Appl. Phys. Let. 79, 3926 (2001).

[54] X. Biquard, O. Proux, J. Cibert, D. Ferrand, H. Mariette, R. Giraud und B. Barbara, Local Structure and Valence State of $M n$ in $G a_{1-x} M n_{x} N$ Epilayers, J. Supercond. 16, 127 (2003).

[55] O. Sancho-Juan, A. Cantarero, G. Martínez-Criado, D. Olguín, N. Garro, A. Cros, M. Salomé, J. Susini, S. Dhar und K. Ploog, X-ray absorption near egde spectroscopy at the Mn K-edge in highly homogeneous GaMnN diluted magnetic semiconductors, Phys. Stat. Sol. (b) 243, 1692 (2006).

[56] S. Kuroda, S. Marcet, E. Bellet-Amalric, J. Cibert, H. Mariette, S. Yamamoto, T. Sakai, T. Ohshima und H. Itoh, Structural analysis of $(G a, M n) N$ epilayers and self-organized dots using MeV ion channeling, Phys. Stat. Sol. (a) 203, 1724 (2006).

[57] D. B. Williams und C. B. Carter, Transmission Electron Microscopy, Plenum Press, 1996. 
[58] D. Van Dyck, H. Lichte und K. D. van der Mast, Sub-ångström structure characterisation: the Brite-Euram route towards one ångström, Ultramicroscopy 64, 1 (1996).

[59] P. Kohler-Redlich und J. Mayer, Quantitative Analytical Transmission Electron Microscopy, in High-Resolution Imaging and Spectrometry of Materials, herausgegeben von F. Ernst und M. Rühle, Seite 119, Springer, 2003.

[60] M. Lehmann, Quantitative Electron Holography with Atomic Resolution (Habilitationsschrift), T.U. Dresden, 2004.

[61] P. E. Batson, N. Dellby und O. L. Krivanek, Sub-ångstrom resolution using aberration corrected electron optics, Nature 418, 617 (2002).

[62] K. Xiu und J. M. Gibson, Study of quadrupole-octopole $C_{c}$ corrector for the large gap HREM, Optik 112, 521 (2001).

[63] G. Cliff und G. W. Lorimer, The quantitative analysis of thin specimen, J. Microscopy 103, 203 (1975).

[64] H. Müller und H. Rose, Electron Scattering, in High-Resolution Imaging and Spectrometry of Materials, herausgegeben von F. Ernst und M. Rühle, Seite 9, Springer, 2003.

[65] C. Gerthsen, H. O. Kneser und H. Vogel, Physik 16. Auflage, Springer-Verlag, 1992.

[66] J. M. Cowley, Twenty forms of electron holography, Ultramicroscopy 41, 335 (1992).

[67] D. Typke und K.Dierksen, Determination of image aberrations in high-resolution electron microscopy using diffractogram and cross-correlation methods, Optik 99, 155 (1995).

[68] A. Thust, M. H. F. Overwijk, W. M. J. Coene und M. Lentzen, Numerical correction of lens aberrations in phase-retrieval HRTEM, Ultramicroscopy 64, 249 (1996).

[69] J. C. H. Spence, Experimental High-Resolution Electron Microscopy (2nd Ed.), Oxford University Press, New York, 1988.

[70] O. Scherzer, The Theoretical Resolution Limit of the Electron Microscope, J. Appl. Phys. 20, 20 (1948).

[71] H. Rose, Advances in Electron Optics, in High-Resolution Imaging and Spectrometry of Materials, herausgegeben von F. Ernst und M. Rühle, Seite 189, Springer, 2003.

[72] W. M. J. Coene, A. Thust, M. Op de Beeck und D. Van Dyck, Maximum-likelihood method for focus-variation image reconstruction in high resolution transmission electron microscopy, Ultramicroscopy 64, 109 (1996).

[73] K. Ishizuka, Contrast transfer of crystal images in TEM, Ultramicroscopy 5, 55 (1980).

[74] H. Alexander, Physikalische Grundlagen der Elektronenmikroskopie, Teubner Studienbücher, 1997.

[75] J. M. Zuo, Electron detection characteristics of slow-scan CCD camera, Ultramicroscopy 66, 21 (1996). 
[76] R. R. Meyer, Quantitative Automated Object Wave Restoration in High-Resolution Electron Microscopy, Dissertation, TU Dresden, 2002.

[77] M. Seibt, Private Mitteilungen.

[78] M. Op de Beeck, D. Van Dyck und W. Coene, Wave function reconstruction in HRTEM: the parabola method, Ultramicroscopy 64, 167 (1996).

[79] W. O. Saxton, What is the focus variation method? Is it new? Is it direct?, Ultramicroscopy 55, 171 (1994).

[80] Earl J. Kirkland, Improved high resolution image processing of bright field electron micrographs: I. Theory, Ultramicroscopy 15, 151 (1984).

[81] P. Hirsch, A. Howie, R. B. Nicholson, D. W. Pashley und M. J. Whelan, Electron Microscopy of Thin Crystals; 2nd. revised Ed., Krieger Publishing Company, 1977.

[82] J. C. H. Spence und J. M. Zuo, Electron Microdiffraction, Plenum Press, New York, 1992.

[83] A. Weickenmeier und H. Kohl, Computation of Absorptive Form Factors for High-Energy Electron Diffraction, Acta Cryst. A 47, 590 (1991).

[84] M. J. Hÿtch und W. M. Stobbs, Quantitative comparision of high resolution TEM images with image simulations, Ultramicroscopy 53, 191 (1994).

[85] C. B. Boothroyd, Why don't high-resolution simulations and images match?, J. Microscopy 190, 99 (1998).

[86] C. B. Boothroyd, Quantification of high-resolution electron microscope images of amorphous carbon, Ultramicroscopy 83, 159 (2000).

[87] C. B. Boothroyd, Quantification of lattice images: the contribution from diffuse scattering, J. El. Microscopy 51, S279 (2002).

[88] A. Howie, Hunting the Stobbs factor, Ultramicroscopy 98, 73 (2004).

[89] D. Van Dyck, H. Lichte und J. C. H. Spence, Inelastic scattering and holography, Ultramicroscopy $\mathbf{8 1}, 187$ (2000).

[90] Z. L. Wang, The 'Frozen-Lattice' Approach for Incoherent Phonon Excitation in Electron Scattering. How Accurate Is It?, Acta Cryst. A 54, 460 (1998).

[91] F. Thon, Zur Defokussierungsabhängigkeit des Phasenkontrastes bei der elektronenmikroskopischen Abbildung, Z. Naturforschung 21, 476 (1966).

[92] K. Thiel, Strukturelle Untersuchung der amorph/kristallinen Grenzfläche mittels quantitativer hochauflösender Transmissionselektronenmikroskopie an den Systemen a-Si/c-Si und $a-G e / c-S i$, Dissertation, Universität Göttingen, 2006.

[93] Q. Fu und H. Lichte, Holographic measurement of the wave aberration of an electron microscope by means of the phases in the Fourier spectrum, J. Microscopy 179, 112 (1995). 
[94] R. R. Meyer, A. I. Kirkland und W. O. Saxton, A new method for the determination of the wave aberration function for high resolution TEM. 1. Measurement of the symmetric aberrations, Ultramicroscopy 92, 89 (2002).

[95] A. Steinecker und W. Mader, Measurement of lens aberrations by means of image displacements in beam-tilt series, Ultramicroscopy 81, 149 (2000).

[96] F. Zemlin, K. Weiss, P. Schiske, W. Kunath und K.-H. Herrmann, Coma-free alignment of high resolution electron microscopes with the aid of optical diffractograms, Ultramicroscopy 3, 49 (1978).

[97] R. R. Meyer, A. I. Kirkland und W. O. Saxton, A new method for the determination of the wave aberration function for high-resolution TEM. 2. Measurement of the antisymmetric aberrations, Ultramicroscopy 99, 115 (2004).

[98] A. I. Kirkland und R. R. Meyer, 'Indirect' High Resolution Transmission Electron Microscopy: Aberration Measurement and Wavefunction Reconstruction, Microsc. Microanal. 10, 401 (2004).

[99] A. Thust, W. M. J. Coene, M. Op de Beck und D. Van Dyck, Focal-series reconstruction in HRTEM: simulation studies on non-periodic objects, Ultramicroscopy 64, 211 (1996).

[100] H. Lichte, Optimum focus for taking electron holograms, Ultramicroscopy 38, 13 (1991).

[101] J. Frank, The Role of Correlation Techniques in Computer Image Processing, in Computer Processing of Electron Microscope Images, herausgegeben von P. W. Hawkes, Seite 187, Springer, 1980.

[102] S. Ulhemann und M. Haider, Residual wave aberrations in the first spherical aberration corrected transmission electron microscope, Ultramicroscopy 72, 109 (1998).

[103] G. Chand, W. O. Saxton und A. I. Kirkland, Aberration measurement and automated alignment of the TEM, Inst. Phys. Conf. Ser. 147, 297 (1995).

[104] W. O. Saxton, Aberration measurement and compensation for near-1A microscopy, Inst. Phys. Conf. Ser. 147, 73 (1995).

[105] S. P. Mallick, B. Carragher, C. S. Potter und D. J. Kriegmann, ACE: Automated CTF Estimation, Ultramicroscopy 104, 8 (2005).

[106] Research Systems Inc., IDL Version 5.1 Reference Guide, 1998.

[107] W. H. Press, S. A. Teukolsky, W. T. Vetterling und B. P. Flannery, Numerical Recipes in C. 2nd Ed., Cambridge University Press, 2002.

[108] P. A. Stadelmann, EMS - a software package for electron diffraction analysis and HREM image simulation in materials science, Ultramicroscopy 21, 131 (1987).

[109] H. Müller, H. Rose und P. Schorsch, A coherence function approach to image simulation, J. Microsc. 190, 73 (1998). 
[110] M. J. Hÿtch, Analysis of Variations in Structure from High Resolution Electron Microscope Images by Combining Real Space and Fourier Space Information, Microsc. Microanal. Microstruct. 8, 41 (1997).

[111] M. Hÿtch, E. Snoeck und R. Kilaas, Quantitative measurement of displacement and strain fields from HREM micrographs, Ultramicroscopy 74, 131 (1998).

[112] M. Hÿtch und T. Plamann, Imaging conditions for reliable measurement of displacement and strain in high-resolution electron microscopy, Ultramicroscopy 87, 199 (2001).

[113] H. Seitz, K. Ahlborn, M. Seibt und W. Schröter, Sensitivity limits of strain mapping procedures using high-resolution microscopy, J. Microscopy 190, 184 (1998).

[114] A. T. Blumenau, J. Elsner, R. Jones, M. I. Heggie, S. Öberg, T. Frauenheim und P. R. Briddon, Dislocations in hexagonal and cubic GaN, J. Phys: Cond. Mat. 12, 10223 (2000).

[115] G. Savini, M. I. Heggie, C. P. Ewels, N. Martsinovich, R. Jones und A. T. Blumenau, Structure and Energy of the $90^{\circ}$ Partial Dislocations in Wurtzite-GaN, Mat. Sci. Forum 483-485, 1057 (2005).

[116] J. Kioseoglou, G. P. Dimitrakopulos, P. Komninou und T. Karakostas, Atomic structures and energies of partial dislocations in wurtzite GaN, Phys. Rev. B 70, 035309 (2004).

[117] V. A. Savastenko und A. U. Sheleg, Study of the Elastic Properties of Gallium Nitride, Phys. Stat. Sol. (a) 48, K135 (1978).

[118] A. F. Wright, Elastic Properties of zinc-blende and wurtzite AlN, GaN, and InN, J. Appl. Phys. 82, 2833 (1997).

[119] M. Yamaguchi, T. Yagi, T. Azuhata, T. Sota, K. Suzuki, S. Chichibu und S. Nakamura, Brillouin scattering study of gallium nitride: elastic stiffness constants, J. Phys: Condens. Matter 9, 241 (1997).

[120] Y. Takagi, M. Ahart, T. Azuhata, T. Sota, K. Suzuki und S. Nakamura, Brillouin scattering study in the GaN epitaxial layer, Physica B 219\&220, 547 (1996).

[121] R. B. Schwarz, K. Khachaturyan und E. R. Weber, Elastic moduli of gallium nitride, Appl. Phys. Let. 70, 1122 (1997).

[122] A. Polian, M. Grimsditch und I. Grzegory, Elastic constants of gallium nitride, J. Appl. Phys. 79, 3343 (1996).

[123] P. Komninou, J. Kioseoglou, G. P. Dimitrakopulos, T. Kehagias und T. Karakostas, Partial dislocations in wurtzite GaN, Phys. Stat. Sol. (a) 202, 2888 (2005).

[124] P. Villars und L. D. Calvert, Pearson's Handbook of Crystallographic Data for Intermetallic Phases (2nd Ed.), ASM International, 1991.

[125] X. M. Cai, A. B. Djurisic, M. H. Xie, H. Liu, X. X. Zhang, J. J. Zhu und H. Yang, Ferromagnetism in Mn and Cr doped GaN by thermal diffusion, Mat. Sci. and Eng. B 117, 292 (2005). 
[126] T. Zywietz, J. Neugebauer und M. Scheffler, Adatom diffusion at GaN (0001) and (0001) surfaces, Appl. Phys. Let. 73, 487 (1998).

[127] E. Calleja, M. A. Sánchez-García, F. Calle, F. B. Naranjo, E. Munoz, U. Jahn, K. Ploog, J. M. Calleja, K. Saarinen und P. Hautojärvi, Molecular beam epitaxy growth and doping of III-nitrides on Si(111): layer morphology and doping efficiency, Mat. Sci. and Eng. B 82, 2 (2001).

[128] J. C. H. Spence und J. Taftø, ALCHEMI: a new technique for locating atoms in small crystals, Journal of Microscopy 130, 147 (1983).

[129] L. J. Allen und T. W. Joseffson, Inelastic scattering of fast electrons by crystals, Phys. Rev. B 52, 3184 (1995).

[130] I. P. Jones, Determining the Locations of Chemical Species in Ordered Compounds: ALCHEMI, Advances in Imaging and Electron Physics 125, 63 (2002).

[131] C. J. Rossouw und P. R. Miller, Analysis of incoherent channeling patterns formed by $X$-ray emission from host lattice species and interstitial $\mathrm{Cr}$ in mullite, J. El. Microscopy 48, 849 (1999).

[132] U. Kaiser, Nanocrystal formation in hexagonal SiC after Ge ion implantation, J. of Electron Microscopy 50, 251 (2001).

[133] C. J. Rossouw, C. J. Bettles, T. J. Davis, C. T. Forwood, P. R. Miller und K. Venkatesan, Location of $Z n$ within the $M g_{12}\left(L a_{x} C e_{1-x}\right)$ lattice by X-ray incoherent channeling patterns, Acta Cryst. A 57, 321 (2001).

[134] T. Morimura und M. Hasaka, ALCHEMI for coexistent Heusler and half-Heusler phases in $\mathrm{TiNi}_{1.5} \mathrm{Sn}$, Ultramicroscopy 106, 553 (2006).

[135] L. J. Allen und C. J. Rossouw, Delocalization in electron-impact ionization in a crystalline environment, Phys. Rev. B 47, 2446 (1993).

[136] L. J. Allen, T. W. Josefsson und C. J. Rossouw, Interaction delocalization in characteristic X-ray emission from light elements, Ultramicroscopy 55, 258 (1994).

[137] M. P. Oxley, L. J. Allen und C. J. Rossouw, Correction terms and approximations for atom location by channelling enhanced microanalysis, Ultramicroscopy 80, 109 (1999).

[138] L. J. Allen und T. Joseffson, Validity of generalized scattering equations and corresponding inelastic-cross-section expressions for comprehensive electron diffraction conditions, Phys. Rev. B 53, 11285 (1996).

[139] M. P. Oxley und L. J. Allen, Atomic scattering factors for K-shell and L-shell ionization by fast electrons, Acta Cryst. A 56, 470 (2000).

[140] M. Kocan, AlGaN/GaN MBE 2DEG Heterostructures, Dissertation, RWTH Aachen, 2003.

[141] J. Zenneck, T. Niermann, M. Röver, M. Kocan, J. Malindretos, M. Seibt, R. G. Ulbrich und A. Rizzi, Intra-atomic photoluminescence at $1.42 \mathrm{eV}$ of substituional Mn in GaMnN, submitted to Appl. Phys. Let. (2006). 
[142] K. Tillmann, Private Mitteilungen, 2005.

[143] D. Van Dyck und M. Op de Beeck, A simple intuitive theory for electron diffraction, Ultramicroscopy 64, 99 (1996).

[144] C. T. Koch und S. H. Garofalini, Determining the radial pair-distribution function within intergranular amorphous films by numerical nanodiffraction, Ultramicroscopy 106, 383 (2006).

[145] T. Dietl, Self-organized growth controlled by charge states of magnetic impurities, Nature materials 5, 673 (2006).

[146] K. Sato, H. Katayama-Yoshida und P. H. Dederichs, High Curie Temperature and NanoScale Spinodal Decomposition Phase in Dilute Magnetic Semiconductors, Jap. J. Appl. Phys. 44, L948 (2005).

[147] C. Kisielowski, Strain in GaN Thin Films and Heterostructures, in Gallium Nitride (GaN) II, herausgegeben von J. I. Pankove und T. D. Moustakas, Seite 275, Academic Press, 1999.

[148] M. J. Reed, F. E. Arkun, E. A. Berkman, N. A. Elmasry, J. Zavada, M. O. Luen, M. L. Reed und S. M. Bedair, Effect of doping on the magnetic properties of GaMnN: Fermi level engineering, Appl. Phys. Let. 86, 102504 (2005).

[149] J. W. Steeds, Introduction to Anisotropic Elasticity Theory of Dislocations, Clarendon Press, 1973.

[150] M. Seibt, Elektronenmikroskopische Untersuchungen des Ausscheidungsverhaltens von Nickel in Silizium, Dissertation, Universität Göttingen, 1986.

[151] L. A. Giannuzzi und F. A. Stevie, editors, Introduction to Focused Ion Beams, Springer, 2005. 


\section{Danksagung}

Meinem Doktorvater PD Dr. Michael Seibt danke ich für die Möglichkeit zur Durchführung dieser Doktorarbeit. Ich danke ihm für die stete Diskussionsbereitschaft sowie für die vielen wertvollen Ratschläge und die hilfreiche Kritik im Verlauf dieser Arbeit. Ebenso danke ich ihm für die Unterstützung bei der Elektronenmikroskopie.

Prof. Dr. Reiner Kirchheim danke ich für die bereitwillige Übernahme des Korreferats.

Bei Prof. Dr. Angela Rizzi, Jörg Malindretos, Jan Zenneck, Martin Kocan, Martin Röver, Dong-Du Mai und Marco Bertelli bedanke ich mich für die Herstellung der GaN-Proben sowie für die Diskussionen über das Materialsystem GaN:Mn.

Manfred Schrader danke ich für die Hilfe bei technischen Problemen am TEM und an den Ionenätzen.

Bei Matthias Hahn bedanke ich mich für die FIB-Präparation der Aufsichtsprobe.

Für die angenehme Arbeitsatmosphäre und die ständige Hilfsbereitschaft während meiner Doktorarbeit danke ich insbesondere meinem langjährigen Zimmergenossen Karsten Thiel sowie den anderen Mitgliedern meiner Arbeitsgruppe: Michael Seibt, Wolfgang Schröter, Andreas Sattler, Carsten Rudolf, Hendrik Bergmann, Henning Schuhmann, Philipp Saring, Oliver Voß, Rheda Khalil und Yue-Lin Huang.

Für die Durchsicht meiner Doktorarbeit danke ich Carsten Rudolf, Jörg Malindretos, Karsten Thiel und Oliver Voss. Insbesondere danke ich Julia Wandt und Lars Korte für die Korrektur der vielen sprachlichen Fehler im Manuskript.

Für die angenehme Zusammenarbeit danke ich allen Mitgliedern des IV. Physikalischen Institutes.

Mein tiefster Dank geht an meine Eltern und an meine Großeltern für ihre Unterstützung während meines gesamten Studiums. 



\title{
Lebenslauf
}

\author{
Name: $\quad$ Tore Niermann \\ Geburtstag: $\quad$ 13.05.1976 \\ Geburtsort: Georgsmarienhütte \\ Staatsangehörigkeit: Deutsch
}

\section{Werdegang}

1982-1986 Grundschule Sutthausen, Osnabrück

1986-1988 Orientierungsstufe Kalkhügel, Osnabrück

1988-1995 Graf-Staufenberg-Gymnasium, Osnabrück

$1995 \quad$ Abitur

1995-1996 Zivildienst in der Diakonie-Sozialstation Osnabrück

1996-2001 Physik-Studium an der Georg-August-Universität Göttingen

$2001 \quad$ Diplom

Titel der Diplomarbeit: „Mustererkennungsalgorithmen zur Analyse hochaufgelöster elektronenmikroskopischer Abbildungen komplexer Materialien"

seit 2002 Doktorand unter der Betreuung von PD Dr. M. Seibt am IV.

Physikalischen Institut der Universität Göttingen

2002-2004 Wissenschaftlicher Angestellter am Sonderforschungsbereich 602 der Universität Göttingen

seit 2005 Wissenschaftlicher Angestellter am IV. Physikalischen Institut der Universität Göttingen 